\title{
Time series analysis under model uncertainty
}

Citation for published version (APA):

Lohmeyer, J. H. (2019). Time series analysis under model uncertainty. [Doctoral Thesis, Maastricht University]. ProefschriftMaken Maastricht. https://doi.org/10.26481/dis.20190524jl

Document status and date:

Published: 01/01/2019

DOI:

10.26481/dis.20190524jl

Document Version:

Publisher's PDF, also known as Version of record

\section{Please check the document version of this publication:}

- A submitted manuscript is the version of the article upon submission and before peer-review. There can be important differences between the submitted version and the official published version of record.

People interested in the research are advised to contact the author for the final version of the publication, or visit the DOI to the publisher's website.

- The final author version and the galley proof are versions of the publication after peer review.

- The final published version features the final layout of the paper including the volume, issue and page numbers.

Link to publication

\footnotetext{
General rights rights.

- You may freely distribute the URL identifying the publication in the public portal. please follow below link for the End User Agreement:

www.umlib.nl/taverne-license

Take down policy

If you believe that this document breaches copyright please contact us at:

repository@maastrichtuniversity.nl

providing details and we will investigate your claim.
}

Copyright and moral rights for the publications made accessible in the public portal are retained by the authors and/or other copyright owners and it is a condition of accessing publications that users recognise and abide by the legal requirements associated with these

- Users may download and print one copy of any publication from the public portal for the purpose of private study or research.

- You may not further distribute the material or use it for any profit-making activity or commercial gain

If the publication is distributed under the terms of Article $25 \mathrm{fa}$ of the Dutch Copyright Act, indicated by the "Taverne" license above, 


\section{Time Series Analysis \\ under Model Uncertainty}

Jan Lohmeyer

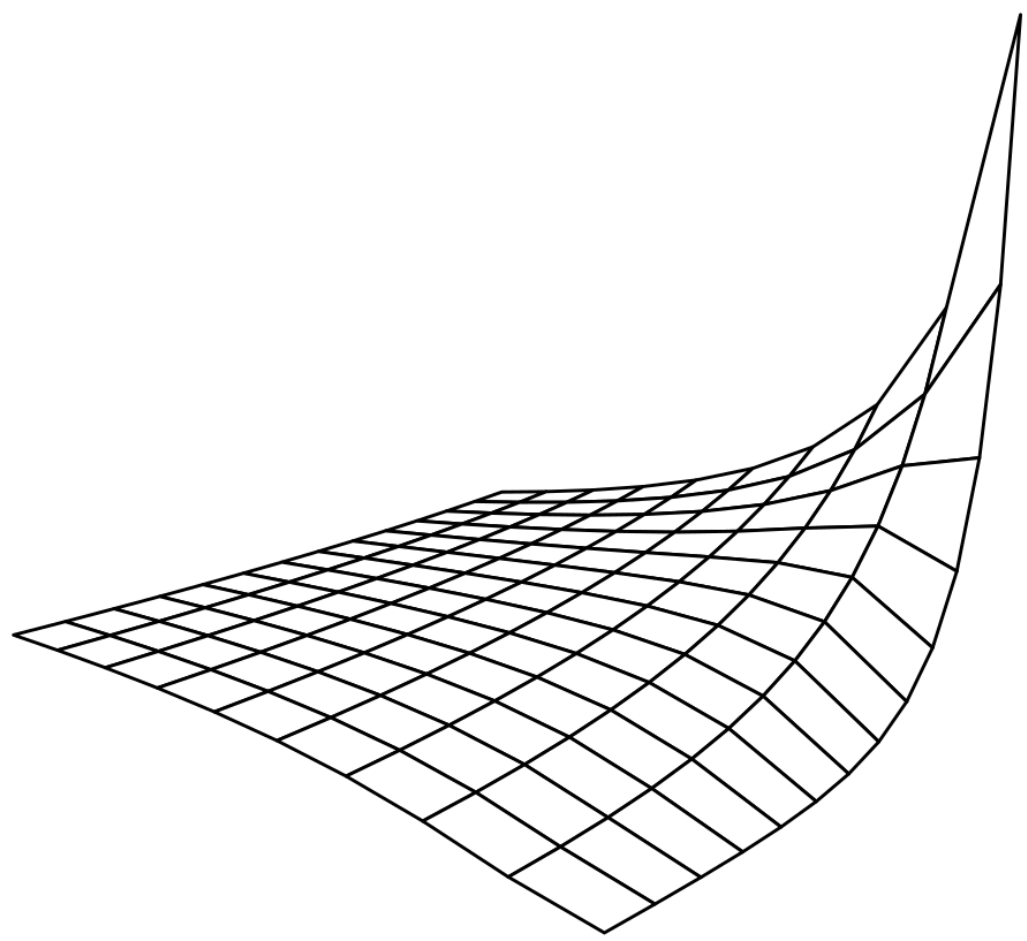




\section{Time Series Analysis under Model Uncertainty}

Jan Lohmeyer 
(C) Jan Lohmeyer, Düsseldorf 2019

All rights reserved. No part of this publication may be reproduced, stored in a retrieval system, or transmitted in any form, or by any means, electronic, mechanical, photocopying, recording or otherwise, without the prior permission in writing from the author.

The book was typeset by the author using $\mathrm{LT}_{\mathrm{E}} \mathrm{X}$.

Published by ProefschriftMaken - www.proefschriftmaken.nl ISBN: 978-94-6380-296-3

Cover illustration: Smoothed averaging weights calculated as $\exp (-i /(2 \kappa)) / \sum_{i} \exp (-i /(2 \kappa)) . \quad \kappa \in\{1, \ldots, 10\}$ increases along the left axis towards the far end, the selection score $i \in\{1, \ldots, 14\}$ decreases on the right axis towards the far end. So lower scores correspond to higher weights, and a smaller value of $\kappa$ to more concentrated weights. 


\section{Time Series Analysis under Model Uncertainty}

\section{DISSERTATION}

to obtain the degree of Doctor at Maastricht University, on the authority of the Rector Magnificus

Prof. dr. Rianne M. Letschert

in accordance with the decision of the Board of Deans, to be defended in public

on Friday 24 May 2019 at 12:00 hours

by

Jan Hendrik Lohmeyer 


\section{Supervisors}

Prof. dr. Franz Palm

Prof. dr. Jean-Pierre Urbain $†$

Prof. dr. Alain Hecq

\section{Assessment Committee}

Prof. dr. Peter Schotman (chair)

Dr. Jan Jacobs, University of Groningen

Prof. dr. Rolf Tschernig, University of Regensburg

Dr. Ines Wilms

This research was financially suppported by the Netherlands Organisation for Scientific Research (NWO). 


\section{Acknowledgements}

I would first like to thank Franz, Jean-Pierre, and Peter who decided to take me on as a $\mathrm{PhD}$ student in their project group and Antoon, who, despite the fact that he got to know me a little at a joint lunch on Tongersestraat, did not veto their decision. We even had a funny misunderstanding about the beginning of my contract, but they never let that become a problem. On the same token I want to thank the NWO for financing my position. It had been my ambition to do a $\mathrm{PhD}$ for several years, and being given this chance was an unexpected case of good luck for me. I thank Heino for supervising my Master thesis. The infrastructure of the SBE, the short routes within the picturesque city, UM Sports, and the open, witty $\mathrm{PhD}$ colleagues of not only the fourth floor made my almost four years in Maastricht a great pleasure.

My greatest thanks go to Franz and Jean-Pierre for being patient, trusting supervisors. They gave me every freedom I could have hoped for and even though being engulfed in various administrative obligations they always made our regular meetings a top priority. It took me a good while, basically until the end of my stay in Maastricht, to understand that they had left the main responsibility for the dissertation with me. I had taken too little initiative, done too little project management for this long-term research project to finish in time. Thank you, Franz for seeing my dissertation through to the end and your positivity. Tragically Jean-Pierre passed away at the end of my employment in Maastricht. He was not only trusting in my research work but also in teaching and kept giving me second chances to improve. He was a straightforward, honest and caring person, and I would hope to be able to carry some of his wisdom and compassion further in my life.

I want to thank my second promoter and the members of the assessment committee for the time and effort they spent on reviewing my thesis and the helpful comments: Thank you Alain Hecq, Peter Schotman, Jan Jacobs, Rolf Tschernig and Ines Wilms. I am also thankful to Alain and Peter for the help they provided during the course of my research.

Thank you office mates Dennis, Martijn, Hanno, and Shashwat for granting me the sunny spot and for much more. Hanno, thank you in particular for the collaboration on our paper. Thank you semi office mates, residents of the fourth floor and beyond, Tim, Andrey, Anne, Oksana, Anne, Rasmus, Simon, Marc, Kouma, Ahmad, Sean, Marina, Alex, Veerle, Andrej, Yuliya, Thomas, Verena, Aida, Ruben, Greg, Etienne, Daria, Henrik, Florian, Alessandro. Thank you 


\section{ACKNOWLEDGEMENTS}

Vincent and Marc for being good listeners and pleasant to be around. Angie, thank you for our smoking conversations. Anna, I would like to do my next PhD with you in the field of irony. I admire you for being able to say anything to anyone and get away with it. Artem, thank you for the Basketball play you drew for me on the white board. It really improved my game! Thank you Rogier, not only for being the Ox guy. Thank you Dominik and Tom for being my sanctuary.

I thank the staff of the QE and Economics departments for always being accessible and helpful, especially Yolanda, Karin, Alex, Michael, Hans, Rudolf, Eric, Stephan, and Lenard. I thank my German girls Martina and Laura for their optimism, and Mira and Annika for embellishing Dutch classes. Thank you Olga and Anastasia for your friendship. Thank you Caro, Judith and Iris for having supported my work. I thank my gymnastics mates Maciek, Lukas, Vincent, Christopher and Jonas, my Kimbria team and the UM Basketball dudes for challenging trainings, Floor for the bootcamp sessions and bike rides, and Fabio, Vincent, Simon and Martin for swimming and cycling fun. Thank you Ben, Sonja and Sylvia for welcoming me in Düsseldorf. Thank you Brayn for supporting and Tatjana, Alex, and Max for showing interest in my research.

I want to thank my family for being just that, my family. Thank you Julia, Joscha, Peer, Michel, Tatjana, Kim, Julian, Felix. My parents Annemarie and Heinrich always supported me in choosing my own paths, and walked them with me, quite literally even, considering the number of relocations they helped me with. 


\section{Contents}

Acknowledgements $\quad$ v

1 Introduction 1

2 A Focused Information Criterion for Locally Misspecified VARs 7

2.1 Introduction . . . . . . . . . . . . . . . . . . 7

2.2 Theory . . . . . . . . . . . . . . . . . . . . . . 9

2.2.1 The Model Framework . . . . . . . . . . . . . . 9

2.2.2 Parameter Estimation and Asymptotics . . . . . . . . . . 9

2.2.3 The Quantities of Interest . . . . . . . . . . . . . 13

2.2.4 The Effects of Inconsistently Estimating Delta . . . . . . . 18

2.3 Simulations ...................... . . . . 20

2.3.1 The Simplified DGP . . . . . . . . . . . . . 22

2.3.2 Simulation Results for an Autoregressive Model . . . . . . . 25

2.3.3 Simulation Results for a Vector Autoregressive Model . . . 26

2.4 Conclusions . . . . . . . . . . . . . . . . . . . . . 31

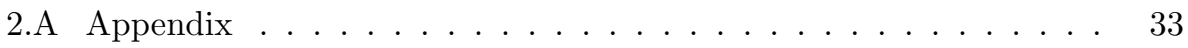

2.A.1 No Gradient Dependence in Simplified Model . . . . . . . . 34

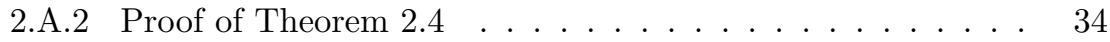

2.A.3 Other Proofs . . . . . . . . . . . . . . 38

3 Consistency of Averaged Impulse Response Estimators in VARs 43

3.1 Introduction . . . . . . . . . . . . . . . . . 43

3.2 Model Setting . . . . . . . . . . . . . . . . . . . . 46

3.3 Consistency of the Averaged Estimator . . . . . . . . . . . . . . . 48

3.3.1 Weight Scaling . . . . . . . . . . . . . . . 49

3.4 Bootstrapping . . . . . . . . . . . . . . . . . . . 50

3.5 Simulation Study . . . . . . . . . . . . . . . . . . . . 52

3.5.1 Simulation Setting of Kilian (2001) . . . . . . . . . . 53

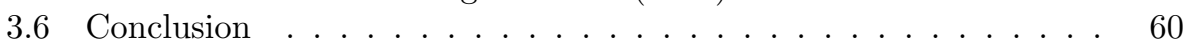

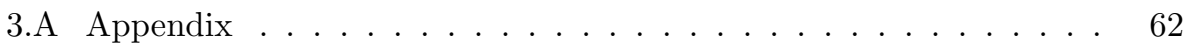

3.A.1 Proof of Lemma $3.1 \ldots \ldots$. . . . . . . . . . . 62

3.A.2 Proof of Theorem $3.2 \ldots \ldots \ldots 6$

3.A.3 Derivation of a Bound for $\kappa \ldots \ldots$. . . . . . . 66

3.A.4 Relation of sAIC and sBIC Weights . . . . . . . . . 66 
3.A.5 Asymptotic Validity of the Bootstrap Procedure . . . . . . 67

3.A.6 Additional Simulation Results . . . . . . . . . . . . . 70

3.A.7 Simulation Setting Imitating Stock and Watson (2001) Data 70

3.A.8 Simulation Setting Based on Lütkepohl et al. (2015) . . . . 75

4 An Application of Model Averaging in Structural VARs $\quad 80$

4.1 Introduction . . . . . . . . . . . . . . . . . . 80

4.2 Estimation Methods . . . . . . . . . . . . . . . . 83

4.3 Estimates . . . . . . . . . . . . . . . . . 87

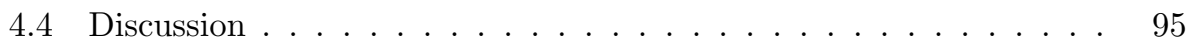

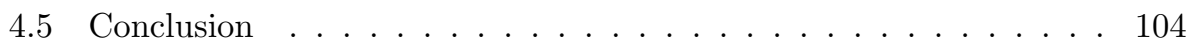

4.A Appendix ........................... 107

4.A.1 Additional Results for Christiano et al. (1999) . . . . . . . 107

4.A.2 Additional Results for Stock and Watson (2001) . . . . . . 108

5 Conclusion $\quad 113$

$\begin{array}{ll}\text { Bibliography } & 115\end{array}$

$\begin{array}{lr}\text { Summary } & 120\end{array}$

$\begin{array}{ll}\text { Valorization Addendum } & 122\end{array}$

$\begin{array}{ll}\text { Curriculum Vitae } & 127\end{array}$ 


\section{Chapter 1}

\section{Introduction}

"The biggest problem we face in econometrics is the uncertainty about the correct specification of our models." Davidson (2004)

The goal of econometrics is to describe a complex reality with a simple model. The first step in an econometric analysis is to select a class of models that seems best fitting for the particular data set at hand. For time series data a different class of models would be considered than for cross sectional data. Should the model be linear or non linear, a state space model, should the variance be modelled separately? Before even starting with her analysis a researcher usually decides on all of these points a priori or with some pretesting of the data. Then, one common approach to end this first part of reducing the universe of models, that are deemed relevant, is to define a class of models that only differs by one type of parameter. Take, for example, the lag order in an autoregressive model. The most fitting parametrization would then be decided on by one of a number of available model selection criteria. The underlying idea of which is in essence to find the "true" model specification within the considered class. But Hansen (2005) pointed out that this idea might be misguided. It is likely that there is not one true underlying model that captures all the relevant explanatory variables. In this thesis we consider two different approaches to a more general idea of finding useful models, focused selection and model averaging.

Focused Selection Focused selection, as introduced by Claeskens and Hjort (2003), puts the interest of the analysis in the foreground. Sometimes the researcher is not interested in the basic parameters of the data generating process, but only in a selection of these or even in a function of these. For example, in an autoregressive analysis one might be interested only in the first autoregressive parameter, or the impulse response at a specific horizon, which is a non linear function of the model parameters. And even if there was one true underlying model for the observed data, the assumed functional relationship between the model's parameters and the actual parameter of interest might be wrong. This is why in focused model selection not the model that best fits the observed data, but the 


\section{CHAPTER 1}

model which is deemed to best estimate the parameter of interest is chosen. On a technical side, the theory on focused parameters is based on the error of the estimated parameter as measured by the asymptotic mean squared error, MSE. Since models that do not include the regressors of the true DGP have a bias, and this bias would diverge in an asymptotic framework, the theory around the focused information criterion, FIC, uses a locally misspecified setting in which all models under consideration include at least the parameters up to the true order, and some auxiliary parameters that shrink to zero asymptotically. It turns out that in practice the division between these two different sets of model parameters is actually of no interest. This is shown in Remark 2.16. Chapter 2 extends the theory of the FIC to stationary multivariate time series models.

In the locally misspecified setting of Chapter 2 the auxiliary parameters shrink to zero at the rate $T^{-1 / 2}$ with $T$ denoting the number of observations. This setting is fundamentally different from a setting where all parameters stay constant, because the data generating process (DGP) is different for different $T$. Similar sequences of so called local alternatives are considered for local power analysis of hypothesis tests, cf. McManus (1991). In their rejoinder to Hjort and Claeskens (2003a) Hjort and Claeskens (2003b) discuss the advantages of and the underlying ideas for considering locally misspecified models, and a series of papers by Leeb and Pötscher (2005) use such models to challenge some common ideas about post-model-selection estimators. In Chapter 3 we assume a DGP with coefficients that are independent of $T$, and so one may wonder whether one of those different settings is more appropriate than the other, or the asymptotic theory for which of these is more helpful. The answer is that the asymptotic results presented in the econometrics literature are derived in the expectation that they may offer a good approximation to the finite-sample behavior of certain estimators. Leeb and Pötscher (2005) make this point very clear in the context of classifying model selection procedures as being consistent or conservative in their Section 2. This is why, in the following chapters, methods based on shrinking as well as constant coefficients are used alongside each other, and why simulations are included in Chapters 2 and 3 to illustrate the finite-sample properties of the introduced estimators.

(Model) Averaging When one acknowledges the idea that there cannot be one true statistical model, model averaging emerges as a natural extension of model selection. Instead of settling to use one particular model for estimation, weights are assigned to the different models under consideration and an averaged estimate of some type is used. A weight of 1 for one model and 0 for all the others leads to a model selection estimator again. One of the earliest references on frequentist model averaging is the paper by Bates and Granger (1969). When using averaging estimators in the following, we remain within one class of models, so we will only average over autoregressive models with different lag orders. This also includes, as is common in time series econometrics, that we only consider nested models. But one might also want to average over different classes of models. This case is treated in Zhang et al. (2013), for example, who allow non-nested autoregressive specifications to be included in the set of models to be averaged, and in forecast combination, where it is not restricted how different forecasters arrive at their 
forecasts, cf. de Pooter et al. (2007). We want to mention that in model averaging there is still a selection step, namely that of deciding which models to consider, but this is not different to carrying out model selection where, as described above, a certain set of models is singled out for closer scrutiny, for which then model scores, e.g. the AIC score, are being calculated. Consider for example a sequence of general to specific tests on the lag order of an autoregressive model. The sequence requires a starting point of some a priori chosen lag order. There are also mixtures of selection and averaging methods, like the adaptive regression by mixing (ARM) method applied in Yuan and Yang (2005). In the ARM, AIC and BIC scores are computed for a large set of models and only a certain prespecified number of the lowest ranked models are kept for the subsequent combination step. In a Bayesian framework model averaging is a well established concept. We do not treat Bayesian model averaging in this book, however, but instead focus on frequentist methods, just like the papers that generated the ideas to our research do, e.g. Hansen (2007), Kilian (1998a).

There are (at least) two types of objects one can average over. The smoothed model averaging estimators of type smoothed AIC (sAIC) combine the estimates of the parameter of interest/focus parameter from the various models. In our applications the parameter of interest is a particular impulse response coefficient (and in the FIC setting also allowed to be a set of those). So when we apply smoothed estimators, the estimates of the focus parameter given by the different models are multiplied with the weights assigned to the respective model and added up. The smoothed AIC (sAIC) and smoothed BIC (sBIC) estimators are used throughout this thesis, and their theoretical properties are discussed in Chapter 3. For this type of combination we suggest the term parameter averaging or estimate averaging.

Another set of objects one might want to average over is that of the estimated parameters of the various models. This is the averaging method used by e.g. Hansen (2007) for Mallows model averaging, where it is shown that weight selection by minimizing Mallow's criterion will asymptotically lead to the lowest possible squared error within a class of estimators. With this averaging method a new model is created that consists of the other models' combined parameters, where the weight that had been assigned to each model is the weight that the parameter receives in the combined model. The model average estimate of the parameter of interest is then the parameter of interest from the combined model. The jackknife model averaging estimator is calculated with this combination scheme. It is applied to stationary autoregressions of infinite order in Zhang et al. (2013), and will be used throughout this thesis. We will use the term model averaging to refer to any of those two combination methods, as this seems to be the term used in the literature so far, but to be more specific the term model averaging or estimator averaging should be reserved to this second type of combination. For our applications, more often than not, the estimates were not very sensitive to the type of averaging. So, combining the underlying models' parameters with sAIC weights instead of combining the estimates of the focus parameters lead to similar estimates. We do not report these results but instead leave this statement to be thoroughly tested in future research. Chapter 3 is dedicated to the smoothed model averaging versions 


\section{CHAPTER 1}

of AIC and BIC model selection. There we show that their asymptotic properties are similar to those of their selection counterparts.

Impulse Response Analysis in Structural VARs The central application of this thesis are impulse response functions. A comprehensive discussion on impulse response analysis can be found in Section 3.7 of Lütkepohl (2005). In a dynamic system impulse responses describe the effect of an exogenous shock. In a multivariate system these effects are especially interesting for macroeconomists, who want to analyze policy implications, as they illustrate the relationships between the different variables, cf. Christiano et al. (1999). The effects can be graphed against time. In a stable or stationary system any shock effect will vanish in the long run. We will refer to these time profiles as impulse response functions and typically call the effect after a certain number of periods an impulse response.

Impulse response analysis on economic data is conducted using structural VAR (sVAR) models. Surveys on sVARs have been authored by Kilian (2013) and Kilian and Lütkepohl (2017). Structural VAR models first appeared in econometrics in the 1980s "as an alternative to traditional large-scale dynamic simultaneous equation models," cf. Kilian (2013). There are two different important representations of a VAR model, the structural and the reduced-form representation. In the former the innovations are uncorrelated, so their covariance matrix is a diagonal matrix, and no contemporaneous effects between the variables exist. This representation allows an economic interpretation, e.g. the calculation of impulse response functions. The underlying assumption is that the uncorrelated structural innovations drive the system's variables. But because not all of the parameters in the structural VAR are identified, estimation would not yield consistent estimates. This is why for estimation the reduced-form representation is used. By reparametrizing the model one representation can be obtained from the other. This reparametrization has to rely on identifying assumptions stemming from, e.g., economic theory, and the different ways of going about this are even to date still a topic of research. The three most commonly used types of using identifying assumptions are to impose restrictions on the short-run, the long-run, or the (algebraic) sign of the responses to structural shocks. We discuss recursive identification and sign identification in the following paragraphs.

A straigthforward identification scheme from the group of short-run identifying restrictions that to date has commonly been used, cf. e.g. Lütkepohl et al. (2015) and Belongia and Ireland (2015), consists of orthogonalizing impulse responses based on the Choleski decomposition of the estimated covariance matrix. While the technical implementation of this method is easy, cf. Corollary 2.8 and Section 3.2, it has to be noted that it implies a recursive ordering of the modelled variables. This means for example, that a shock in the last structural innovation contemporaneously only affects the economic variable that is ordered last in the VAR. And it also means, that for different orderings of the variables different impulse response functions are obtained. Criticism like this has lead to some of the other identifying approaches becoming more important, cf. e.g. Uhlig (2005).

Uhlig (2005) suggests identification by sign restrictions as a method of using as few identifying assumptions as possible for identifying certain structural shocks. 
The paper treats the case of identifying monetary policy shocks, for which it is suggested to use as few assumptions as possible. The argument is that the smaller the number of identifying assumptions is, the more likely it is to have agreement on them among economists. Also it should be tried not to influence the results of an econometric analysis too much by imposing too much structure on the model. The assumptions are stated in terms of the algebraic sign of the responses to a contractionary monetary policy shock: Increases in prices and nonborrowed reserves and decreases in the federal funds rate a certain number of periods after the shock are considered not to be consistent with a contractionary monetary policy shock. What is still left to decide then, of course, is the exact number of periods for which to check these conditions. Under this approach many different reparametrizations of the reduced-form model to a structural model are generated, and the resulting impulse responses are checked for meeting the identifying assumptions. Thus, contrary to e.g. recursively identified models, the researcher is left with not one, but typically a set of different structural models that meet the assumptions. Uhlig (2005) illustrates this method with empirical data and compares it to the approach of recursive identification. He concludes that contractionary monetary shocks have no clear effect on real GDP. We employ the same data set in Chapter 4.

The focus of this thesis is on the study of different model selection and averaging criteria. For this reason we keep one fixed parametrization throughout this thesis, namely the Choleski orthogonalization. This is especially important since the empirical data used in this thesis has been studied in other publications before, all of which have used that same identifying approach, and we will make comparisons between their results and ours. In Chapter 4 the criteria from Chapters 2 and 3 are used for estimating impulse response functions from empirical data. Their usefulness for practical applications is demonstrated by comparing the results with those obtained from the application of other commonly used model criteria.

Structure The following two chapters provide the theory for two different classes of model criteria, FIC - Chapter 2 - and smoothed averaging - Chapter 3 - while Chapter 4 is an application of these and other criteria to empirical data. Throughout the thesis we will compare the introduced model criteria to other well known ones like AIC, BIC, HQ model selection, the specifics of which are mostly given in the beginning sections of Chapters 3 and 4 , and also jackknife model averaging.

In Chapter 2 we detail the idea of directing estimation towards a focus parameter, extending the theory of the FIC to stationary multivariate time series models. The FIC concept leads us to define a set of model selection, as well as averaging estimators, with and without bias correction. We determine the asymptotic properties and elaborate on the role of the locally misspecified parameters. Monte Carlo simulations show that our Focused Information Criterion performs comparably to both AIC and BIC selection procedures as well as model averaging procedures based on their smoothed counterparts. In Chapter 3 we show that the smoothed model averaging versions of AIC and BIC model selection, sAIC, sBIC, have asymptotic properties similar to their selection counterparts. We explain that there is actually a whole class of sAIC and sBIC estimators governed by a scaling parameter, a fact that has not been clearly expressed in the literature so far. 


\section{CHAPTER 1}

And we derive a bootstrap procedure for determining confidence regions around the averaging estimates. The theoretical results are illustrated with Monte Carlo simulations that also show the estimators' MSEs and effective coverages of the constructed confidence bands. Chapter 4 revisits three previous studies on policy analysis and applies the criteria from the previous chapters along with some other model criteria, that have not been routinely used on macroeconomic data so far, to show their usefulness in practical applications. We elaborate on the effect of sampling frequency and check the robustness of the conclusions drawn from impulse response analyses with structural VAR models. Chapter 5 concludes.

Notation Our notation is largely based on Abadir and Magnus (2002). In particular $\stackrel{d}{\longrightarrow}$ and $\stackrel{p}{\longrightarrow}$ signify convergence in distribution and convergence in probability, respectively. $T$ denotes the sample size of a time series. 


\section{Chapter 2}

\section{A Focused Information Criterion for Locally Misspecified VARs}

This chapter investigates the properties of the Focused Information Criterion (FIC) as pioneered by Claeskens and Hjort (2003), and the plug-in average in (vector) autoregressive models with local-to-zero misspecification. The FIC method has the advantage of focusing on a quantity of interest rather than aiming at overall model fit. Any (sufficiently regular) function of the parameters can be used as a quantity of interest. We determine the asymptotic properties and elaborate on the role of the locally misspecified parameters. In particular, we show that the inability to consistently estimate locally misspecified parameters translates into sub-optimal selection and averaging. We apply this framework to impulse response analysis. Monte Carlo simulations show that our Focused Information Criterion performs comparably to both AIC and BIC selection procedures as well as model averaging procedures based on their smoothed counterparts. ${ }^{1}$

\section{$2.1 \quad$ Introduction}

The motivation for this chapter stems from Hansen (2005). The author considers a Gausssian ARMA $(1,1)$ model approximated by $\operatorname{AR}(k)$ models with $k \in$ $\left\{0,1, \cdots, k_{\max }\right\}$ and is interested in the impulse responses. Table 1 of Hansen (2005) shows that the MSE-minimizing AR order depends strongly on parameter values and the impulse response horizon. An extreme case is the specification $y_{t}=0.5 y_{t-1}+\epsilon_{t}-0.9 \epsilon_{t-1}$. The MSE-minimizing autoregressive orders equal 0 and 10 for the impulse responses at horizon 2 and 6 , respectively. Ivanov and Kilian (2005) report a similar issue in a VAR setting. They simulate VAR processes similar to those often found in empirical work, and rank different model

\footnotetext{
${ }^{1}$ This chapter is based on Lohmeyer et al. (2018).
} 


\section{CHAPTER 2}

selection criteria (AIC, BIC, HQ and serial correlation tests) based on the MSE of the estimated impulse responses implied by the selected model. A uniformly best criterion was not found. This might be expected since information criteria like $\mathrm{AIC}$ and BIC aim at global model fit and do not take into account the quantity of interest (such as the impulse response at a particular horizon). The Focused Information Criterion introduced by Claeskens and Hjort (2003) does take into account the interest of the researcher. Hansen (2005) acknowledged the opportunities for the FIC for the estimation of impulse responses when he remarked based on simulation outcomes: "The message from Tables 2 and 3 is that the FIC is an intriguing challenger to existing model selection methods and deserves attention and scrutiny". A theoretical justification of these simulation results was not provided.

We develop a theoretical framework starting from a vector autoregression where part of the coefficients are local-to-zero, i.e. declining to zero at a rate of $T^{-1 / 2}$ with $T$ denoting sample size. This setup is fundamentally different from a static one, because dynamic properties are also varying with sample size. It has the advantage that the omitted variable bias for the local-to-zero parameters does not diverge with increasing sample size, and thus it lends itself well to illustrating the typical estimator bias-variance tradeoff in model selection, as Hjort and Claeskens (2003b) argue in their rejoinder. The idea of defining such local-to-zero alternatives is related to local power analysis for hypothesis testing, cf. McManus (1991) for a brief discussion of its history. More recently Leeb and Pötscher (2005) have used the setup of a linear regression model with two regressors, with the coefficient of the second regressor declining to zero at rate $T^{-1 / 2}$. They show that the post-model-selection estimator's finite-sample distribution cannot be estimated and that any estimator of its asymptotic distribution cannot be consistent uniformly. The authors have published several other papers to analyze post-modelselection estimators within such local-to-zero settings. Dynamic models under local-to-zero misspecification were discussed in Claeskens et al. (2007) and Rohan and Ramanathan (2011). Both papers first derive the asymptotic results in a setting without local misspecification and subsequently introduce the misspecification (see p. 363 of Claeskens et al. (2007) and Equation (8) on p. 221 of Rohan and Ramanathan (2011)). The theoretical implications of this two-step procedure are not completely clear.

Building on ideas from Claeskens and Hjort (2003), Claeskens and Hjort (2008) and Liu (2015), we propose an estimator that can be used for both model selection and model averaging. This estimator is fairly general as it only requires the parameters of interest to be a sufficiently smooth transformation of the model's parameters. The results are subsequently applied to the specific case of impulse responses. A slight generalization of a theorem by Liu (2015) enables us to not only construct confidence intervals for a specific horizon, but to also construct confidence bands for multiple horizons. Additionally, we provide an in-depth discussion on the role of the local-to-zero parameters. These parameters cannot be estimated consistently, and we show that as a consequence the FIC and plug-in averages do not fully minimize the asymptotic mean squared error asymptotically.

The original ideas of Claeskens and Hjort (2003) are extended by Claeskens 
and Hjort (2008), and have been applied to various settings. Liu (2015) considers the linear regression setup and derives asymptotically valid confidence intervals. DiTraglia (2016) provides results for generalized method of moments estimation. Liu and Kuo (2016) consider predictive regressions.

The remainder of this chapter is organized as follows. Section 2.2 presents the model framework, the estimation procedure, and the asymptotic properties of (1) the parameter estimates, (2) the feasible FIC, and (3) the elements of the weighting matrix. A discussion and illustration of the consequences of the inconsistent estimation of the local-to-zero parameter follows. Our theoretical findings are subsequently supported by various Monte Carlo simulations in Section 2.3. Section 2.4 concludes. The mathematical proofs are presented in the Appendix. The choice of variable names is kept close to that of Liu (2015) to enhance comparability.

\subsection{Theory}

\subsubsection{The Model Framework}

Let the $K$-dimensional multiple time series $\left\{\left\{\boldsymbol{y}_{T, t}\right\}_{t=-\infty}^{\infty}\right\}_{T=1}^{\infty}$ constitute a vector triangular array generated by the vector autoregressive (VAR) processes

$\boldsymbol{y}_{T, t}=\boldsymbol{B}_{1} \boldsymbol{y}_{T, t-1}+\ldots+\boldsymbol{B}_{p_{1}} \boldsymbol{y}_{T, t-p_{1}}+\frac{\boldsymbol{\Delta}_{1}}{\sqrt{T}} \boldsymbol{y}_{T, t-p_{1}-1}+\ldots+\frac{\boldsymbol{\Delta}_{p_{2}}}{\sqrt{T}} \boldsymbol{y}_{T, t-p_{1}-p_{2}}+\boldsymbol{u}_{t}$,

where $\boldsymbol{B}_{i}, i=1,2, \ldots, p_{1}$ and $\boldsymbol{\Delta}_{i}, i=1,2, \ldots, p_{2}$ are $(K \times K)$ coefficient matrices. Equation (2.1) differs in one important aspect from the usual VAR specifications, namely some of the coefficient matrices are premultiplied by $T^{-1 / 2}$ with $T$ denoting sample size. This local-to-zero misspecification causes different dynamics for every $T$. Mathematically, this decay rate will prove to be crucial for the development of the asymptotic theory because it prevents the omitted variable bias from diverging with increasing sample size. Intuitively, we could think of this model specification as expressing a degree of uncertainty concerning the true lag order. The VAR process includes $p:=p_{1}+p_{2}$ lags for finite $T$, yet asymptotically only a $\operatorname{VAR}\left(p_{1}\right)$ remains. This can be interpreted as exploring a shrinking neighborhood of the $\operatorname{VAR}\left(p_{1}\right)$ model.

\subsubsection{Parameter Estimation and Asymptotics}

To simplify notation we collect all the parameters in the matrices

$$
\boldsymbol{B}=\left(\boldsymbol{B}_{1}, \boldsymbol{B}_{2}, \cdots, \boldsymbol{B}_{p_{1}}\right), \quad \boldsymbol{C}_{T}=\left(\boldsymbol{\Delta}_{1}, \boldsymbol{\Delta}_{2}, \cdots, \boldsymbol{\Delta}_{p_{2}}\right) / \sqrt{T}=\boldsymbol{\Delta} / \sqrt{T},
$$




\section{CHAPTER 2}

and define $\boldsymbol{\Theta}_{T}=\left(\boldsymbol{B}, \boldsymbol{C}_{T}\right)$ of dimensions $K \times K p$. Similarly to Lütkepohl (2005) we also stack the observations over time to obtain

$$
\begin{aligned}
& \boldsymbol{Y}_{T}:=\left(\boldsymbol{y}_{T, 1}, \boldsymbol{y}_{T, 2}, \ldots, \boldsymbol{y}_{T, T}\right) \quad(K \times T), \\
& \boldsymbol{z}_{T, t}:=\left(\begin{array}{c}
\boldsymbol{y}_{T, t} \\
\boldsymbol{y}_{T, t-1} \\
\vdots \\
\boldsymbol{y}_{T, t-p+1}
\end{array}\right) \quad(K p \times 1), \\
& \boldsymbol{Z}_{T}:=\left(\boldsymbol{z}_{T, 0}, \boldsymbol{z}_{T, 1}, \ldots, \boldsymbol{z}_{T, T-1}\right) \quad(K p \times T), \\
& \boldsymbol{U}:=\left(\boldsymbol{u}_{1}, \boldsymbol{u}_{2}, \ldots, \boldsymbol{u}_{T}\right) \quad(K \times T) .
\end{aligned}
$$

The model can now be expressed as $\boldsymbol{Y}_{T}=\boldsymbol{\Theta}_{T} \boldsymbol{Z}_{T}+\boldsymbol{U}$. A variety of approximating models can be considered but we will restrict our attention to models that use the same lag order in every equation (also see Remark 2.5 for further details). Using the same lag order in the cross-section is common practice and will decrease the notational burden. Selection matrices are used to relate all estimators to the estimator using $p$ lags. That is, for some integer $m$ such that $p_{1} \leq m \leq p$ we define

$$
\begin{array}{rlr}
\boldsymbol{L} & :=\boldsymbol{L}^{(1)} \otimes \boldsymbol{I}_{K}, \text { with } \boldsymbol{L}^{(1)}=\left(\begin{array}{c}
\boldsymbol{I}_{p_{1}} \\
\boldsymbol{O}_{p_{2} \times p_{1}}
\end{array}\right) & \left(K p \times K p_{1}\right), \\
\boldsymbol{S}_{0} & :=\boldsymbol{S}_{0}^{(1)} \otimes \boldsymbol{I}_{K}, \text { with } \boldsymbol{S}_{0}^{(1)}=\left(\begin{array}{c}
\mathbf{O}_{p_{1} \times p_{2}} \\
\boldsymbol{I}_{p_{2}}
\end{array}\right) & \left(K p \times K p_{2}\right), \\
\boldsymbol{S}_{m} & :=\boldsymbol{S}_{m}^{(1)} \otimes \boldsymbol{I}_{K}, \text { with } \boldsymbol{S}_{m}^{(1)}=\left(\begin{array}{c}
\boldsymbol{I}_{m} \\
\mathbf{O}_{(p-m) \times m}
\end{array}\right) & (K p \times K m), \\
\boldsymbol{\Pi}_{m}^{\prime}:=\boldsymbol{\Pi}_{m}^{\prime(1)} \otimes \boldsymbol{I}_{K}, \text { with } \boldsymbol{\Pi}_{m}^{\prime(1)}=\left(\begin{array}{c}
\boldsymbol{I}_{m-p_{1}} \\
\mathbf{O}_{(p-m) \times\left(m-p_{1}\right)}
\end{array}\right) & \left(K p_{2} \times K\left(m-p_{1}\right)\right) .
\end{array}
$$

The Kronecker products with $\boldsymbol{I}_{K}$ are a direct consequence of estimating all equations with the same lag order. The regressor matrix for the estimation of the $\operatorname{VAR}(m)$ model is $\boldsymbol{Z}_{T, m}=\boldsymbol{S}_{m}^{\prime} \boldsymbol{Z}_{T}$. The implied OLS estimator of the $(K \times K m)$ matrix $\boldsymbol{\Theta}_{T, m}=\boldsymbol{\Theta}_{T} \boldsymbol{S}_{m}$ can be written as, cf. Equation (2.16),

$$
\begin{aligned}
\hat{\boldsymbol{\Theta}}_{T, m}=\boldsymbol{\Theta}_{T, m}+\boldsymbol{C}_{T}\left(\boldsymbol{I}_{K p_{2}}-\boldsymbol{\Pi}_{m}^{\prime} \boldsymbol{\Pi}_{m}\right) & \boldsymbol{S}_{0}^{\prime} \boldsymbol{Z}_{T} \boldsymbol{Z}_{T, m}^{\prime}\left(\boldsymbol{Z}_{T, m} \boldsymbol{Z}_{T, m}^{\prime}\right)^{-1} \\
& +\boldsymbol{U} \boldsymbol{Z}_{T, m}^{\prime}\left(\boldsymbol{Z}_{T, m} \boldsymbol{Z}_{T, m}^{\prime}\right)^{-1}
\end{aligned}
$$

Rearranging and multiplying by $\sqrt{T}$ yields

$$
\begin{gathered}
\sqrt{T}\left(\hat{\boldsymbol{\Theta}}_{T, m}-\boldsymbol{\Theta}_{T, m}\right)=\underbrace{\sqrt{T} \boldsymbol{C}_{T}}_{\boldsymbol{\Delta}}\left(\boldsymbol{I}_{K p_{2}}-\boldsymbol{\Pi}_{m}^{\prime} \boldsymbol{\Pi}_{m}\right) \boldsymbol{S}_{0}^{\prime}\left(\frac{1}{T} \boldsymbol{Z}_{T} \boldsymbol{Z}_{T}^{\prime}\right) \boldsymbol{S}_{m} \\
\times\left[\boldsymbol{S}_{m}^{\prime}\left(\frac{1}{T} \boldsymbol{Z}_{T} \boldsymbol{Z}_{T}^{\prime}\right) \boldsymbol{S}_{m}\right]^{-1}+\left(\frac{1}{\sqrt{T}} \boldsymbol{U} \boldsymbol{Z}_{T}^{\prime}\right) \boldsymbol{S}_{m}\left[\boldsymbol{S}_{m}^{\prime}\left(\frac{1}{T} \boldsymbol{Z}_{T} \boldsymbol{Z}_{T}^{\prime}\right) \boldsymbol{S}_{m}\right]^{-1},
\end{gathered}
$$

and it can be seen that the $T^{1 / 2}$-consistency of the estimator precisely matches the decay rate of $T^{-1 / 2}$ in the elements of the parameter matrix $\boldsymbol{C}_{T}$. As a final 
step we apply the vec operator to transform the parameter matrices into a single parameter vector,

$$
\begin{aligned}
\sqrt{T} & \left(\hat{\boldsymbol{\theta}}_{T, m}-\boldsymbol{\theta}_{T, m}\right) \\
& =\left(\left[\boldsymbol{S}_{m}^{\prime}\left(\frac{1}{T} \boldsymbol{Z}_{T} \boldsymbol{Z}_{T}^{\prime}\right) \boldsymbol{S}_{m}\right]^{-1} \boldsymbol{S}_{m}^{\prime}\left(\frac{1}{T} \boldsymbol{Z}_{T} \boldsymbol{Z}_{T}^{\prime}\right) \boldsymbol{S}_{0}\left(\boldsymbol{I}_{K p_{2}}-\boldsymbol{\Pi}_{m}^{\prime} \boldsymbol{\Pi}_{m}\right) \otimes \boldsymbol{I}_{K}\right) \boldsymbol{\delta} \\
& +\left(\left[\boldsymbol{S}_{m}^{\prime}\left(\frac{1}{T} \boldsymbol{Z}_{T} \boldsymbol{Z}_{T}^{\prime}\right) \boldsymbol{S}_{m}\right]^{-1} \boldsymbol{S}_{m}^{\prime} \otimes \boldsymbol{I}_{K}\right) \frac{1}{\sqrt{T}} \sum_{t=1}^{T} \operatorname{vec}\left(\boldsymbol{u}_{t} \boldsymbol{z}_{T, t-1}^{\prime}\right)
\end{aligned}
$$

where $\hat{\boldsymbol{\theta}}_{T, m}=\operatorname{vec}\left(\hat{\boldsymbol{\Theta}}_{T, m}\right), \boldsymbol{\theta}_{T, m}=\operatorname{vec}\left(\boldsymbol{\Theta}_{T, m}\right)$, and $\boldsymbol{\delta}=\operatorname{vec}(\boldsymbol{\Delta})$. Equation $(2.6)$ depends on (1) various selection matrices, (2) the random matrix $\frac{1}{T} \boldsymbol{Z}_{T} \boldsymbol{Z}_{T}^{\prime}=$ $\frac{1}{T} \sum_{t=1}^{T} \boldsymbol{z}_{T, t-1} \boldsymbol{z}_{T, t-1}^{\prime}$, and (3) the random vector $\frac{1}{\sqrt{T}} \sum_{t=1}^{T} \operatorname{vec}\left(\boldsymbol{u}_{t} \boldsymbol{z}_{T, t-1}^{\prime}\right)$. The latter two rescaled sums are typically encountered in laws of large numbers and central limit theorems, respectively. The following three assumptions guarantee that such theorems are applicable.

Assumption 2.1. The sequence $\left\{\boldsymbol{u}_{t}\right\}$ of random $K$-vectors is an independent and identically distributed sequence with mean vector zero, a positive definite covariance matrix $\mathrm{E}\left(\boldsymbol{u}_{t} \boldsymbol{u}_{t}^{\prime}\right)=\boldsymbol{\Sigma}$, and there exists a $c>0$ such that $\mathrm{E}\left|u_{i t} u_{j t} u_{k t} u_{m t}\right|<c<\infty$ for $i, j, k, m=1,2, \ldots, K$.

Assumption 2.2. For all $|z| \geq 1$ and $\forall T \in \mathbb{N}$ :

$\operatorname{det}\left(\boldsymbol{B}_{T}(z)\right)=\operatorname{det}\left(\boldsymbol{I}_{K} z^{p}-\boldsymbol{B}_{1} z^{p-1}-\ldots-\boldsymbol{B}_{p_{1}} z^{p_{2}}-\frac{\boldsymbol{\Delta}_{1}}{\sqrt{T}} z^{p_{2}-1}-\ldots-\frac{\boldsymbol{\Delta}_{p_{2}}}{\sqrt{T}}\right) \neq 0$.

Assumption 2.3. $\operatorname{det}\left(\boldsymbol{B}_{\infty}(z)\right)=\operatorname{det}\left(\boldsymbol{I}_{K} z^{p_{1}}-\boldsymbol{B}_{1} z^{p_{1}-1}-\ldots-\boldsymbol{B}_{p_{1}}\right) \neq 0$ for all $|z| \geq 1$.

Assumption 2.1 provides moment bounds and independence between the innovation $\boldsymbol{u}_{t}$ and its past. This latter property is exploited to apply limit theorems for martingale differences. ${ }^{2}$ Assumptions 2.2 and 2.3 require the vector autoregressive process to be stationary for every finite $T$ and also in the absence of local misspecification. The asymptotic properties of the OLS estimators are stated in the following Theorem 2.4.

Theorem 2.4 (Asymptotic Normality of the Least Squares Estimator). Let Assumptions 2.1 - 2.3 hold. Then

(a) In the limit $T \rightarrow \infty$, we have for any $m \in \mathcal{M}=\left\{p_{1}, p_{1}+1, \ldots, p\right\}$,

$$
\sqrt{T}\left(\hat{\boldsymbol{\theta}}_{T, m}-\boldsymbol{\theta}_{T, m}\right) \stackrel{d}{\longrightarrow} \boldsymbol{A}_{m} \boldsymbol{\delta}+\left(\left[\boldsymbol{S}_{m}^{\prime} \boldsymbol{\Omega} \boldsymbol{S}_{m}\right]^{-1} \boldsymbol{S}_{m}^{\prime} \otimes \boldsymbol{I}_{K}\right) \boldsymbol{R}
$$

\footnotetext{
${ }^{2}$ The requirement of i.i.d. innovations can be relaxed to the assumption that $\left\{\boldsymbol{u}_{t}\right\}$ is a martingale difference sequence. Formally, let $\mathcal{F}_{t}=\sigma\left(\boldsymbol{u}_{s},-\infty<s \leq t\right)$ denote the sigma field generated by the innovations up to and including time $t$. Our results remain valid if the conditions $\mathrm{E}\left(\boldsymbol{u}_{t}\right)=\mathbf{0}$ and $\mathrm{E}\left(\boldsymbol{u}_{t} \boldsymbol{u}_{t}^{\prime}\right)=\boldsymbol{\Sigma}$ are replaced by $\mathrm{E}\left(\boldsymbol{u}_{t} \mid \mathcal{F}_{t-1}\right)=\mathbf{0}$ and $\mathrm{E}\left(\boldsymbol{u}_{t} \boldsymbol{u}_{t}^{\prime} \mid \mathcal{F}_{t-1}\right)=\boldsymbol{\Sigma}$, respectively.
} 


\section{CHAPTER 2}

$$
\sim \mathrm{N}\left(\boldsymbol{A}_{m} \boldsymbol{\delta},\left[\boldsymbol{S}_{m}^{\prime} \boldsymbol{\Omega} \boldsymbol{S}_{m}\right]^{-1} \otimes \boldsymbol{\Sigma}\right)
$$

with $\boldsymbol{\Omega}=\operatorname{plim} \frac{1}{T} \sum_{t=1}^{T} \boldsymbol{z}_{T, t-1} \boldsymbol{z}_{T, t-1}^{\prime}, \boldsymbol{R} \sim \mathrm{N}(\mathbf{0}, \boldsymbol{\Omega} \otimes \boldsymbol{\Sigma})$, and $\boldsymbol{A}_{m}=$ $\left[\boldsymbol{S}_{m}^{\prime} \boldsymbol{\Omega} \boldsymbol{S}_{m}\right]^{-1} \boldsymbol{S}_{m}^{\prime} \boldsymbol{\Omega} \boldsymbol{S}_{0}\left(\boldsymbol{I}_{K p_{2}}-\boldsymbol{\Pi}_{m}^{\prime} \boldsymbol{\Pi}_{m}\right) \otimes \boldsymbol{I}_{K}$

(b) Let $\hat{\boldsymbol{u}}_{T, t}^{m}$ denote the OLS residuals from the estimation of a VAR(m). Consider $\hat{\boldsymbol{\Sigma}}^{m}=\frac{1}{T} \sum_{t=1}^{T} \hat{\boldsymbol{u}}_{T, t}^{m} \hat{\boldsymbol{u}}_{T, t}^{m \prime}$ as an estimator for $\boldsymbol{\Sigma}$. The result in part (a) can be strengthened to joint asymptotic normality with the covariance matrix estimator $\hat{\boldsymbol{\Sigma}}^{m}$,

$$
\left[\begin{array}{c}
\sqrt{T}\left(\hat{\boldsymbol{\theta}}_{T, m}-\boldsymbol{\theta}_{T, m}\right) \\
\sqrt{T} \operatorname{vech}\left(\hat{\boldsymbol{\Sigma}}^{m}-\boldsymbol{\Sigma}\right)
\end{array}\right] \stackrel{d}{\longrightarrow} \mathrm{N}\left(\left[\begin{array}{c}
\boldsymbol{A}_{m} \\
\mathbf{O}
\end{array}\right] \boldsymbol{\delta},\left[\begin{array}{cc}
{\left[\boldsymbol{S}_{m}^{\prime} \boldsymbol{\Omega S}_{m}\right]^{-1} \otimes \boldsymbol{\Sigma}} & \mathbf{O} \\
\mathbf{O} & \boldsymbol{\Xi}_{22}
\end{array}\right]\right) .
$$

The matrix $\boldsymbol{\Xi}_{22}$ is specified in the Appendix.

(c) The estimator convergence as discussed in parts (a) and (b) of this theorem is also a joint convergence across different $m \in \mathcal{M}$. That is, for $\left\{i_{1}, i_{2}, \ldots, i_{M}\right\} \in \mathcal{M}$, any $m \in \mathcal{M}$, and $i_{1}<i_{2}<\ldots<i_{M}$, we have

$$
\left[\begin{array}{c}
\sqrt{T}\left(\hat{\boldsymbol{\theta}}_{T, i_{1}}-\boldsymbol{\theta}_{i_{1}}\right) \\
\sqrt{T}\left(\hat{\boldsymbol{\theta}}_{T, i_{2}}-\boldsymbol{\theta}_{i_{2}}\right) \\
\vdots \\
\sqrt{T}\left(\hat{\boldsymbol{\theta}}_{T, i_{M}}-\boldsymbol{\theta}_{i_{M}}\right) \\
\sqrt{T} \operatorname{vech}\left(\hat{\boldsymbol{\Sigma}}^{m}-\boldsymbol{\Sigma}\right)
\end{array}\right] \stackrel{d}{\longrightarrow}\left(\left[\begin{array}{c}
\boldsymbol{A}_{i_{1}} \\
\boldsymbol{A}_{i_{2}} \\
\vdots \\
\boldsymbol{A}_{i_{M}} \\
\mathbf{O}
\end{array}\right] \boldsymbol{\delta},\left[\begin{array}{ccccc}
\boldsymbol{V}_{i_{1} i_{1}} & \boldsymbol{V}_{i_{1} i_{2}} & \ldots & \boldsymbol{V}_{i_{1} i_{M}} & \mathbf{O} \\
\boldsymbol{V}_{i_{2} i_{1}} & \boldsymbol{V}_{i_{2} i_{2}} & \ldots & \boldsymbol{V}_{i_{2} i_{M}} & \mathbf{O} \\
\vdots & \vdots & \ddots & \vdots & \vdots \\
\boldsymbol{V}_{i_{M} i_{1}} & \boldsymbol{V}_{i_{M} i_{2}} & \ldots & \boldsymbol{V}_{i_{M} i_{M}} & \mathbf{O} \\
\mathbf{O} & \mathbf{O} & \ldots & \mathbf{O} & \boldsymbol{\Xi}_{22}
\end{array}\right]\right) .
$$

The matrices $\boldsymbol{V}_{j k}$ are given by $\boldsymbol{V}_{j k}=\left[\boldsymbol{S}_{j}^{\prime} \boldsymbol{\Omega} \boldsymbol{S}_{j}\right]^{-1} \boldsymbol{S}_{j}^{\prime} \boldsymbol{\Omega} \boldsymbol{S}_{k}\left[\boldsymbol{S}_{k}^{\prime} \boldsymbol{\Omega} \boldsymbol{S}_{k}\right]^{-1} \otimes \boldsymbol{\Sigma}$. It suffices to consider a single estimator for $\boldsymbol{\Sigma}$ because all the estimators are asymptotically equivalent.

The matrix $\boldsymbol{\Omega}$ deserves further attention. It is defined as the probability limit of the Gram matrix $\frac{1}{T} \boldsymbol{Z}_{T} \boldsymbol{Z}_{T}^{\prime}$. The proof of Theorem 2.4 reveals that this probability limit exists, and that it equals $\mathrm{E}\left(\boldsymbol{z}_{\infty, t} \boldsymbol{z}_{\infty, t}^{\prime}\right)$ where $\boldsymbol{z}_{\infty, t}$ is defined as in Equations (2.2) but being generated by the VAR without local misspecification. As an example consider the AR process defined by $y_{T, t}=\beta y_{T, t-1}+\frac{\delta_{1}}{\sqrt{T}} y_{T, t-2}+\frac{\delta_{2}}{\sqrt{T}} y_{T, t-2}+u_{t}$, that is an AR model with $p_{1}=1, p_{2}=2$ and $p=3$. In that case we obtain $\boldsymbol{\Omega}=\frac{\sigma^{2}}{1-\beta^{2}}\left[\begin{array}{ccc}1 & \beta & \beta^{2} \\ \beta & 1 & \beta \\ \beta^{2} & \beta & 1\end{array}\right]$.

Remark 2.5. The consequences of the local misspecification framework are visible in Theorem 2.4. Standard asymptotics will fail if relevant parameters are left out since the omitted variable bias will dominate asymptotically. ${ }^{3}$ The local-to-zero

\footnotetext{
${ }^{3}$ Let us consider the data generating process $y_{t}=\beta_{1} y_{t-1}+\beta_{2} y_{t-2}+u_{t}$. Suppose that we estimate an AR(1) model. The OLS parameter estimator of the first lag coefficient, say $\hat{\beta}$, satisfies $\sqrt{T}\left(\hat{\beta}-\beta_{1}\right)=\sqrt{T} \beta_{2} \frac{\frac{1}{T} \sum_{t=1}^{T} y_{t-1} y_{t-2}}{\frac{1}{T} \sum_{t=1}^{T} y_{t-1}^{2}}+\frac{\frac{1}{\sqrt{T}} \sum_{t=1}^{T} y_{t-1} u_{t}}{\frac{1}{T} \sum_{t=1}^{T} y_{t-1}^{2}}$. The first term on the RHS diverges at large $T$ for $\beta_{2} \neq 0$. The divergence rate is $\sqrt{T}$.
} 
rate of $T^{-1 / 2}$ balances this diverging behavior such that a finite asymptotic bias remains. This reasoning applies to all models that contain all the fixed parameters (i.e. the lag order should be no less than $p_{1}$ ) and leave out arbitrary parameters that are local-to-zero.

Remark 2.6. Assumption 2.2 is rather strict because it requires stationarity for all $T$ in the natural numbers. Is it even possible for any parameter combination to satisfy this assumption? We can answer this question in the affirmative for the univariate case but the result does not generalize easily to the multivariate case. For the univariate case the assumption is equivalent to bounding the modulus roots of the polynomial

$$
z^{p}-\beta_{1} z^{p-1}-\ldots-\beta_{p_{1}} z^{p_{2}}-\frac{\delta_{1}}{\sqrt{T}} z^{p_{2}-1}-\ldots-\frac{\delta_{p_{2}}}{\sqrt{T}}
$$

at below 1. Fujiwara (1916) has shown that the largest modulus root of a polynomial $a(z)=a_{0} z^{p}+a_{1} z^{p-1}+\ldots+a_{p-1} z+a_{p}$ is bounded above by $2 \max \left\{\left|a_{1} / a_{0}\right|,\left|a_{2} / a_{0}\right|^{1 / 2}, \ldots,\left|a_{p} / a_{0}\right|^{1 / p}\right\}$. The largest modulus root of the lag polynomial $\beta_{T}$ is thus bounded by

$$
2 \max \left\{\left|\beta_{1}\right|,\left|\beta_{2}\right|^{\frac{1}{2}}, \ldots,\left|\beta_{p_{1}}\right|^{\frac{1}{p_{1}}},\left|\frac{\delta_{1}}{\sqrt{T}}\right|^{\frac{1}{p_{1}+1}}, \ldots,\left|\frac{\delta_{p_{2}}}{\sqrt{T}}\right|^{\frac{1}{p}}\right\} .
$$

We deduce from Equation (2.7) that

$$
2 \max \left\{\left|\beta_{1}\right|,\left|\beta_{2}\right|^{\frac{1}{2}}, \ldots,\left|\beta_{p_{1}}\right|^{\frac{1}{p_{1}}},\left|\delta_{1}\right|^{\frac{1}{p_{1}+1}}, \ldots,\left|\delta_{p_{2}}\right|^{\frac{1}{p}}\right\}<1
$$

guarantees stationarity for all $T .^{4}$

\subsubsection{The Quantities of Interest}

The Focused Information Criterion (FIC) introduced by Claeskens and Hjort (2003) focusses on a quantity of interest rather than general model fit. Quantities of interest could be a single parameter, several parameters, or parameter transformations. Natural quantities of interest in the current dynamical setting are the impulse responses. In general, let $\boldsymbol{\mu}: \mathbb{R}^{K^{2} p+K(K+1) / 2} \rightarrow \mathbb{R}^{l}$ define the mapping from the model parameters to an $l$-dimensional focus quantity. The first $K^{2} p$ arguments of the function $\boldsymbol{\mu}$ are reserved for the conditional mean parameters, whereas the last $K(K+1) / 2$ arguments refer to the parameters in $\boldsymbol{\Sigma}$. As such we define $\boldsymbol{\sigma}=\operatorname{vech}(\boldsymbol{\Sigma})$ and $\widehat{\boldsymbol{\sigma}}=\operatorname{vech}(\widehat{\boldsymbol{\Sigma}})$, and write $\mu(\boldsymbol{\theta}, \boldsymbol{\sigma}){ }^{5}$ We additionally assume that evaluating the quantity of interest at $\boldsymbol{\mu}\left(\left(\boldsymbol{\theta}_{T, m}, \mathbf{0}_{K^{2}(p-m)}\right), \boldsymbol{\sigma}\right)$ provides the quantity of interest in the model with $m$ lags. Lemma 2.21 in the Appendix shows that this is true for impulse responses. The next theorem follows from Theorem 2.4 and the multivariate first order delta method.

\footnotetext{
${ }^{4}$ This condition is a sufficient but by no means a necessary condition. For $p_{1}=1$ and $p_{2}=1$, the model $y_{T, t}=0.7 y_{T, t-1}+\frac{0.75}{\sqrt{T}} y_{T, t-2}+u_{t}$ is stationary for all $T$ but the parameters violate the requirement based on Fujiwara's bound.

${ }^{5}$ Theorem 2.4 showed that all the $\widehat{\boldsymbol{\Sigma}}^{m}$ are asymptotically equivalent. So we will omit the superscript $m$ from now on.
} 
Theorem 2.7 (Asymptotic Normality of the Quantities of Interest). Let $\boldsymbol{\mu}: \mathbb{R}^{K^{2} p+K(K+1) / 2} \rightarrow \mathbb{R}^{l}$ have a continuous first derivative at all points $\left(\boldsymbol{\theta}_{T, m}, \mathbf{0}_{K^{2}(p-m)}, \boldsymbol{\sigma}\right)$, with $m \in \mathcal{M}$. Let $\boldsymbol{\theta}_{\infty}$ denote the parameters obtained by taking $\boldsymbol{\theta}_{T, p}$ but setting $\boldsymbol{\Delta}_{1}=\boldsymbol{\Delta}_{2}=\ldots=\boldsymbol{\Delta}_{p_{2}}=\mathbf{O}$, and define the Jacobian matrices $\boldsymbol{D}_{\theta}=\partial \boldsymbol{\mu}\left(\boldsymbol{\theta}_{\infty}, \boldsymbol{\sigma}\right) / \partial \boldsymbol{\theta}^{\prime}$ and $\boldsymbol{D}_{\sigma}=\partial \boldsymbol{\mu}\left(\boldsymbol{\theta}_{\infty}, \boldsymbol{\sigma}\right) / \partial \boldsymbol{\sigma}^{\prime}$. For $\boldsymbol{D}_{\theta}$ and $\boldsymbol{D}_{\sigma}$ not having zero rows, under Assumptions 2.1 - 2.3, and as $T \rightarrow \infty$,

$$
\begin{aligned}
& \sqrt{T}\left(\boldsymbol{\mu}\left(\left(\hat{\boldsymbol{\theta}}_{T, m}, \mathbf{0}_{K^{2}(p-m)}\right), \hat{\boldsymbol{\sigma}}\right)-\boldsymbol{\mu}\left(\boldsymbol{\theta}_{T, p}, \boldsymbol{\sigma}\right)\right) \\
& \stackrel{d}{\longrightarrow} \mathrm{N}\left(\boldsymbol{D}_{\theta} \boldsymbol{C}_{m} \boldsymbol{\delta}, \boldsymbol{D}_{\theta} \boldsymbol{P}_{m}(\boldsymbol{\Omega} \otimes \boldsymbol{\Sigma}) \boldsymbol{P}_{m} \boldsymbol{D}_{\theta}^{\prime}+\boldsymbol{D}_{\sigma} \boldsymbol{\Xi}_{22} \boldsymbol{D}_{\sigma}^{\prime}\right),
\end{aligned}
$$

with $\boldsymbol{P}_{m}=\boldsymbol{S}_{m}\left[\boldsymbol{S}_{m}^{\prime} \boldsymbol{\Omega} \boldsymbol{S}_{m}\right]^{-1} \boldsymbol{S}_{m}^{\prime} \otimes \boldsymbol{I}_{K}$, which is of size $K^{2} p \times K^{2} p$, and $\boldsymbol{C}_{m}=\left(\boldsymbol{S}_{m}\left[\boldsymbol{S}_{m}^{\prime} \boldsymbol{\Omega} \boldsymbol{S}_{m}\right]^{-1} \boldsymbol{S}_{m}^{\prime} \boldsymbol{\Omega}-\boldsymbol{I}_{K p}\right) \boldsymbol{S}_{0}\left(\boldsymbol{I}_{K p_{2}}-\boldsymbol{\Pi}_{m}^{\prime} \boldsymbol{\Pi}_{m}\right) \otimes \boldsymbol{I}_{K}: \quad K^{2} p \times K^{2} p_{2}$.

We define the impulse response at horizon $h$ as the $h$ 'th coefficient matrix of the $\operatorname{MA}(\infty)$ representation $\boldsymbol{y}_{t}=\sum_{h=0}^{\infty} \boldsymbol{\Phi}_{h} \boldsymbol{u}_{t-h}$ with $\boldsymbol{\Phi}_{0}=\boldsymbol{I}_{K}$, hence considering its vectorized form $\boldsymbol{\mu}: \mathbb{R}^{K^{2} p+K(K+1) / 2} \rightarrow \mathbb{R}^{K^{2}}$. Theorem 2.7 can be applied if the Jacobian matrices $\boldsymbol{D}_{\theta}$ and $\boldsymbol{D}_{\sigma}$ are known. Lütkepohl (1990) lists these Jacobian matrices for the impulse responses, the orthogonalized impulse responses, the accumulated responses, the total accumulated responses, and the forecast error variance decomposition. For applying his Proposition 1 in the following, we define the $\left(K(K+1) / 2 \times K^{2}\right)$ elimination matrix $\boldsymbol{L}_{K}$ and the commutation matrix $\boldsymbol{K}_{K K}$ such that for any $(K \times K)$ matrix $\boldsymbol{G}$ : $\operatorname{vech}(\boldsymbol{G})=\boldsymbol{L}_{K} \operatorname{vec}(\boldsymbol{G})$, and $\boldsymbol{K}_{K K} \operatorname{vec}(\boldsymbol{G})=$ $\operatorname{vec}\left(\boldsymbol{G}^{\prime}\right)$.

The specific case of the (orthogonalized) impulse responses is highlighted in the following Corollary, which follows with Proposition 1 of Lütkepohl (1990).

Corollary 2.8 (An Application to Impulse Responses). Let $\boldsymbol{A}_{\infty}$ denote the $(K p \times K p)$ companion matrix related to the process in which the misspecification coefficients have been set to zero, $\boldsymbol{J}=\left(\boldsymbol{I}_{K}, \mathbf{O}, \cdots, \mathbf{O}\right)$ a matrix of dimensions $K \times$ $K p$ and the Choleski factors be given by $\boldsymbol{\Sigma}=\boldsymbol{P} \boldsymbol{P}^{\prime}$. Then, under the assumptions of Theorem 2.7,

(a) The asymptotic distribution of the estimated impulse response at horizon $h$, $\hat{\boldsymbol{\Phi}}_{h}$, follows

$$
\sqrt{T} \operatorname{vec}\left(\hat{\boldsymbol{\Phi}}_{h}-\boldsymbol{\Phi}_{h}\right) \stackrel{d}{\longrightarrow} \mathrm{N}\left(\boldsymbol{G}_{h} \boldsymbol{C}_{m} \boldsymbol{\delta}, \boldsymbol{G}_{h} \boldsymbol{P}_{m}(\boldsymbol{\Omega} \otimes \boldsymbol{\Sigma}) \boldsymbol{P}_{m} \boldsymbol{G}_{h}^{\prime}\right),
$$

where $\boldsymbol{G}_{h}=\partial \operatorname{vec}\left(\boldsymbol{\Phi}_{h}\right) / \partial \boldsymbol{\theta}=\sum_{j=0}^{h-1} \boldsymbol{J}\left(\boldsymbol{A}_{\infty}\right)^{h-1-j} \otimes \boldsymbol{\Phi}_{j}: \quad K^{2} \times K^{2} p$.

(b) The asymptotic distribution of the estimated orthogonalized impulse response at horizon $h, \hat{\boldsymbol{\Psi}}_{h}$, follows

$$
\sqrt{T} \operatorname{vec}\left(\hat{\boldsymbol{\Psi}}_{h}-\boldsymbol{\Psi}_{h}\right) \stackrel{d}{\longrightarrow} \mathrm{N}\left(\boldsymbol{F}_{h} \boldsymbol{C}_{m} \boldsymbol{\delta}, \boldsymbol{F}_{h} \boldsymbol{P}_{m}(\boldsymbol{\Omega} \otimes \boldsymbol{\Sigma}) \boldsymbol{P}_{m} \boldsymbol{F}_{h}^{\prime}+\overline{\boldsymbol{F}}_{h} \boldsymbol{\Xi}_{22} \overline{\boldsymbol{F}}_{h}^{\prime}\right),
$$

where $\boldsymbol{F}_{0}=\mathbf{O}$ and $\boldsymbol{F}_{h}=\left(\boldsymbol{P}^{\prime} \otimes \boldsymbol{I}_{K}\right) \boldsymbol{G}_{h}$ for $h>0$. We have $\overline{\boldsymbol{F}}_{h}=\left(\boldsymbol{I}_{K} \otimes \boldsymbol{\Phi}_{h}\right) \boldsymbol{H}$ with $\boldsymbol{H}=\partial \operatorname{vec}(\boldsymbol{P}) / \partial \boldsymbol{\sigma}^{\prime}=\boldsymbol{L}_{K}^{\prime}\left[\boldsymbol{L}_{K}\left(\boldsymbol{I}_{K^{2}}+\boldsymbol{K}_{K K}\right)\left(\boldsymbol{P} \otimes \boldsymbol{I}_{K}\right) \boldsymbol{L}_{K}^{\prime}\right]^{-1}$ for all $h$. 
Remark 2.9. The first order delta method is invalid if either $\boldsymbol{D}_{\theta}$ or $\boldsymbol{D}_{\sigma}$ has zero rows. It is well-documented in the literature that $\boldsymbol{D}_{\theta}$ can have zero rows for specific parameter combinations when impulse responses are considered. We refer to Lütkepohl (1990) and Benkwitz et al. (2000) for details.

\section{Model Selection: The Focused Information Criterion (FIC)}

The intuition behind the FIC of Claeskens and Hjort (2003) is most easily understood for a univariate quantity of interest, so we temporarily assume $l=1$, and will write $\mu$ instead of $\boldsymbol{\mu}$, for example, to signify scalars. The generalization to multiple quantities is covered in Remark 2.10. Theorem 2.7 implies that the asymptotic mean squared error of the focus quantity $\mu\left(\left(\hat{\boldsymbol{\theta}}_{T, m}, \mathbf{0}_{K^{2}(p-m)}\right), \hat{\boldsymbol{\sigma}}\right)$, $\operatorname{AMSE}\left(\mu\left(\left(\hat{\boldsymbol{\theta}}_{T, m}, \mathbf{0}_{K^{2}(p-m)}\right), \hat{\boldsymbol{\sigma}}\right)\right)$ is equal to

$$
\boldsymbol{D}_{\theta}\left[\boldsymbol{C}_{m} \boldsymbol{\delta} \boldsymbol{\delta}^{\prime} \boldsymbol{C}_{m}^{\prime}+\boldsymbol{P}_{m}(\boldsymbol{\Omega} \otimes \boldsymbol{\Sigma}) \boldsymbol{P}_{m}\right] \boldsymbol{D}_{\theta}^{\prime}+\boldsymbol{D}_{\sigma} \boldsymbol{\Xi}_{22} \boldsymbol{D}_{\sigma}^{\prime}
$$

There are three contributions to the AMSE, (1) the term $\boldsymbol{D}_{\theta} \boldsymbol{C}_{m} \boldsymbol{\delta} \boldsymbol{\delta}^{\prime} \boldsymbol{C}_{m}^{\prime} \boldsymbol{D}_{\theta}^{\prime}$ is an asymptotic squared bias originating from the exclusion of local-to-zero parameters, (2) the asymptotic variance contribution $\boldsymbol{D}_{\theta} \boldsymbol{P}_{m}(\boldsymbol{\Omega} \otimes \boldsymbol{\Sigma}) \boldsymbol{P}_{m} \boldsymbol{D}_{\theta}^{\prime}$, and (3) the contribution $\boldsymbol{D}_{\sigma} \boldsymbol{\Xi}_{22} \boldsymbol{D}_{\sigma}^{\prime}$ which does not depend on the lag order $m$. Overall we face a bias-variance tradeoff when having to decide on $m$.

The FIC is an estimate of the AMSE. The quantities $\hat{\boldsymbol{\Omega}}=\frac{1}{T} \sum_{t=1}^{T} \boldsymbol{z}_{T, t} \boldsymbol{z}_{T, t}^{\prime}$ and $\hat{\boldsymbol{\theta}}_{T, p}$ provide consistent estimates for $\boldsymbol{\Omega}$ and $\boldsymbol{\theta}_{T, p}$, repectively. In view of the continuous mapping theorem,

$$
\begin{aligned}
& \hat{\boldsymbol{P}}_{m}=\boldsymbol{S}_{m}\left[\boldsymbol{S}_{m}^{\prime} \hat{\boldsymbol{\Omega}} \boldsymbol{S}_{m}\right]^{-1} \boldsymbol{S}_{m}^{\prime} \otimes \boldsymbol{I}_{K}, \text { and } \\
& \hat{\boldsymbol{C}}_{m}=\left(\boldsymbol{S}_{m}\left[\boldsymbol{S}_{m}^{\prime} \hat{\boldsymbol{\Omega}} \boldsymbol{S}_{m}\right]^{-1} \boldsymbol{S}_{m}^{\prime} \hat{\boldsymbol{\Omega}}-\boldsymbol{I}_{K p}\right) \boldsymbol{S}_{0}\left(\boldsymbol{I}_{K p_{2}}-\boldsymbol{\Pi}_{m}^{\prime} \boldsymbol{\Pi}_{m}\right) \otimes \boldsymbol{I}_{K}
\end{aligned}
$$

are consistent estimators as well. A consistent estimator for $\boldsymbol{\delta}$ is not available due to the adopted misspecification framework. We follow the existing literature (see Claeskens and Hjort (2003), Liu (2015), and Charkhi et al. (2015) among others) and use $\hat{\boldsymbol{\delta}}=\sqrt{T} \operatorname{vec}\left(\hat{\boldsymbol{\Theta}}_{T, p} \boldsymbol{S}_{0}\right)$ which satisfies ${ }^{6}$

$$
\hat{\boldsymbol{\delta}} \stackrel{d}{\longrightarrow} \boldsymbol{R}_{\delta}=\boldsymbol{\delta}+\left(\boldsymbol{S}_{0}^{\prime} \boldsymbol{\Omega}^{-1} \otimes \boldsymbol{I}_{K}\right) \boldsymbol{R} \sim \mathrm{N}\left(\boldsymbol{\delta}, \boldsymbol{S}_{0}^{\prime} \boldsymbol{\Omega}^{-1} \boldsymbol{S}_{0} \otimes \boldsymbol{\Sigma}\right) .
$$

Asymptotically, we have $\mathrm{E}\left(\hat{\boldsymbol{\delta}} \hat{\boldsymbol{\delta}}^{\prime}\right)=\boldsymbol{\delta} \boldsymbol{\delta}^{\prime}+\boldsymbol{S}_{0}^{\prime} \boldsymbol{\Omega}^{-1} \boldsymbol{S}_{0} \otimes \boldsymbol{\Sigma}$. Using the asymptotically unbiased estimate $\hat{\boldsymbol{\delta}} \hat{\boldsymbol{\delta}}^{\prime}-\boldsymbol{S}_{0}^{\prime} \hat{\boldsymbol{\Omega}}^{-1} \boldsymbol{S}_{0} \otimes \hat{\boldsymbol{\Sigma}}$ for $\boldsymbol{\delta} \boldsymbol{\delta}^{\prime}$, the FIC for the approximating model with $m$ lags is defined as

$$
\widehat{F I C}_{m}=\boldsymbol{D}_{\theta}\left[\hat{\boldsymbol{C}}_{m}\left(\hat{\boldsymbol{\delta}} \hat{\boldsymbol{\delta}}^{\prime}-\boldsymbol{S}_{0}^{\prime} \hat{\boldsymbol{\Omega}}^{-1} \boldsymbol{S}_{0} \otimes \hat{\boldsymbol{\Sigma}}\right) \hat{\boldsymbol{C}}_{m}^{\prime}+\hat{\boldsymbol{P}}_{m}(\hat{\boldsymbol{\Omega}} \otimes \hat{\boldsymbol{\Sigma}}) \hat{\boldsymbol{P}}_{m}\right] \boldsymbol{D}_{\theta}^{\prime}+\boldsymbol{D}_{\sigma} \hat{\boldsymbol{\Xi}}_{22} \boldsymbol{D}_{\sigma}^{\prime} .
$$

This estimate of the AMSE can be computed for every model, and the model with the lowest $\widehat{F I C}_{m}$ is selected. We elaborate on implications of inconsistent estimation of $\boldsymbol{\delta}$ in Section 2.2.4.

${ }^{6} \hat{\boldsymbol{\delta}}=\sqrt{T} \operatorname{vec}\left(\hat{\boldsymbol{\Theta}}_{T, p} \boldsymbol{S}_{0}\right)$ is the sample equivalent of $\boldsymbol{\delta}=\operatorname{vec}(\boldsymbol{\Delta})=\sqrt{T} \operatorname{vec}\left(\boldsymbol{C}_{T}\right)=$ $\sqrt{T} \operatorname{vec}\left(\boldsymbol{\Theta}_{T, p} \boldsymbol{S}_{0}\right)$. 


\section{CHAPTER 2}

Remark 2.10. The same procedure can be followed when $l>1$, but the AMSE becomes an $(l \times l)$ matrix. The trace or determinant are meaningful ways to describe this AMSE matrix by a scalar (see Charkhi et al. (2015)). ${ }^{7}$ The trace is computationally convenient because the overall FIC will be the sum of the individual univariate FIC contributions.

\section{Model Averaging: Plug-in Averaging}

Liu (2015) proposed a model averaging approach along the lines of the FIC. It was named plug-in averaging. We again begin by considering the case $l=1$. See Remark 2.12 for the generalization to higher dimensions. Part (b) from Theorem 2.4 implies that linear combinations of the VAR parameter estimates are also asymptotically normally distributed. Interpret the coefficients in the linear combination as weights, i.e. define $\boldsymbol{w}=\left(w_{p_{1}}, w_{p_{1}+1}, \ldots, w_{p}\right)^{\prime}$ with $\boldsymbol{w} \in \mathcal{H}=$ $\left\{\boldsymbol{w} \in[0,1]^{p_{2}+1}: \sum_{m=p_{1}}^{p} w_{m}=1\right\} \cdot{ }^{8}$ Theorem 2.11 details the asymptotic distribution of the weighted estimator

$$
\bar{\mu}(\boldsymbol{w})=\sum_{m=p_{1}}^{p} w_{m} \mu\left(\left(\hat{\boldsymbol{\theta}}_{T, m}, \mathbf{0}_{K^{2}(p-m)}\right), \hat{\boldsymbol{\sigma}}\right) .
$$

Theorem 2.11 (Asymptotic Normality of the Plug-In Estimator). Under the Assumptions of Theorem 2.7 we have for $T \rightarrow \infty$,

$$
\begin{aligned}
\sqrt{T}\left(\bar{\mu}(\boldsymbol{w})-\mu\left(\boldsymbol{\theta}_{T, p}, \boldsymbol{\sigma}\right)\right) & \stackrel{d}{\longrightarrow} \boldsymbol{D}_{\theta} \sum_{m=p_{1}}^{p} w_{m} \boldsymbol{C}_{m} \boldsymbol{\delta}+\boldsymbol{D}_{\theta} \sum_{m=p_{1}}^{p} w_{m} \boldsymbol{P}_{m} \boldsymbol{R}+\boldsymbol{D}_{\sigma} \boldsymbol{S} \\
& \sim \mathrm{N}\left(\boldsymbol{D}_{\theta} \sum_{m=p_{1}}^{p} w_{m} \boldsymbol{C}_{m} \boldsymbol{\delta}, \boldsymbol{V}+\boldsymbol{D}_{\sigma} \boldsymbol{\Xi}_{22} \boldsymbol{D}_{\sigma}^{\prime}\right)
\end{aligned}
$$

with $\boldsymbol{V}=\sum_{m=p_{1}}^{p} \sum_{l=p_{1}}^{p} w_{m} w_{l} \boldsymbol{D}_{\theta} \boldsymbol{P}_{m}(\boldsymbol{\Omega} \otimes \boldsymbol{\Sigma}) \boldsymbol{P}_{l} \boldsymbol{D}_{\theta}^{\prime}$, and $\boldsymbol{S} \sim \mathrm{N}\left(\mathbf{0}, \boldsymbol{\Xi}_{22}\right)$.

As for the FIC, we compute the AMSE and find ${ }^{9}$

$$
\operatorname{AMSE}(\bar{\mu}(\boldsymbol{w}))=\sum_{m=p_{1}}^{p} \sum_{l=p_{1}}^{p} w_{m} \boldsymbol{D}_{\theta}\left(\boldsymbol{C}_{m} \boldsymbol{\delta} \boldsymbol{\delta}^{\prime} \boldsymbol{C}_{l}^{\prime}+\boldsymbol{P}_{m}(\boldsymbol{\Omega} \otimes \boldsymbol{\Sigma}) \boldsymbol{P}_{l}\right) \boldsymbol{D}_{\theta}^{\prime} w_{l}=\boldsymbol{w}^{\prime} \boldsymbol{\Psi} \boldsymbol{w}
$$

with the $\left(\left(p_{2}+1\right) \times\left(p_{2}+1\right)\right)$ matrix $\boldsymbol{\Psi}$ having the $(m, l)$ 'th element $\boldsymbol{\Psi}_{m, l}=$ $\boldsymbol{D}_{\theta}\left(\boldsymbol{C}_{m} \boldsymbol{\delta} \boldsymbol{\delta}^{\prime} \boldsymbol{C}_{l}^{\prime}+\boldsymbol{P}_{m}(\boldsymbol{\Omega} \otimes \boldsymbol{\Sigma}) \boldsymbol{P}_{l}\right) \boldsymbol{D}_{\theta}^{\prime}$. The optimal weight vector that minimizes the AMSE is given by $\boldsymbol{w}^{0}=\arg \min _{\boldsymbol{w} \in \mathcal{H}} \boldsymbol{w}^{\prime} \boldsymbol{\Psi} \boldsymbol{w}$. But $\boldsymbol{w}^{0}$ depends on population quantities, so by using the same estimates for population quantities as before we compute feasible weights as

$$
\hat{\boldsymbol{w}}=\arg \min _{\boldsymbol{w} \in \mathcal{H}} \boldsymbol{w}^{\prime} \hat{\boldsymbol{\Psi}}^{j} \boldsymbol{w}, \quad j \in\{\text { Biased, Bias cor }\},
$$

\footnotetext{
${ }^{7}$ Any mapping from the AMSE matrix to a scalar can be used, e.g. matrix norms could be used as well.

${ }^{8}$ We assume that we average over all the models in $\mathcal{M}=\left\{p_{1}, p_{1}+1, \ldots, p\right\}$.

${ }^{9}$ The contribution $\boldsymbol{D}_{\sigma} \boldsymbol{\Xi}_{22} \boldsymbol{D}_{\sigma}^{\prime}$ does not depend on $m$, and is therefore inconsequential for the analysis. This term will be omitted from the AMSE to allow for an easier presentation.
} 
with

$$
\begin{aligned}
\hat{\boldsymbol{\Psi}}_{m, l}^{\text {Biased }} & =\boldsymbol{D}_{\theta}\left(\hat{\boldsymbol{C}}_{m} \hat{\boldsymbol{\delta}} \hat{\boldsymbol{\delta}}^{\prime} \hat{\boldsymbol{C}}_{l}^{\prime}+\hat{\boldsymbol{P}}_{m}(\hat{\boldsymbol{\Omega}} \otimes \hat{\boldsymbol{\Sigma}}) \hat{\boldsymbol{P}}_{l}\right) \boldsymbol{D}_{\theta}^{\prime}, \\
\hat{\boldsymbol{\Psi}}_{m, l}^{\text {Bias cor }} & =\boldsymbol{D}_{\theta}\left(\hat{\boldsymbol{C}}_{m}\left(\hat{\boldsymbol{\delta}} \hat{\boldsymbol{\delta}}^{\prime}-\boldsymbol{S}_{0}^{\prime} \hat{\boldsymbol{\Omega}}^{-1} \boldsymbol{S}_{0} \otimes \hat{\boldsymbol{\Sigma}}\right) \hat{\boldsymbol{C}}_{l}^{\prime}+\hat{\boldsymbol{P}}_{m}(\hat{\boldsymbol{\Omega}} \otimes \hat{\boldsymbol{\Sigma}}) \hat{\boldsymbol{P}}_{l}\right) \boldsymbol{D}_{\theta}^{\prime} .
\end{aligned}
$$

The sole difference between the matrix elements in Equation block (2.11) is an asymptotic bias correction for $\hat{\boldsymbol{\delta}} \hat{\boldsymbol{\delta}}^{\prime}$. For both versions Equation (2.10) describes a quadratic programming problem with linear constraints. Solvers are readily available (for example 'quadprog' in Matlab and 'qprog' in Gauss). The estimator for $\boldsymbol{\delta}$ remains inconsistent and we again refer to Section 2.2.4 for a discussion of the implications.

Remark 2.12. As in Remark 2.10, we will obtain an $(l \times l)$ AMSE matrix for multiple quantities of interest. This matrix has to be summarized by a scalar. The trace again has computational benefits because the objective function will take the form $\hat{\boldsymbol{w}}=\arg \min _{\boldsymbol{w} \in \mathcal{H}} \boldsymbol{w}^{\prime}\left(\sum_{i} \hat{\boldsymbol{\Psi}}_{i}^{j}\right) \boldsymbol{w}$ with the matrix $\hat{\boldsymbol{\Psi}}_{i}^{j}$ corresponding to the $i$ 'th focus quantity.

Remark 2.13. Two remarks related to Charkhi et al. (2015) are in place.

1. The weight vector $\hat{\boldsymbol{w}}$ is only uniquely determined when $\hat{\boldsymbol{\Psi}}^{j}$ is positive definite. As such, the bias subtraction may lead to non-unique weights.

2. Charkhi et al. (2015) consider a weighting scheme in which the weights sum to one but are not necessarily positive. Simulation results have shown that it is advisable to keep the positivity constraint in our autoregressive setup because weights can otherwise become large in magnitude and rather unstable.

Remark 2.14. Autoregressive models of infinite order have been considered by Berk (1974) and Lewis and Reinsel (1985), among others. It is an intriguing question whether the current framework can be extended to $V A R(\infty)$ models. We argue that the main difficulty is the estimation of the infinitely many local-to-zero parameters. Let us consider the univariate model $y_{T, t}=\beta y_{T, t-1}+\sum_{j=1}^{\infty}\left(\frac{\delta_{j}}{\sqrt{T}}\right) y_{T, t-1-j}$ $+u_{t}$ as an illustration. We conjecture ${ }^{10}$ that the asymptotic distribution of the approximating AR(1) model follows $\sqrt{T}(\hat{\beta}-\beta) \stackrel{d}{\longrightarrow} \mathrm{N}\left(\sum_{j=1}^{\infty} \delta_{j} \beta^{j}, 1-\beta^{2}\right)$. The bias contribution to the AMSE now depends on infinitely many $\delta_{j}$. Their estimation would require the lag order of the largest approximating model to grow with sample size. Our proof of Theorem 2.4 does not easily allow for such an extension since we currently rely on the finite dimension of the companion matrix. A full exploration of this topic is left for further research.

There is one final result that forces us to look at the case $l>1$. Practitioners are usually interested in the impulse responses for several horizons. Using a separate weight vector for every horizon may (1) create impulse responses that vary

\footnotetext{
${ }^{10}$ We can be more precise concerning our assumptions: Theorem 2.4 has shown that the asymptotic results are governed by the process with the local-to-zero parameters being set equal to zero. We assume that this remains true when there are infinitely many local-to-zero parameters.
} 


\section{CHAPTER 2}

irregularly from one horizon to the next due to strong changes in the weights, and (2) result in confidence intervals that do not take into account the dependence between the horizons. Theorem 2.15 below extends the result of Liu (2015) to obtain asymptotically valid confidence bands for several horizons.

Theorem 2.15 (Joint Confidence Bands). Under the Assumptions of Theorem 2.7, if $w_{m}(\hat{\boldsymbol{\delta}}) \stackrel{d}{\longrightarrow} w_{m}\left(\boldsymbol{R}_{\delta}\right)$, and if $\boldsymbol{D}_{\theta}\left(\boldsymbol{\Omega}^{-1} \otimes \boldsymbol{\Sigma}\right) \boldsymbol{D}_{\theta}^{\prime}+\boldsymbol{D}_{\sigma} \boldsymbol{\Xi}_{22} \boldsymbol{D}_{\sigma} \succ 0$, then

$$
\begin{aligned}
(\sqrt{T}(\overline{\boldsymbol{\mu}}(\hat{\boldsymbol{w}})- & \left.\left.\boldsymbol{\mu}\left(\boldsymbol{\theta}_{T, p}, \boldsymbol{\sigma}\right)\right)-\boldsymbol{D}_{\theta} \sum_{m=p_{1}}^{p} \hat{w}_{m} \hat{\boldsymbol{C}}_{m} \hat{\boldsymbol{\delta}}\right)^{\prime}\left(\boldsymbol{D}_{\theta}\left(\hat{\boldsymbol{\Omega}}^{-1} \otimes \hat{\boldsymbol{\Sigma}}\right) \boldsymbol{D}_{\theta}^{\prime}+\boldsymbol{D}_{\sigma} \hat{\boldsymbol{\Xi}}_{22} \boldsymbol{D}_{\sigma}\right)^{-1} \\
& \times\left(\sqrt{T}\left(\overline{\boldsymbol{\mu}}(\hat{\boldsymbol{w}})-\boldsymbol{\mu}\left(\boldsymbol{\theta}_{T, p}, \boldsymbol{\sigma}\right)\right)-\boldsymbol{D}_{\theta} \sum_{m=p_{1}}^{p} \hat{w}_{m} \hat{\boldsymbol{C}}_{m} \hat{\boldsymbol{\delta}}\right) \stackrel{d}{\longrightarrow} \chi_{l}^{2},
\end{aligned}
$$

where $\chi_{l}^{2}$ is a chi-squared distribution with $l$ degrees of freedom.

Remark 2.16. There is one practical concern which has not been addressed, namely the choices for $p_{1}$ and $p . p_{1}$ will turn out to be unimportant. To see this, we consider the expression for $\widehat{F I C}_{m}$ (a similar reasoning applies to the plug-in weights). The terms $\boldsymbol{D}_{\theta} \hat{\boldsymbol{P}}_{m}(\hat{\boldsymbol{\Omega}} \otimes \hat{\boldsymbol{\Sigma}}) \hat{\boldsymbol{P}}_{m} \boldsymbol{D}_{\theta}^{\prime}$ and $\boldsymbol{D}_{\sigma} \hat{\boldsymbol{\Xi}}_{22} \boldsymbol{D}_{\sigma}^{\prime}$ in Equation (2.9) do not depend on $p_{1}$. So it remains to inspect the contribution $\boldsymbol{D}_{\theta} \hat{\boldsymbol{C}}_{m}\left(\hat{\boldsymbol{\delta}} \hat{\boldsymbol{\delta}}^{\prime}-\boldsymbol{S}_{0}^{\prime} \hat{\boldsymbol{\Omega}}^{-1} \boldsymbol{S}_{0}\right.$ $\otimes \hat{\boldsymbol{\Sigma}}) \hat{\boldsymbol{C}}_{m}^{\prime} \boldsymbol{D}_{\theta}^{\prime}$. Using $\hat{\boldsymbol{\delta}}=\left(\boldsymbol{S}_{0}^{\prime} \otimes \boldsymbol{I}_{K}\right) \sqrt{T} \hat{\boldsymbol{\theta}}_{T, p}$ we can rewrite this contribution as

$$
\boldsymbol{D}_{\theta}\left(\hat{\boldsymbol{C}_{m}}\left(\boldsymbol{S}_{0}^{\prime} \otimes \boldsymbol{I}_{K}\right)\right)\left[\left(\sqrt{T} \hat{\boldsymbol{\theta}}_{T, p}\right)\left(\sqrt{T} \hat{\boldsymbol{\theta}}_{T, p}\right)^{\prime}-\hat{\boldsymbol{\Omega}}^{-1} \otimes \hat{\boldsymbol{\Sigma}}\right]\left(\hat{\boldsymbol{C}}_{m}\left(\boldsymbol{S}_{0}^{\prime} \otimes \boldsymbol{I}_{K}\right)\right)^{\prime} \boldsymbol{D}_{\theta}^{\prime} .
$$

By definition of $\hat{\boldsymbol{C}}_{m}$, we have

$$
\begin{aligned}
\hat{\boldsymbol{C}}_{m}\left(\boldsymbol{S}_{0}^{\prime} \otimes \boldsymbol{I}_{K}\right) & =\left(\boldsymbol{S}_{m}\left[\boldsymbol{S}_{m}^{\prime} \hat{\boldsymbol{\Omega}} \boldsymbol{S}_{m}\right]^{-1} \boldsymbol{S}_{m}^{\prime} \hat{\boldsymbol{\Omega}}-\boldsymbol{I}_{K p}\right)\left[\boldsymbol{S}_{0}\left(\boldsymbol{I}_{K p_{2}}-\boldsymbol{\Pi}_{m}^{\prime} \boldsymbol{\Pi}_{m}\right) \boldsymbol{S}_{0}^{\prime}\right] \otimes \boldsymbol{I}_{K} \\
& =\left(\boldsymbol{S}_{m}\left[\boldsymbol{S}_{m}^{\prime} \hat{\boldsymbol{\Omega}} \boldsymbol{S}_{m}\right]^{-1} \boldsymbol{S}_{m}^{\prime} \hat{\boldsymbol{\Omega}}-\boldsymbol{I}_{K p}\right) \underbrace{\left.\left(\begin{array}{cc}
\mathbf{O} & \mathbf{O} \\
\mathbf{O} & \boldsymbol{I}_{K(p-m)}
\end{array}\right] \otimes \boldsymbol{I}_{K}\right)}_{\mathcal{B}},
\end{aligned}
$$

thereby showing that actually none of the contributions to $\widehat{F I C}_{m}$ depends on $p_{1}$. However, the zero pattern of the matrix $\mathcal{B}$ in Equation (2.12) will only cause a non-diverging value for $\widehat{F I C}_{m}$ if models are chosen such that $m \in \mathcal{M}=\left\{p_{1}, p_{1}+\right.$ $1, \ldots, p\}$. This supports the claim in Remark 2.5. The lag order of the full model, that is $p$, might be chosen by AIC or set equal to an a priori selected $p_{\max }$.

\subsubsection{The Effects of Inconsistently Estimating Delta}

Equation (2.8) showed that $\hat{\boldsymbol{\delta}}$ converges to a normally distributed random vector centered around $\boldsymbol{\delta}$. How does this influence the selection and averaging procedures? Clearly, $\widehat{F I C}_{m}, \hat{\Psi}_{m, l}^{\text {Biased }}$, and $\hat{\Psi}_{m, l}^{\text {Bias cor }}$ will not converge in probability to the AMSE 
they are intended to estimate. The limiting distributions of these quantities are highlighted in Theorems 2.17 and 2.18. The plug-in results are stated for $\hat{\Psi}_{m, l}^{\text {Bias cor }}$, but a simple omission of the bias correction term would give the corresponding findings for $\hat{\Psi}_{m, l}^{\text {Biased }}$.

\section{Theorem 2.17. The Asymptotic Behavior of $\widehat{F I C}_{m}$.}

Under the Assumptions of Theorem 2.7 we have for $m \in \mathcal{M} \backslash p$,

$$
\begin{array}{r}
\widehat{F I C}_{m} \stackrel{d}{\longrightarrow} \boldsymbol{D}_{\theta}\left[\boldsymbol{C}_{m}\left(\boldsymbol{R}_{\delta} \boldsymbol{R}_{\delta}^{\prime}-\left(\boldsymbol{S}_{0}^{\prime} \boldsymbol{\Omega}^{-1} \boldsymbol{S}_{0} \otimes \boldsymbol{\Sigma}\right)\right) \boldsymbol{C}_{m}^{\prime}+\boldsymbol{P}_{m}(\boldsymbol{\Omega} \otimes \boldsymbol{\Sigma}) \boldsymbol{P}_{m}\right] \boldsymbol{D}_{\theta}^{\prime} \\
+\boldsymbol{D}_{\sigma} \boldsymbol{\Xi}_{22} \boldsymbol{D}_{\sigma}^{\prime}:=F I C_{m}^{\infty} \\
\sim a_{m} \chi_{\text {noncentral }}^{2}\left(1,\left(\boldsymbol{D}_{\theta} \boldsymbol{C}_{m} \boldsymbol{\delta}\right)^{2} / a_{m}\right)-a_{m}+\boldsymbol{D}_{\theta} \boldsymbol{P}_{m}(\boldsymbol{\Omega} \otimes \boldsymbol{\Sigma}) \boldsymbol{P}_{m} \boldsymbol{D}_{\theta}^{\prime}+\boldsymbol{D}_{\sigma} \boldsymbol{\Xi}_{22} \boldsymbol{D}_{\sigma}^{\prime},
\end{array}
$$

where $a_{m}=\boldsymbol{D}_{\theta} \boldsymbol{C}_{m}\left(\boldsymbol{S}_{0}^{\prime} \boldsymbol{\Omega}^{-1} \boldsymbol{S}_{0} \otimes \boldsymbol{\Sigma}\right) \boldsymbol{C}_{m}^{\prime} \boldsymbol{D}_{\theta}^{\prime}$, and $\chi_{\text {noncentral }}^{2}(1, \lambda)$ denotes a noncentral chi-squared distributed random variable with 1 degree of freedom and noncentrality parameter $\lambda$. It can be shown that $\mathrm{E}\left(F I C_{m}^{\infty}\right)=A M S E\left(\mu\left(\hat{\boldsymbol{\theta}}_{T, m}, \mathbf{0}_{K^{2}(p-m)}\right.\right.$, $\hat{\boldsymbol{\sigma}}))$ and $\operatorname{var}\left(F I C_{m}^{\infty}\right)=2 a_{m}\left(a_{m}+2\left(\boldsymbol{D}_{\theta} \boldsymbol{C}_{m} \boldsymbol{\delta}\right)^{2}\right)$. For the full model, $m=p$, we have

$$
\widehat{F I C}_{p} \stackrel{p}{\longrightarrow} F I C_{p}^{\infty}=\boldsymbol{D}_{\theta}\left(\boldsymbol{\Omega}^{-1} \otimes \boldsymbol{\Sigma}\right) \boldsymbol{D}_{\theta}^{\prime}+\boldsymbol{D}_{\sigma} \boldsymbol{\Xi}_{22} \boldsymbol{D}_{\sigma}^{\prime}=A M S E\left(\mu\left(\hat{\boldsymbol{\theta}}_{T, p}, \hat{\boldsymbol{\sigma}}\right)\right) .
$$

Theorem 2.18. The Asymptotic Behavior of $\hat{\Psi}_{m, l}^{\text {Biased }}$ and $\hat{\Psi}_{m, l}^{\text {Bias cor }}$. Under the Assumptions of Theorem 2.7 we have for $m, l \in \mathcal{M} \backslash p$,

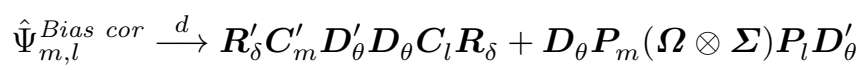

$$
\begin{aligned}
& -\boldsymbol{D}_{\theta} \boldsymbol{C}_{m}\left(\boldsymbol{S}_{0}^{\prime} \boldsymbol{\Omega}^{-1} \boldsymbol{S}_{0} \otimes \boldsymbol{\Sigma}\right) \boldsymbol{C}_{m}^{\prime} \boldsymbol{D}_{\theta}^{\prime}:=\boldsymbol{\Psi}_{m, l}^{\text {Bias cor }, \infty} .
\end{aligned}
$$

Two cases can be distinguished:

(a) If $m=l$, then $\boldsymbol{\Psi}_{m, m}^{\text {Bias cor, } \infty} \sim a_{m} \chi_{\text {noncentral }}^{2}\left(1,\left(\boldsymbol{D}_{\theta} \boldsymbol{C}_{m} \boldsymbol{\delta}\right)^{2} / a_{m}\right)-a_{m}$ $+\boldsymbol{D}_{\theta} \boldsymbol{P}_{m}(\boldsymbol{\Omega} \otimes \boldsymbol{\Sigma}) \boldsymbol{P}_{m} \boldsymbol{D}_{\theta}^{\prime}$.

(b) Define $\mathcal{A}=\boldsymbol{S}_{0}^{\prime} \boldsymbol{\Omega}^{-1} \boldsymbol{S}_{0} \otimes \boldsymbol{\Sigma}$, and consider the eigenvalue decomposition $\frac{1}{2} \mathcal{A}^{1 / 2}\left(\boldsymbol{C}_{m}^{\prime} \boldsymbol{D}_{\theta} \boldsymbol{D}_{\theta}^{\prime} \boldsymbol{C}_{l}+\boldsymbol{C}_{l}^{\prime} \boldsymbol{D}_{\theta} \boldsymbol{D}_{\theta}^{\prime} \boldsymbol{C}_{m}\right) \mathcal{A}^{1 / 2}=\sum_{i=1}^{2} \lambda_{i} \boldsymbol{v}_{i} \boldsymbol{v}_{i}^{\prime}$, where $\lambda_{i}$ denotes the eigenvalue corresponding to the eigenvector $\boldsymbol{v}_{i}$. If $m \neq l$, then

$$
\begin{aligned}
\boldsymbol{\Psi}_{m, l}^{\text {Bias cor }, \infty} \sim \sum_{i=1}^{2} \lambda_{i} \chi_{\text {noncentral }}^{2} & \left(1,\left(\boldsymbol{v}_{i}^{\prime} \mathcal{A}^{-1 / 2} \boldsymbol{\delta}\right)^{2}\right)+\boldsymbol{D}_{\theta} \boldsymbol{P}_{m}(\boldsymbol{\Omega} \otimes \boldsymbol{\Sigma}) \boldsymbol{P}_{l} \boldsymbol{D}_{\theta}^{\prime} \\
& -\boldsymbol{D}_{\theta} \boldsymbol{C}_{m}\left(\boldsymbol{S}_{0}^{\prime} \boldsymbol{\Omega}^{-1} \boldsymbol{S}_{0} \otimes \boldsymbol{\Sigma}\right) \boldsymbol{C}_{m}^{\prime} \boldsymbol{D}_{\theta}^{\prime} .
\end{aligned}
$$

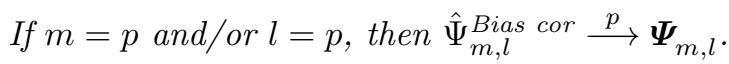

Theorems 2.17 and 2.18 state the limiting distribution of the FIC and the matrix elements that enter the weighting scheme. Based on the random limits of these quantities, we might expect that our methods will not truly minimize the AMSE among either model choices or model weights. We proceed with a small illustration to stress the difference between knowing $\boldsymbol{\delta}$ and having an estimator $\hat{\boldsymbol{\delta}}$ that converges in distribution only. 


\section{An Illustration}

Consider the simplified DGP $y_{T, t}=0.5 y_{T, t-1}+\frac{\delta}{\sqrt{T}} y_{T, t-2}+u_{t}$, with $\operatorname{var}\left(u_{t}\right)=$ $\sigma^{2}=1$, and a focus on the impulse response at horizon 1 (i.e. $\boldsymbol{D}_{\theta}=(1,0)$ and $\left.\boldsymbol{D}_{\sigma}=0\right)$. The model set is $\mathcal{M}=\{1,2\}$. This simplified setting makes the asymptotic behavior of the FIC and plug-in weights analytically tractable. Figure 2.1(a) depicts $F I C_{1}^{\infty}$ and $F I C_{2}^{\infty}$ as a function of $\delta$. Note that $F I C_{1}^{\infty}$ converges in distribution and has a nonzero probability to give an outcome below $F I C_{2}^{\infty}$. This asymptotic selection probability of the model with $m=1$ can be calculated analytically using Theorem 2.17. Figure 2.1(b) shows that the FIC does not select the model with the smallest AMSE with probability one.

Our simplified model can also be used to examine the effect of $\hat{\delta}$ on the plug-in weights. We focus on the weights in the absence of bias correction. ${ }^{11}$ The $(2 \times 2)$ limiting matrix $\boldsymbol{\Psi}^{\infty}$ is

$$
\boldsymbol{\Psi}^{\infty}=\left[\begin{array}{cc}
a_{1} \chi_{\text {noncentral }}^{2}\left(1,\left(\boldsymbol{D}_{\theta} \boldsymbol{C}_{1} \boldsymbol{\delta}\right)^{2} / a_{1}\right)+\sigma^{2} \boldsymbol{D}_{\theta} \boldsymbol{P}_{1} \boldsymbol{D}_{\theta}^{\prime} & \sigma^{2} \boldsymbol{D}_{\theta} \boldsymbol{P}_{1} \boldsymbol{D}_{\theta}^{\prime} \\
\sigma^{2} \boldsymbol{D}_{\theta} \boldsymbol{P}_{1} \boldsymbol{D}_{\theta}^{\prime} & \sigma^{2} \boldsymbol{D}_{\theta} \boldsymbol{\Omega}^{-1} \boldsymbol{D}_{\theta}^{\prime}
\end{array}\right],
$$

where $a_{1}=\sigma^{2} \boldsymbol{D}_{\theta} \boldsymbol{C}_{1} \boldsymbol{S}_{0}^{\prime} \boldsymbol{\Omega} \boldsymbol{S}_{0} \boldsymbol{C}_{1}^{\prime} \boldsymbol{D}_{\theta}^{\prime}$ (see Theorem 2.18). Let $w^{*}$ denote the asymptotically optimal plug-in weight for the model with $m=1$. We have

$$
\operatorname{Pr}\left(w^{*} \leq x\right)=\operatorname{Pr}\left(\chi_{\text {noncentral }}^{2}\left(1,\left(\boldsymbol{D}_{\theta} \boldsymbol{C}_{1} \boldsymbol{\delta}\right)^{2} / a_{1}\right) \geq \frac{\sigma^{2} \boldsymbol{D}_{\theta}\left(\boldsymbol{\Omega}^{-1}-\boldsymbol{P}_{1}\right) \boldsymbol{D}_{\theta}^{\prime}[1-x]}{a_{1} x}\right) .
$$

Figure 2.2 shows the area between the $5 \%$ and $95 \%$ quantiles of $w^{*}$ together with the optimal weight for known delta. We see that the asymptotic distribution of $w^{*}$ is located closer to zero than the optimal infeasible weights. This is unsurprising because the lack of bias-correction causes (on average) an overestimation of the AMSE of the model with $m=1$.

Remark 2.19. The exposition in this section was based on a simplified model. We concluded that the absence of a consistent estimator for $\delta$ translates into suboptimal model selection and suboptimal model averaging. Also in more elaborate models the FIC and the elements of the weighting matrix $\boldsymbol{\Psi}$ will converge to random variables (except for $m=p$ ). It is key to realize that the AMSE with estimated $\boldsymbol{\delta}$ will not coincide with the AMSE that can be attained if $\boldsymbol{\delta}$ was either known or consistently estimated. We conjecture that these considerations are equally relevant outside an autoregressive framework, e.g. in the regression framework discussed in Liu (2015) and the likelihood framework of Charkhi et al. (2015).

\subsection{Simulations}

This simulation section consists of three parts. In the first part we will verify our derivations for the simplified DGP, and see how the suboptimal selection/averaging

\footnotetext{
${ }^{11}$ There is a finite probability for the matrix $\boldsymbol{\Phi}^{\infty}$ to have a negative eigenvalue when the bias correction is applied. This severely complicates the derivations, so we exclude this case from our analysis. For the $\boldsymbol{\Psi}^{\infty}$ matrix without bias correction we will have $\boldsymbol{\Psi}^{\infty} \succ 0$ if $\boldsymbol{D}_{\theta}^{\prime}\left(\boldsymbol{\Omega}^{-1}-\boldsymbol{P}_{1}\right) \boldsymbol{D}_{\theta}>$ 0 . The latter requirement is equivalent to $\boldsymbol{D}_{\theta}^{\prime}\left[\begin{array}{c}-\omega_{12} \\ \omega_{11}\end{array}\right] \neq 0$.
} 


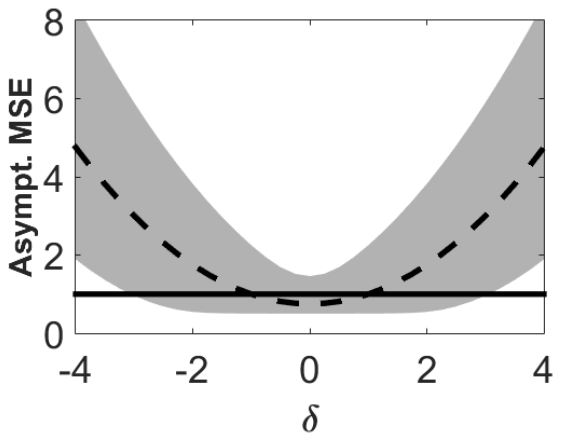

(a)

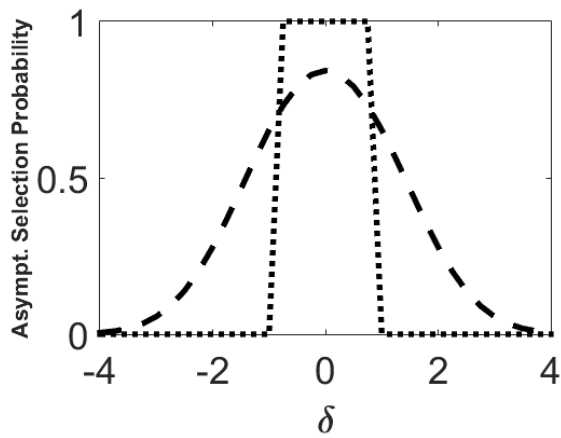

(b)

Figure 2.1: (a) The asymptotic MSE of the models with one and two lags (black broken and solid line, respectively). The area between the $5 \%$ and $95 \%$ quantiles of $F I C_{1}^{\infty}$ is shaded gray. $\widehat{F I C}_{2}$ converges in probability to the values of the black line. (b) The asymptotic selection probabilities of the model with $m=1$. The infeasible estimator (dotted line) takes a binary decision based on whether the solid or broken line in graph (a) is lowest. Model selection based on the Focused Information Criterion results in a smoothed asymptotic selection probability because $\widehat{F I C}_{1}$ converges in distribution.

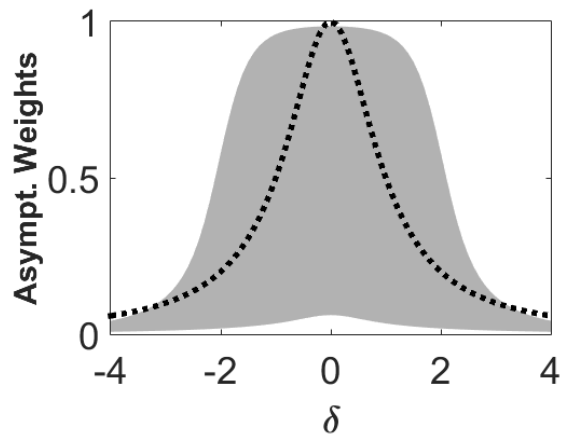

Figure 2.2: The $5 \%$ and $95 \%$ quantiles of the asymptotic distribution of the weights as a shaded gray area, see Equation (2.13). The dotted line describes the asymptotically optimal weights which can only be obtained if either $\delta$ is known (infeasible) or a consistent estimator for $\delta$ is available.

affects the finite sample MSE. This section is followed by a study of the impulse responses for different horizons in a univariate and multivariate setting. All our 


\section{CHAPTER 2}

graphs are made as a function of the scalar $\delta$. This scalar measures the amount of misspecification and the closeness to unit root. ${ }^{12}$ Any missing starting values in the autoregressive recursion were replaced by zeros, and the first 100 data points were omitted as a presample. All results are based on 100,000 Monte Carlo replications.

The performance of the various methods was assessed using the empirical mean squared error. For model selection the featured methods are:

1. The Akaike information criterion ('AIC') and Bayesian information criterion ('BIC'), cf. e.g. section 4.3 of Lütkepohl (2005) and the original papers by Akaike (1998) and Schwarz (1978).

2. The 'FIC' from Equation (2.9) with estimated $\boldsymbol{\delta}$.

3. An infeasible version of the FIC abbreviated as 'Infeas'. This information criterion is based on population quantities, especially $\boldsymbol{\delta}$ is known.

For the model averaging setup we consider:

1. 'sAIC' and 'sBIC' as smoothed counterparts of the AIC and BIC, see Burnham and Anderson (2002). To illustrate, let $A I C(m)$ denote the AIC for model $m \in \mathcal{M}$. The smoothed AIC weight of model $m$ is $\exp \left(-\frac{1}{2} A I C(m)\right) / \sum_{m \in \mathcal{M}} \exp \left(-\frac{1}{2} A I C(m)\right)$.

2. Three plug-in averages are reported. 'Plug-in' and 'Plug-in Corr.' are computed from Equations (2.10) and (2.11), where only the second average uses the bias correction on $\hat{\boldsymbol{\delta}} \hat{\boldsymbol{\delta}}^{\prime}$. The plug-in average based on known $\boldsymbol{\delta}$ is denoted 'Infeas'.

3. The 'Jackknife' model averaging procedure detailed in Hansen and Racine (2012) and Zhang et al. (2013).

4. The Stein combination shrinkage method used in the simulation section of Hansen (2016) is abbreviated 'SteinH'. This shrinkage method combines $\operatorname{VAR}(1)$ through $\operatorname{VAR}(p)$ models as well as univariate $\operatorname{AR}(1)$ through $\operatorname{AR}(p)$ models. Our DGP contains considerable interaction between the crosssectional units so we also consider a shrinkage method abbreviated 'Stein' which only combines the $\operatorname{VAR}(1)$ through $\operatorname{VAR}(p)$.

\subsubsection{The Simplified DGP}

Figures 2.3 and 2.4 provide the finite sample confirmation of the intuition we gained from the simplified DGP. ${ }^{13}$ The wide spread in the empirical distribution of $\widehat{F I C}_{1}$ shown in Figure 2.3(a) results indeed in a smoothed instead of binary

\footnotetext{
${ }^{12}$ Previous studies (e.g. Hansen (2007), Hansen (2008), Hansen and Racine (2012), Liu and Okui (2013), Zhang et al. (2013) and Liu (2015)) show the performance as a function of the population $R^{2}$. This representation is inconvenient in our dynamic setup because it is unclear when the boundary of the stationarity region is approached.

${ }^{13}$ In this section we have rescaled the empirical MSE by the sample size to make it comparable to the asymptotic results of Figures 2.1 and 2.2, hence the label empirical asymptotic MSE.
} 
selection between the models (see Figure 2.3(b)). The performance of the feasible FIC is therefore worse than that of the infeasible FIC that assumes $\delta$ to be known. At high $|\delta|$, we see that the probability to select the wrong model is small. The feasible FIC therefore performs similarly to its infeasible counterpart for large amounts of misspecification only.

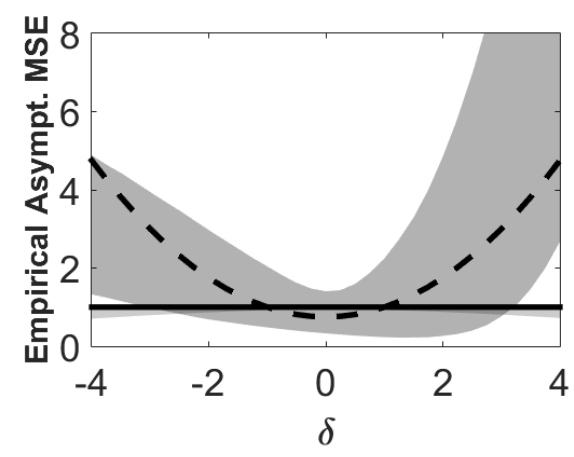

(a)

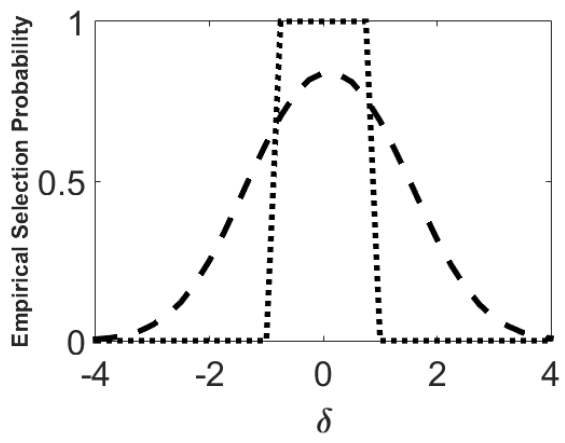

(b)

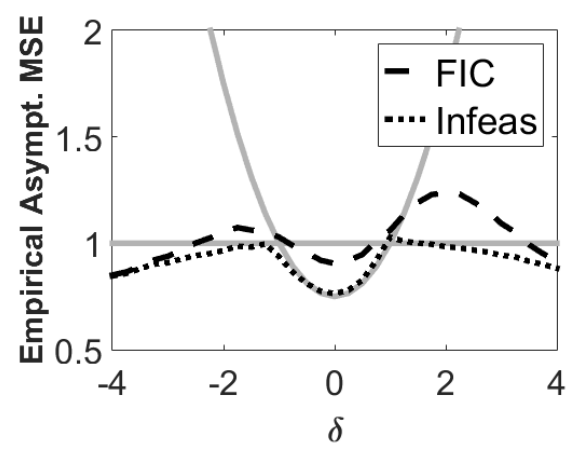

(c)

Figure 2.3: (a) The empirical asymptotic MSE of the models with one and two lags (black broken and solid line, respectively). The area between the $5 \%$ and $95 \%$ empirical quantiles of $\widehat{F I C}_{1}$ and $\widehat{F I C}_{2}$ are shaded in dark (larger area) and light gray. (b) The empirical selection probabilities of the FIC. (c) The AMSE of the models with $m=1$ and $m=2$ together with the empirical MSE of the feasible FIC (broken) and infeasible FIC (dotted). This figure was obtained for $T=100$ and should be compared with the asymptotic results in Figure 2.1.

In Figure 2.4, the three panels display results on the plug-in averages. The quantiles of the weight distribution without bias correction should be compared to those in Equation (2.13) and Figure 2.2. The results match. Figure 2.4(b) shows the quantiles of the weight distribution with bias correction. As expected, 


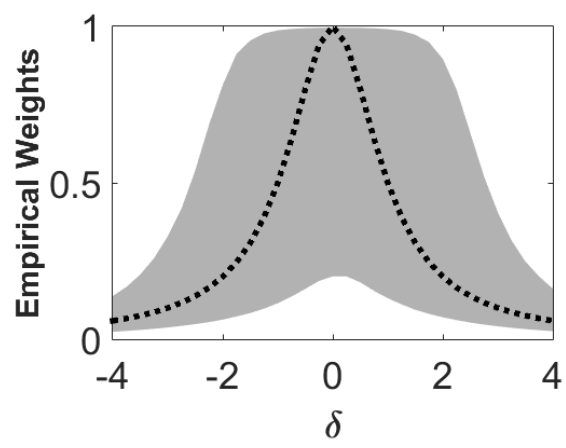

(a)

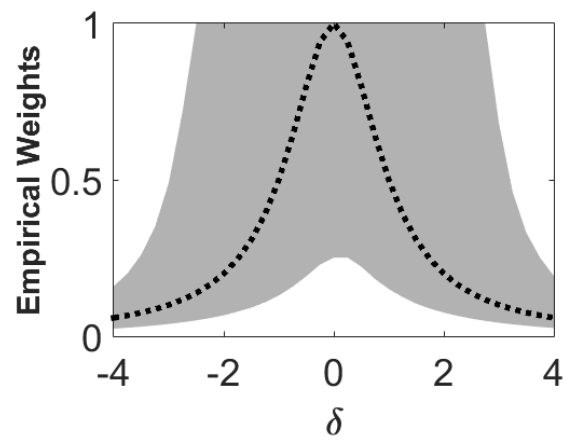

(b)

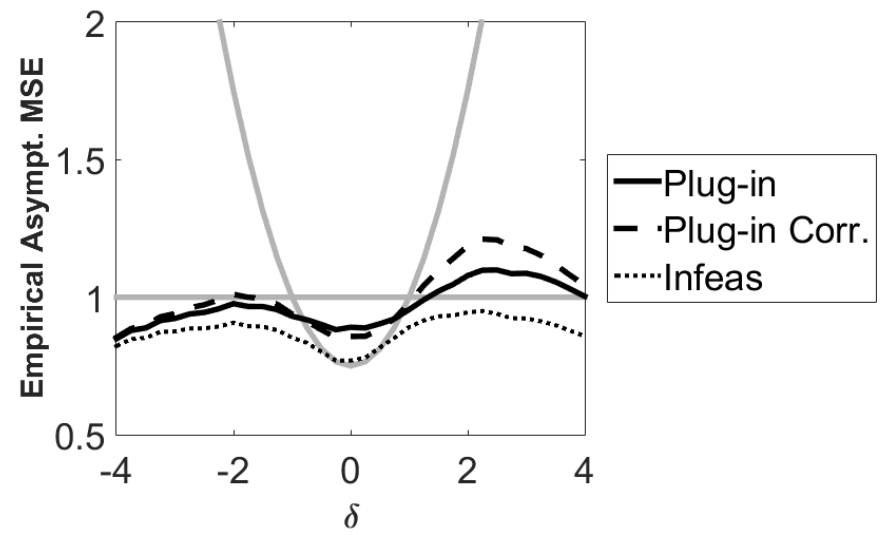

(c)

Figure 2.4: The $5 \%$ and $95 \%$ empirical quantiles of the weights distribution without bias correction (a) and with bias correction (b). The infeasible weights are represented by the dotted line. The empirical MSE of plug-in methods is shown in (c). The sample size is $T=100$.

this distribution is shifted towards higher weights because the upward bias of the AMSE of the model with $m=1$ is removed. We can see in 2.4(c) that the plug-in averages do not perform as well as the infeasible estimator. Unreported simulation results at a sample size of $T=1000$ confirm that this effect does not disappear with sample size. The inconsistent estimation of $\delta$ again causes the feasible weights to differ from optimal weights. 


\subsubsection{Simulation Results for an Autoregressive Model}

Further simulations are based on the following model. ${ }^{14}$

$$
y_{T, t}=0.5 y_{T, t-1}+\frac{\delta}{\sqrt{T}} y_{T, t-2}+\frac{\delta}{2 \sqrt{T}} y_{T, t-3}+u_{t}, \quad u_{t} \stackrel{i . i . d .}{\sim} \mathcal{N}(0,1) .
$$

The second and third lag are local-to-zero implying that $\mathcal{M}=\{1,2,3\}$. The coefficients in front of the misspecified lags decline linearly as in Liu (2015), where $\delta$ governs the amount of misspecification. The largest modulus eigenvalue of the companion matrix is about 0.3 at $\delta=-0.2$ and increases monotonously to approximately 0.9 at the boundaries of the interval $[-4,2]$.

Remark 2.20. The AMSEs of the impulse response at horizon 1 are the same for $m=2$ and $m=3$. The plug-in weights are not unique, also see Remark 2.13.

\section{MSE Comparison}

The empirical MSE of the various selection methods are shown in Figure 2.5 for the impulse responses at horizon 1,3 , and 5 . Due to the strong penalty on model complexity, the BIC performs well for small amounts of misspecification, but its performance quickly deteriorates as $|\delta|$ increases. The performance of the AIC and the feasible version of the FIC are comparable for large areas of the parameter space, with neither of these methods being preferred to the other. The infeasible FIC is very frequently the preferred method.

Model averaging results are reported in Figure 2.6. The behavior of the smoothed BIC procedure is similar to that of its selection counterpart, i.e. it only performs well for small $\delta$. The same remark applies to the plug-in average with bias correction. The Jackknife, smoothed AIC, and the plug-in average without bias correction are close competitors, where the plug-in average is a better candidate for large $|\delta|$. The performance of the plug-in average with known $\delta$ is best. It even performs uniformly best at the larger sample size of $T=1000$.

What causes the superior performance of the infeasible estimators? Our simulation findings can be understood from the intuition that was gained from the simplified DGP. Panel (a) and (b) from Figure 2.7 show the empirical MSE of the three models, $m \in\{1,2,3\}$, together with the AMSE of these models. The asymptotic approximation is close for $T=100$ and improves further at $T=1000$. The selection probabilities in panels (c) and (d) reveal how the infeasible estimator takes a binary decision with the lag length increasing with $\delta$. For the simplified DGP we have seen how the convergence in distribution of $\hat{\delta}$ causes smeared out selection probabilities instead of the binary decision. This effect is also observed in the graphs, even at the large sample size of $T=1000$. The panels (e) and (f) tell the same story for the plug-in weights.

\footnotetext{
${ }^{14}$ We show in Section 2.A.1 in the appendix that the simplified model with $p_{1}=p_{2}=1$, i.e. $y_{T, t}=\beta y_{T, t-1}+\frac{\delta}{\sqrt{T}} y_{T, t-2}+u_{t}$, is special because the gradient vector has no influence on model selection and plug-in averaging. We extend the model with an additional lag to see the influence of the impulse response horizon.
} 


\section{CHAPTER 2}

We also performed unreported simulations where we focused on several impulse responses simultaneously, see Remarks 2.10 and 2.12. The trace is used to map the AMSE matrix to a scalar. The simulation outcomes were qualitatively similar to our results for the impulse responses at a single horizon.

\section{Confidence Intervals}

Confidence intervals/bands can be calculated based on Theorem 2.15. Simulation results are provided in Tables 2.1 and 2.2. The desired nominal coverage level is $90 \%$. Table 2.1 shows that the empirical coverage of the individual confidence intervals is consistently too low. At horizons 2 and 3 this under-coverage is least severe and decreases with sample size. The coverage of the confidence level for horizon 6 varies strongly across $\delta$ and can be very low. It is wellestablished in the literature (e.g. Kilian (1998b) and Kilian (2001)) that inference on impulse responses at higher horizons is inherently more difficult because of the increased nonlinearity in the parameters. This nonlinearity causes the delta method approximation to perform poorly. Figure 2.8 shows the histograms of $\sqrt{T}\left(\overline{\boldsymbol{\mu}}(\hat{\boldsymbol{w}})-\boldsymbol{\mu}\left(\boldsymbol{\theta}_{T}, \boldsymbol{\sigma}\right)\right)-\boldsymbol{D}_{\theta} \sum_{m=p_{1}}^{p_{1}+p_{2}} \hat{w}_{m} \hat{\boldsymbol{C}}_{m} \hat{\boldsymbol{\delta}}$ for the impulse responses at horizons 2 and 6 (corresponding to the boxed numbers in Table 2.1). Note that the confidence intervals/bands defined in Theorem 2.15 are based on the asymptotic normality of this expression. The sometimes severe under-coverage at horizon 6 should therefore not come as a surprise. This poor asymptotic approximation at horizon 6 also influences the empirical coverage of the confidence bands as can be seen in Table 2.2 .

\subsubsection{Simulation Results for a Vector Autoregressive Model}

Our simulation results are based on a bivariate VAR with DGP

$$
\begin{gathered}
\boldsymbol{y}_{T, t}=\left(\begin{array}{cc}
0.5 & 0 \\
0.5 & 0.5
\end{array}\right) \boldsymbol{y}_{T, t-1}+\frac{\delta}{\sqrt{T}}\left(\begin{array}{cc}
1 & 0 \\
0.5 & 1
\end{array}\right) \boldsymbol{y}_{T, t-2}+\frac{\delta}{2 \sqrt{T}}\left(\begin{array}{cc}
1 & 0 \\
0.5 & 1
\end{array}\right) \boldsymbol{y}_{T, t-3}+\boldsymbol{u}_{t}, \\
\boldsymbol{u}_{t} \stackrel{i . i . d .}{\sim} \mathcal{N}(\mathbf{0}, \boldsymbol{\Sigma}), \text { where } \boldsymbol{\Sigma}=\left(\begin{array}{cc}
1 & 0.17 \\
0.17 & 0.33
\end{array}\right),
\end{gathered}
$$

which is similar to the VAR used in Lütkepohl et al. (2015) for impulse response analysis. This process has the same roots as the univariate process of Equation (2.14) but with double multiplicity. The parameter $\delta$ governs the degree of misspecification, and the value of $\boldsymbol{\delta}$. For brevity, we only report MSE results of the response of variable 1 to a structural shock in variable 1 . Figures 2.9, and 2.10 show the results for horizons 2 and 6 . Similarly to the univariate results, none of the methods performs uniformly best. Only the infeasible methods get close to dominating all other methods for the large sample size of $T=1000$. The ragged spike for 'Infeas' in Figure 2.9(a) is caused by an abrupt binary decision to switch between models with different lag lengths. Finally, it is interesting to note that the Stein shrinkage methods perform well in comparison to the plug-in averaging procedure. 


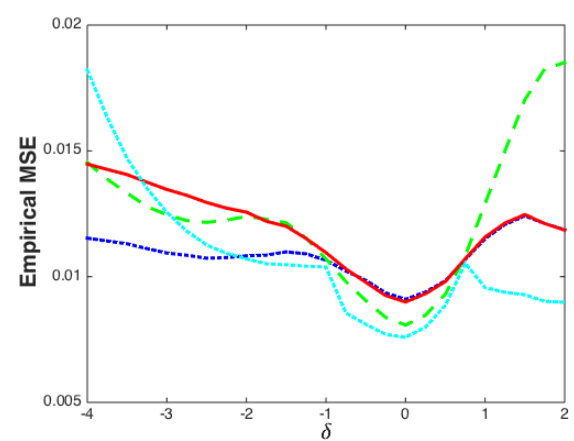

(a) $h=1, T=100$.

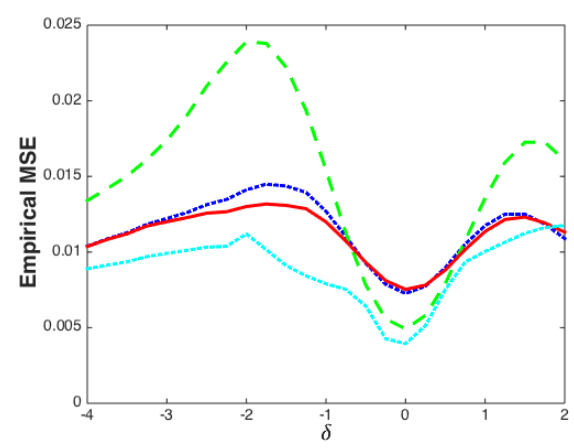

(c) $h=3, T=100$.

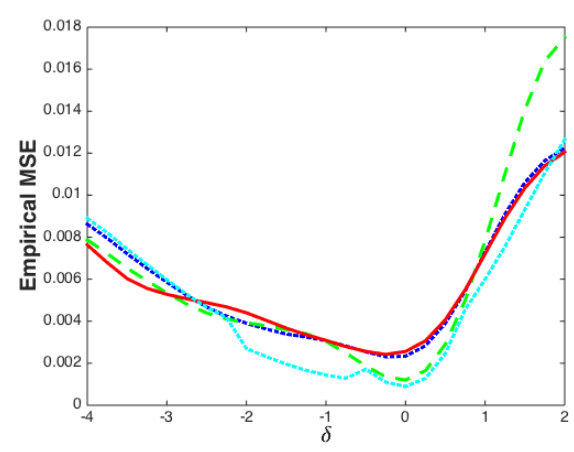

(e) $h=5, T=100$.

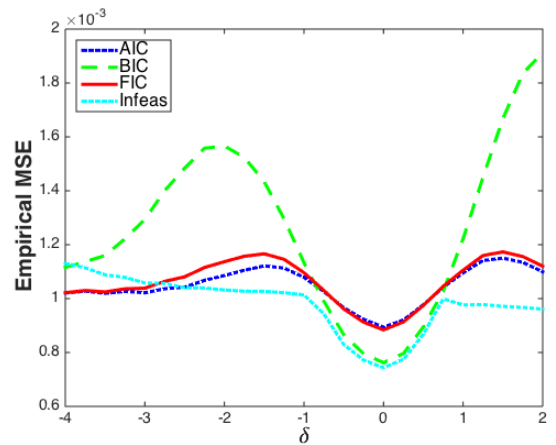

(b) $h=1, T=1000$.

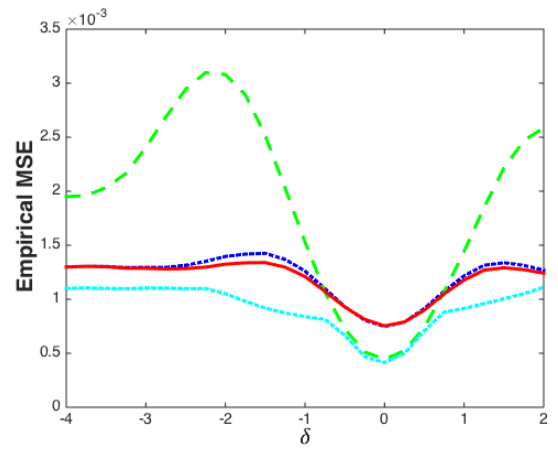

(d) $h=3, T=1000$.

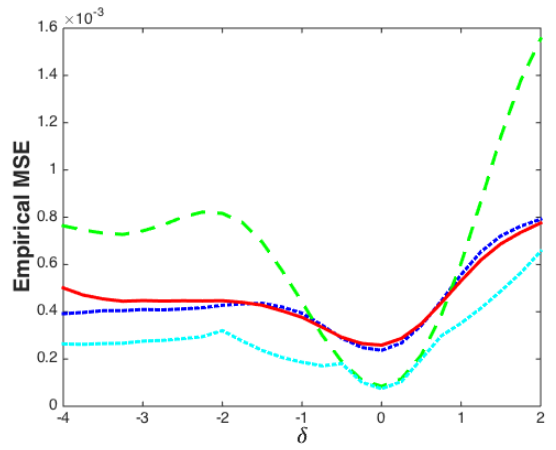

(f) $h=5, T=1000$.

Figure 2.5: The empirical MSE for model selection. The DGP is $y_{T, t}=$ $0.5 y_{T, t-1}+\frac{\delta}{\sqrt{T}} y_{T, t-2}+\frac{\delta}{2 \sqrt{T}} y_{T, t-3}+u_{t}$. 


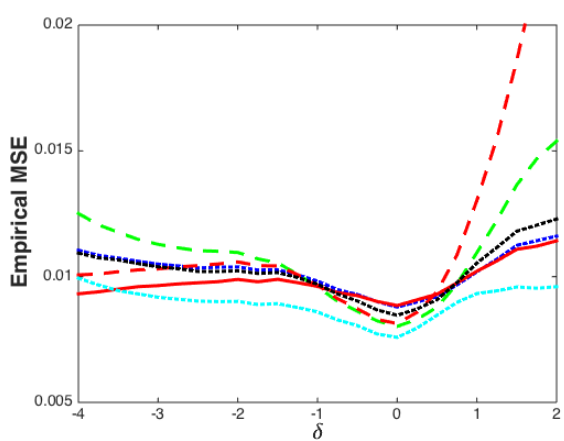

(a) $h=1, T=100$.

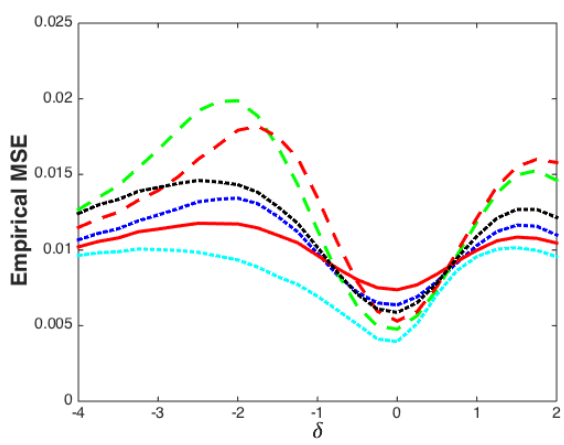

(c) $h=3, T=100$.

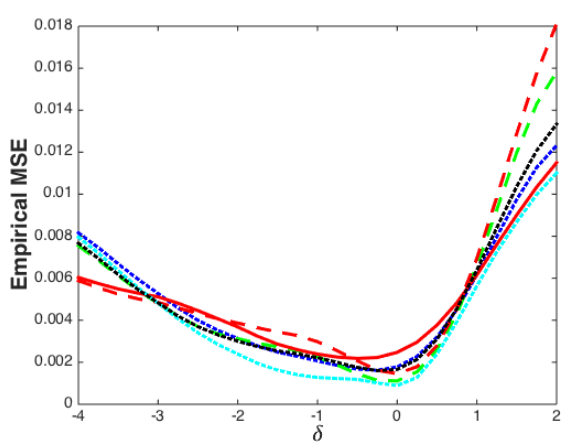

(e) $h=5, T=100$.

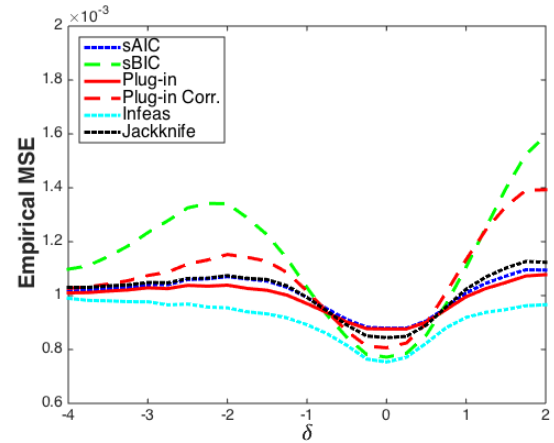

(b) $h=1, T=1000$.

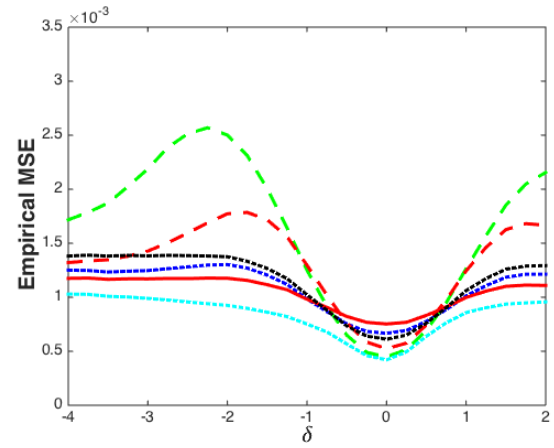

(d) $h=3, T=1000$.

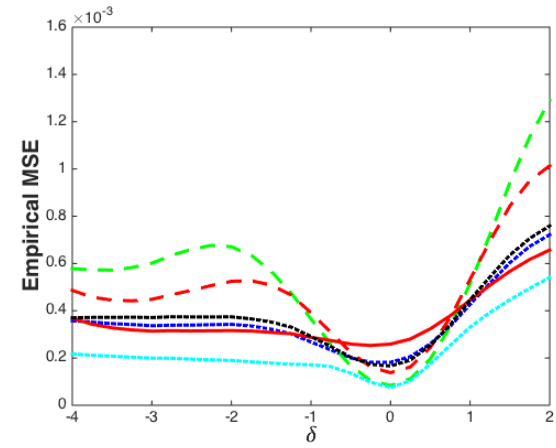

(f) $h=5, T=1000$.

Figure 2.6: The empirical MSE for model averaging. The DGP is $y_{T, t}=$ $0.5 y_{T, t-1}+\frac{\delta}{\sqrt{T}} y_{T, t-2}+\frac{\delta}{2 \sqrt{T}} y_{T, t-3}+u_{t}$. 


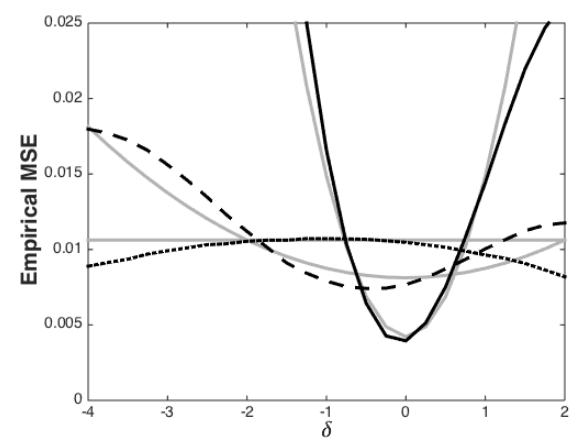

(a) $h=3, T=100$.
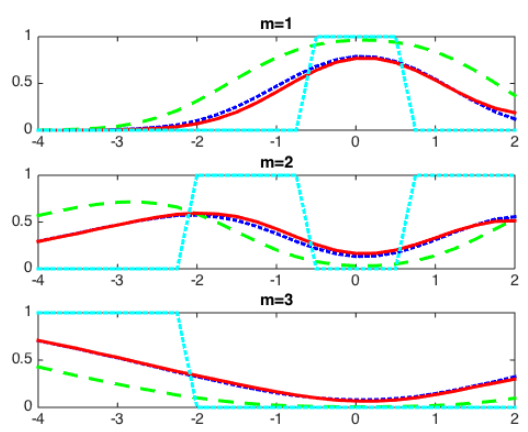

(c) $h=3, T=100$.
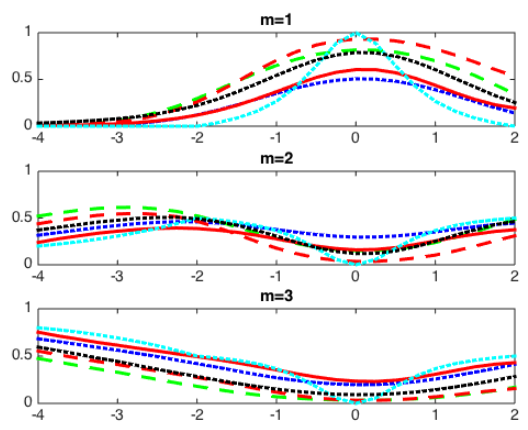

(e) $h=3, T=100$.

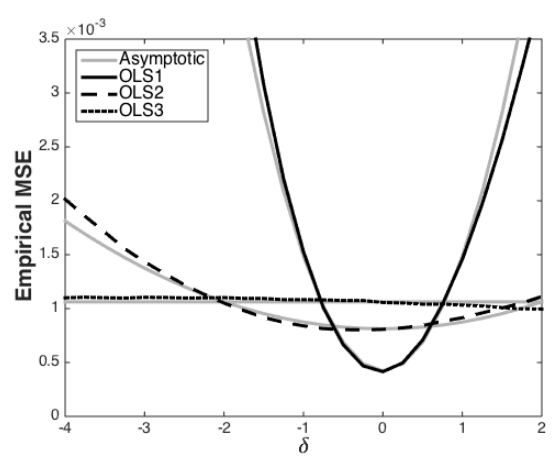

(b) $h=3, T=1000$.
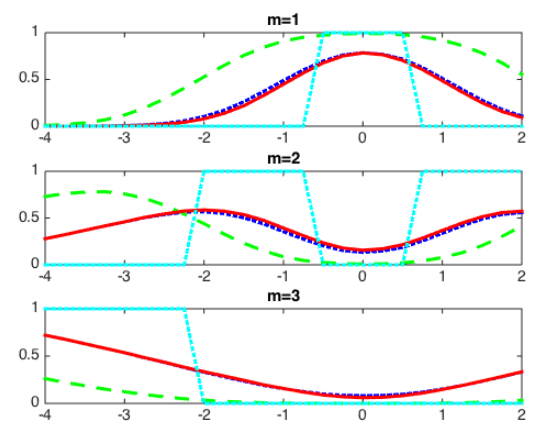

(d) $h=3, T=1000$.
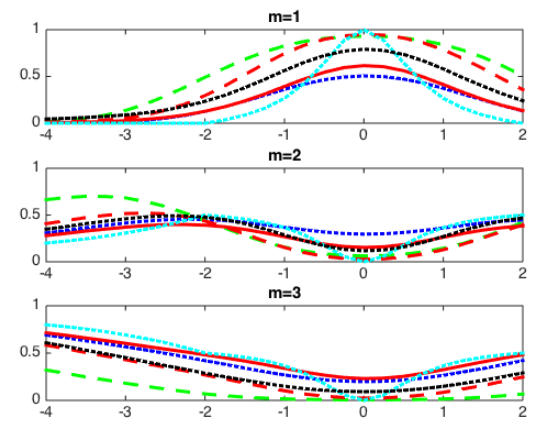

(f) $h=3, T=1000$.

Figure 2.7: (a)-(b) The empirical MSE of the OLS estimator of the model with 1 lag (OLS1), 2 lags (OLS2) and the full model with 3 lags (OLS3). Gray lines show the asymptotic MSE approximations as provided by the delta method. (c)-(d) The empirical selection probabilities (see Figure 2.5 for the appropriate legend). (e)-(f) The empirical distribution of the weights (see Figure 2.6 for the appropriate legend). The DGP is $y_{T, t}=0.5 y_{T, t-1}+\frac{\delta}{\sqrt{T}} y_{T, t-2}+\frac{\delta}{2 \sqrt{T}} y_{T, t-3}+u_{t}$ for all graphs. 

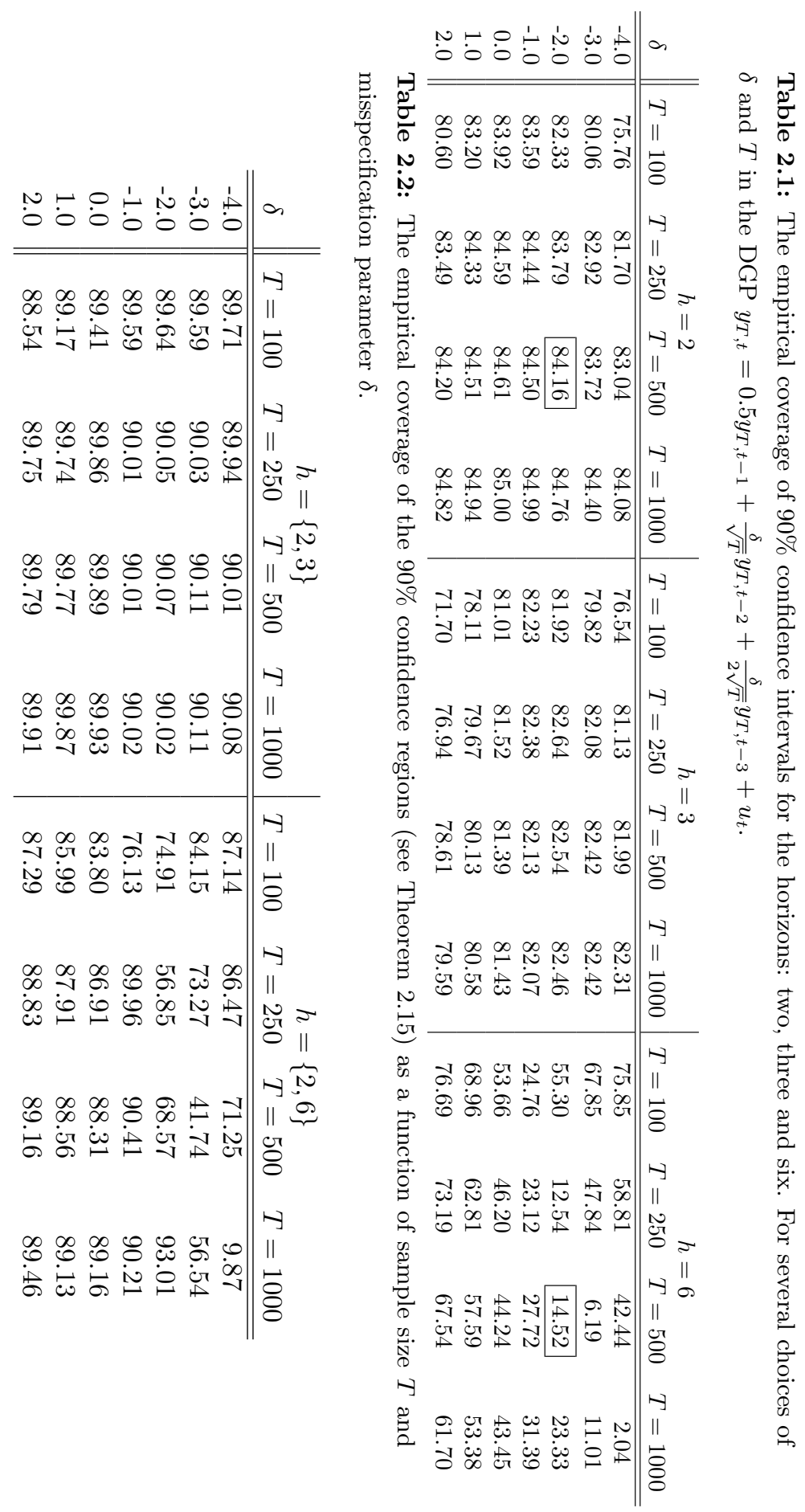


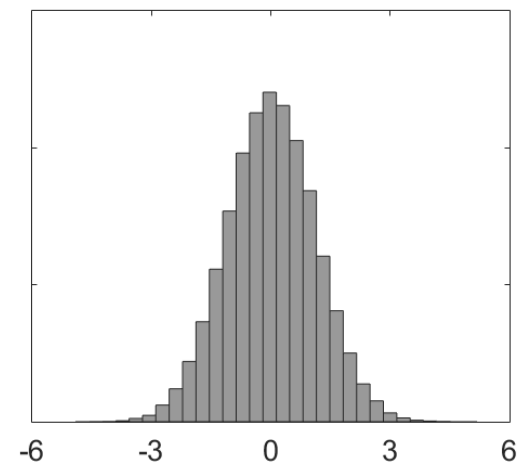

(a) $h=2, T=500$.

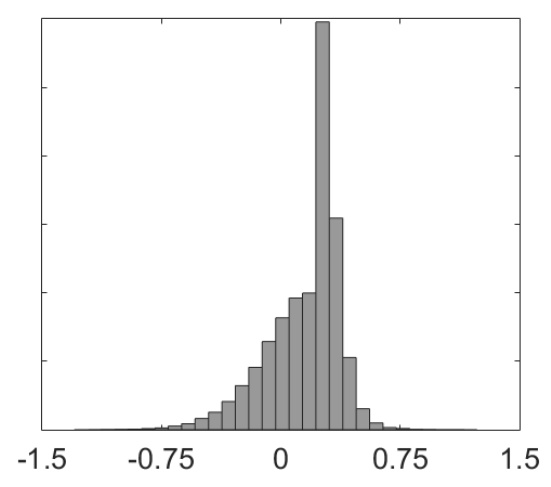

(b) $h=6, T=500$.

Figure 2.8: The confidence intervals are based on the asymptotic normality of $\sqrt{T}\left(\overline{\boldsymbol{\mu}}(\hat{\boldsymbol{w}})-\boldsymbol{\mu}\left(\boldsymbol{\theta}_{T}, \boldsymbol{\sigma}\right)\right)-\boldsymbol{D}_{\theta} \sum_{m=p_{1}}^{p_{1}+p_{2}} \hat{w}_{m} \hat{\boldsymbol{C}}_{m} \hat{\boldsymbol{\delta}}$ (see Theorem 2.15). The displayed histograms are constructed for $y_{T, t}=0.5 y_{T, t-1}+$ $\frac{\delta}{\sqrt{T}} y_{T, t-2}+\frac{\delta}{2 \sqrt{T}} y_{T, t-3}+u_{t}$ with $\delta=-2$ and $T=500$, i.e. the boxed entries in Table 2.1. The number of Monte Carlo replications is 100, 000.

\subsection{Conclusions}

In this chapter we study the issue of model selection and model averaging for multivariate autoregressive processes in a locally drifting asymptotic framework. Within this drifting framework we derive asymptotic normality of the least squares estimators. This asymptotic normality is shown to carry over to sufficiently smooth parameter transformations, e.g. impulse responses, by applying the multivariate delta method. We define the focused information criterion and plug-in averaging estimator as the minimizers of the estimated asymptotic mean squared error of the focus parameter estimator.

We highlight the role of the misspecification parameter $\boldsymbol{\delta}$. Both Liu (2015) and DiTraglia (2016) mentioned that the feasible FIC remains random in the limit. We provide the explicit expressions for the limiting distribution of the FIC values and the elements of the weighting matrices, and illustrate that the feasible estimators do not truly minimize the asymptotic mean squared error. This latter result might encourage further research into different ways to deal with the misspecification parameter. There are to the best of our knowledge two alternatives reported in the literature. The recent paper by Kitagawa and Muris (2016) adopts a mixed frequentist and Bayesian framework to alleviate the estimation of $\delta$ in their study of model averaging in semiparametric estimation of treatment effects. Hansen (2016) similarly adopts a local-to-zero framework but minimizes a risk quantity that does not require the direct estimation of $\delta$.

Our simulation study of univariate and multivariate autoregressive processes clearly shows the role of $\boldsymbol{\delta}$, because the infeasible estimator (the estimator that 


\section{CHAPTER 2}

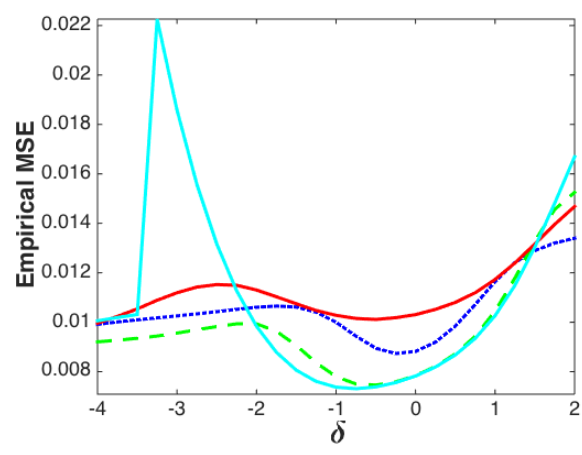

(a) $h=2$, model selection.

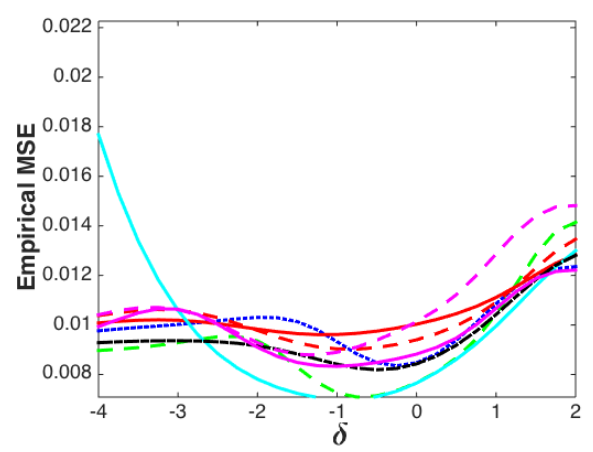

(c) $h=2$, model averaging.

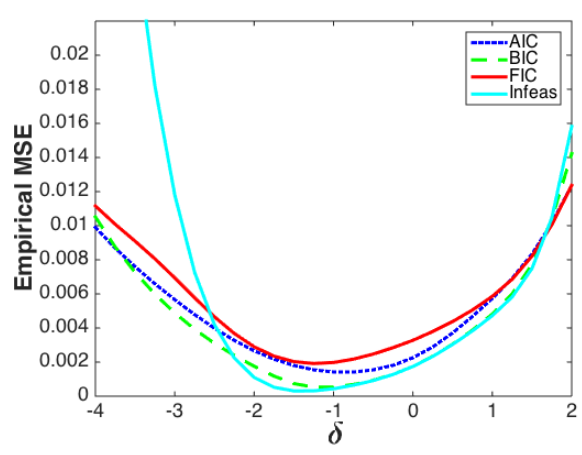

(b) $h=6$, model selection.

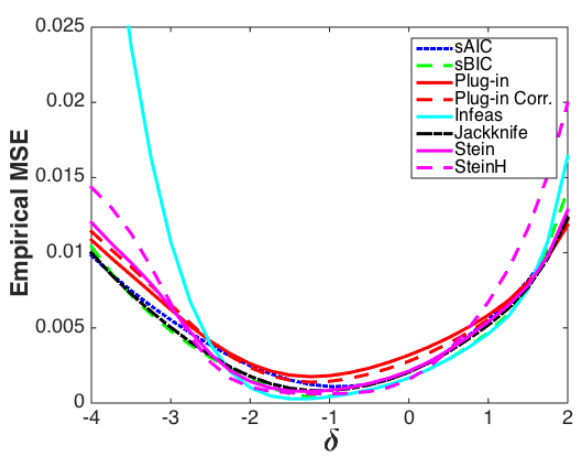

(d) $h=6$, model averaging.

Figure 2.9: The empirical MSE of the impulse response estimator for several selection and averaging methods. We have displayed the results for the response of variable 1 to a structural shock in the variable 1 for horizons 2 and 6. The DGP is given in Equation (2.15). The sample size is $T=100$.

uses the true $\boldsymbol{\delta}$ as if it was known to the researcher) frequently dominates the other methods. The latter is especially the case at the larger sample size of $T=1000$. There is no clearly preferred method for feasible model selection/averaging.

A possible extension of this work is an application to forecasting. Such an extension would complement (1) the predictive static regression setup discussed in Liu and Kuo (2016), and (2) the prediction focused model selection of autoregressive models in Claeskens et al. (2007). Forecasts for autoregressive models often start from the assumption that estimation and prediction are applied to two independent processes with the same stochastic structure. The link to this current chapter is that, under this independence assumption, the asymptotic covariance matrix of the forecast is a continuous transformation of the autoregressive parameters, see section 3.5 of Lütkepohl (2005) for further details. 


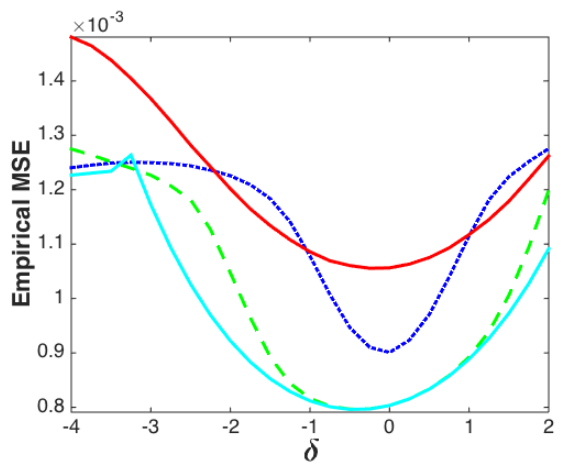

(a) $h=2$, model selection.

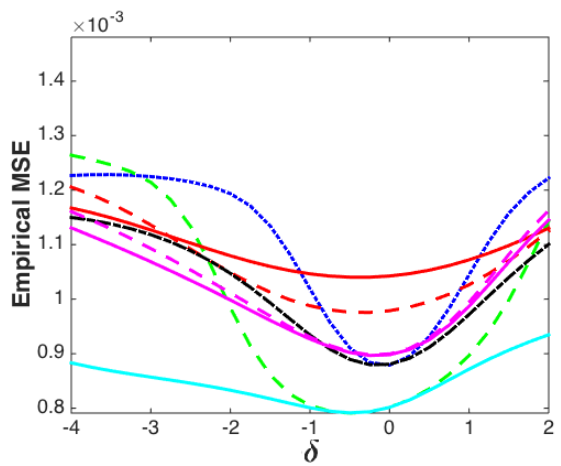

(c) $h=2$, model averaging.

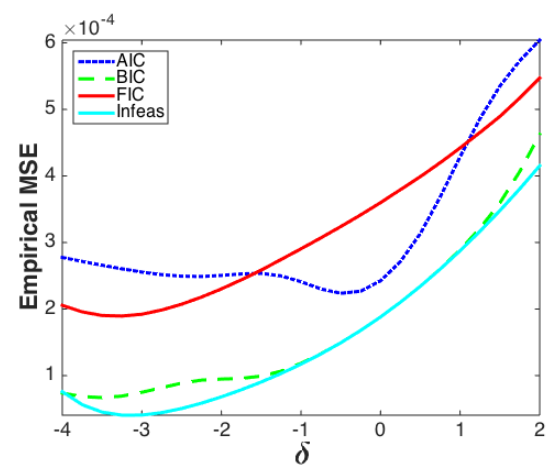

(b) $h=6$, model selection.

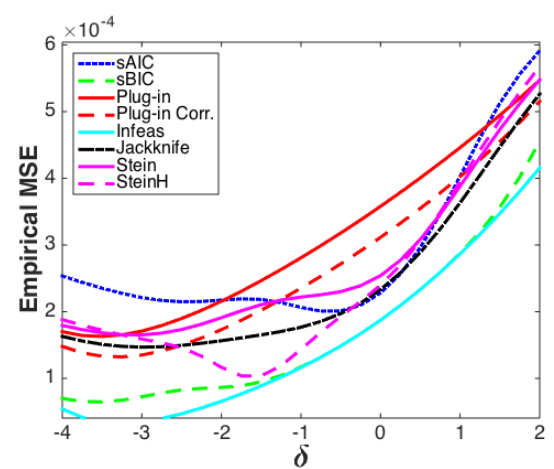

(d) $h=6$, model averaging.

Figure 2.10: Identical to Figure 2.9, but for $T=1000$.

\section{A Appendix}

As in Liu (2015) we first relate the parameters of the $\operatorname{VAR}(m)$ models to the parameters of the $\operatorname{VAR}(p)$. With the aid of the selection matrices we have

$$
\begin{aligned}
& \hat{\boldsymbol{\Theta}}_{T, m}=\boldsymbol{Y}_{T} \boldsymbol{Z}_{T, m}^{\prime}\left(\boldsymbol{Z}_{T, m} \boldsymbol{Z}_{T, m}^{\prime}\right)^{-1}=\left(\boldsymbol{B} \boldsymbol{L}^{\prime} \boldsymbol{Z}_{T}+\boldsymbol{C}_{T} \boldsymbol{S}_{0}^{\prime} \boldsymbol{Z}_{T}+\boldsymbol{U}\right) \boldsymbol{Z}_{T, m}^{\prime}\left(\boldsymbol{Z}_{T, m} \boldsymbol{Z}_{T, m}^{\prime}\right)^{-1} \\
& =\left(\left(\boldsymbol{B}, \boldsymbol{C}_{T}\right) \boldsymbol{S}_{m} \boldsymbol{S}_{m}^{\prime} \boldsymbol{Z}_{T}+\boldsymbol{C}_{T}\left(\boldsymbol{I}_{K p_{2}}-\boldsymbol{\Pi}_{m}^{\prime} \boldsymbol{\Pi}_{m}\right) \boldsymbol{S}_{0}^{\prime} \boldsymbol{Z}_{T}+\boldsymbol{U}\right) \boldsymbol{Z}_{T, m}^{\prime}\left(\boldsymbol{Z}_{T, m} \boldsymbol{Z}_{T, m}^{\prime}\right)^{-1} \\
& =\left(\boldsymbol{\Theta}_{T, m} \boldsymbol{Z}_{T, m}+\boldsymbol{C}_{T}\left(\boldsymbol{I}_{K p_{2}}-\boldsymbol{\Pi}_{m}^{\prime} \boldsymbol{\Pi}_{m}\right) \boldsymbol{S}_{0}^{\prime} \boldsymbol{Z}_{T}+\boldsymbol{U}\right) \boldsymbol{Z}_{T, m}^{\prime}\left(\boldsymbol{Z}_{T, m} \boldsymbol{Z}_{T, m}^{\prime}\right)^{-1} \\
& =\boldsymbol{\Theta}_{T, m}+\boldsymbol{C}_{T}\left(\boldsymbol{I}_{K p_{2}}-\boldsymbol{\Pi}_{m}^{\prime} \boldsymbol{\Pi}_{m}\right) \boldsymbol{S}_{0}^{\prime} \boldsymbol{Z}_{T} \boldsymbol{Z}_{T, m}^{\prime}\left(\boldsymbol{Z}_{T, m} \boldsymbol{Z}_{T, m}^{\prime}\right)^{-1}+\boldsymbol{U} \boldsymbol{Z}_{T, m}^{\prime}\left(\boldsymbol{Z}_{T, m} \boldsymbol{Z}_{T, m}^{\prime}\right)^{-1}
\end{aligned}
$$

Dimensions of a Matrix For convenience we list the dimensions of a matrix used in the proofs.

$$
\boldsymbol{S}_{0}^{\prime} \boldsymbol{\Omega}^{-1}: K p_{2} \times K p
$$




\section{A.1 No Gradient Dependence in Simplified Model}

For $y_{t}=\beta y_{t-1}+\frac{\delta}{\sqrt{T}} y_{t-2}+u_{t}$ we have $\boldsymbol{\Omega}=\frac{\sigma^{2}}{1-\beta^{2}}\left(\begin{array}{cc}1 & \beta \\ \beta & 1\end{array}\right)$ and $\boldsymbol{\Omega}^{-1}=\frac{1}{\sigma^{2}}\left(\begin{array}{cc}1 & -\beta \\ -\beta & 1\end{array}\right)$. The required selection matrices are $\boldsymbol{S}_{1}=\left(\begin{array}{c}1 \\ 0\end{array}\right), \boldsymbol{S}_{0}=\left(\begin{array}{l}0 \\ 1\end{array}\right)$, and $\boldsymbol{\Pi}_{1}^{\prime} \boldsymbol{\Pi}_{1}=\mathbf{O}_{2 \times 2}$. Then

$$
\begin{aligned}
\boldsymbol{C}_{1} & =\left(\boldsymbol{S}_{1}\left[\boldsymbol{S}_{1}^{\prime} \boldsymbol{\Omega} \boldsymbol{S}_{1}\right]^{-1} \boldsymbol{S}_{1}^{\prime} \boldsymbol{\Omega}-\boldsymbol{I}_{K p}\right) \boldsymbol{S}_{0}\left(\boldsymbol{I}_{K p_{2}}-\boldsymbol{\Pi}_{1}^{\prime} \boldsymbol{\Pi}_{1}\right) \otimes \boldsymbol{I}_{K} \\
& =\left(\left(\begin{array}{cc}
1 & \beta \\
0 & 0
\end{array}\right)-\boldsymbol{I}_{2}\right)\left(\begin{array}{c}
0 \\
1
\end{array}\right)=\left(\begin{array}{c}
\beta \\
-1
\end{array}\right):=\boldsymbol{x},
\end{aligned}
$$

and

$$
\begin{aligned}
& \boldsymbol{\Omega}^{-1}-\boldsymbol{P}_{1}=\boldsymbol{\Omega}^{-1}-\boldsymbol{S}_{1}\left(\boldsymbol{S}_{1}^{\prime} \boldsymbol{\Omega} \boldsymbol{S}_{1}\right)^{-1} \boldsymbol{S}_{1}^{\prime} \\
& =\frac{1}{\sigma^{2}}\left(\begin{array}{cc}
1 & -\beta \\
-\beta & 1
\end{array}\right)-\frac{1-\beta^{2}}{\sigma^{2}}\left(\begin{array}{ll}
1 & 0 \\
0 & 0
\end{array}\right) \\
& =\frac{1}{\sigma^{2}}\left(\begin{array}{cc}
\beta^{2} & -\beta \\
-\beta & 1
\end{array}\right)=\frac{1}{\sigma^{2}}\left(\begin{array}{c}
\beta \\
-1
\end{array}\right)(\beta,-1)=\frac{1}{\sigma^{2}} \boldsymbol{x} \boldsymbol{x}^{\prime} \text {. }
\end{aligned}
$$

The elements of the weighting matrix are now given by:

$$
\begin{aligned}
\boldsymbol{\Psi}_{11}^{\text {Biased }} & =\boldsymbol{D}_{\theta}\left[\boldsymbol{C}_{1} \boldsymbol{\delta} \boldsymbol{\delta}^{\prime} \boldsymbol{C}_{1}^{\prime}+\sigma^{2} \boldsymbol{P}_{1}\right] \boldsymbol{D}_{\theta}^{\prime}=\delta^{2}\left(\boldsymbol{D}_{\theta} \boldsymbol{x}\right)^{2}+\sigma^{2} \boldsymbol{D}_{\theta} \boldsymbol{P}_{1} \boldsymbol{D}_{\theta}^{\prime}, \\
\boldsymbol{\Psi}_{11}^{\text {Bias cor }} & =\boldsymbol{D}_{\theta}\left[\boldsymbol{C}_{1}\left(\boldsymbol{\delta} \boldsymbol{\delta}-\sigma^{2} \boldsymbol{S}_{0}^{\prime} \boldsymbol{\Omega}^{-1} \boldsymbol{S}_{0}\right)^{\prime} \boldsymbol{C}_{1}^{\prime}+\sigma^{2} \boldsymbol{P}_{1}\right] \boldsymbol{D}_{\theta}^{\prime} \\
& =\left(\delta^{2}-1\right)\left(\boldsymbol{D}_{\theta} \boldsymbol{x}\right)^{2}+\sigma^{2} \boldsymbol{D}_{\theta} \boldsymbol{P}_{1} \boldsymbol{D}_{\theta}^{\prime}, \\
\boldsymbol{\Psi}_{12} & =\sigma^{2} \boldsymbol{D}_{\theta} \boldsymbol{P}_{1} \boldsymbol{D}_{\theta}^{\prime}, \\
\boldsymbol{\Psi}_{22} & =\sigma^{2} \boldsymbol{D}_{\theta}\left(\boldsymbol{\Omega}^{-1}-\boldsymbol{P}_{1}\right) \boldsymbol{D}_{\theta}^{\prime}+\sigma^{2} \boldsymbol{D}_{\theta} \boldsymbol{P}_{1} \boldsymbol{D}_{\theta}^{\prime}=\left(\boldsymbol{D}_{\theta} \boldsymbol{x}\right)^{2}+\sigma^{2} \boldsymbol{D}_{\theta} \boldsymbol{P}_{1} \boldsymbol{D}_{\theta}^{\prime} .
\end{aligned}
$$

The weights are determined from

$$
\begin{aligned}
\boldsymbol{w}^{0} & =\underset{\boldsymbol{w} \in \mathcal{H}}{\arg \min } \boldsymbol{w}^{\prime} \boldsymbol{\Psi} \boldsymbol{w}=\underset{\boldsymbol{w} \in \mathcal{H}}{\arg \min }\left(\boldsymbol{D}_{\theta} \boldsymbol{x}\right)^{2} \boldsymbol{w}^{\prime}\left[\begin{array}{cc}
\left(\delta^{2}-1\right) & 0 \\
0 & 1
\end{array}\right] \boldsymbol{w}+\sigma^{2} \boldsymbol{D}_{\theta} \boldsymbol{P}_{1} \boldsymbol{D}_{\theta}^{\prime} \\
& =\underset{0 \leq w \leq 1}{\arg \min }\left(\boldsymbol{D}_{\theta} \boldsymbol{x}\right)^{2}\left[w^{2}\left(\delta^{2}-1\right)+(1-w)^{2}\right]+\sigma^{2} \boldsymbol{D}_{\theta} \boldsymbol{P}_{1} \boldsymbol{D}_{\theta}^{\prime}
\end{aligned}
$$

in the case of bias correction, and with $\delta^{2}-1$ replaced by $\delta^{2}$ in the case without. This expression shows that the weights do not depend on the quantity of interest because $\boldsymbol{D}_{\theta}$ is no longer of importance for the optimal weight calculation.

\section{A.2 Proof of Theorem 2.4}

For proving Part (a) we prove a law of large numbers and a central limit theorem in the following.

Law of Large Numbers. We start with the proof of plim $\frac{1}{T} \boldsymbol{Z}_{T} \boldsymbol{Z}_{T}^{\prime}=\boldsymbol{\Omega}$. The process $\left\{\boldsymbol{y}_{T, t}\right\}_{t=-\infty}^{\infty}$ is stationary and ergodic for every fixed $T$ in view of Assumptions 2.1 and 2.2 (cf. e.g. Theorem 3 on page 204 of Hannan (1970)). Define the 
companion matrix $\boldsymbol{A}_{T}$ and innovation vector $\boldsymbol{E}_{t}$ such that $\boldsymbol{z}_{T, t}=\boldsymbol{A}_{T} \boldsymbol{z}_{T, t-1}+\boldsymbol{E}_{t}$, i.e.

$$
\begin{aligned}
\boldsymbol{A}_{T}: & :=\left[\begin{array}{cccccccccc}
\boldsymbol{B}_{1} & \boldsymbol{B}_{2} & \cdots & \boldsymbol{B}_{p_{1}-1} & \boldsymbol{B}_{p_{1}} & \frac{\boldsymbol{C}_{1}}{\sqrt{T}} & \frac{\boldsymbol{C}_{2}}{\sqrt{T}} & \cdots & \frac{\boldsymbol{C}_{p_{2}-1}}{\sqrt{T}} & \frac{\boldsymbol{C}_{p_{2}}}{\sqrt{T}} \\
\boldsymbol{I}_{K} & \mathbf{O} & \cdots & \mathbf{O} & \mathbf{O} & \mathbf{O} & \mathbf{O} & \cdots & \mathbf{O} & \mathbf{O} \\
\vdots & \vdots & & \vdots & \vdots & \vdots & \vdots & & \vdots & \vdots \\
\mathbf{O} & \mathbf{O} & \cdots & \mathbf{O} & \mathbf{O} & \mathbf{O} & \mathbf{O} & \cdots & \boldsymbol{I}_{K} & \mathbf{O}
\end{array}\right]:(K p \times K p), \\
\boldsymbol{E}_{t}: & =\left(\boldsymbol{u}_{t}^{\prime}, \mathbf{0}^{\prime}, \ldots, \mathbf{0}^{\prime}\right)^{\prime}:(K p \times 1) .
\end{aligned}
$$

From this extended VAR(1) form we conclude that

$$
\begin{aligned}
& \boldsymbol{z}_{T, t} \boldsymbol{z}_{T, t}^{\prime}=\boldsymbol{A}_{T} \boldsymbol{z}_{T, t-1} \boldsymbol{z}_{T, t-1}^{\prime} \boldsymbol{A}_{T}^{\prime}+\boldsymbol{A}_{T} \boldsymbol{z}_{T, t-1} \boldsymbol{E}_{t}^{\prime}+\boldsymbol{E}_{t} \boldsymbol{z}_{T, t-1}^{\prime} \boldsymbol{A}_{T}^{\prime}+\boldsymbol{E}_{t} \boldsymbol{E}_{t}^{\prime}, \\
& \boldsymbol{z}_{T, t} \boldsymbol{z}_{T, t}^{\prime}-\boldsymbol{A}_{T} \boldsymbol{z}_{T, t-1} \boldsymbol{z}_{T, t-1}^{\prime} \boldsymbol{A}_{T}^{\prime}=\boldsymbol{A}_{T} \boldsymbol{z}_{T, t-1} \boldsymbol{E}_{t}^{\prime}+\boldsymbol{E}_{t} \boldsymbol{z}_{T, t-1}^{\prime} \boldsymbol{A}_{T}^{\prime}+\boldsymbol{E}_{t} \boldsymbol{E}_{t}^{\prime},
\end{aligned}
$$

where the second line is merely a rearrangement of the first. From Rule (2) of p.662 of Lütkepohl (2005) for the vec operator follows

$$
\operatorname{vec}\left(\boldsymbol{A}_{T} \boldsymbol{z}_{T, t-1} \boldsymbol{z}_{T, t-1}^{\prime} \boldsymbol{A}_{T}^{\prime}\right)=\left(\boldsymbol{A}_{T} \otimes \boldsymbol{A}_{T}\right) \operatorname{vec}\left(\boldsymbol{z}_{T, t-1} \boldsymbol{z}_{T, t-1}^{\prime}\right) .
$$

Now summing over $t$ and taking the limit, because of stationarity we find

$$
\begin{aligned}
& \lim _{T \rightarrow \infty}\left(\boldsymbol{I}_{K^{2} p^{2}}-\boldsymbol{A}_{T} \otimes \boldsymbol{A}_{T}\right) \underset{T \rightarrow \infty}{\operatorname{plim} \operatorname{vec}}\left(\frac{1}{T} \sum_{t=1}^{T} \boldsymbol{z}_{T, t-1} \boldsymbol{z}_{T, t-1}^{\prime}\right) \\
& =\operatorname{plim}_{T \rightarrow \infty} \operatorname{vec}\left(\frac{1}{T} \sum_{t=1}^{T} \boldsymbol{A}_{T} \boldsymbol{z}_{T, t-1} \boldsymbol{E}_{t}^{\prime}\right)+\operatorname{plim}_{T \rightarrow \infty} \operatorname{vec}\left(\frac{1}{T} \sum_{t=1}^{T} \boldsymbol{E}_{t} \boldsymbol{z}_{T, t-1}^{\prime} \boldsymbol{A}_{T}^{\prime}\right) \\
& \quad+\operatorname{plim}_{T \rightarrow \infty} \operatorname{vec}\left(\frac{1}{T} \sum_{t=1}^{T} \boldsymbol{E}_{t} \boldsymbol{E}_{t}^{\prime}\right) .
\end{aligned}
$$

We denote the limit of the nonrandom matrix $\boldsymbol{A}_{T}$ for large $T$ by $\boldsymbol{A}_{\infty} . \boldsymbol{A}_{\infty}$ is thus obtained from $\boldsymbol{A}_{T}$ by replacing the ratios $\boldsymbol{C}_{i} / \sqrt{T}$ with zero matrices for $i \in\left\{1,2, \ldots, p_{2}\right\}$. Note that the eigenvalues of $\boldsymbol{A}_{\infty}$ coincide with the roots of the matrix polynomial $\boldsymbol{B}_{\infty}(z)$ augmented with $K p_{2}$ additional zero eigenvalues. Hence, Assumption 2.3 guarantees that the matrix $\boldsymbol{I}_{K^{2} p^{2}}-\boldsymbol{A}_{\infty} \otimes \boldsymbol{A}_{\infty}$ is invertible.

We now consider the RHS of Equation (2.18). Let $y_{T, t-j, k}$ and $u_{t, k}$ denote the $k^{\prime}$ th component of $\boldsymbol{y}_{T, t-j}$ and $\boldsymbol{u}_{t}$, respectively. If we can show that $\frac{1}{T} \sum_{t=1}^{T} y_{T, t-j, k} u_{t, l} \stackrel{p}{\longrightarrow} 0$ for all $j \in\{1,2, \ldots, p\}$ and $k, l \in\{1,2, \ldots, K\}$, then the first two terms on the RHS of Equation (2.18) are $o_{p}(1)$. To prove this we define the array $X_{T, t}^{j k l}=y_{T, t-j, k} u_{t, l} / T$ and the norming $c_{T}=1 / T . X_{T, t}^{j k l}$ is a martingale difference (m.d.) array with respect to the filtration $\mathcal{F}_{t}=\sigma\left(\boldsymbol{u}_{s},-\infty<s \leq t\right)$ and $\mathrm{E}\left|X_{T, t}^{j k l} / c_{T}\right|^{4}=\mathrm{E}\left|y_{T, t-j, k} u_{t, l}\right|^{4}$ is finite in view of Assumption 2.1. Theorem 12.10 from Davidson (1994) implies that $\left|X_{T, t}^{j k l}\right|^{2}$ is uniformly integrable and Theorem 19.7 from the same reference then gives $\frac{1}{T} \sum_{t=1}^{T} y_{T, t-j, k} u_{t} \stackrel{L_{2}}{\rightarrow} 0$. The result for the first two terms follows. The third term on the RHS of Equation (2.18) is a sample mean of an i.i.d. sequence. Khinchine's Theorem (Theorem 23.5 of Davidson (1994)) gives the probability limit. Combining all the results, we conclude that 


\section{CHAPTER 2}

$$
\begin{aligned}
\operatorname{vec}(\boldsymbol{\Omega}):= & \operatorname{plim}_{T \rightarrow \infty} \operatorname{vec}\left(\frac{1}{T} \sum_{t=1}^{T} \boldsymbol{z}_{T, t-1} \boldsymbol{z}_{T, t-1}^{\prime}\right)=\left(\boldsymbol{I}_{K^{2} p^{2}}-\boldsymbol{A}_{\infty} \otimes \boldsymbol{A}_{\infty}\right)^{-1} \operatorname{vec}\left(\boldsymbol{\Sigma}^{*}\right) \\
& +o_{p}(1)
\end{aligned}
$$

where $\boldsymbol{\Sigma}^{*}=\boldsymbol{e} \boldsymbol{\Sigma} \boldsymbol{e}^{\prime}$ and $\boldsymbol{e}$ is the $(K p \times K)$ matrix composed of the first $K$ columns of $I_{K p}$. This shows that $\operatorname{plim}_{T \rightarrow \infty} \frac{1}{T} \boldsymbol{Z}_{T} \boldsymbol{Z}_{T}^{\prime}=\boldsymbol{\Omega}$ exists. Ergodicity for every $T$ also provides the result $\left(\boldsymbol{I}_{K^{2} p^{2}}-\boldsymbol{A}_{T} \otimes \boldsymbol{A}_{T}\right) \operatorname{vec}\left(\mathrm{E}\left(\boldsymbol{z}_{T, t-1} \boldsymbol{z}_{T, t-1}^{\prime}\right)\right)=\operatorname{vec}\left(\boldsymbol{\Sigma}^{*}\right)$ such that analogously $\boldsymbol{\Omega}=\lim _{T \rightarrow \infty} \mathrm{E}\left(\boldsymbol{z}_{T, t-1} \boldsymbol{z}_{T, t-1}^{\prime}\right)$ follows, because $\boldsymbol{A}_{T} \rightarrow \boldsymbol{A}_{\infty}$.

Central Limit Theorem. We rely on the Cramér-Wold theorem (e.g. Result 25.5 from Davidson (1994)) to prove the convergence of $\frac{1}{\sqrt{T}} \sum_{t=1}^{T} \operatorname{vec}\left(\boldsymbol{u}_{t} \boldsymbol{z}_{T, t-1}^{\prime}\right)$ to $\boldsymbol{R} \sim \mathrm{N}(\mathbf{0}, \boldsymbol{\Omega} \otimes \boldsymbol{\Sigma})$. Let $\boldsymbol{\xi}$ denote a fixed $\left(K^{2} p \times 1\right)$ vector. $X_{T, t}^{*}=\boldsymbol{\xi}^{\prime} \operatorname{vec}\left(\boldsymbol{u}_{t} \boldsymbol{z}_{T, t-1}^{\prime}\right)$ is a m.d. array with respect to $\mathcal{F}_{t}$. We note that

$$
\begin{aligned}
\sigma_{T, t}^{2}: & =\mathrm{E}\left(X_{T, t}^{* 2} \mid \mathcal{F}_{t-1}\right)=\mathrm{E}\left(\boldsymbol{\xi}^{\prime} \operatorname{vec}\left(\boldsymbol{u}_{t} \boldsymbol{z}_{T, t-1}^{\prime}\right) \operatorname{vec}\left(\boldsymbol{u}_{t} \boldsymbol{z}_{T, t-1}^{\prime}\right)^{\prime} \boldsymbol{\xi} \mid \mathcal{F}_{t-1}\right) \\
& =\mathrm{E}\left(\boldsymbol{\xi}^{\prime}\left(\left(\boldsymbol{z}_{T, t-1} \boldsymbol{z}_{T, t-1}^{\prime}\right) \otimes\left(\boldsymbol{u}_{t} \boldsymbol{u}_{t}^{\prime}\right)\right) \boldsymbol{\xi} \mid \mathcal{F}_{t-1}\right)=\boldsymbol{\xi}^{\prime}\left(\left(\boldsymbol{z}_{T, t-1} \boldsymbol{z}_{T, t-1}^{\prime}\right) \otimes \boldsymbol{\Sigma}\right) \boldsymbol{\xi}
\end{aligned}
$$

and that $X_{T, t}^{* 2}$ is square integrable by Assumption 2.1. Moreover, from $s_{T}^{2}:=$ $\sum_{t=1}^{T} \mathrm{E}\left(X_{T, t}^{* 2}\right)=T \boldsymbol{\xi}^{\prime}\left(\mathrm{E}\left(\boldsymbol{z}_{T, t-1} \boldsymbol{z}_{T, t-1}^{\prime}\right) \otimes \boldsymbol{\Sigma}\right) \boldsymbol{\xi}$ we get

$$
\sup _{T} \frac{T}{s_{T}^{2}}=\sup _{T} \frac{1}{\boldsymbol{\xi}^{\prime}\left(\mathrm{E}\left(\boldsymbol{z}_{T, t-1} \boldsymbol{z}_{T, t-1}^{\prime}\right) \otimes \boldsymbol{\Sigma}\right) \boldsymbol{\xi}}<\infty
$$

because the quadratic form in the denominator cannot be zero as both $\boldsymbol{\Sigma}$ and $\mathrm{E}\left(\boldsymbol{z}_{T, t-1} \boldsymbol{z}_{T, t-1}^{\prime}\right)$ are positive definite matrices: The positive definiteness of $\boldsymbol{\Sigma}$ is part of Assumption 2.1. For finite $T, \mathrm{E}\left(\boldsymbol{z}_{T, t-1} \boldsymbol{z}_{T, t-1}^{\prime}\right)$ there cannot exist a $\boldsymbol{\kappa} \neq \mathbf{0}$ such that $\boldsymbol{\kappa}^{\prime} \mathrm{E}\left(\boldsymbol{z}_{T, t-1} \boldsymbol{z}_{T, t-1}^{\prime}\right) \boldsymbol{\kappa}=0$ as this is equal to $\mathrm{E}\left(\boldsymbol{\kappa}^{\prime} \boldsymbol{z}_{T, t-1}\right)^{2}$ and this would imply at least one component of $\boldsymbol{z}_{T, t-1}$ being zero for all $t$. But this would be a contradiction to the process being random, i.e. to $\boldsymbol{\Sigma}$ being positive definite. Also, for the case where $T$ is not finite such a $\boldsymbol{\kappa}$ cannot exist as $\mathrm{E}\left(\boldsymbol{z}_{T, t-1} \boldsymbol{z}_{T, t-1}^{\prime}\right)$ tends to the positive definite $\boldsymbol{\Omega}$. Hence, we have shown positive definiteness. Now, a generalization of Theorem 24.4 from Davidson (1994) to martingale difference arrays shows that

$$
\frac{\frac{1}{\sqrt{T}} \sum_{t=1}^{T} \boldsymbol{\xi}^{\prime} \operatorname{vec}\left(\boldsymbol{u}_{t} \boldsymbol{z}_{T, t-1}^{\prime}\right)}{\sqrt{\boldsymbol{\xi}^{\prime}\left(\mathrm{E}\left(\boldsymbol{z}_{T, t-1} \boldsymbol{z}_{T, t-1}^{\prime}\right) \otimes \boldsymbol{\Sigma}\right) \boldsymbol{\xi}}} \stackrel{d}{\longrightarrow} \mathrm{N}(0,1) .
$$

The expression under the square root is asymptotically equivalent to $\boldsymbol{\xi}^{\prime}(\boldsymbol{\Omega} \otimes \boldsymbol{\Sigma}) \boldsymbol{\xi}$. The second result, $\frac{1}{\sqrt{T}} \sum_{t=1}^{T} \operatorname{vec}\left(\boldsymbol{u}_{t} \boldsymbol{z}_{T, t-1}^{\prime}\right) \stackrel{d}{\longrightarrow} \mathrm{N}(\mathbf{0}, \boldsymbol{\Omega} \otimes \boldsymbol{\Sigma})$, follows according to Crámer and Wold because $\boldsymbol{\xi}$ is arbitrary. The proof of part (a) is complete. 
Part (b). This is a joint convergence result of the parameter estimators with the estimator for the covariance matrix. Let $m \in \mathcal{M}=\left\{p_{1}, p_{1}+1, \ldots, p\right\}$. For the estimated residual matrix $\hat{\boldsymbol{U}}_{T, m}$ of model $m$ we have

$$
\begin{aligned}
\hat{\boldsymbol{U}}_{T, m} & =\boldsymbol{Y}_{T}-\hat{\boldsymbol{\Theta}}_{T, m} \boldsymbol{Z}_{T, m}=\boldsymbol{\Theta}_{T} \boldsymbol{Z}_{T}+\boldsymbol{U}-\hat{\boldsymbol{\Theta}}_{T, m} \boldsymbol{S}_{m}^{\prime} \boldsymbol{Z}_{T} \\
& =\left(\boldsymbol{\Theta}_{T, m} \boldsymbol{S}_{m}^{\prime}+\boldsymbol{C}_{T}\left(\boldsymbol{I}_{K p_{2}}-\boldsymbol{\Pi}_{m}^{\prime} \boldsymbol{\Pi}_{m}\right) \boldsymbol{S}_{0}^{\prime}\right) \boldsymbol{Z}_{T}+\boldsymbol{U}-\hat{\boldsymbol{\Theta}}_{T, m} \boldsymbol{S}_{m}^{\prime} \boldsymbol{Z}_{T} .
\end{aligned}
$$

And the estimated covariance matrix based on the residuals from the model with $m$ lags satisfies

$$
\begin{aligned}
\hat{\boldsymbol{\Sigma}}^{m}=\frac{1}{T} & \hat{\boldsymbol{U}}_{T, m} \hat{\boldsymbol{U}}_{T, m}^{\prime} \\
=\frac{1}{T} & {\left[\left(\boldsymbol{\Theta}_{T, m}-\hat{\boldsymbol{\Theta}}_{T, m}\right) \boldsymbol{S}_{m}^{\prime} \boldsymbol{Z}_{T}+\boldsymbol{C}_{T}\left(\boldsymbol{I}_{K p_{2}}-\boldsymbol{\Pi}_{m}^{\prime} \boldsymbol{\Pi}_{m}\right) \boldsymbol{S}_{0}^{\prime} \boldsymbol{Z}_{T}+\boldsymbol{U}\right] } \\
& \quad\left[\left(\boldsymbol{\Theta}_{T, m}-\hat{\boldsymbol{\Theta}}_{T, m}\right) \boldsymbol{S}_{m}^{\prime} \boldsymbol{Z}_{T}+\boldsymbol{C}_{T}\left(\boldsymbol{I}_{K p_{2}}-\boldsymbol{\Pi}_{m}^{\prime} \boldsymbol{\Pi}_{m}\right) \boldsymbol{S}_{0}^{\prime} \boldsymbol{Z}_{T}+\boldsymbol{U}\right]^{\prime} \\
=( & \left.\boldsymbol{\Theta}_{T, m}-\hat{\boldsymbol{\Theta}}_{T, m}\right) \boldsymbol{S}_{m}^{\prime}\left(\frac{\boldsymbol{Z}_{T} \boldsymbol{Z}_{T}^{\prime}}{T}\right) \boldsymbol{S}_{m}\left(\boldsymbol{\Theta}_{T, m}-\hat{\boldsymbol{\Theta}}_{T, m}\right)^{\prime} \\
& +\boldsymbol{C}_{T}\left(\boldsymbol{I}_{K p_{2}}-\boldsymbol{\Pi}_{m}^{\prime} \boldsymbol{\Pi}_{m}\right) \boldsymbol{S}_{0}^{\prime}\left(\frac{\boldsymbol{Z}_{T} \boldsymbol{Z}_{T}^{\prime}}{T}\right) \boldsymbol{S}_{0}\left(\boldsymbol{I}_{K p_{2}}-\boldsymbol{\Pi}_{m}^{\prime} \boldsymbol{\Pi}_{m}\right)^{\prime} \boldsymbol{C}_{T}^{\prime} \\
& +\left(\boldsymbol{\Theta}_{T, m}-\hat{\boldsymbol{\Theta}}_{T, m}\right) \boldsymbol{S}_{m}^{\prime}\left(\frac{\boldsymbol{Z}_{T} \boldsymbol{Z}_{T}^{\prime}}{T}\right) \boldsymbol{S}_{0}\left(\boldsymbol{I}_{K p_{2}}-\boldsymbol{\Pi}_{m}^{\prime} \boldsymbol{\Pi}_{m}\right)^{\prime} \boldsymbol{C}_{T}^{\prime} \\
& +\left(\boldsymbol{\Theta}_{T, m}-\hat{\boldsymbol{\Theta}}_{T, m}\right) \boldsymbol{S}_{m}^{\prime}\left(\frac{\boldsymbol{Z}_{T} \boldsymbol{U}^{\prime}}{T}\right) \\
& +\boldsymbol{C}_{T}\left(\boldsymbol{I}_{K p_{2}}-\boldsymbol{\Pi}_{m}^{\prime} \boldsymbol{\Pi}_{m}\right) \boldsymbol{S}_{0}^{\prime}\left(\frac{\boldsymbol{Z}_{T} \boldsymbol{Z}_{T}^{\prime}}{T}\right) \boldsymbol{S}_{m}\left(\boldsymbol{\Theta}_{T, m}-\hat{\boldsymbol{\Theta}}_{T, m}\right)^{\prime} \\
& +\boldsymbol{C}_{T}\left(\boldsymbol{I}_{K p_{2}}-\boldsymbol{\Pi}_{m}^{\prime} \boldsymbol{\Pi}_{m}\right) \boldsymbol{S}_{0}^{\prime}\left(\frac{\boldsymbol{Z}_{T} \boldsymbol{U}^{\prime}}{T}\right)+\left(\frac{\boldsymbol{U}_{T}^{\prime}}{T}\right) \boldsymbol{S}_{m}\left(\boldsymbol{\Theta}_{T, m}-\hat{\boldsymbol{\Theta}}_{T, m}\right) \\
& +\left(\frac{\boldsymbol{U} \boldsymbol{Z}_{T}}{T}\right) \boldsymbol{S}_{0}\left(\boldsymbol{I}_{K p_{2}}-\boldsymbol{\Pi}_{m}^{\prime} \boldsymbol{\Pi}_{m}\right)^{\prime} \boldsymbol{C}_{T}^{\prime}+\frac{1}{T} \boldsymbol{U}^{\prime} .
\end{aligned}
$$

The stochastic orders of the various terms in Equation (2.22) are known from previous results. We have $\boldsymbol{\Theta}_{T, m}-\hat{\boldsymbol{\Theta}}_{T, m}=O_{p}\left(T^{-1 / 2}\right), \boldsymbol{Z}_{T} \boldsymbol{Z}_{T}^{\prime} / T \stackrel{p}{\longrightarrow} \boldsymbol{\Omega}, \boldsymbol{C}_{T}=$ $O\left(T^{-1 / 2}\right)$ and $\boldsymbol{Z}_{T} \boldsymbol{U}^{\prime} / T=O_{p}\left(T^{-1 / 2}\right)$ by Equation $(2.21)$. We conclude that $\hat{\boldsymbol{\Sigma}}^{m}=$ $\frac{1}{T} \boldsymbol{U} \boldsymbol{U}^{\prime}+o_{P}(1)$. This means that every covariance estimator (every in the sense of for all $m \in \mathcal{M}$ ) has the same asymptotic distribution as the covariance estimator based on the true innovations.

Joint asymptotic normality of the parameter estimates and the covariance estimator can be obtained along the lines of the proof of Proposition 11.2 of Hamilton (1994). That is, we define

$$
\boldsymbol{\lambda}_{t}=\operatorname{vech}\left(\begin{array}{cccc}
u_{1 t}^{2}-\sigma_{11} & u_{1 t} u_{2 t}-\sigma_{12} & \ldots & u_{1 t} u_{K t}-\sigma_{1 K} \\
u_{2 t} u_{1 t}-\sigma_{21} & u_{2 t}^{2}-\sigma_{22} & \ldots & u_{2 t} u_{K t}-\sigma_{2 K} \\
\vdots & \vdots & \ddots & \vdots \\
u_{K t} u_{1 t}-\sigma_{K 1} & u_{K t} u_{2 t}-\sigma_{K 2} & \ldots & u_{K t}^{2}-\sigma_{K K}
\end{array}\right)
$$




\section{CHAPTER 2}

The sequence $\left\{\boldsymbol{\lambda}_{t}\right\}$ is i.i.d. and thus also a martingale difference sequence. One can apply the Cramer-Wold Theorem to the extended martingale difference vector $\left(\operatorname{vec}\left(\boldsymbol{u}_{t} \boldsymbol{z}_{T, t-1}^{\prime}\right)^{\prime}, \boldsymbol{\lambda}_{t}^{\prime}\right)^{\prime}$ to show

$$
\left[\begin{array}{c}
(1 \sqrt{T}) \sum_{t=1}^{T} \operatorname{vec}\left(\boldsymbol{u}_{t} \boldsymbol{z}_{T, t-1}^{\prime}\right) \\
(1 \sqrt{T}) \sum_{t=1}^{T} \boldsymbol{\lambda}_{t}
\end{array}\right] \stackrel{d}{\longrightarrow} \mathrm{N}\left(\left[\begin{array}{l}
\mathbf{0} \\
\mathbf{0}
\end{array}\right],\left[\begin{array}{ll}
\boldsymbol{\Xi}_{11} & \boldsymbol{\Xi}_{12} \\
\boldsymbol{\Xi}_{21} & \boldsymbol{\Xi}_{22}
\end{array}\right]\right)
$$

We already know that $\boldsymbol{\Xi}_{11}=\boldsymbol{\Omega} \otimes \boldsymbol{\Sigma}$. The elements in the covariance matrix $\boldsymbol{\Xi}_{12}$ take the form $\lim _{T \rightarrow \infty} \mathrm{E}\left(u_{k_{1} t} y_{T, t-j, k_{2}}\left(u_{k_{3} t} u_{k_{4} t}-\sigma_{k_{3} k_{4}}\right)\right)$. They are zero because $\lim _{T \rightarrow \infty} \mathrm{E}\left(y_{T, t-j, k_{2}}\right)=0$. Finally, $\boldsymbol{\Xi}_{22}=\mathrm{E}\left(\boldsymbol{\lambda}_{t} \boldsymbol{\lambda}_{t}^{\prime}\right)$. The typical elements are $\mathrm{E}\left(\left(u_{i t} u_{j t}-\sigma_{i j}\right)\left(u_{l t} u_{m t}-\sigma_{l m}\right)\right)$.

Part (c). Define two independent random vectors: $\boldsymbol{R} \sim \mathrm{N}(\mathbf{0}, \boldsymbol{\Omega} \otimes \boldsymbol{\Sigma})$ and $\boldsymbol{S} \sim$ $\mathrm{N}\left(\mathbf{0}, \boldsymbol{\Xi}_{22}\right)$ of length $K^{2} p$ and $K(K+1) / 2$, respectively. Consider three different models indexed by $m_{1}, m_{2}, m_{3} \in \mathcal{M}$. The proof is immediate, since

$$
\begin{aligned}
{\left[\begin{array}{c}
\sqrt{T}\left(\hat{\boldsymbol{\theta}}_{T, m_{1}}-\boldsymbol{\theta}_{T, m_{1}}\right) \\
\sqrt{T}\left(\hat{\boldsymbol{\theta}}_{T, m_{2}}-\boldsymbol{\theta}_{T, m_{2}}\right) \\
\sqrt{T} \operatorname{vech}\left(\hat{\boldsymbol{\Sigma}}_{u}^{m_{3}}-\boldsymbol{\Sigma}_{u}\right)
\end{array}\right] } & \stackrel{d}{\longrightarrow}\left[\begin{array}{c}
\boldsymbol{A}_{m_{1}} \\
\boldsymbol{A}_{m_{2}} \\
\mathbf{O}
\end{array}\right] \boldsymbol{\delta} \\
+ & {\left[\begin{array}{cc}
\left(\left[\boldsymbol{S}_{m_{1}}^{\prime} \boldsymbol{\Omega} \boldsymbol{S}_{m_{1}}\right]^{-1} \boldsymbol{S}_{m_{1}}^{\prime} \otimes \boldsymbol{I}_{K}\right) & \mathbf{O} \\
\left(\left[\boldsymbol{S}_{m_{2}}^{\prime} \boldsymbol{\Omega} \boldsymbol{S}_{m_{2}}\right]^{-1} \boldsymbol{S}_{m_{2}}^{\prime} \otimes \boldsymbol{I}_{K}\right) & \mathbf{O} \\
\mathbf{O} & \boldsymbol{I}_{K(K+1) / 2}
\end{array}\right]\left[\begin{array}{c}
\boldsymbol{R} \\
\boldsymbol{S}
\end{array}\right] }
\end{aligned}
$$

\section{A.3 Other Proofs}

The following Lemma states that we can obtain the smaller models' impulse response coefficients by plugging zeros into the mapping defined for the full model.

Lemma 2.21. Consider the notation of Section 2.2.3 where $\boldsymbol{\mu}$ describes a particular impulse response coefficient of the full model. Then $\boldsymbol{\mu}\left(\left(\boldsymbol{\theta}_{T, m}, \mathbf{0}_{K^{2}(p-m)}\right), \boldsymbol{\sigma}\right)$ is equivalent to that specific impulse response coefficient of the model with $m$ lags.

Proof. We use mathematical induction and consider the impulse responses of the $\operatorname{VAR}(p)$ and $\operatorname{VAR}(p+1)$ models. For $p=0$ we are comparing a white noise model with a $\operatorname{VAR}(1)$ with coefficient matrix $\boldsymbol{B}$. The impulse responses at horizon $h$ for these models are $\mathbf{O}_{K \times K}$ and $\boldsymbol{B}^{h}$, respectively. The base case $p=0$ holds.

We start the inductive step by defining the companion matrix of the $\operatorname{VAR}(p+1)$,

$$
\boldsymbol{A}_{(p+1)}=\left[\begin{array}{ccccc}
\boldsymbol{B}_{1} & \boldsymbol{B}_{2} & \ldots & \boldsymbol{B}_{p} & \boldsymbol{B}_{p+1} \\
\boldsymbol{I}_{K} & \mathbf{O} & \ldots & \mathbf{O} & \mathbf{O} \\
\mathbf{O} & \boldsymbol{I}_{K} & \ldots & \mathbf{O} & \mathbf{O} \\
\vdots & \vdots & \ddots & \vdots & \vdots \\
\mathbf{O} & \mathbf{O} & \ldots & \boldsymbol{I}_{K} & \mathbf{O}
\end{array}\right]
$$


This companion matrix is $(K(p+1) \times K(p+1))$. For an arbitrary matrix of this size, $\boldsymbol{F}$, let $[\boldsymbol{F}]^{i j}$ denote its $(i, j)$ 'th block of dimension $(K \times K)$. In this notation the impulse response of the $\operatorname{VAR}(p+1)$ at horizon $h$ is simply $\left[\left(\boldsymbol{A}_{p+1}\right)^{h}\right]^{11}$. Setting $\boldsymbol{B}_{p+1}=\mathbf{O}$ provides $K$ zero columns, and hence

$$
\begin{aligned}
{\left[\left(\boldsymbol{A}_{(p+1)}\right)^{h}\right]^{11} } & =\sum_{j_{1}=1}^{p+1} \cdots \sum_{j_{h-1}=1}^{p+1}\left[\boldsymbol{A}_{p+1}\right]^{1 j_{1}}\left[\boldsymbol{A}_{p+1}\right]^{j_{1} j_{2}} \cdots\left[\boldsymbol{A}_{p+1}\right]^{j_{h-1} 1} \\
& =\sum_{j_{1}=1}^{p}\left[\boldsymbol{A}_{p+1}\right]^{1 j_{1}}\left(\sum_{j_{2}=1}^{p+1} \cdots \sum_{j_{h-1}=1}^{p+1}\left[\boldsymbol{A}_{p+1}\right]^{j_{1} j_{2}} \cdots\left[\boldsymbol{A}_{p+1}\right]^{j_{h-1} 1}\right) \\
& =\ldots=\sum_{j_{1}=1}^{p} \cdots \sum_{j_{h-1}=1}^{p}\left[\boldsymbol{A}_{p}\right]^{1 j_{1}}\left[\boldsymbol{A}_{p}\right]^{j_{1} j_{2}} \cdots\left[\boldsymbol{A}_{p}\right]^{j_{h-1} 1}=\left[\left(\boldsymbol{A}_{p}\right)^{h}\right]^{11},
\end{aligned}
$$

where $\boldsymbol{A}_{p}$ is the companion matrix related to the $\operatorname{VAR}(p)$. This completes the inductive step and the proof.

Proof of Theorem 2.7. We first rewrite

$$
\begin{aligned}
& \sqrt{T}\left(\boldsymbol{\mu}\left(\hat{\boldsymbol{\theta}}_{T, m}, \mathbf{0}_{K^{2}(p-m)}, \hat{\boldsymbol{\sigma}}\right)-\boldsymbol{\mu}\left(\boldsymbol{\theta}_{T, p}, \boldsymbol{\sigma}\right)\right) \\
= & \sqrt{T}\left(\boldsymbol{\mu}\left(\hat{\boldsymbol{\theta}}_{T, m}, \mathbf{0}_{K^{2}(p-m)}, \hat{\boldsymbol{\sigma}}\right)-\boldsymbol{\mu}\left(\boldsymbol{\theta}_{T, m}, \mathbf{0}_{K^{2}(p-m)}, \boldsymbol{\sigma}\right)\right) \\
- & \sqrt{T}\left(\boldsymbol{\mu}\left(\boldsymbol{\theta}_{T, p}, \boldsymbol{\sigma}\right)-\boldsymbol{\mu}\left(\boldsymbol{\theta}_{T, m}, \mathbf{0}_{K^{2}(p-m)}, \boldsymbol{\sigma}\right)\right) .
\end{aligned}
$$

The first term on the RHS of Equation (2.25) contains a parameter transformation of the estimated parameters. The first order delta method can be applied to this expression because the theorem explicitly assumes non-vanishing derivatives at the necessary points. The second term is nonrandom. It is the difference of two terms which only differ in locally misspecified coefficients, which are set to zero in the second term. We will use a Taylor expansion on this second contribution. The result from the delta method together with Theorem 2.4 is

$$
\begin{aligned}
& \sqrt{T}\left(\boldsymbol{\mu}\left(\hat{\boldsymbol{\theta}}_{T, m}, \mathbf{0}_{K^{2}(p-m)}, \hat{\boldsymbol{\sigma}}\right)-\boldsymbol{\mu}\left(\boldsymbol{\theta}_{T, m}, \mathbf{0}_{K^{2}(p-m)}, \boldsymbol{\sigma}\right)\right) \\
& \stackrel{d}{\longrightarrow}\left(\frac{\partial \boldsymbol{\mu}\left(\boldsymbol{\theta}_{\infty}, \boldsymbol{\sigma}\right)}{\partial \boldsymbol{\theta}^{\prime}}\left(\boldsymbol{S}_{m} \otimes \boldsymbol{I}_{K}\right)\right)\left(\boldsymbol{A}_{m} \boldsymbol{\delta}+\left(\left[\boldsymbol{S}_{m}^{\prime} \boldsymbol{\Omega} \boldsymbol{S}_{m}\right]^{-1} \boldsymbol{S}_{m}^{\prime} \otimes \boldsymbol{I}_{K}\right) \boldsymbol{R}\right) \\
& +\left(\frac{\partial \boldsymbol{\mu}\left(\boldsymbol{\theta}_{\infty}, \boldsymbol{\sigma}\right)}{\partial \boldsymbol{\sigma}^{\prime}}\right) \boldsymbol{S}
\end{aligned}
$$

where $\boldsymbol{R}$ and $\boldsymbol{S}$ are defined in the proof of Part (c) of Theorem 2.4. The result of the Taylor expansion is

$$
\begin{aligned}
& \sqrt{T}\left(\boldsymbol{\mu}\left(\boldsymbol{\theta}_{T, p}, \boldsymbol{\sigma}\right)-\boldsymbol{\mu}\left(\boldsymbol{\theta}_{T, m}, \mathbf{0}_{K^{2}(p-m)}, \boldsymbol{\sigma}\right)\right) \\
& =\sqrt{T} \boldsymbol{\mu}\left(\boldsymbol{\theta}_{T, m}, \mathbf{0}_{K^{2}(p-m)}, \boldsymbol{\sigma}\right)+\left(\frac{\partial \boldsymbol{\mu}\left(\boldsymbol{\theta}_{\infty}, \boldsymbol{\sigma}\right)}{\partial \boldsymbol{\theta}^{\prime}}\left(\boldsymbol{S}_{0} \otimes \boldsymbol{I}_{K}\right)\right)\left(\left(\boldsymbol{I}_{K p_{2}}-\boldsymbol{\Pi}_{m}^{\prime} \boldsymbol{\Pi}_{m}\right) \otimes \boldsymbol{I}_{K}\right) \boldsymbol{\delta}
\end{aligned}
$$


CHAPTER 2

$$
\begin{gathered}
+O\left(T^{-1 / 2}\right)-\sqrt{T} \boldsymbol{\mu}\left(\boldsymbol{\theta}_{T, m}, \mathbf{0}_{K^{2}(p-m)}, \boldsymbol{\sigma}\right) \\
=\frac{\partial \boldsymbol{\mu}\left(\boldsymbol{\theta}_{\infty}, \boldsymbol{\sigma}\right)}{\partial \boldsymbol{\theta}^{\prime}}\left(\left[\boldsymbol{S}_{0}\left(\boldsymbol{I}_{K p_{2}}-\boldsymbol{\Pi}_{m}^{\prime} \boldsymbol{\Pi}_{m}\right)\right] \otimes \boldsymbol{I}_{K}\right) \boldsymbol{\delta}+O\left(T^{-1 / 2}\right) .
\end{gathered}
$$

The notation can be made a little lighter using the definitions of the Theorem: $\boldsymbol{D}_{\theta}=\partial \boldsymbol{\mu}\left(\boldsymbol{\theta}_{\infty}, \boldsymbol{\sigma}\right) / \partial \boldsymbol{\theta}^{\prime}$ and $\boldsymbol{D}_{\sigma}=\partial \boldsymbol{\mu}\left(\boldsymbol{\theta}_{\infty}, \boldsymbol{\sigma}\right) / \partial \boldsymbol{\sigma}^{\prime}$. Equations (2.25), (2.26) and (2.27) then combine to

$$
\begin{aligned}
\sqrt{T}\left(\boldsymbol{\mu}\left(\hat{\boldsymbol{\theta}}_{T, m}, \mathbf{0}_{K^{2}(p-m)}, \hat{\boldsymbol{\sigma}}\right)-\boldsymbol{\mu}\left(\boldsymbol{\theta}_{T, p}, \boldsymbol{\sigma}\right)\right) \\
\stackrel{d}{\longrightarrow} \boldsymbol{D}_{\theta}\left[\left(\boldsymbol{S}_{m} \otimes \boldsymbol{I}_{K}\right) \boldsymbol{A}_{m}-\left(\boldsymbol{S}_{0}\left(\boldsymbol{I}_{K p_{2}}-\boldsymbol{\Pi}_{m}^{\prime} \boldsymbol{\Pi}_{m}\right)\right) \otimes \boldsymbol{I}_{K}\right] \boldsymbol{\delta} \\
\quad+\boldsymbol{D}_{\theta}\left(\left(\boldsymbol{S}_{m}\left[\boldsymbol{S}_{m}^{\prime} \boldsymbol{\Omega}_{m} \boldsymbol{S}^{-1} \boldsymbol{S}_{m}^{\prime}\right) \otimes \boldsymbol{I}_{K}\right) \boldsymbol{R}+\boldsymbol{D}_{\sigma} \boldsymbol{S} .\right.
\end{aligned}
$$

With the definition of $\boldsymbol{A}_{m}$ we have

$$
\begin{aligned}
& \left(\boldsymbol{S}_{m} \otimes \boldsymbol{I}_{K}\right) \boldsymbol{A}_{m}-\left(\boldsymbol{S}_{0}\left(\boldsymbol{I}_{K p_{2}}-\boldsymbol{\Pi}_{m}^{\prime} \boldsymbol{\Pi}_{m}\right)\right) \otimes \boldsymbol{I}_{K} \\
= & \left(\boldsymbol{S}_{m} \otimes \boldsymbol{I}_{K}\right)\left(\left[\boldsymbol{S}_{m}^{\prime} \boldsymbol{\Omega} \boldsymbol{S}_{m}\right]^{-1} \boldsymbol{S}_{m}^{\prime} \boldsymbol{\Omega} \boldsymbol{S}_{0}\left(\boldsymbol{I}_{K p_{2}}-\boldsymbol{\Pi}_{m}^{\prime} \boldsymbol{\Pi}_{m}\right) \otimes \boldsymbol{I}_{K}\right) \\
& -\left(\boldsymbol{S}_{0}\left(\boldsymbol{I}_{K p_{2}}-\boldsymbol{\Pi}_{m}^{\prime} \boldsymbol{\Pi}_{m}\right)\right) \otimes \boldsymbol{I}_{K} \\
= & {\left.\left[\boldsymbol{S}_{m}\left[\boldsymbol{S}_{m}^{\prime} \boldsymbol{\Omega} \boldsymbol{S}_{m}\right]^{-1} \boldsymbol{S}_{m}^{\prime} \boldsymbol{\Omega} \boldsymbol{S}_{0}\left(\boldsymbol{I}_{K p_{2}}-\boldsymbol{\Pi}_{m}^{\prime} \boldsymbol{\Pi}_{m}\right)\right] \otimes \boldsymbol{I}_{K}\right)-\left(\boldsymbol{S}_{0}\left(\boldsymbol{I}_{K p_{2}}-\boldsymbol{\Pi}_{m}^{\prime} \boldsymbol{\Pi}_{m}\right)\right) \otimes \boldsymbol{I}_{K} } \\
= & {\left[\left(\boldsymbol{S}_{m}\left[\boldsymbol{S}_{m}^{\prime} \boldsymbol{\Omega} \boldsymbol{S}_{m}\right]^{-1} \boldsymbol{S}_{m}^{\prime} \boldsymbol{\Omega}-\boldsymbol{I}_{K p}\right) \boldsymbol{S}_{0}\left(\boldsymbol{I}_{K p_{2}}-\boldsymbol{\Pi}_{m}^{\prime} \boldsymbol{\Pi}_{m}\right)\right] \otimes \boldsymbol{I}_{K} . }
\end{aligned}
$$

Now, defining $\boldsymbol{P}_{m}:=\boldsymbol{S}_{m}\left[\boldsymbol{S}_{m}^{\prime} \boldsymbol{\Omega} \boldsymbol{S}_{m}\right]^{-1} \boldsymbol{S}_{m}^{\prime} \otimes \boldsymbol{I}_{K}$ and $\boldsymbol{C}_{m}:=\left(\boldsymbol{S}_{m}\left[\boldsymbol{S}_{m}^{\prime} \boldsymbol{\Omega} \boldsymbol{S}_{m}\right]^{-1} \boldsymbol{S}_{m}^{\prime} \boldsymbol{\Omega}-\right.$ $\left.\boldsymbol{I}_{K p}\right) \boldsymbol{S}_{0}\left(\boldsymbol{I}_{K p_{2}}-\boldsymbol{\Pi}_{m}^{\prime} \boldsymbol{\Pi}_{m}\right) \otimes \boldsymbol{I}_{K}$ we can write Equation (2.28) as

$$
\begin{gathered}
\sqrt{T}\left(\boldsymbol{\mu}\left(\hat{\boldsymbol{\theta}}_{T, m}, \mathbf{0}_{K^{2}(p-m)}, \hat{\boldsymbol{\sigma}}\right)-\boldsymbol{\mu}\left(\boldsymbol{\theta}_{T, p}, \boldsymbol{\sigma}\right)\right) \stackrel{d}{\longrightarrow} \boldsymbol{D}_{\theta} \boldsymbol{C}_{m} \boldsymbol{\delta}+\boldsymbol{D}_{\theta} \boldsymbol{P}_{m} \boldsymbol{R}+\boldsymbol{D}_{\sigma} \boldsymbol{S} \\
\sim \mathrm{N}\left(\boldsymbol{D}_{\theta} \boldsymbol{C}_{m} \boldsymbol{\delta}, \boldsymbol{D}_{\theta} \boldsymbol{P}_{m}(\boldsymbol{\Omega} \otimes \boldsymbol{\Sigma}) \boldsymbol{P}_{m} \boldsymbol{D}_{\theta}^{\prime}+\boldsymbol{D}_{\sigma} \boldsymbol{\Xi}_{22} \boldsymbol{D}_{\sigma}^{\prime}\right)
\end{gathered}
$$

what was to be shown.

Proof of Theorem 2.11. By Theorem 2.7

$$
\begin{aligned}
\sqrt{T}\left(\bar{\mu}(\boldsymbol{w})-\mu\left(\boldsymbol{\theta}_{T, p}, \boldsymbol{\sigma}\right)\right) & =\sum_{m=p_{1}}^{p} w_{m}\left[\sqrt{T}\left(\mu\left(\hat{\boldsymbol{\theta}}_{T, m}, \mathbf{0}_{K^{2}(p-m)}, \hat{\boldsymbol{\sigma}}\right)-\mu\left(\boldsymbol{\theta}_{T, p}, \boldsymbol{\sigma}\right)\right)\right] \\
& \stackrel{d}{\longrightarrow} \boldsymbol{D}_{\theta} \sum_{m=p_{1}}^{p} w_{m} \boldsymbol{C}_{m} \boldsymbol{\delta}+\boldsymbol{D}_{\theta} \sum_{m=p_{1}}^{p} w_{m} \boldsymbol{P}_{m} \boldsymbol{R}+\boldsymbol{D}_{\sigma} \boldsymbol{S} .
\end{aligned}
$$

The calculation of the mean vector and the asymptotic covariance matrix is straightforward.

Proof of Theorem 2.15. A valid confidence interval for a scalar focus was derived in Theorem 6 of Liu (2015). We follow the same reasoning. First, for the selection matrices of zeros and ones, we find

$$
\boldsymbol{I}_{K p}-\boldsymbol{S}_{0} \boldsymbol{S}_{0}^{\prime}+\boldsymbol{S}_{0} \boldsymbol{\Pi}_{m}^{\prime} \boldsymbol{\Pi}_{m} \boldsymbol{S}_{0}^{\prime}
$$


$=\boldsymbol{I}_{K p}-\left(\begin{array}{cc}\mathbf{O} & \mathbf{O} \\ \mathbf{O} & \boldsymbol{I}_{K p_{2}}\end{array}\right)+\left(\begin{array}{ccc}\mathbf{O}_{K p_{1} \times K p_{1}} & \mathbf{O} & \mathbf{O} \\ \mathbf{O} & \boldsymbol{I}_{K\left(m-p_{1}\right)} & \mathbf{O} \\ \mathbf{O} & \mathbf{O} & \mathbf{O}\end{array}\right)=\left(\begin{array}{cc}\boldsymbol{I}_{K m} & \mathbf{O} \\ \mathbf{O} & \mathbf{O}\end{array}\right)=\boldsymbol{S}_{m} \boldsymbol{S}_{m}^{\prime}$,

which we use to conclude

$$
\begin{aligned}
\boldsymbol{P}_{m} & -\boldsymbol{C}_{m}\left(\boldsymbol{S}_{0}^{\prime} \boldsymbol{\Omega}^{-1} \otimes \boldsymbol{I}_{K}\right) \\
& =\boldsymbol{S}_{m}\left[\boldsymbol{S}_{m}^{\prime} \boldsymbol{\Omega} \boldsymbol{S}_{m}\right]^{-1} \boldsymbol{S}_{m}^{\prime} \otimes \boldsymbol{I}_{K} \\
& -\left(\left(\boldsymbol{S}_{m}\left[\boldsymbol{S}_{m}^{\prime} \boldsymbol{\Omega} \boldsymbol{S}_{m}\right]^{-1} \boldsymbol{S}_{m}^{\prime} \boldsymbol{\Omega}-\boldsymbol{I}_{K p}\right) \boldsymbol{S}_{0}\left(\boldsymbol{I}_{K p_{2}}-\boldsymbol{\Pi}_{m}^{\prime} \boldsymbol{\Pi}_{m}\right) \otimes \boldsymbol{I}_{K}\right)\left(\boldsymbol{S}_{0}^{\prime} \boldsymbol{\Omega}^{-1} \otimes \boldsymbol{I}_{K}\right) \\
& =\left[\boldsymbol{S}_{m}\left[\boldsymbol{S}_{m}^{\prime} \boldsymbol{\Omega} \boldsymbol{S}_{m}\right]^{-1} \boldsymbol{S}_{m}^{\prime}\right. \\
& \left.-\left(\left(\boldsymbol{S}_{m}\left[\boldsymbol{S}_{m}^{\prime} \boldsymbol{\Omega} \boldsymbol{S}_{m}\right]^{-1} \boldsymbol{S}_{m}^{\prime} \boldsymbol{\Omega}-\boldsymbol{I}_{K p}\right) \boldsymbol{S}_{0}\left(\boldsymbol{I}_{K p_{2}}-\boldsymbol{\Pi}_{m}^{\prime} \boldsymbol{\Pi}_{m}\right)\right)\left(\boldsymbol{S}_{0}^{\prime} \boldsymbol{\Omega}^{-1}\right)\right] \otimes \boldsymbol{I}_{K} \\
& =\left[\boldsymbol{S}_{m}\left[\boldsymbol{S}_{m}^{\prime} \boldsymbol{\Omega} \boldsymbol{S}_{m}\right]^{-1} \boldsymbol{S}_{m}^{\prime} \boldsymbol{\Omega}\right. \\
& \left.-\left(\left(\boldsymbol{S}_{m}\left[\boldsymbol{S}_{m}^{\prime} \boldsymbol{\Omega} \boldsymbol{S}_{m}\right]^{-1} \boldsymbol{S}_{m}^{\prime} \boldsymbol{\Omega}-\boldsymbol{I}_{K p}\right) \boldsymbol{S}_{0}\left(\boldsymbol{I}_{K p_{2}}-\boldsymbol{\Pi}_{m}^{\prime} \boldsymbol{\Pi}_{m}\right)\right) \boldsymbol{S}_{0}^{\prime}\right] \boldsymbol{\Omega}^{-1} \otimes \boldsymbol{I}_{K} \\
& =\left[\boldsymbol{S}_{m}\left[\boldsymbol{S}_{m}^{\prime} \boldsymbol{\Omega} \boldsymbol{S}_{m}\right]^{-1} \boldsymbol{S}_{m}^{\prime} \boldsymbol{\Omega}\left(\boldsymbol{I}_{K p}-\boldsymbol{S}_{0}\left(\boldsymbol{I}_{K p_{2}}-\boldsymbol{\Pi}_{m}^{\prime} \boldsymbol{\Pi}_{m}\right) \boldsymbol{S}_{0}^{\prime}\right)\right. \\
& \left.+\boldsymbol{S}_{0}\left(\boldsymbol{I}_{K p_{2}}-\boldsymbol{\Pi}_{m}^{\prime} \boldsymbol{\Pi}_{m}\right) \boldsymbol{S}_{0}^{\prime}\right] \boldsymbol{\Omega}^{-1} \otimes \boldsymbol{I}_{K} \\
& =\left[\boldsymbol{S}_{m} \boldsymbol{S}_{m}^{\prime}+\boldsymbol{S}_{0}\left(\boldsymbol{I}_{K p_{2}}-\boldsymbol{\Pi}_{m}^{\prime} \boldsymbol{\Pi}_{m}\right) \boldsymbol{S}_{0}^{\prime}\right] \boldsymbol{\Omega}^{-1} \otimes \boldsymbol{I}_{K}=\boldsymbol{\Omega}^{-1} \otimes \boldsymbol{I}_{K} .
\end{aligned}
$$

So there is no dependence on model size $m$. Now, we use this expression and Theorem 2.11 to obtain

$$
\begin{aligned}
\sqrt{T}\left(\overline{\boldsymbol{\mu}}(\hat{\boldsymbol{w}})-\boldsymbol{\mu}\left(\boldsymbol{\theta}_{T, p}, \boldsymbol{\sigma}\right)\right) & \stackrel{d}{\longrightarrow} \boldsymbol{D}_{\theta} \sum_{m=p_{1}}^{p} w_{m}\left(\boldsymbol{R}_{\delta}\right) \boldsymbol{C}_{m} \boldsymbol{\delta}+\boldsymbol{D}_{\theta} \sum_{m=p_{1}}^{p} w_{m}\left(\boldsymbol{R}_{\delta}\right) \boldsymbol{P}_{m} \boldsymbol{R}+\boldsymbol{D}_{\sigma} \boldsymbol{S} \\
= & \boldsymbol{D}_{\theta} \sum_{m=p_{1}}^{p} w_{m}\left(\boldsymbol{R}_{\delta}\right) \boldsymbol{C}_{m}\left[\boldsymbol{\delta}+\left(\boldsymbol{S}_{0}^{\prime} \boldsymbol{\Omega}^{-1} \otimes \boldsymbol{I}_{K}\right) \boldsymbol{R}\right] \\
& +\boldsymbol{D}_{\theta} \sum_{m=p_{1}}^{p} w_{m}\left(\boldsymbol{R}_{\delta}\right)\left[\boldsymbol{P}_{m}-\boldsymbol{C}_{m}\left(\boldsymbol{S}_{0}^{\prime} \boldsymbol{\Omega}^{-1} \otimes \boldsymbol{I}_{K}\right)\right] \boldsymbol{R}+\boldsymbol{D}_{\sigma} \boldsymbol{S} \\
= & \boldsymbol{D}_{\theta} \sum_{m=p_{1}}^{p} w_{m}\left(\boldsymbol{R}_{\delta}\right) \boldsymbol{C}_{m} \boldsymbol{R}_{\delta}+\boldsymbol{D}_{\theta}\left(\boldsymbol{\Omega}^{-1} \otimes \boldsymbol{I}_{K}\right) \boldsymbol{R}+\boldsymbol{D}_{\sigma} \boldsymbol{S} .
\end{aligned}
$$

Next, by the convergence of $\hat{\boldsymbol{\delta}}$ to $\boldsymbol{R}_{\delta}$,

$$
\begin{array}{r}
\sqrt{T}\left(\overline{\boldsymbol{\mu}}(\hat{\boldsymbol{w}})-\boldsymbol{\mu}\left(\boldsymbol{\theta}_{T, p}, \boldsymbol{\sigma}\right)\right)-\boldsymbol{D}_{\theta} \sum_{m=p_{1}}^{p} \hat{w}_{m} \hat{\boldsymbol{C}}_{m} \hat{\boldsymbol{\delta}} \\
\stackrel{d}{\longrightarrow} \mathrm{N}\left(\mathbf{0}, \boldsymbol{D}_{\theta}\left(\boldsymbol{\Omega}^{-1} \otimes \boldsymbol{\Sigma}\right) \boldsymbol{D}_{\theta}^{\prime}+\boldsymbol{D}_{\sigma} \boldsymbol{\Xi}_{22} \boldsymbol{D}_{\sigma}^{\prime}\right) .
\end{array}
$$

The confidence region is constructed from the standardized quadratic form with population quantities replaced by their consistent estimates. 
Proof of Theorem 2.17 Consider $m \neq p$. We define $\boldsymbol{\alpha}_{m}=\boldsymbol{C}_{m}^{\prime} \boldsymbol{D}_{\theta}^{\prime}, \mathcal{A}=$ $\boldsymbol{S}_{0}^{\prime} \boldsymbol{\Omega}^{-1} \boldsymbol{S}_{0} \otimes \boldsymbol{\Sigma}$, and introduce a standard normally distributed random vector $\boldsymbol{Z}_{K^{2} p_{2}} \sim \mathrm{N}\left(\mathbf{0}, \boldsymbol{I}_{K^{2} p_{2}}\right)$ and random variable $Z \sim \mathrm{N}(0,1)$. Then $\boldsymbol{R}_{\delta}=\boldsymbol{\delta}+\mathcal{A}^{1 / 2} \boldsymbol{Z}_{K^{2} p_{2}}$, and

$$
\begin{aligned}
\boldsymbol{R}_{\delta}^{\prime} \boldsymbol{C}_{m}^{\prime} \boldsymbol{D}_{\theta}^{\prime} \boldsymbol{D}_{\theta} \boldsymbol{C}_{m} \boldsymbol{R}_{\delta} & =\left(\boldsymbol{\alpha}_{m}^{\prime}\left(\boldsymbol{\delta}+\mathcal{A}^{1 / 2} \boldsymbol{Z}_{K^{2} p_{2}}\right)\right)^{2} \\
& =\left\|\boldsymbol{\alpha}_{m}^{\prime} \mathcal{A}^{1 / 2}\right\|^{2}\left(\frac{\boldsymbol{\alpha}_{m}^{\prime} \mathcal{A}^{1 / 2} \boldsymbol{Z}_{K^{2} p_{2}}}{\left\|\boldsymbol{\alpha}_{m}^{\prime} \mathcal{A}^{1 / 2}\right\|}+\frac{\boldsymbol{\alpha}_{m}^{\prime} \boldsymbol{\delta}}{\left\|\boldsymbol{\alpha}_{m}^{\prime} \mathcal{A}^{1 / 2}\right\|}\right)^{2} \\
& =\left(\boldsymbol{\alpha}_{m}^{\prime} \mathcal{A} \boldsymbol{\alpha}_{m}\right)\left(Z+\boldsymbol{\alpha}_{m}^{\prime} \boldsymbol{\delta} / \sqrt{\boldsymbol{\alpha}_{m}^{\prime} \mathcal{A} \boldsymbol{\alpha}_{m}}\right)^{2} \\
& \sim a_{m} \chi_{\text {noncentral }}^{2}\left(1,\left(\boldsymbol{D}_{\theta} \boldsymbol{C}_{m} \boldsymbol{\delta}\right)^{2} / a_{m}\right)
\end{aligned}
$$

where $\boldsymbol{\alpha}_{m}^{\prime} \mathcal{A} \boldsymbol{\alpha}_{m}=a_{m}$. See Chapter 29 of Johnson et al. (1994) for details and moments. Finally, all quantities in $\widehat{F I C}_{p}=\boldsymbol{D}_{\theta}\left(\hat{\boldsymbol{\Omega}}^{-1} \otimes \hat{\boldsymbol{\Sigma}}\right) \boldsymbol{D}_{\theta}^{\prime}+\boldsymbol{D}_{\sigma} \boldsymbol{\Xi}_{22} \boldsymbol{D}_{\sigma}^{\prime}$ are estimated consistently.

Proof of Theorem 2.18 If $m=p$ and/or $l=p$, then there is no bias contribution and the matrix elements converge in probability. Now consider $m, l \neq p$, then (a) For $m=l$ the proof is identical to the proof of Theorem 2.17.

(b) Start by noting that for general $\boldsymbol{x}, \boldsymbol{x}^{\prime} \boldsymbol{A} \boldsymbol{x}=\boldsymbol{x}^{\prime}\left(\frac{\boldsymbol{A}+\boldsymbol{A}^{\prime}}{2}\right) \boldsymbol{x}$. Hence, with the definitions from the previous proof,

$$
\begin{aligned}
\boldsymbol{R}_{\delta}^{\prime} \boldsymbol{\alpha}_{m} \boldsymbol{\alpha}_{l}^{\prime} \boldsymbol{R}_{\delta} & =\left(\mathcal{A}^{-1 / 2} \boldsymbol{\delta}+\boldsymbol{Z}_{K^{2} p_{2}}\right)^{\prime}\left[\mathcal{A}^{1 / 2}\left(\frac{\boldsymbol{\alpha}_{m} \boldsymbol{\alpha}_{l}^{\prime}+\boldsymbol{\alpha}_{l} \boldsymbol{\alpha}_{m}^{\prime}}{2}\right) \mathcal{A}^{1 / 2}\right] \\
& \times\left(\mathcal{A}^{-1 / 2} \boldsymbol{\delta}+\boldsymbol{Z}_{K^{2} p_{2}}\right) .
\end{aligned}
$$

We subsequently use the transformation stated in Imhof (1961). The matrix in square brackets is symmetric and has a rank of at most two. The eigenvalue decomposition mentioned in Theorem 2.18 applies, and therefore

$$
\boldsymbol{R}_{\delta}^{\prime} \boldsymbol{\alpha}_{m} \boldsymbol{\alpha}_{l}^{\prime} \boldsymbol{R}_{\delta}=\sum_{i=1}^{2} \lambda_{i}\left(\boldsymbol{v}_{i}^{\prime} \mathcal{A}^{-1 / 2} \boldsymbol{\delta}+Z_{i}\right)^{2} \sim \sum_{i=1}^{2} \lambda_{i} \chi_{\text {noncentral }}^{2}\left(1,\left(\boldsymbol{v}_{i}^{\prime} \mathcal{A}^{-1 / 2} \boldsymbol{\delta}\right)^{2}\right)
$$

where the independence of the $Z_{i}$ follows from orthonormality of the eigenvectors. 


\section{Chapter 3}

\section{Consistency of Averaged Impulse Response Estimators in VARs}

We are concerned with model averaging estimators of impulse response coefficients for stationary VAR models of finite lag order, specifically the smoothed AIC- and BIC-based estimators (sAIC, sBIC). We show that there is not one unique way to define each of these estimators, but that instead there is a whole class of smoothed AIC and smoothed BIC estimators defined by a weight scaling factor, and we discuss its effect on the averaging weights. We extend results by Zhang (2015) for a time series setting by showing root- $T$ consistency of the estimators for specific ranges of the scaling factors. We then propose a bootstrap method to estimate the distributions of the averaging estimators. Using results of Bose (1988) we also show its asymptotic validity. In simulations we show the benefits of using sAIC-based instead of AIC-based estimators for estimating impulse response coefficients. ${ }^{1}$

\subsection{Introduction}

Model averaging is an alternative to model selection when there is model uncertainty. While in a Bayesian framework model averaging has been propagated and used already some time ago to address model uncertainty, the theory for frequentist settings is of more recent vintage, one important contribution of which is Hansen (2007). Instead of putting all weight on the estimates from one model, several models' parameter estimates are averaged. Hence, the number of possible estimates is increased: Not just the weight vectors $(1,0, \ldots, 0),(0,1,0, \ldots, 0), \ldots,(0, \ldots, 0,1)$ are allowed, but also all of their convex combinations, cf. Section 3.3 below and the definition of $\mathcal{H}_{n}$ in Liu (2015). This added freedom of model averaging can lead to better performance, e.g. lower MSE, compared to that of model selection

\footnotetext{
${ }^{1}$ The paper that this chapter is based on was presented at the 2017 SNDE conference in Paris.
} 


\section{CHAPTER 3}

estimators in finite samples. Based on a VAR process designed to mimic data used in Stock and Watson (2001) we give an example in Figure 3.13 below. Also for forecasting it is well known that combinations often outperform forecasts that are based on a single model, cf. Bates and Granger (1969).

Our contribution to theoretical econometrics of stationary time series is twofold. While it has been known that using consistent model selection criteria leads to consistent estimation of model parameters, we show that the averaged estimator is also consistent. Secondly, we show the validity of a bootstrap procedure for estimating the averaged estimator's distribution.

The averaged estimator is a weighted average over models of different orders with weights calculated as e.g. smoothed BIC weights (sBIC), cf. Buckland et al. (1997). We show that this estimator has the same asymptotic distribution as if the researcher only used the true order VAR model for estimation, provided a consistent criterion like BIC is used for calculating the weights. When using AIC for this, the averaged estimator is still root- $T$ consistent. It is not sufficient to show that most weight will be given to the correct model, which follows from the results for model selection. Additionally, we also need to deduce the rates of convergence of the model weights and show that these are $O_{p}\left(T^{-1 / 2}\right)$, where $T$ is the sample size. Otherwise the asymptotic bias of the small models ${ }^{2}$ would lead to a biased averaged estimator. Our results hold for multivariate, stationary, non-trending VARs, extending the work of Zhang (2015), who treats the case of a non dynamic linear regression model. Anderson (2003) and Paulsen (1984) prove to be useful for this and we adapted their proof techniques. We assume that the true lag order of the multivariate DGP is not known, but only an upper bound for it. The case of a $\operatorname{VAR}(\infty)$ where the estimated lag order is allowed to increase with sample size is not treated here. But our results could possibly also be extended to this setting using Jirak (2012), who proves consistency of BIC selection when the lag order of the autoregressive DGP is allowed to diverge with sample size.

We generalize the results of Zhang (2015) in another way by also treating functions of the model parameters, instead of only considering estimates of the model parameters themselves. This chapter is focused on estimation of impulse response coefficients, but the theoretical results still hold for other smooth functions of model parameters. To be precise, the proofs will hold for any object that can be expressed as a continuously differentiable function of the (estimates of the) autoregressive parameters $\boldsymbol{B}$ and covariance matrix $\boldsymbol{\Sigma}_{u}$ defined in Section 3.2 below. We have chosen impulse responses as an application because of their prominent role in macroeconomics. An important example of the study of impulse response functions is the seminal work of Stock and Watson (2001), on whose estimates we base a set of simulations. We consider orthogonalized impulse responses based on the Choleski decomposition of the estimated covariance matrix, cf. Chapter 1 for a discussion of this choice, to not overly complicate this part of the analysis and concentrate the discussion on our main contributions of showing estimator consistency and bootstrap validity. This identification scheme is also used by Lütkepohl et al. (2015).

\footnotetext{
${ }^{2}$ In our setting we call those models (too) small which do not include the true DGP's lag order.
} 
Hansen (2005) makes the case that it might not be optimal to use the same model for estimation across different horizons of impulse responses. He presents evidence that for the impulse response at horizon 2 a different lag order may minimize the estimator's MSE than for the impulse response at horizon 6 . His results are obtained for the case of an $\operatorname{ARMA}(1,1)$ DGP that is being approximated by AR models. We allow for using different weights for different horizons. All of our results are conditional on the choice of one specific impulse response coefficient, so the response of one variable to an impulse in another after a certain number of periods. With regard to applied econometrics we make a direct comparison between the AIC-based and the sAIC-based estimator in a simulation study, and find that in terms of the estimator's mean squared error (MSE) the sAIC-based estimator often performs better than its selection counterpart (in finite samples). At the same time we show that the sAIC-based estimator retains the corresponding property of AIC selection of putting no weight on models, that are too small, asymptotically. This is an important message for practitioners: By using sAIC instead of AIC, estimation accuracy may improve while the asymptotic properties of AIC are still retained.

Our second contribution is the proposal of a bootstrap procedure for estimating the distribution of the averaged estimator and showing its asymptotic validity. The proof of asymptotic validity relies on the rates of convergence derived in the first part of the chapter, and on Bose (1988) and Kilian (1998b). Indeed, in our simulation study we find the effective coverages of the estimated confidence intervals from the selection and averaging methods to be similar, but in most cases those of the selection based estimator are closer to the nominal coverage level. The averaging-based effective coverages tend to be lower than the selection-based coverages. Hence, they might be valuable in settings where other methods tend to have effective coverages which are too large. We calculate joint confidence bands with a method suggested by Lütkepohl et al. (2015), who use AIC-based selection in a VAR setting to compare different methods for obtaining joint confidence bands for impulse response functions. Kilian (2001) compares the effective coverages of AIC, BIC and Hannan-Quinn model selection estimators of impulse response coefficients. He finds that the coverage of the BIC method is rather poor, an observation that we find still to hold in the averaging case.

Parts of the model averaging literature assume a local misspecification framework. Hjort and Claeskens (2003a)'s Theorem 4.1 shows that within that framework the asymptotic distribution of the estimator will generally be a mixture of normals with a nonzero bias term. We do not assume this type of local misspecification, just like the following texts, in which properties of model averaging estimators have been discussed. In Section 4.8 of Burnham and Anderson (2002) an sAIC-based averaged estimator is used for prediction, which the authors suggest to use instead of model selection when model uncertainty is large. Hansen and Racine (2012) use jackknife weights for averaging across OLS estimators in a regression setting, and show this method to outperform model selection methods. Liang et al. (2011) consider averaging estimators, based on sAIC and sBIC, among others, for a linear regression model. Hansen (2007)'s simulations show that using sAIC yields lower risk (a lower mean squared error (MSE)) than AIC for esti- 


\section{CHAPTER 3}

mating regression parameters. In a dependent data setting Zhang et al. (2013) also document that model averaging methods yield a lower MSE than selection methods, even though they do not compare AIC to its smoothed counterpart sAIC directly.

The chapter is organized as follows. Section 3.2 fixes notation and assumptions. Section 3.3 discusses consistency of the averaged estimator. Section 3.4 introduces the bootstrap procedure, and shows its first order accuracy. Section 3.5 provides simulation evidence on the performance of our methods. Section 3.6 concludes. An appendix contains the proofs.

\subsection{Model Setting}

We are concerned with VAR models for the $d$-dimensional variable $\boldsymbol{y}_{t}$ observed at $t=1, \ldots, T$, with $T$ the sample size, of the form

$$
\boldsymbol{y}_{t}=\boldsymbol{A}_{1} \boldsymbol{y}_{t-1}+\boldsymbol{A}_{2} \boldsymbol{y}_{t-2}+\ldots+\boldsymbol{A}_{\bar{p}} \boldsymbol{y}_{t-\bar{p}}+\boldsymbol{u}_{t} .
$$

We assume that a fixed but unknown $p_{0}, p_{0} \leq \bar{p}$ is the lag order of the true DGP. So $\boldsymbol{A}_{p_{0}} \neq \mathbf{O}, \boldsymbol{A}_{p_{0}+1}, \ldots, \boldsymbol{A}_{\bar{p}}=\mathbf{O}$. Here $\mathbf{O}$ is a matrix of conformable dimension with only zeros. In later occurrences, when there is risk of confusion, we will add a subscript indicating the dimension, i.e. write $\mathbf{O}_{d}$. We further assume that the researcher only knows $\bar{p}$. She will average estimates across all models $p \in\{1, \ldots, \bar{p}\}$ by assigning weights to the estimates from these different models. ${ }^{3}$

For the DGP we assume $\boldsymbol{u}_{t} \sim$ iid $\left(\mathbf{0}, \boldsymbol{\Sigma}_{\boldsymbol{u}}\right), \boldsymbol{\Sigma}_{\boldsymbol{u}}$ to be positive definite, and $\boldsymbol{u}_{1}$ to have finite moments up to the eighth order. Furthermore, each element of $\left(\boldsymbol{u}_{1}, \boldsymbol{u}_{1} \boldsymbol{u}_{1}^{\prime}\right)$, which is of dimension $(d, d+1)$, satisfies Cramér's condition. As an example consider the case $d=2, \boldsymbol{u}_{1}=(v, w)^{\prime}$. Then for the vector $(v, v w)$ Cramér's condition on its characteristic function reads: For every $k>0$, there exists a $\delta>0$ such that

$$
\sup _{l \in \mathbb{R}^{2}:\|l\|>k}\left|E \exp \left(i l^{\prime}(v, v w)^{\prime}\right)\right| \leq \exp (-\delta)
$$

with $i$ denoting the imaginary unit. Compare Chapter 2 of Hall (1992) for a detailed discussion of Cramér's condition. We also assume stability of the process:

$$
\operatorname{det}\left(\boldsymbol{I}_{d}-\boldsymbol{A}_{1} z-\ldots-\boldsymbol{A}_{p_{0}} z^{p_{0}}\right) \neq 0 \quad \text { for }|z| \leq 1 .
$$

Here $\boldsymbol{I}_{d}$ is the $d$-dimensional identity matrix. This implies weak stationarity. This setting is the one of Kilian (1998b) and it is also covered by Bose (1988).

Note that we will show that the weights for larger models, so with $p>p_{0}$, will tend to 0 asymptotically, which is why most of the following notation will be given in terms of $p_{0}$ only. Analogously to Lütkepohl (2005) we define

$$
\boldsymbol{Y}=\left(\boldsymbol{y}_{1}, \ldots, \boldsymbol{y}_{T}\right),
$$

\footnotetext{
${ }^{3}$ We discuss a practical method that has been suggested for choosing $\bar{p}$ large enough in practical applications in Section 3.5.
} 


$$
\begin{array}{rlrl}
\boldsymbol{B} & =\left(\boldsymbol{A}_{1}, \ldots, \boldsymbol{A}_{p_{0}}\right), & \boldsymbol{\beta}=\operatorname{vec}(\boldsymbol{B}), \\
\boldsymbol{Z}_{t}=\left(\begin{array}{c}
\boldsymbol{y}_{t} \\
\boldsymbol{y}_{t-1} \\
\vdots \\
\boldsymbol{y}_{t-p_{0}+1}
\end{array}\right), & \boldsymbol{Z}=\left(\boldsymbol{Z}_{0}, \ldots, \boldsymbol{Z}_{T-1}\right), \\
\boldsymbol{U}=\left(\boldsymbol{u}_{1}, \ldots, \boldsymbol{u}_{T}\right), & \boldsymbol{\sigma}=\operatorname{vech}\left(\boldsymbol{\Sigma}_{\boldsymbol{u}}\right) .
\end{array}
$$

This is the standard VAR model often used as a benchmark in macroeconomics, cf. Christiano et al. (1999), Benkwitz et al. (2000), Lütkepohl et al. (2015). As the workings of this model are well researched we use it as a basis for our analysis. For the next section, which discusses the definition of the averaging weights and gives the main results of this chapter, we also need the following observation, which is a direct consequence of the stationarity of the process together with Lemma 3.1 of Lütkepohl (2005):

$$
T^{-1} \boldsymbol{Z} \boldsymbol{Z}^{\prime} \stackrel{p}{\longrightarrow} \boldsymbol{\Omega}, \quad \text { and } \quad T^{-1 / 2} \operatorname{vec}\left(\boldsymbol{U} \boldsymbol{Z}^{\prime}\right) \stackrel{d}{\longrightarrow} \mathcal{N}\left(\mathbf{0}, \boldsymbol{\Omega} \otimes \boldsymbol{\Sigma}_{\boldsymbol{u}}\right)
$$

hold with $\boldsymbol{\Omega}$ positive definite. So it follows that the estimate $\widehat{\boldsymbol{B}}$ of $\boldsymbol{B}$ will be asymptotically normally distributed.

Our objects of interest are the orthogonalized impulse responses of the system. The responses of the $\operatorname{VAR}\left(p_{0}\right)$ system after $h \geq 1$ periods to reduced form disturbances are described by the recursion

$$
\boldsymbol{\Phi}_{h}=\sum_{j=1}^{h} \boldsymbol{\Phi}_{h-j} \boldsymbol{A}_{j}, \text { where } \boldsymbol{\Phi}_{0}=\boldsymbol{I}_{d}, \boldsymbol{A}_{j}=\mathbf{O}_{d} \text { for } j>p_{0}
$$

Let $\boldsymbol{P}$ be the lower triangular matrix from the Cholesky decomposition $\boldsymbol{\Sigma}_{\boldsymbol{u}}=\boldsymbol{P} \boldsymbol{P}^{\prime}$. The orthogonalized impulse responses are then defined as

$$
\Theta_{h}=\boldsymbol{\Phi}_{h} \boldsymbol{P} .
$$

We see that these are nonlinear functions of the coefficient matrices and the innovation covariance matrix, as we consider the recursive identification scheme based on the Cholesky decomposition of the latter. To be precise, in this chapter we will be interested in one particular impulse response coefficient, that is for given $i, j, h$, where $1 \leq i, j \leq d, h \geq 1$ we will be interested only in the $(i, j)$ element of the $\boldsymbol{\Theta}_{h}$ matrix. For example $\left(\boldsymbol{\Theta}_{3}\right)_{1,2}$ is the response of variable 1 to a shock in variable 2 which occurred 3 periods ago. Because all of our results are conditional on the choice of $i, j, h$ we will omit this in the notation and denote this object of interest by $\theta \in \mathbb{R}$.

Instead of selecting one model we take averages over estimators from different models. First, for every $p \in\{1, \ldots, \bar{p}\}$ the researcher obtains an estimate $\widehat{\theta}_{p}$ of $\theta$ as follows. OLS estimates $\widehat{\boldsymbol{A}}_{1}, \ldots, \widehat{\boldsymbol{A}}_{p}$ and an estimate $\widehat{\boldsymbol{\Sigma}}_{p}$ of the residual covariance matrix are calculated. Using Equations (3.3), (3.4), only replacing $p_{0}$ by $p$ and unknown terms by their estimates, $\widehat{\theta}_{p}$ is then calculated. Then, given an 


\section{CHAPTER 3}

estimated weight vector $\widehat{\boldsymbol{w}}_{x I C}=\left(\widehat{w}_{x I C, 1}, \ldots, \widehat{w}_{x I C, \bar{p}}\right)$, where xIC stands for AIC or BIC, respectively, we define the averaged estimator

$$
\widehat{\theta}\left(\widehat{\boldsymbol{w}}_{x I C}\right)=\widehat{w}_{x I C, 1} \widehat{\theta}_{1}+\widehat{w}_{x I C, 2} \widehat{\theta}_{2}+\ldots+\widehat{w}_{x I C, \bar{p}} \widehat{\theta}_{\bar{p}}
$$

In the next section the calculations of the weights will be detailed.

\subsection{Consistency of the Averaged Estimator}

Zhang (2015) shows consistency of the AIC- and BIC-based model average estimators in a linear regression setting. We generalize this result for sAIC and sBIC to the case of vector autoregressions and averages of estimated impulse response coefficients. For this we use methods of Paulsen (1984) and Anderson (2003). For BIC-based averaging, since the BIC is a strongly consistent criterion, we will even show $\widehat{w}_{B I C, p_{0}} \rightarrow 1$. So asymptotically only the true model will receive a positive weight.

In the $p$ th candidate model, $p \in\{1, \ldots, \bar{p}\}$, the AIC, BIC score, and smoothed weights are

$$
\begin{aligned}
A I C_{p} & =T \log \left|\widehat{\boldsymbol{\Sigma}}_{p}\right|+2 p d^{2}, \\
B I C_{p} & =T \log \left|\widehat{\boldsymbol{\Sigma}}_{p}\right|+p d^{2} \log (T), \\
\widehat{w}_{x I C, p} & =\exp \left(-x I C_{p} / 2\right) / \sum_{p=1}^{\bar{p}} \exp \left(-x I C_{p} / 2\right),
\end{aligned}
$$

where here and in the following xIC stands for $\mathrm{AIC}$ or BIC, respectively. This weights definition might be considered the standard case. It was suggested by Buckland et al. (1997) in connection with the Bayes factor approximation of Schwarz (1978) and has been used much since, among others in Zhang (2015), and Claeskens and Hjort (2008).

For computational implementation it is advisable to subtract $\min _{p \in\{1, \ldots, \bar{p}\}} A I C_{p}$ from the AIC scores, and the analogous number from the BIC scores, before calculating the weights to avoid exceeding the computationally allowed number sizes, cf. also Claeskens and Hjort (2008), Example 7.1. This transformation leaves the weights unchanged. Another commonly used definition of the BIC can be obtained by dividing the one given here by $T$. This scaling would not change anything in a model selection setting. For our case of averaging, however, the estimated weights would then converge to equal weights, $\widehat{w}_{x I C, p} \rightarrow 1 / \bar{p}$, cf. 3.3 .1 below.

Now note the following observations, which are the main ingredients to proving the results in this section.

$$
\begin{aligned}
& \exp \left(-A I C_{p} / 2\right)=\exp \left(-\frac{T}{2} \log \left|\widehat{\boldsymbol{\Sigma}}_{p}\right|\right) \exp \left(-p d^{2}\right)=\left|\widehat{\boldsymbol{\Sigma}}_{p}\right|^{-T / 2} \exp \left(-p d^{2}\right), \\
& \exp \left(-B I C_{p} / 2\right)=\exp \left(-\frac{T}{2} \log \left|\widehat{\boldsymbol{\Sigma}}_{p}\right|\right) \exp \left(-\frac{p d^{2}}{2} \log (T)\right)=\left|\widehat{\boldsymbol{\Sigma}}_{p}\right|^{-T / 2} T^{-p d^{2} / 2},
\end{aligned}
$$


and the weights are from the unit interval,

$$
\widehat{w}_{x I C, p} \in[0,1], \quad 1 \leq p \leq \bar{p},
$$

and sum to 1. From the following Lemma and Eqn (3.7) it follows that only model $p_{0}$ will receive positive $\mathrm{sBIC}$ weight in the limit, $\widehat{w}_{B I C, p_{0}} \stackrel{p}{\longrightarrow} 1$.

Lemma 3.1. Under the assumptions of Section 3.2 the following stochastic bounds hold for the estimated weights:

$$
\begin{array}{ll}
\widehat{w}_{B I C, p}=O_{p}\left(c_{1}^{T} T^{\left(p_{0}-p\right) d^{2} / 2}\right), & \text { for } p<p_{0}, \\
\widehat{w}_{B I C, p}=O_{p}\left(T^{\left(p_{0}-p\right) d^{2} / 2}\right), & \\
\widehat{w}_{A I C, p}=O_{p}\left(c_{2}^{T}\right), & \text { for } p>p_{0},
\end{array}
$$

where $c_{1}, c_{2} \in(0,1)$ are some generic constants.

Note that the last equation corresponds to the statement of Paulsen and Tjøstheim (1985) for model selection:

$$
\lim _{T \rightarrow \infty} P\left(\widehat{p}_{A I C}<p_{0}\right)=0
$$

This result is used by Kilian (1998a) to show bootstrap validity. Also like in model selection, the smoothed AIC estimator is not consistent. Even in the limit positive weight may be given to models that are too large. This, however, does not induce a bias, it only costs efficiency, because the estimates of the coefficients larger than $p_{0}$ converge to 0 . Even though small models yield a biased estimate, scaling by $\sqrt{T}$ does not destroy consistency, because the small models' weights tend to 0 fast enough. We state this as a theorem.

Theorem 3.2. Under the assumptions of Section 3.2,

$$
\begin{aligned}
\sqrt{T}\left(\widehat{\theta}\left(\widehat{\boldsymbol{w}}_{B I C}\right)-\theta\right) & =\sqrt{T}\left(\widehat{\theta}_{p_{0}}-\theta\right)+O_{p}\left(T^{-1 / 2}\right), \\
\sqrt{T}\left(\widehat{\theta}\left(\widehat{\boldsymbol{w}}_{A I C}\right)-\theta\right) & =O_{p}(1) .
\end{aligned}
$$

The result for the BIC averaged estimator is stronger in the sense that it gives the limiting distribution of the averaged estimator. It is stronger, because in our setting BIC is a consistent criterion, while AIC is not. Zhang (2015) has shown analogous results in a regression setting, but only for the parameter estimates of the regression, not for functions thereof, as in our case. (Note that he defines $\theta$ differently.) Our proof is a generalization of his ideas.

\subsubsection{Weight Scaling}

The smoothed model averaging estimators, sAIC, sBIC, discussed above are actually representatives of a whole class of averaging methods. We use this section to elaborate on this point, which has been raised for example in Chapter 7.2 of 


\section{CHAPTER 3}

Claeskens and Hjort (2008), and in Liang et al. (2011), but is otherwise often not mentioned when smoothed averaging estimators are being used.

Let $\kappa>0$ be a parameter. Above we defined the AIC score as

$$
A I C_{p}=\frac{T}{\kappa} \log \left|\widehat{\boldsymbol{\Sigma}}_{p}\right|+\frac{2}{\kappa} p d^{2}
$$

with $\kappa=1$. This is a commonly used definition, see e.g. Claeskens and Hjort (2008), Zhang (2015). It is also not uncommon to find the definition written down with $\kappa$ equal to $T$, the sample size, see e.g. Juselius (2006), Kilian (2001). For model selection the choice of $\kappa$ obviously is unimportant in finite samples, since it does not influence the ranking of the models. For model averaging, however, the choice of $\kappa$ becomes relevant, if, as above, we define model weights as

$$
\widehat{w}_{x I C, p}=\exp \left(-x I C_{p} / 2\right) / \sum_{p=1}^{\bar{p}} \exp \left(-x I C_{p} / 2\right) .
$$

(Note that adding or subtracting constants to/from the expression of the xIC score do not influence either the selected model nor the averaging weights.) Claeskens and Hjort (2008) pointed out that for small values of $\kappa$ the weights are more concentrated on few models, with the averaging estimator becoming more similar to the model selection estimator for $\kappa$ approaching 0 . For diverging values of $\kappa$ the weights become more spread out, the estimator becomes more similar to the equal weights estimator. In a static regression framework Liang et al. (2011) develop a method for choosing a set of parameters ( $\kappa$ and other parameters) that minimizes a certain risk quantity that is based on estimated MSE. As far as consistency of the smoothed averaging estimators as described by Theorem 3.2 above is concerned, we find (cf. Section 3.A.3) that it still holds as long as $\kappa \leq d^{2}$, with $d$ the number of variables of the VAR. For the simulations of the following section we stick to the original definition of the smoothed AIC (and smoothed BIC) as given above, so with $\kappa=1$, which was introduced by Buckland et al. (1997). We do this because this definition is most often used in the literature to date, and the results may thus be more interesting for practitioners.

\subsection{Bootstrapping}

To estimate the distribution of the averaged estimator we propose to use a bootstrap procedure. The necessity of having such a procedure available is illustrated by Figure 3.14, which is discussed in Section 3.5. For our setting the sieve bootstrap is usually preferred over block bootstrap. Lütkepohl et al. (2015), for example, find that among different methods of estimating confidence intervals the sieve-based bootstrap methods usually perform well. The procedure we suggest is based on the sieve bootstrap and similar to the one given in Kilian (1998a). We present two different versions, one with endogenous, and another one with exogenous calculations of weights, referring to the cases of the averaging weights being calculated for each bootstrap replication separately or together with estimating 
the lag order for bootstrap sample generation. Kilian (1998a) made the point that the coverage of the former is expected to be better for the case of model selection. As model selection and averaging criterion either AIC or BIC may be used. We write $\mathrm{xIC}$ as a placeholder. Assume the setting defined above: The DGP is of order $p_{0}$ with $p_{0} \leq \bar{p}$.

\section{Endogenous Weights Algorithm:}

1. Fit $\operatorname{VAR}(p)$ models, $1 \leq p \leq \bar{p}$ to the data $\left\{\boldsymbol{y}_{t}\right\}_{t=1}^{T}$,

$$
\boldsymbol{y}_{t}=\widehat{\boldsymbol{A}}_{1} \boldsymbol{y}_{t-1}+\widehat{\boldsymbol{A}}_{2} \boldsymbol{y}_{t-2}+\ldots+\widehat{\boldsymbol{A}}_{p} \boldsymbol{y}_{t-p}+\widehat{\boldsymbol{u}}_{t} .
$$

Determine $\widehat{p}$ by the xIC.

2. Generate bootstrap replications $\left\{\boldsymbol{y}_{t}^{*}\right\}_{t=1}^{T}$ based on the recursion

$$
\boldsymbol{y}_{t}^{*}=\widehat{\boldsymbol{A}}_{1} \boldsymbol{y}_{t-1}^{*}+\widehat{\boldsymbol{A}}_{2} \boldsymbol{y}_{t-2}^{*}+\ldots+\widehat{\boldsymbol{A}}_{\widehat{p}} \boldsymbol{y}_{t-\widehat{p}}^{*}+\boldsymbol{u}_{t}^{*},
$$

where the $\left\{\boldsymbol{u}_{t}^{*}\right\}_{t=1}^{T}$ are obtained as follows: Draw randomly with replacement from the $\widehat{\boldsymbol{u}}_{t}$ multiplied by $\left(\frac{T}{T-d \bar{p}-1}\right)^{1 / 2}$ (cf. Davidson and MacKinnon (1999)). Demean the set of $T$ draws.

For each sample of bootstrap replications $\left\{\boldsymbol{y}_{t}^{*}\right\}_{t=1}^{T}$

3. Fit $\operatorname{VAR}(p)$ models, $1 \leq p \leq \bar{p}$ to the series, calculate corresponding impulse response estimates $\widehat{\theta}_{1}^{*}, \ldots, \widehat{\theta}_{\bar{p}}^{*}$, and bootstrap weights $\widehat{\boldsymbol{w}}_{x I C}^{*}=$ $\left(\widehat{w}_{x I C, 1}^{*}, \ldots, \widehat{w}_{x I C, \bar{p}}^{*}\right)$.

4. Calculate the averaged impulse response coefficient

$$
\widehat{\theta}^{*}\left(\widehat{\boldsymbol{w}}_{x I C}^{*}\right)=\widehat{w}_{x I C, 1}^{*} \widehat{\theta}_{1}^{*}+\widehat{w}_{x I C, 2}^{*} \widehat{\theta}_{2}^{*}+\ldots+\widehat{w}_{x I C, \bar{p}}^{*} \widehat{\theta}_{\bar{p}}^{*} .
$$

Note that $\widehat{\theta}^{*}\left(\widehat{\boldsymbol{w}}_{x I C}^{*}\right)$ depends on the estimated weights as well as on the estimates for the VAR coefficient matrices and the innovation covariance matrix from all considered models. We can then obtain confidence bands for a nominal coverage of $1-\alpha$ by reading off the $\alpha / 2$ and $1-\alpha / 2$ percentile interval endpoints of the distribution of the bootstrap impulse response estimates $\widehat{\theta}^{*}\left(\widehat{\boldsymbol{w}}_{x I C}^{*}\right)$. Asympotic validity of the procedure is proven in Appendix 3.A.5.

There are important variations of this boostrap algorithm. These are also asymptotically valid: For the exogenous weights algorithm the weights are calculated in Step 1 instead of in Step 3 and kept fixed across all bootstrap replications. The endogenous weights algorithm is designed to take the weights' estimation uncertainty into account and will be our algorithm of choice in this chapter. We use it for our simulations. A case for endogenizing the estimation uncertainty in the algorithm in this way is made in Kilian (1998a) in the setting of model selection.

Secondly, instead of selecting a lag order based on information criteria, one could simply estimate a $\operatorname{VAR}(\bar{p})$, and use this to generate bootstrapped data. 


\section{CHAPTER 3}

Analogously to the proof of asymptotic validity given in the appendix for the AIC case, this method can also be shown to be valid. In a separate set of simulations, whose results are not given in this chapter, we found that the conclusions for coverages of bootstrapped estimates did not differ between using AIC or $\bar{p}$ for generating the bootstrap samples. Thirdly, Kilian (1998b) suggests to bias correct the $\boldsymbol{\beta}$ estimates based on expressions by Pope (1990). Since the bias estimate is of order $T^{-1}$ validity then still holds.

\subsection{Simulation Study}

In order to analyze the finite sample performance of the averaged estimators we carry out simulations in this section. We show results for two different simulation settings. To enable a direct comparison between results for model selection and our suggested model averaging procedure we apply the averaging estimators to the simulation setting that Kilian (2001) used for gauging the performance of model selection estimators. For a second set of simulations we also give results inspired by Stock and Watson (2001), which is a prototypical representative of the literature on monetary structural VARs. These two settings are treated in separate subsections in the following. We provide evidence for the theory developed in the previous section by showing the selection frequencies for each criterion and lag order, provide mean squared error, MSE, comparisons between the model selection and model averaging estimators, and give results for the effective coverages of the constructed confidence intervals. In Chapter 4 we apply our methods to a wider range of empirical macroeconomic data sets.

We will refer to the strategy that assumes the true lag order as known as the true lag order method: The researcher always estimates a model with the true lag order, which in the case of the data simulated by Kilian (2001) is a VAR(4). We calculate the estimators' MSE's as the sum of squared differences to the true impulse response (IR) function, which is calculated based on the true DGP, so based on the true VAR coefficients and covariance matrix.

The range of models that are considered for selection and averaging is defined by a maximum lag order, $\bar{p}$. In applied work it is common to let such a maximum lag increase with sample size, cf. Lütkepohl et al. (2015) who also posit a finite order DGP. In the infinite order setting of Ing and Wei (2005) $\bar{p}$ is allowed to increase at a rate of $\sqrt{T}$. To be precise, in their simulation setting they choose $\bar{p}$ as the largest integer smaller than $\sqrt{T}$. This approach seems to have been taken by Kilian (2001) as well who sets $\bar{p}=8$ for $T=80$, and we will do the same unless otherwise noted, setting $\bar{p}=12$ for $T=160$ for example. Note that the results of the following subsection are rather robust to the choice of $\bar{p}$. When we set $\bar{p}=8$ for $T=160$ the results in Tables 3.1, 3.2, and Figure 3.3 remained virtually unchanged. 


\subsubsection{Simulation Setting of Kilian (2001)}

To judge the finite sample performance of the averaged estimators we simulate data from a VAR(4) used in Kilian (2001), with coefficient matrices

$$
\begin{array}{lll}
\boldsymbol{A}_{1}=\left(\begin{array}{cc}
0.6362 & -0.0012 \\
0.0190 & 0.5782
\end{array}\right), & \boldsymbol{A}_{2}=\left(\begin{array}{cc}
-0.0168 & -0.0285 \\
0.5211 & -0.3041
\end{array}\right) \\
\boldsymbol{A}_{3}=\left(\begin{array}{cc}
0.0273 & -0.0028 \\
0.1568 & 0.2229
\end{array}\right), & \boldsymbol{A}_{4}=\left(\begin{array}{cc}
0.1517 & -0.0198 \\
-0.7600 & -0.3168
\end{array}\right) .
\end{array}
$$

The largest root of this process is 0.8894 . The innovation covariance matrix is $\boldsymbol{\Sigma}_{\boldsymbol{u}}=\left(\begin{array}{ll}0.025 & 0.009 \\ 0.009 & 0.387\end{array}\right) \times 10^{-3}$. We always plot the four impulse response functions up to horizon 16. As in Kilian (2001) the number of simulations is $N_{\text {sim }}=1000$, and number of bootstrap replications 1000. We apply the small sample bias correction to the OLS estimates of the parameters in $\boldsymbol{\beta}$ as described in the appendix of Kilian (2001). This particular process is designed to have impulse response functions similar to those "often encountered in applied work". These are plotted in Figure 3.1 together with the mean estimated impulse response functions of the model with the true lag order, the VAR(4), for 1000 simulations.

\section{Selection Frequencies, Average Weights, and MSE}

Table 1 in Kilian (2001) shows how the true lag order gets selected more frequently with sample size increasing from $T=80$ to $T=160$. Tables 3.1 and 3.2 below amend this information with average smoothed weights for sAIC and sBIC averaging. For every $p=1, \ldots, \bar{p}$ the selection frequency $N_{\text {sim }}^{-1} \sum_{i=1}^{N_{\text {sim }}} \mathbb{1}\{\widehat{p}(i)=p\}$, and average weight $N_{\text {sim }}^{-1} \sum_{i=1}^{N_{s i m}} \widehat{w}_{p}(i)$ in percentages are reported. Here $\widehat{p}(i), \widehat{w}_{p}(i)$ are the estimated lag order and estimated weights in simulation repetition $i$, respectively. So the row sums in the table add up to 100 (approximately only because of rounding imprecisions). For $T=80$ the maximum lag is $\bar{p}=8$, as explained above, and for larger sample sizes the weights and selection frequencies for models beyond lag 9 are smaller than $1 \%$.

As predicted by the theory of Lemma 3.1, for large sample sizes sBIC will assign all the weight to the true model, and sAIC will not assign any weight to models that are too small. For our simulated process even the rates of convergence towards zero correspond to the rates predicted in Lemma 3.1. The theoretical rate for the decrease of sBIC weights of large models is hyperbolic. And indeed, for the smallest shown sample size of $T=80$ already the average weight given to these models is zero. The second fastest rate is the exponential rate of decline of the sAIC weights for small models. These become zero at a sample size of $T=200$. Lastly, the mixed bound of the weights given to small models by sBIC consists of a hyperbolically increasing and an exponentially decreasing term, and it makes these weights zero from a sample size as small as $T=400$ already, hinting at the asymptotic property of consistency already at a relatively small sample size.

Interestingly, the values for sBIC average weights and BIC selection frequencies are more similar than the AIC counterparts. For example, $33 \%$ of the time $p=4$ 


\section{CHAPTER 3}
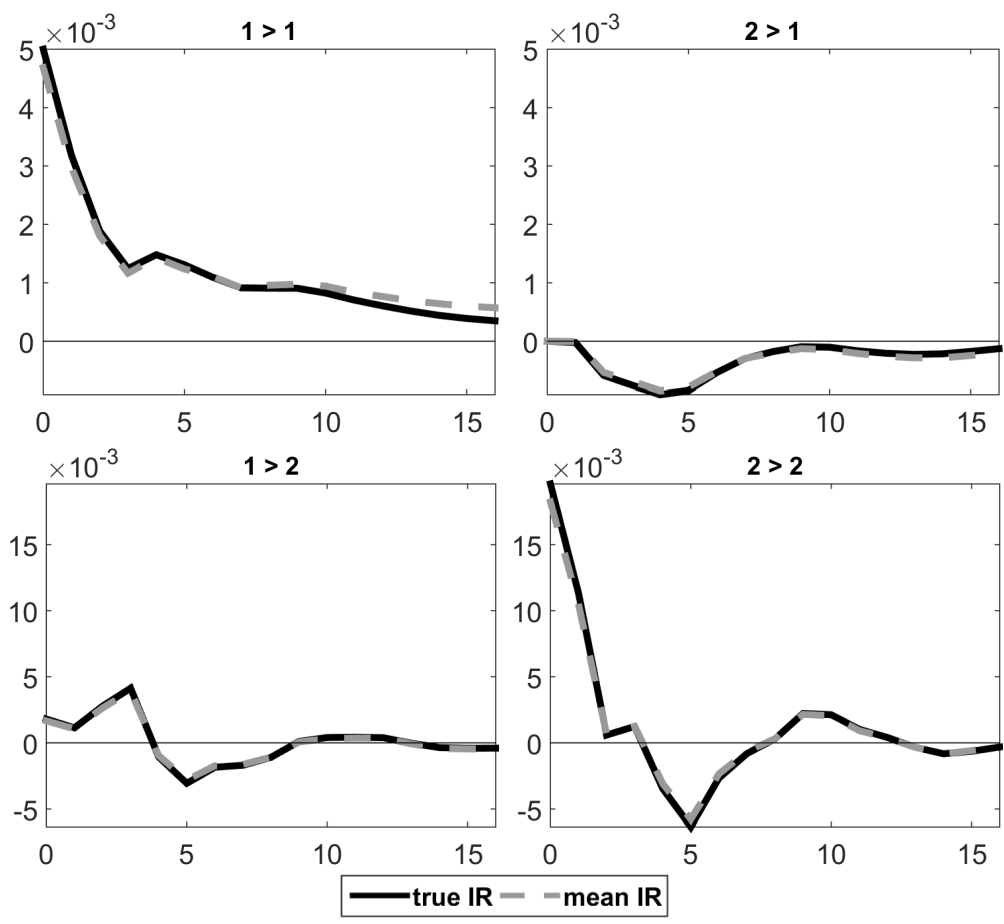

Figure 3.1: True and mean estimated impulse response functions of the $\operatorname{VAR}(4)$. The response of variable $j$ to a shock in variable $i$ is labeled $i>j . T=80$.

is being selected by the BIC at $T=160$, and the average sBIC weight is also $33 \%$, while for AIC the corresponding values of $84 \%$ and $72 \%$ are further apart. This means that the sBIC estimator delivers weights that are more concentrated on one model than the sAIC estimator weights, and also hints at the asymptotic properties: While asymptotically the sBIC estimator concentrates all weight on one model, the sAIC weights can still be dispersed among many.

Figures 3.2 and 3.3 show the MSE performance of the AIC-based methods (AIC and sAIC) compared to the true lag order method. While for $T=80$ for horizons around $h=5$, for example, the true lag method performs notably better than the other estimators, for $T=160$ such large differences have mostly disappeared. At $T=160$ the MSE of the selection and averaging methods are very similar. Judging by Table 3.1 we do not expect these observations to change when increasing the sample sizes beyond $T=160$. The averaging method's MSE is lower than that of the selection method, a fact that we also observe for another simulation setting below, cf. Figure 3.13, and that is in agreement with findings in other papers named in the introduction.

Interestingly, for large horizons the impulse response $1 \rightarrow 1$ in Figure 3.2 is more accurately (in the MSE sense) estimated by the AIC-based methods than 


\begin{tabular}{cl|ccccccccc} 
& $T \backslash p$ & 1 & 2 & 3 & 4 & 5 & 6 & 7 & 8 & 9 \\
\hline AIC & 80 & 11 & 8 & 1 & 62 & 9 & 4 & 2 & 1 & $\cdot$ \\
& 160 & 0 & 1 & 0 & 84 & 9 & 3 & 1 & 1 & 0 \\
& 200 & 0 & 0 & 0 & 85 & 9 & 3 & 1 & 1 & 0 \\
& 300 & 0 & 0 & 0 & 86 & 9 & 3 & 1 & 1 & 0 \\
& 400 & 0 & 0 & 0 & 87 & 9 & 2 & 1 & 0 & 0 \\
& 500 & 0 & 0 & 0 & 88 & 8 & 2 & 1 & 0 & 0 \\
& 5000 & 0 & 0 & 0 & 89 & 8 & 2 & 1 & 0 & 0 \\
\hline sAIC & 80 & 11 & 10 & 3 & 52 & 13 & 6 & 3 & 2 & $\cdot$ \\
& 160 & 0 & 1 & 0 & 72 & 16 & 5 & 2 & 1 & 1 \\
& 200 & 0 & 0 & 0 & 74 & 16 & 5 & 2 & 1 & 0 \\
& 300 & 0 & 0 & 0 & 76 & 16 & 5 & 2 & 1 & 0 \\
& 5000 & 0 & 0 & 0 & 76 & 16 & 5 & 2 & 1 & 0
\end{tabular}

Table 3.1: AIC selection frequencies alongside sAIC average model weights for different sample sizes, both as percentages.

\begin{tabular}{cl|cccccccc} 
& $T \backslash p$ & 1 & 2 & 3 & 4 & 5 & 6 & 7 & 8 \\
\hline BIC & 80 & 87 & 8 & 0 & 5 & 0 & 0 & 0 & 0 \\
& 160 & 57 & 10 & 0 & 33 & 0 & 0 & 0 & 0 \\
& 200 & 36 & 8 & 0 & 56 & 0 & 0 & 0 & 0 \\
& 300 & 5 & 2 & 0 & 93 & 0 & 0 & 0 & 0 \\
& 400 & 0 & 0 & 0 & 99 & 0 & 0 & 0 & 0 \\
& 500 & 0 & 0 & 0 & 100 & 0 & 0 & 0 & 0 \\
\hline sBIC & 80 & 83 & 11 & 0 & 6 & 0 & 0 & 0 & 0 \\
& 160 & 55 & 12 & 0 & 33 & 0 & 0 & 0 & 0 \\
& 200 & 35 & 9 & 0 & 55 & 0 & 0 & 0 & 0 \\
& 300 & 6 & 3 & 0 & 92 & 0 & 0 & 0 & 0 \\
& 400 & 0 & 0 & 0 & 99 & 0 & 0 & 0 & 0 \\
& 500 & 0 & 0 & 0 & 100 & 0 & 0 & 0 & 0 \\
\hline
\end{tabular}

Table 3.2: BIC selection frequencies alongside sBIC average model weights for different sample sizes, both as percentages.

by the true lag method. Figure 3.4 shows the variances of the individual models which explain this finding. The sAIC method assigns positive weights to smaller models with a much lower variance. We refer to Section 3.A.6 for details on the MSE decomposition. There we show that the estimator's variance explains most of the MSE performance for the sAIC-based estimator, while for the sBIC-based estimator the squared bias component also contributes significantly to the MSE.

Even though the BIC-based methods are consistent, the AIC-based methods perform better in our simulation study. This is the case because the overselection of the AIC-based methods is less of a problem than the underselection by the BIC-based methods. Small models induce a bias while large models do not. 


\section{CHAPTER 3}
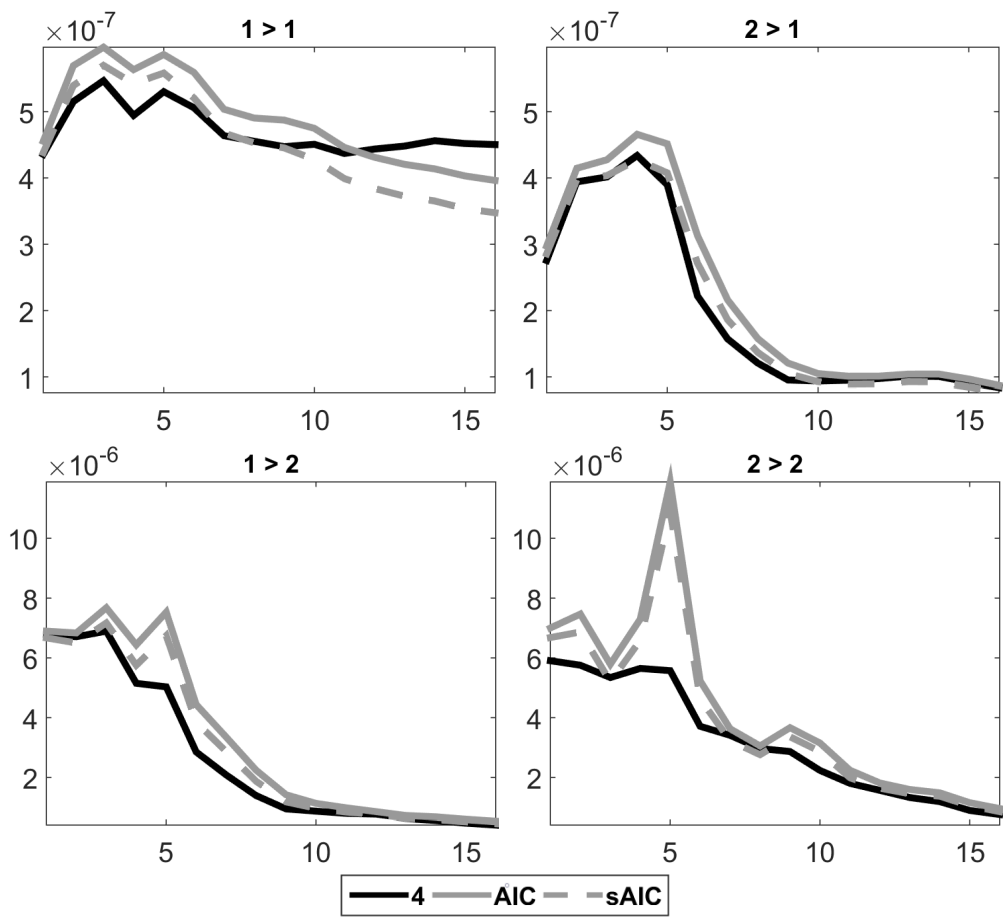

Figure 3.2: MSE of impulse response coefficient estimators. The response of variable $j$ to a shock in variable $i$ is labeled $i>j$. The averaging methods outperform the selection methods. $T=80$.

The increased variance of the large models' estimators seems to be less of a problem. The comparably bad performance of the BIC-based selection method for constructing confidence intervals is already documented in Kilian (2001): The effective coverage of the BIC-based selection method lies below $10 \%$ for some impulse response coefficients, whereas the effective coverage of the AIC estimator is always above $70 \%$. For our averaging setting we obtain similar results. Figure 3.5 documents the comparably worse performance of the BIC-based methods in terms of MSE. The maximal MSE of the BIC-based method for impulse response $2 \rightarrow 1$ is $4 \cdot 10^{-7}$ while it is at around $2 \cdot 10^{-7}$ for the AIC-based methods, cf. Figure 3.3. Also the difference to the MSE of the true lag order method is still comparably large at $T=160$. Based on the results of our simulations we recommend to rely on the AIC-based methods instead of using the BIC-based methods. In this point we agree with Lütkepohl et al. (2015), who also shortly discuss their decision to apply AIC rather than other model selection criteria in their study. 

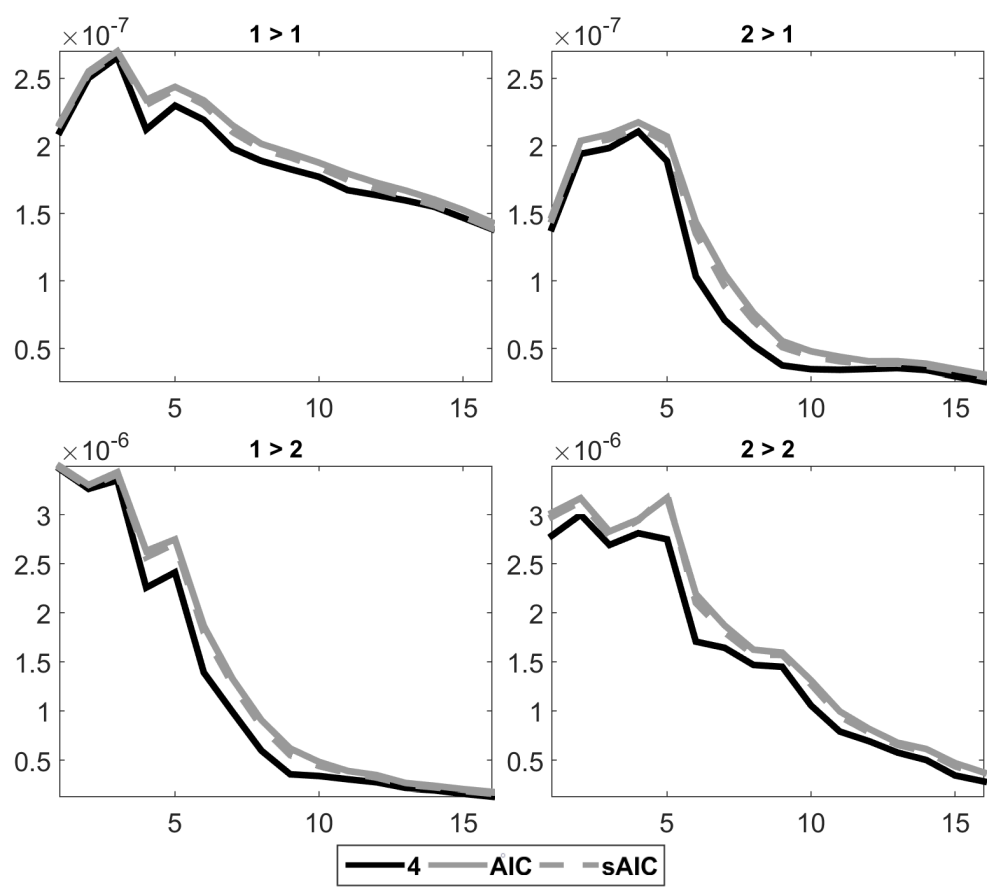

Figure 3.3: MSE of IR coefficient estimators. The response of variable $j$ to a shock in variable $i$ is labeled $i>j . T=160$.

\section{Coverage}

Figures 3.6 and 3.7 show the effective coverages of the pointwise confidence intervals for a nominal coverage of $95 \%$. Here the averaging method does not reach a coverage closer to the nominal rate than the selection method does in general. Instead we observe that often the sAIC-based effective coverage is lower than that of the AIC-based method.

Based on the pointwise confidence intervals we calculate joint confidence bands using a method termed adjusted Bonferroni by Lütkepohl et al. (2015). They discuss different methods of constructing joint bands based on asymptotics as well as bootstrap methods. Bonferroni joint confidence bands are obtained by joining pointwise bands with a nominal coverage of $1-\alpha / H$ if the impact effect is restricted to zero, and $1-\alpha /(H+1)$ if it is not restricted to zero for this particular impulse response function. Here $H$ is the number of horizons considered. By dividing the significance level by $H$, Bonferroni bands become conservative by construction. So Lütkepohl et al. (2015) suggest to use the adjusted Bonferroni method which they find to perform well compared to all other tested methods when the underlying time series is stationary. This method is aimed at minimizing the width of the confidence band while keeping a good coverage, and we will use it here.

Table 3.3 shows the effective coverages of the adjusted Bonferroni bands. It 

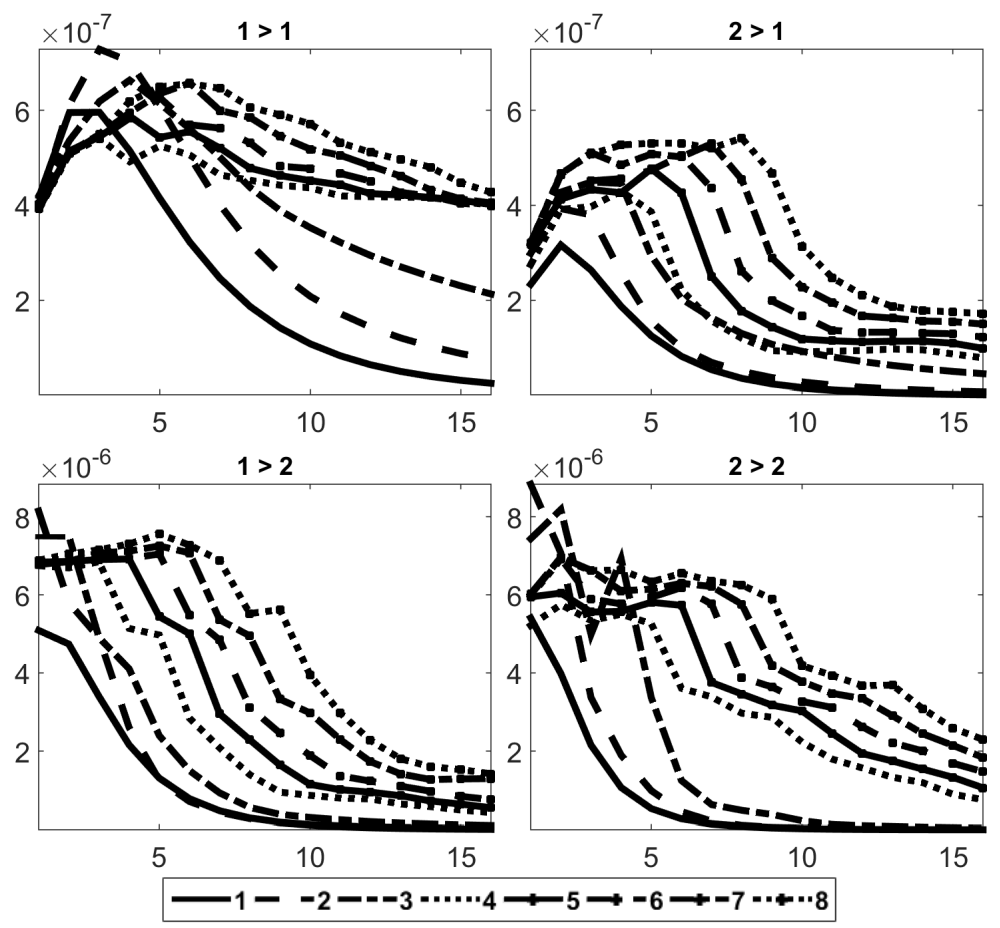

Figure 3.4: Empirical variances of the $\operatorname{VAR}(i)$ based impulse response estimates, $i=1, \ldots, 8$. The response of variable $j$ to a shock in variable $i$ is labeled $i>j . \mathrm{T}=80$.

is instructive to inspect Table 3.3 together with Figures 3.6, 3.7. For $T=80$ the coverages of the pointwise AIC-based intervals are below those of the true lag method. The same holds for the coverage of the confidence bands. For IR $1 \rightarrow 1$ for example it is 87.3 for the true lag method, and 81.6 for the AIC-based method. For sample size $T=160$ we make the opposite observation: The AIC-based method tends to have higher coverage than the true lag method. Also analogously to the pointwise effective coverages the sAIC-based coverages of the adjusted Bonferroni method tend to be lower than the corresponding AIC-based coverages. These two observations still hold for the larger sample size of $T=500$, such that analogously to the evidence from Table 3.1 above, we suspect that these observations will still hold for even larger sample sizes.

Except for the impulse responses $2 \rightarrow 1$ at $T=160$ and $T=500$ the effective coverage of the AIC-based confidence band is closer to the nominal $95 \%$ than that of the sAIC-based one. This is mild evidence that a researcher having to decide between AIC-based and sAIC-based bands should apply the former. Compared to the true lag method for a sample size of $T=160$ the AIC-based methods have better effective coverage even than the true lag method.

What Kilian (1998a) pointed out for the selection based estimator we also find 

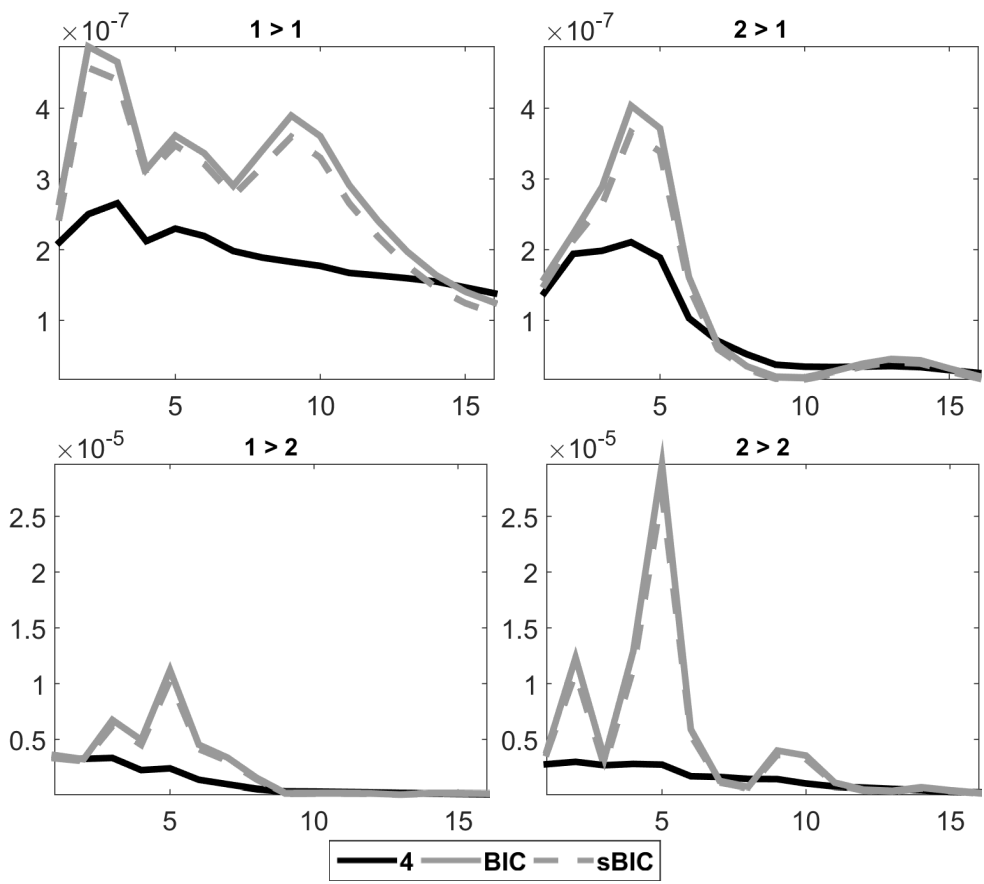

Figure 3.5: MSE of BIC-based impulse response coefficient estimators.

The response of variable $j$ to a shock in variable $i$ is labeled $i>j . T=160$.

to hold true for the averaging based estimator: The exogenous weights bootstrap method has worse coverage than the endogenous bootstrap. For $T=80$ the effective coverages of the adjusted Bonferroni method are below $85 \%$ for all four impulse response coefficients (results not shown).

Figure 3.8 shows the extraordinarily low coverage that results from choosing a lag order that often is too low for generating the bootstrapped data. For this graph the BIC-based methods were applied. This means that in two different steps of the algorithm small models are used too often even for $T=160$, in the bootstrap sample generation step and when estimating the impulse response coefficients, cf. our bootstrap algorithm above. This leads to the coverages of even the true lag order method to decline to below $40 \%$ for impulse response $2 \rightarrow 2$ for example. Hence, with this evidence similar to Kilian (2001) we recommend to not use BIC or sBIC in our setting, but use the AIC based estimators instead.

In summary our simulation evidence based on the setup of Kilian (2001) shows that the theoretically derived rates of decline of the weights of Lemma 3.1 can be observed for realistic sample sizes of relevant data already. As these rates correspond to the selection frequencies of the corresponding model selection estimators (AIC and BIC) it is not surprising to see that the performance of the smoothed averaging estimators is similar to the selection estimators in terms of MSE and effective coverage of the confidence intervals. While the MSE of the averaging 


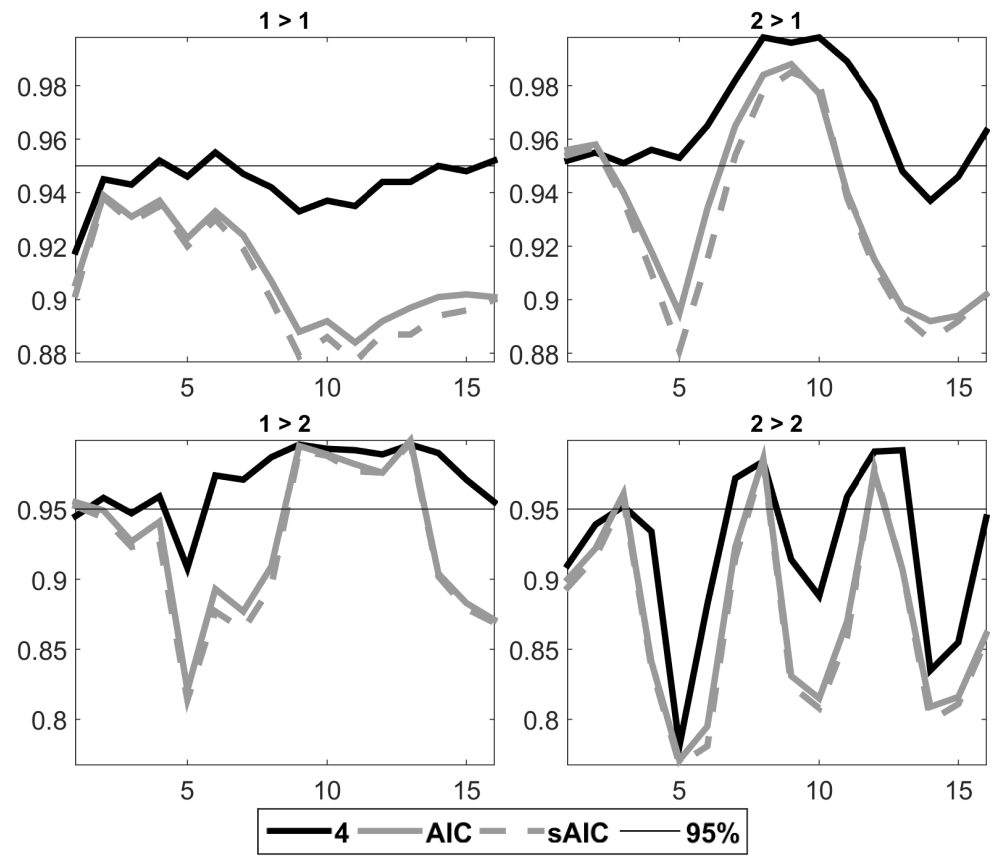

Figure 3.6: Effective coverage (in percent) of the endogenous bootstrapped impulse response coefficients. The response of variable $j$ to a shock in variable $i$ is labeled $i>j$. AIC is used for selecting the lag order of the bootstrap process. The bias correction of Kilian (1998b) is applied to the OLS estimates of $\boldsymbol{\beta} . T=80$.

estimators is found to be lower than that of the selection estimators, the ranking of the estimators largely reverses for the effective coverages. These observations seem to be robust across the simulation settings considered in this chapter, while the reasons for these opposing behaviours are not obvious and remain a topic for future research.

\subsection{Conclusion}

This chapter extends the theoretical basis for using averaging methods, and demonstrates their usefulness. We show that certain properties of model selection estimators carry over to their model averaging counterparts based on smoothed weights. The sBIC-based estimator is still consistent and the sAIC-based estimator puts no weight on small models asymptotically. Our setting is that of impulse response coefficients of multivariate stationary time series. But our results, which build on Zhang (2015), hold analogously for other smooth functions of OLS estimators in VARs. Our second contribution is to show asymptotic validity for a bootstrap method to obtain confidence invervals for the averaged estimators. 

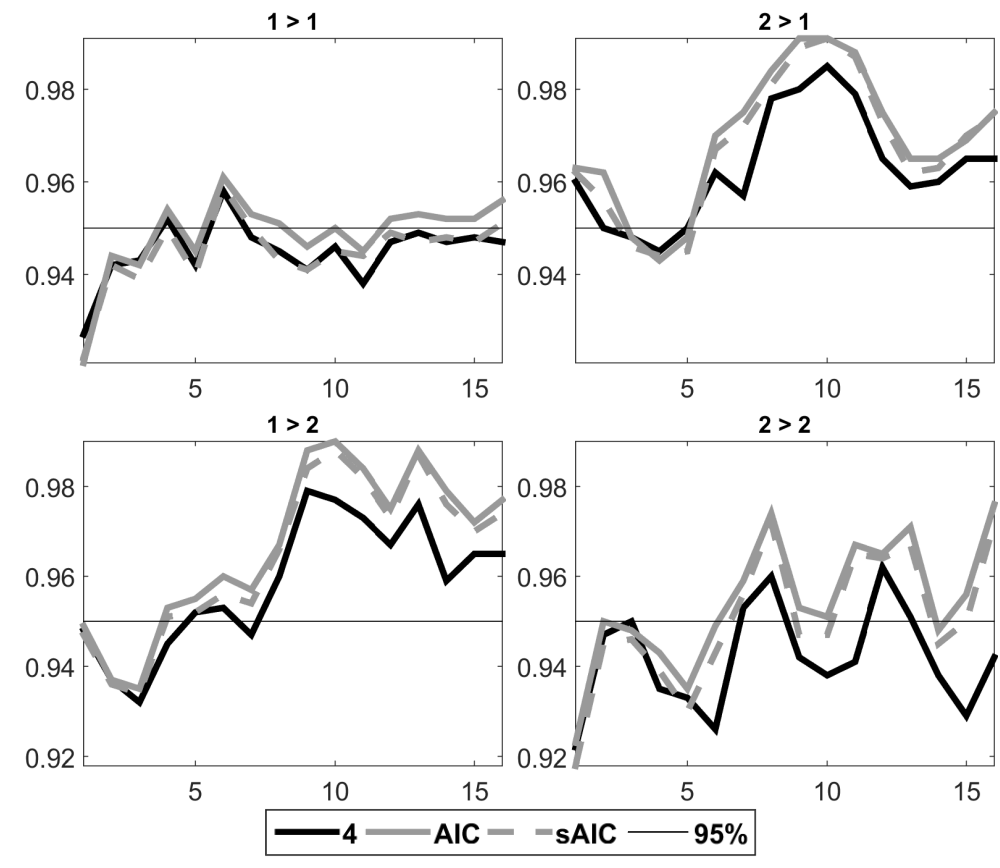

Figure 3.7: Effective coverage (in percent) of the endogenous bootstrapped impulse response coefficients. The response of variable $j$ to a shock in variable $i$ is labeled $i>j$. AIC is used for selecting the lag order of the bootstrap process. The bias correction of Kilian (1998b) is applied to the OLS estimates of $\boldsymbol{\beta} . T=160$.

In empirically relevant VAR simulation settings we observe that model averaging is superior to model selection for point estimation of impulse response coefficients in terms of mean squared error (MSE). A suggestion in this direction was already made by Hansen (2007). We also find that for relatively small sample sizes the sAIC-based method already behaves similarly to the selection method. For the practicioner interested in point estimates this means that by using sAIC instead of AIC she gains accuracy, while at the same time she may still rely on her intuition for the behavior of AIC, because both methods become closer to each other with increasing sample size.

In line with Kilian (2001) our simulations show that even though the BIC-based selection and averaging methods are consistent, they are not useful for sample sizes typical for macroeconomics. In our simulations even for a sample size of $T=160$ the effective coverages of the sBIC-based estimator are remarkably low. Because of the quicker convergence rate of the sAIC weights given in Lemma 3.1 for small models on the other hand, the sAIC-based method has good risk and coverages for sample sizes from $T=160$ already. We also find that the effective coverage of the AIC-based joint confidence bands is better than that of the true lag method. 


\section{CHAPTER 3}

\begin{tabular}{ll|cccc} 
method & $T \backslash \mathrm{IR}$ & $1 \rightarrow 1$ & $2 \rightarrow 1$ & $1 \rightarrow 2$ & $2 \rightarrow 2$ \\
\hline \multirow{2}{*}{ true lag } & 80 & 87.3 & 95.2 & 95.8 & 75.7 \\
& 160 & 90.2 & 93.9 & 91.3 & 86.9 \\
& 500 & 91.3 & 93.4 & 91.1 & 88.9 \\
\hline AIC & 80 & 81.6 & 90.5 & 85.6 & 71.6 \\
& 160 & 90.5 & 95.8 & 94.8 & 89.9 \\
& 500 & 92.9 & 96.7 & 95.6 & 92.3 \\
\hline sAIC & 80 & 80.1 & 89.0 & 84.6 & 70.0 \\
& 160 & 89.7 & 95.0 & 93.5 & 88.9 \\
& 500 & 92.5 & 96.1 & 93.8 & 91.1 \\
\hline
\end{tabular}

Table 3.3: Effective coverage (in percent) of the nominal $95 \%$ adjusted Bonferroni joint confidence bands.

In Chapter 4 we will revisit some empirical studies and apply model averaging methods of several model criteria to assess the applicability of averaging methods more in detail.

\section{A Appendix}

For $p<p_{0}$ we introduce the following notation. We partition $\boldsymbol{B}$ and $\boldsymbol{Z}$, matrices corresponding to the true DGP, into submatrices:

$$
\begin{aligned}
\boldsymbol{B} & =\left(\boldsymbol{B}_{1}, \boldsymbol{B}_{2}\right), \text { with } \\
\boldsymbol{B}_{1} & =\left(\boldsymbol{A}_{1}, \ldots, \boldsymbol{A}_{p}\right), \\
\boldsymbol{B}_{2} & =\left(\boldsymbol{A}_{p+1}, \ldots, \boldsymbol{A}_{p_{0}}\right), \text { and } \\
\boldsymbol{Z} & =\left(\begin{array}{l}
\boldsymbol{Z}_{1} \\
\boldsymbol{Z}_{2}
\end{array}\right), \text { with }
\end{aligned}
$$

With this notation adopted from Paulsen (1984), cf. the proof of Theorem 1, and Anderson (2003) one has to keep in mind that the dimensions of the defined objects depend on $p$. The OLS estimator of the model with the true lag order known is $\widehat{\boldsymbol{B}}=\boldsymbol{Y} \boldsymbol{Z}^{\prime}\left(\boldsymbol{Z} \boldsymbol{Z}^{\prime}\right)^{-1}$, and for the model with $p$ lags we define $\widehat{\boldsymbol{B}}_{p}=\boldsymbol{Y} \boldsymbol{Z}_{1}^{\prime}\left(\boldsymbol{Z}_{1} \boldsymbol{Z}_{1}^{\prime}\right)^{-1}$. We define $\widehat{\boldsymbol{B}}_{1}$, and $\widehat{\boldsymbol{B}}_{2}$ analogously to $\boldsymbol{B}_{1}, \boldsymbol{B}_{2}$ above, so that $\widehat{\boldsymbol{B}}=\left(\widehat{\boldsymbol{B}}_{1}, \widehat{\boldsymbol{B}}_{2}\right)$. Note that in general $\widehat{\boldsymbol{B}}_{1} \neq \widehat{\boldsymbol{B}}_{p}$.

\section{A.1 Proof of Lemma 3.1}

We derive the rates of convergence to 0 for the weights of model $p$ for the two different cases $p<p_{0}$, and $p_{0}<p$.

Consider the case $p<p_{0}$. We can write

$$
\boldsymbol{Z} \boldsymbol{Z}^{\prime}=\left(\begin{array}{ll}
\boldsymbol{Z}_{1} \boldsymbol{Z}_{1}^{\prime} & \boldsymbol{Z}_{1} \boldsymbol{Z}_{2}^{\prime} \\
\boldsymbol{Z}_{2} \boldsymbol{Z}_{1}^{\prime} & \boldsymbol{Z}_{2} \boldsymbol{Z}_{2}^{\prime}
\end{array}\right)
$$



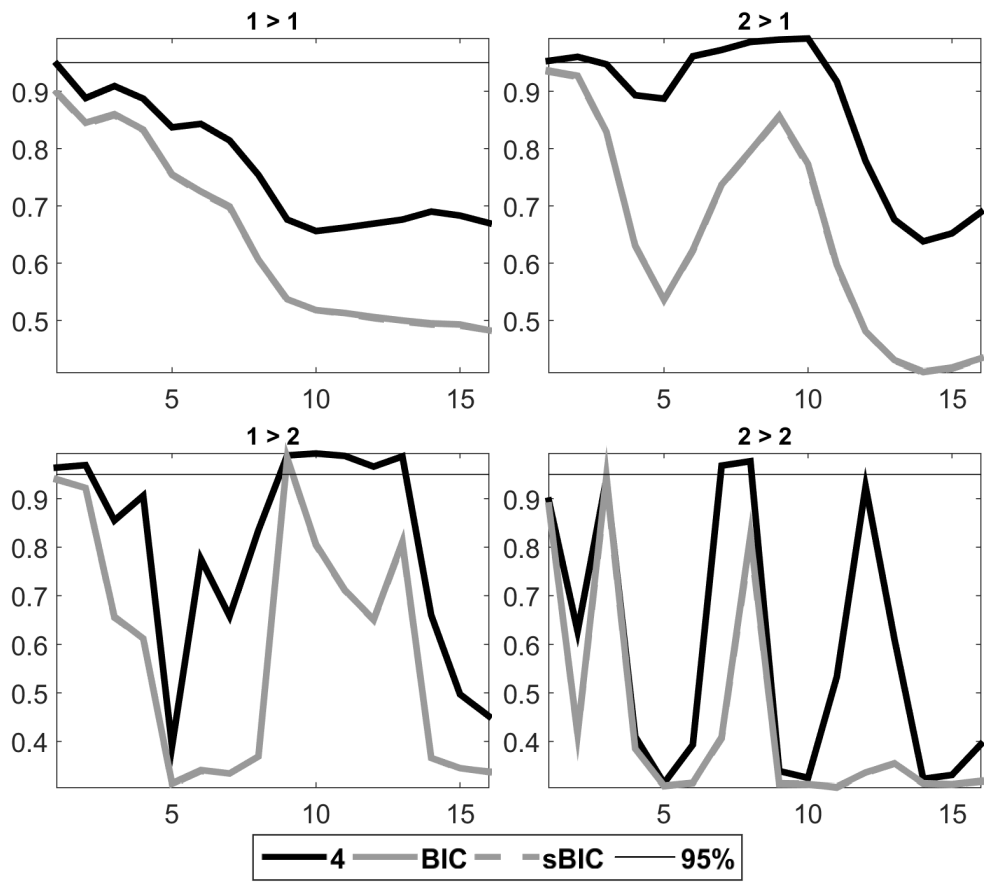

Figure 3.8: Effective coverage (in percent) of the endogenous bootstrapped impulse response coefficients. The response of variable $j$ to a shock in variable $i$ is labeled $i>j$. BIC is used for selecting the lag order of the bootstrap process. The bias correction is applied to the OLS estimates. $T=160$.

Then by rearranging the formula for the OLS estimator along with applying the definitions above we get

$$
\begin{aligned}
& \left(\widehat{B}_{1} \boldsymbol{Z}_{1} \boldsymbol{Z}_{1}^{\prime}+\widehat{B}_{2} \boldsymbol{Z}_{2} \boldsymbol{Z}_{1}^{\prime}, \widehat{B}_{1} \boldsymbol{Z}_{1} \boldsymbol{Z}_{2}^{\prime}+\widehat{B}_{2} \boldsymbol{Z}_{2} \boldsymbol{Z}_{2}^{\prime}\right)=\widehat{\boldsymbol{B}} \boldsymbol{Z} \boldsymbol{Z}^{\prime}=\boldsymbol{Y} \boldsymbol{Z}^{\prime}=\left(\boldsymbol{Y} \boldsymbol{Z}_{1}^{\prime}, \boldsymbol{Y} \boldsymbol{Z}_{2}^{\prime}\right) \\
& \Rightarrow \widehat{\boldsymbol{B}}_{p}-\widehat{\boldsymbol{B}}_{1}=\boldsymbol{Y} \boldsymbol{Z}_{1}^{\prime}\left(\boldsymbol{Z}_{1} \boldsymbol{Z}_{1}^{\prime}\right)^{-1}-\boldsymbol{Y} \boldsymbol{Z}_{1}^{\prime}\left(\boldsymbol{Z}_{1} \boldsymbol{Z}_{1}^{\prime}\right)^{-1}+\widehat{\boldsymbol{B}}_{2} \boldsymbol{Z}_{2} \boldsymbol{Z}_{1}^{\prime}\left(\boldsymbol{Z}_{1} \boldsymbol{Z}_{1}^{\prime}\right)^{-1} \\
& =\widehat{B}_{2} \boldsymbol{Z}_{2} \boldsymbol{Z}_{1}^{\prime}\left(\boldsymbol{Z}_{1} \boldsymbol{Z}_{1}^{\prime}\right)^{-1} \text {. }
\end{aligned}
$$

We can use this to write

$$
\begin{aligned}
\boldsymbol{Y}-\boldsymbol{B} \boldsymbol{Z} & =\boldsymbol{Y}-\boldsymbol{B}_{1} \boldsymbol{Z}_{1}-\boldsymbol{B}_{2} \boldsymbol{Z}_{2} \underbrace{-\widehat{\boldsymbol{B}} \boldsymbol{Z}+\widehat{\boldsymbol{B}}_{1} \boldsymbol{Z}_{1}+\widehat{\boldsymbol{B}}_{2} \boldsymbol{Z}_{2}}_{=0} \\
& =(\boldsymbol{Y}-\widehat{\boldsymbol{B}} \boldsymbol{Z})+\left(\widehat{\boldsymbol{B}}_{2}-\boldsymbol{B}_{2}\right) \boldsymbol{Z}_{2}+\left(\widehat{\boldsymbol{B}}_{p}-\boldsymbol{B}_{1}\right) \boldsymbol{Z}_{1}-\left(\widehat{\boldsymbol{B}}_{p}-\widehat{\boldsymbol{B}}_{1}\right) \boldsymbol{Z}_{1} \\
& =(\boldsymbol{Y}-\widehat{\boldsymbol{B}} \boldsymbol{Z})+\left(\widehat{\boldsymbol{B}}_{2}-\boldsymbol{B}_{2}\right) \boldsymbol{Z}_{2}+\left(\widehat{\boldsymbol{B}}_{p}-\boldsymbol{B}_{1}\right) \boldsymbol{Z}_{1}-\widehat{\boldsymbol{B}}_{2} \boldsymbol{Z}_{2} \boldsymbol{Z}_{1}^{\prime}\left(\boldsymbol{Z}_{1} \boldsymbol{Z}_{1}^{\prime}\right)^{-1} \boldsymbol{Z}_{1} \\
& =(\boldsymbol{Y}-\widehat{\boldsymbol{B}} \boldsymbol{Z})+\left(\widehat{\boldsymbol{B}}_{p}-\boldsymbol{B}_{1}\right) \boldsymbol{Z}_{1}+\widehat{\boldsymbol{B}}_{2}\left(\boldsymbol{Z}_{2}-\boldsymbol{Z}_{2} \boldsymbol{Z}_{1}^{\prime}\left(\boldsymbol{Z}_{1} \boldsymbol{Z}_{1}^{\prime}\right)^{-1} \boldsymbol{Z}_{1}\right)-\boldsymbol{B}_{2} \boldsymbol{Z}_{2},
\end{aligned}
$$

where the second equality is obtained by simply adding and subtracting a term, so adding 0 . Subtracting $\left(\widehat{\boldsymbol{B}}_{p}-\boldsymbol{B}_{1}\right) \boldsymbol{Z}_{1}-\boldsymbol{B}_{2} \boldsymbol{Z}_{2}$ from both sides (second and last 


\section{CHAPTER 3}

line) of Eqn (3.11) we get

$$
\begin{aligned}
(\boldsymbol{Y}-\widehat{\boldsymbol{B}} \boldsymbol{Z})+\widehat{\boldsymbol{B}}_{2} \boldsymbol{Z}_{2}-\left(\widehat{\boldsymbol{B}}_{p}-\widehat{\boldsymbol{B}}_{1}\right) \boldsymbol{Z}_{1} & =\boldsymbol{Y}-\widehat{\boldsymbol{B}}_{p} \boldsymbol{Z}_{1} \\
& =(\boldsymbol{Y}-\widehat{\boldsymbol{B}} \boldsymbol{Z})+\widehat{\boldsymbol{B}}_{2}\left(\boldsymbol{Z}_{2}-\boldsymbol{Z}_{2} \boldsymbol{Z}_{1}^{\prime}\left(\boldsymbol{Z}_{1} \boldsymbol{Z}_{1}^{\prime}\right)^{-1} \boldsymbol{Z}_{1}\right) .
\end{aligned}
$$

$(\boldsymbol{Y}-\widehat{\boldsymbol{B}} \boldsymbol{Z})$ is orthogonal to $\widehat{\boldsymbol{B}}_{2}\left(\boldsymbol{Z}_{2}-\boldsymbol{Z}_{2} \boldsymbol{Z}_{1}^{\prime}\left(\boldsymbol{Z}_{1} \boldsymbol{Z}_{1}^{\prime}\right)^{-1} \boldsymbol{Z}_{1}\right)$ : Since $\widehat{\boldsymbol{B}} \boldsymbol{Z}=$

$\boldsymbol{Y} \boldsymbol{Z}^{\prime}\left(\boldsymbol{Z} \boldsymbol{Z}^{\prime}\right)^{-1} \boldsymbol{Z}$ is the projection of $\boldsymbol{Y}$ into the $\boldsymbol{Z}$-space, $\boldsymbol{Y}-\widehat{\boldsymbol{B}} \boldsymbol{Z}$ is orthogonal to the $\boldsymbol{Z}$-space. But the second summand is an object of the $\boldsymbol{Z}$-space. Because of this orthogonality the cross-terms in the following equation are zero and the estimated innovation covariance matrix that we obtain by using the small model with $p$ lags can now be written as

$$
\begin{aligned}
T \widehat{\boldsymbol{\Sigma}}_{p}= & \left(\boldsymbol{Y}-\widehat{\boldsymbol{B}}_{p} \boldsymbol{Z}_{1}\right)\left(\boldsymbol{Y}-\widehat{\boldsymbol{B}}_{p} \boldsymbol{Z}_{1}\right)^{\prime} \\
= & (\boldsymbol{Y}-\widehat{\boldsymbol{B}} \boldsymbol{Z})(\boldsymbol{Y}-\widehat{\boldsymbol{B}} \boldsymbol{Z})^{\prime} \\
& +\widehat{\boldsymbol{B}}_{2}\left(\boldsymbol{Z}_{2}-\boldsymbol{Z}_{2} \boldsymbol{Z}_{1}^{\prime}\left(\boldsymbol{Z}_{1} \boldsymbol{Z}_{1}^{\prime}\right)^{-1} \boldsymbol{Z}_{1}\right)\left(\boldsymbol{Z}_{2}-\boldsymbol{Z}_{2} \boldsymbol{Z}_{1}^{\prime}\left(\boldsymbol{Z}_{1} \boldsymbol{Z}_{1}^{\prime}\right)^{-1} \boldsymbol{Z}_{1}\right)^{\prime} \widehat{\boldsymbol{B}}_{2}^{\prime} \\
= & T \widehat{\boldsymbol{\Sigma}}_{p_{0}}+\widehat{\boldsymbol{B}}_{2}\left(\boldsymbol{Z}_{2} \boldsymbol{Z}_{2}^{\prime}-\boldsymbol{Z}_{2} \boldsymbol{Z}_{1}^{\prime}\left(\boldsymbol{Z}_{1} \boldsymbol{Z}_{1}^{\prime}\right)^{-1} \boldsymbol{Z}_{1} \boldsymbol{Z}_{2}^{\prime}\right) \widehat{\boldsymbol{B}}_{2}^{\prime} .
\end{aligned}
$$

Both summands are positive definite symmetric matrices.

Since $\boldsymbol{\Omega}$ is assumed positive definite (cf. Eqn (3.2)), it follows with

$$
|\boldsymbol{\Omega}|=\left|\left(\begin{array}{ll}
\boldsymbol{\Omega}_{11} & \boldsymbol{\Omega}_{12} \\
\boldsymbol{\Omega}_{21} & \boldsymbol{\Omega}_{22}
\end{array}\right)\right|=\left|\boldsymbol{\Omega}_{11}\right|\left|\boldsymbol{\Omega}_{22}-\boldsymbol{\Omega}_{21} \boldsymbol{\Omega}_{11}^{-1} \boldsymbol{\Omega}_{12}\right|
$$

from Rao (1973), that $\left|\boldsymbol{\Omega}_{22}-\boldsymbol{\Omega}_{21} \boldsymbol{\Omega}_{11}^{-1} \boldsymbol{\Omega}_{12}\right|>0$ for any decomposition of $\boldsymbol{\Omega}$ into submatrices $\boldsymbol{\Omega}_{11}, \boldsymbol{\Omega}_{12}, \boldsymbol{\Omega}_{21}, \boldsymbol{\Omega}_{22}$. Applying the continuous mapping theorem this shows that the second summand of the RHS of Eqn (3.13) converges to a positive real number in probability.

We now look at the ratio of sBIC model weights. Use Eqn (3.6) to find

$$
\begin{aligned}
\frac{\widehat{w}_{B I C, p}}{\widehat{w}_{B I C, p_{0}}} & =\left(\frac{\left|\widehat{\boldsymbol{\Sigma}}_{p_{0}}\right|}{\left|\widehat{\boldsymbol{\Sigma}}_{p}\right|}\right)^{T / 2} T^{\left(p_{0}-p\right) d^{2} / 2} \\
& =\left(\frac{\left|\widehat{\boldsymbol{\Sigma}}_{p_{0}}\right|}{\left|\widehat{\boldsymbol{\Sigma}}_{p_{0}}+T^{-1} \widehat{\boldsymbol{B}}_{2}\left(\boldsymbol{Z}_{2} \boldsymbol{Z}_{2}^{\prime}-\boldsymbol{Z}_{2} \boldsymbol{Z}_{1}^{\prime}\left(\boldsymbol{Z}_{1} \boldsymbol{Z}_{1}^{\prime}\right)^{-1} \boldsymbol{Z}_{1} \boldsymbol{Z}_{2}^{\prime}\right) \widehat{\boldsymbol{B}}_{2}^{\prime}\right|}\right)^{T / 2} T^{\left(p_{0}-p\right) d^{2} / 2} .
\end{aligned}
$$

It is well known that $\left|\widehat{\boldsymbol{\Sigma}}_{p_{0}}\right| /\left|\widehat{\boldsymbol{\Sigma}}_{p}\right| \leq 1$ (cf. Lütkepohl (1990), p. 147), and the probability limit is even strictly smaller than 1 , as just discussed. So $\frac{\widehat{w}_{B I C, p}}{\widehat{w}_{B I C, p_{0}}}=$ $O_{p}\left(c^{T} T^{\left(p_{0}-p\right) d^{2} / 2}\right)$ holds for some $c \in(0,1)$, and since $\widehat{w}_{B I C, p} \in[0,1]$ for all $p \in\{1, \ldots, \bar{p}\}$ also

$$
\begin{aligned}
& \widehat{w}_{B I C, p}=O_{p}\left(c_{1}^{T} T^{\left(p_{0}-p\right) d^{2} / 2}\right), \text { and by completely analogous arguments } \\
& \widehat{w}_{A I C, p}=O_{p}\left(c_{2}^{T} \exp \left(\left(p_{0}-p\right) d^{2} / 2\right)\right)=O_{p}\left(c_{2}^{T}\right)
\end{aligned}
$$

for some $c_{1}, c_{2} \in(0,1)$. 


\section{A. APPENDIX}

Now consider the case $p>p_{0}$. Quinn (1980) shows consistency of BIC: $\operatorname{Prob}\left(\widehat{w}_{B I C, p}>\widehat{w}_{B I C, p_{0}}\right)=\operatorname{Prob}\left(\frac{\widehat{w}_{B I C, p}}{\widehat{w}_{B I C, p_{0}}}>1\right) \rightarrow 0$. Then we can conclude,

$$
\begin{aligned}
\frac{\widehat{w}_{B I C, p}}{\widehat{w}_{B I C, p_{0}}}=\left(\frac{\left|\widehat{\boldsymbol{\Sigma}}_{p_{0}}\right|}{\left|\widehat{\boldsymbol{\Sigma}}_{p}\right|}\right)^{T / 2} T^{\left(p_{0}-p\right) d^{2} / 2}=O_{p}\left(T^{\left(p_{0}-p\right) d^{2} / 2}\right) \\
\Rightarrow \widehat{w}_{B I C, p}=O_{p}\left(T^{\left(p_{0}-p\right) d^{2} / 2}\right),
\end{aligned}
$$

where the last claim follows with $\widehat{w}_{B I C, p_{0}} \in[0,1]$. Note that no faster rate of convergence of $\widehat{w}_{p}$ can be established, because $\left|\widehat{\boldsymbol{\Sigma}}_{p_{0}}\right| /\left|\widehat{\boldsymbol{\Sigma}}_{p}\right| \geq 1$.

\section{A.2 Proof of Theorem 3.2}

The distribution of the averaged estimator is given by

$$
\begin{aligned}
\sqrt{T}\left(\widehat{\theta}\left(\widehat{\boldsymbol{w}}_{x I C}\right)-\theta\right)= & \sqrt{T}\left(\widehat{w}_{x I C, 1} \widehat{\theta}_{1}+\widehat{w}_{x I C, 2} \widehat{\theta}_{2}+\ldots+\widehat{w}_{x I C, \bar{p}} \widehat{\theta}_{\bar{p}}-\theta\right) \\
= & \sum_{p=1}^{p_{0}-1} \sqrt{T} \widehat{w}_{x I C, p} \widehat{\theta}_{p}+\sum_{p=p_{0}+2}^{\bar{p}} \sqrt{T} \widehat{w}_{x I C, p} \widehat{\theta}_{p} \\
& +\widehat{w}_{x I C, p_{0}} \sqrt{T}\left(\widehat{\theta}_{p_{0}}-\theta\right)+\widehat{w}_{x I C, p_{0}+1} \sqrt{T}\left(\widehat{\theta}_{p_{0}+1}-\theta\right) .
\end{aligned}
$$

To show $\widehat{\theta}_{p}=O_{p}(1)$ for $p<p_{0}$ we note that $\boldsymbol{Z}_{1} \boldsymbol{Z}_{1}^{\prime}$, and $\boldsymbol{Z} \boldsymbol{Z}_{1}^{\prime}$ are submatrices of $\boldsymbol{Z} \boldsymbol{Z}^{\prime}$, and write

$$
\begin{aligned}
\widehat{\boldsymbol{B}}_{p} & =\boldsymbol{Y} \boldsymbol{Z}_{1}^{\prime}\left(\boldsymbol{Z}_{1} \boldsymbol{Z}_{1}^{\prime}\right)^{-1}=\boldsymbol{B} \boldsymbol{Z} \boldsymbol{Z}_{1}^{\prime}\left(\boldsymbol{Z}_{1} \boldsymbol{Z}_{1}^{\prime}\right)^{-1}+\boldsymbol{U} \boldsymbol{Z}_{1}^{\prime}\left(\boldsymbol{Z}_{1} \boldsymbol{Z}_{1}^{\prime}\right)^{-1} \\
& =\boldsymbol{B} \frac{1}{T} \boldsymbol{Z} \boldsymbol{Z}_{1}^{\prime}\left(\frac{1}{T} \boldsymbol{Z}_{1}^{\prime} \boldsymbol{Z}_{1}\right)^{-1}+\frac{1}{\sqrt{T}} \frac{1}{\sqrt{T}} \boldsymbol{U} \boldsymbol{Z}_{1}^{\prime}\left(\frac{1}{T} \boldsymbol{Z}_{1}^{\prime} \boldsymbol{Z}_{1}\right)^{-1} .
\end{aligned}
$$

Then the claim follows from the continuous mapping theorem and Equation (3.2).

Note that from Lemma 3.1 we have for the sBIC weights:

$$
\begin{aligned}
& \widehat{w}_{B I C, p}=O_{p}\left(T^{-1 / 2}\right) \text { for } p=p_{0}+1, \\
& \widehat{w}_{B I C, p}=O_{p}\left(T^{-2 / 2}\right) \text { for } p=p_{0}+2, \text { etc. }
\end{aligned}
$$

So we know

$$
\begin{aligned}
\sum_{p=1}^{p_{0}-1} \sqrt{T} \widehat{w}_{B I C, p} \widehat{\theta}_{p} & =\sum_{p=1}^{p_{0}-1} \sqrt{T} O_{p}\left(c^{T} T^{\left(p_{0}-p\right) / 2}\right) O_{p}(1)=\sqrt{T} O_{p}\left(c^{T} T^{\left(p_{0}-1\right) / 2}\right) \\
& =O_{p}\left(c^{T} T^{p_{0} / 2}\right), \\
\sum_{p=p_{0}+2}^{\bar{p}} \sqrt{T} \widehat{w}_{B I C, p} \widehat{\theta}_{p} & =\sum_{p=p_{0}+2}^{\bar{p}} \sqrt{T} O_{p}\left(T^{\left(p_{0}-p\right) / 2}\right) O_{p}(1)=\sqrt{T} O_{p}\left(T^{-2 / 2}\right)=O_{p}\left(T^{-1 / 2}\right),
\end{aligned}
$$

where $c \in(0,1)$. By Slutsky's Theorem we also know that for the last term of Equation (3.14),

$$
\underbrace{\widehat{w}_{B I C, p_{0}+1} \sqrt{T}}_{O_{p}(1)} \underbrace{\left(\widehat{\theta}_{p_{0}+1}-\theta\right)}_{O_{p}\left(T^{-1 / 2}\right)}=O_{p}\left(T^{-1 / 2}\right) .
$$




\section{CHAPTER 3}

So together the claim follows:

$$
\sqrt{T}\left(\widehat{\theta}\left(\widehat{\boldsymbol{w}}_{B I C}\right)-\theta\right)=\sqrt{T}\left(\widehat{\theta}_{p_{0}}-\theta\right)+O_{p}\left(T^{-1 / 2}\right) .
$$

The sAIC weights for the large models $p>p_{0}$ do not tend to 0 , but are bounded at 1 . Since the estimates $\widehat{\theta}_{p}$ for $p>p_{0}$ are unbiased, analogously to the above argumentation we only obtain

$$
\sqrt{T}\left(\widehat{\theta}\left(\widehat{\boldsymbol{w}}_{A I C}\right)-\theta\right)=O_{p}(1) .
$$

\section{A.3 Derivation of a Bound for $\kappa$}

The expression in the proof of Lemma 3.1, Section 3.A.1, for the case $p>p_{0}$, where $p_{0}$ is the true lag order, becomes

$$
\frac{\widehat{w}_{B I C, p}}{\widehat{w}_{B I C, p_{0}}}=\left(\frac{\left|\widehat{\boldsymbol{\Sigma}}_{p_{0}}\right|}{\left|\widehat{\boldsymbol{\Sigma}}_{p}\right|}\right)^{T / 2 \kappa} T^{\frac{\left(p_{0}-p\right) d^{2}}{2 \kappa}},
$$

when we include the parameter $\kappa$ in the definition of the weights. In order for the last term to be $O(1 / \sqrt{T})$ it must hold that $\kappa \leq d^{2}$.

Also note that for the property of sAIC of not giving weights to models with fewer than $p_{0}$ lags to hold, $\kappa / T \rightarrow 0$ suffices.

\section{A.4 Relation of sAIC and sBIC Weights}

For this section set $\kappa=1$. The following lemma states the obvious fact that the sBIC assigns more weight to models with fewer lags than the sAIC for $\bar{p}=2$. It can be generalized to show that

$$
\frac{\widehat{w}_{B I C, p}}{\widehat{w}_{B I C, p^{\prime}}}>\frac{\widehat{w}_{A I C, p}}{\widehat{w}_{A I C, p^{\prime}}},
$$

for any $p, p^{\prime} \in\{1, \ldots, \bar{p}\}$ with $p<p^{\prime}$,

Lemma 3.3. Let $\bar{p}=2$, $\left|\widehat{\boldsymbol{\Sigma}}_{p}\right|^{-T / 2}>0$ for $p=1,2$, and $T^{d^{2} / 2}>\exp \left(d^{2}\right)$. Then

$$
\widehat{w}_{B I C, 1}>\widehat{w}_{A I C, 1}, \quad \widehat{w}_{B I C, 2}<\widehat{w}_{A I C, 2} \text {. }
$$

Note that the assumption on the choice of $d$ and $T$ is no real constraint in empirical applications as it already holds for the case $d=14$ and $T=8$.

Proof. Define $a_{p}:=\left|\widehat{\boldsymbol{\Sigma}}_{p}\right|^{-T / 2}$, and $S_{x I C}:=\sum_{p=1}^{\bar{p}} \exp \left(-x I C_{p} / 2\right)$. Starting from the assumption of the lemma, we get:

$$
\begin{aligned}
T^{d^{2} / 2} & >\exp \left(d^{2}\right) \\
\Leftrightarrow & \frac{T^{-d^{2} / 2}}{T^{-d^{2}}}>\frac{\exp \left(-d^{2}\right)}{\exp \left(-2 d^{2}\right)} \\
\Leftrightarrow & \frac{\widehat{w}_{B I C, 1} \frac{S_{B I C}}{a_{1}}}{\widehat{w}_{B I C, 2} \frac{S_{B I C}}{a_{2}}}>\frac{\widehat{w}_{A I C, 1} \frac{S_{A I C}}{a_{1}}}{\widehat{w}_{A I C, 2} \frac{S_{A I C}}{a_{2}}}
\end{aligned}
$$




$$
\begin{array}{ll}
\Leftrightarrow & \frac{\widehat{w}_{B I C, 1}}{\widehat{w}_{B I C, 2}}>\frac{\widehat{w}_{A I C, 1}}{\widehat{w}_{A I C, 2}} \\
\Leftrightarrow & \frac{1-\widehat{w}_{B I C, 2}}{\widehat{w}_{B I C, 2}}>\frac{1-\widehat{w}_{A I C, 2}}{\widehat{w}_{A I C, 2}} \\
\Leftrightarrow & \frac{1}{\widehat{w}_{B I C, 2}}-1>\frac{1}{\widehat{w}_{A I C, 2}}-1,
\end{array}
$$

since the weights sum to one. Then the result follows.

\section{A.5 Asymptotic Validity of the Bootstrap Procedure}

We will now establish the asymptotic validity of the bootstrap of Section 3.4 by using arguments of Bose (1988), Kilian (1998a,b), and Lütkepohl (1990), which we only have to adjust slightly for the model averaging case.

Note that as mentioned in Section III.A of Kilian (1998b) the proof shows validity for the studentized estimator only. This means that when the estimator's bootstrapped variance is different from the true variance, the method may still perform poorly in small samples, for example.

First, assume that the true lag order $p_{0}$ is known. For this case Kilian (1998b) shows asymptotic validity, and we reiterate the arguments in this paragraph. Estimates of $\boldsymbol{\beta}$ are obtained via OLS. Then we can use Bose (1988) who shows that the difference between the Edgeworth expansion for the bootstrapped estimate $\widehat{\boldsymbol{\beta}}^{*}$ and a comparable expansion for $\widehat{\boldsymbol{\beta}}$ is $o\left(T^{-1 / 2}\right)$ almost surely: Let $\boldsymbol{\Sigma}_{\widehat{\boldsymbol{\beta}}}=\boldsymbol{\Omega}^{-1} \otimes \boldsymbol{\Sigma}_{\boldsymbol{u}}$ be the asymptotic variance of $\widehat{\boldsymbol{\beta}}$, and define $\boldsymbol{\Sigma}_{\widehat{\boldsymbol{\beta}}^{*}}, \boldsymbol{\Sigma}_{\widehat{\boldsymbol{\sigma}}}$, and $\boldsymbol{\Sigma}_{\widehat{\boldsymbol{\sigma}}^{*}}$ analogously. Terms like $\boldsymbol{\Sigma}_{\widehat{\boldsymbol{\beta}}}^{-1 / 2}$ denote the Choleski factors of the inverse of those variances. Then, for almost every sequence $\left\{\boldsymbol{y}_{t}\right\}$,

$$
\sup _{\boldsymbol{x}}\left|P^{*}\left(\sqrt{T} \boldsymbol{\Sigma}_{\widehat{\boldsymbol{\beta}}^{*}}^{-1 / 2}\left(\widehat{\boldsymbol{\beta}}^{*}-\widehat{\boldsymbol{\beta}}\right) \leq \boldsymbol{x}\right)-P\left(\sqrt{T} \boldsymbol{\Sigma}_{\widehat{\boldsymbol{\beta}}}^{-1 / 2}(\widehat{\boldsymbol{\beta}}-\boldsymbol{\beta}) \leq \boldsymbol{x}\right)\right|=o\left(T^{-1 / 2}\right),
$$

(here $\boldsymbol{x} \in \mathbb{R}^{d^{2} p_{0}}$ ) and by an analogous argument,

$$
\sup _{\boldsymbol{x}}\left|P^{*}\left(\sqrt{T} \boldsymbol{\Sigma}_{\widehat{\boldsymbol{\sigma}}^{*}}^{-1 / 2}\left(\widehat{\boldsymbol{\sigma}}^{*}-\widehat{\boldsymbol{\sigma}}\right) \leq \boldsymbol{x}\right)-P\left(\sqrt{T} \boldsymbol{\Sigma}_{\widehat{\boldsymbol{\sigma}}}^{-1 / 2}(\widehat{\boldsymbol{\sigma}}-\boldsymbol{\sigma}) \leq \boldsymbol{x}\right)\right|=o\left(T^{-1 / 2}\right),
$$

(here $\boldsymbol{x} \in \mathbb{R}^{d(d+1) / 2}$ ). Further, it is well known that

$$
\sqrt{T}\left[\begin{array}{l}
\widehat{\boldsymbol{\beta}}-\boldsymbol{\beta} \\
\widehat{\boldsymbol{\sigma}}-\boldsymbol{\sigma}
\end{array}\right] \stackrel{d}{\longrightarrow} \mathcal{N}\left(\mathbf{0},\left[\begin{array}{cc}
\boldsymbol{\Sigma}_{\widehat{\boldsymbol{\beta}}} & \mathbf{O} \\
\mathbf{O} & \boldsymbol{\Sigma}_{\widehat{\boldsymbol{\sigma}}}
\end{array}\right]\right) .
$$

To make the dependence of the impulse response coefficient on the underlying model parameters explicit we will write $\theta(\boldsymbol{\beta}, \boldsymbol{\sigma})$ in the next few lines for this continuously differentiable function. Then its gradient $\nabla \theta(\boldsymbol{\beta}, \boldsymbol{\sigma})$ is a continuous function in its arguments $\boldsymbol{\beta}, \boldsymbol{\sigma}$. Now, by the Delta method we know

$$
\sqrt{T}(\theta(\widehat{\boldsymbol{\beta}}, \widehat{\boldsymbol{\sigma}})-\theta(\boldsymbol{\beta}, \boldsymbol{\sigma})) \stackrel{d}{\longrightarrow} \mathcal{N}(\underbrace{\mathbf{0}, \underbrace{\nabla \theta(\boldsymbol{\beta}, \boldsymbol{\sigma})^{\prime}\left[\begin{array}{cc}
\boldsymbol{\Sigma}_{\widehat{\boldsymbol{\beta}}} & \mathbf{O} \\
\mathbf{O} & \boldsymbol{\Sigma}_{\widehat{\boldsymbol{\sigma}}}
\end{array}\right] \nabla \theta(\boldsymbol{\beta}, \boldsymbol{\sigma})})}_{=: \sigma_{\widehat{\theta}}^{2}})
$$




\section{CHAPTER 3}

$$
\Rightarrow \sqrt{T} \sigma_{\widehat{\theta}}^{-1}(\theta(\widehat{\boldsymbol{\beta}}, \widehat{\boldsymbol{\sigma}})-\theta(\boldsymbol{\beta}, \boldsymbol{\sigma})) \stackrel{d}{\longrightarrow} \mathcal{N}(0,1),
$$

The same arguments hold in the bootstrap world so that Equation (3.16) analogously holds for the bootstrapped estimator.

Thus we have shown that the bootstrap yields an asymptotically valid approximation of the estimate of $\theta$, if the true lag order $p_{0}$ is known. So reintroducing $p_{0}$ as a subscript with the obvious notations we have shown:

$$
\sup _{x}\left|P^{*}\left(\sqrt{T} \sigma_{\widehat{\theta}_{p_{0}}^{*}}^{-1}\left(\widehat{\theta}_{p_{0}}^{*}-\widehat{\theta}_{p_{0}}\right) \leq x\right)-P\left(\sqrt{T} \sigma_{\widehat{\theta}_{p_{0}}}^{-1}\left(\widehat{\theta}_{p_{0}}-\theta\right) \leq x\right)\right|=O\left(T^{-1 / 2}\right) .
$$

These have been the arguments of Kilian (1998b) proving validity for the case of a known lag order.

Now we consider the model averaging case with sBIC weights. To shorten notation we will use $\widehat{\theta}=\widehat{\theta}\left(\widehat{\boldsymbol{w}}_{B I C}\right), \widehat{\theta}^{*}=\widehat{\theta}^{*}\left(\widehat{\boldsymbol{w}}_{B I C}^{*}\right)$. Define random variables

$$
\begin{aligned}
\widehat{\gamma} & =\left|\sqrt{T}(\widehat{\theta}-\theta)-\sqrt{T}\left(\widehat{\theta}_{p_{0}}-\theta\right)\right|, \\
\widehat{\gamma}^{*} & =\left|\sqrt{T}\left(\widehat{\theta}^{*}-\widehat{\theta}\right)-\sqrt{T}\left(\widehat{\theta}_{p_{0}}^{*}-\widehat{\theta}_{p_{0}}\right)\right| .
\end{aligned}
$$

Then by Theorem 3.2 for any $\delta>0$ :

$$
\begin{aligned}
P(\widehat{\gamma}>\delta) & \rightarrow 0, \\
P^{*}\left(\widehat{\gamma}^{*}>\delta\right) & \rightarrow 0 .
\end{aligned}
$$

Here the second statement holds if Theorem 3.2 also holds for the bootstrapped quantitities. This is essentially the case when Equation (3.2) also holds for the bootstrapped quantitities, as this is the main assumption needed. By Bose (1988)'s Remark 3.10, Equation (3.11) this can be seen to be the case.

Denote by $\sigma_{\widehat{\theta}}$ and $\sigma_{\widehat{\theta}^{*}}$ the variances of $\widehat{\theta}, \widehat{\theta}^{*}$ respectively. These are finite. Now let $T \in \mathbb{N}$. In the following we divide the probability space into the sets $\widehat{\gamma}>T^{-1}$, $\widehat{\gamma}^{*}>T^{-1}$ and their complements, and write,

$$
\begin{aligned}
& \sup _{x}\left|P^{*}\left(\sqrt{T} \sigma_{\widehat{\theta}^{*}}^{-1}\left(\widehat{\theta}^{*}-\widehat{\theta}\right) \leq x\right)-P\left(\sqrt{T} \sigma_{\widehat{\theta}}^{-1}(\widehat{\theta}-\theta) \leq x\right)\right| \\
= & \sup _{x} \mid P^{*}\left(\sqrt{T} \sigma_{\widehat{\theta}^{*}}^{-1}\left(\widehat{\theta}^{*}-\widehat{\theta}\right) \leq x, \widehat{\gamma}^{*} \leq T^{-1}\right)+P^{*}\left(\sqrt{T} \sigma_{\widehat{\theta}^{*}}^{-1}\left(\widehat{\theta}^{*}-\widehat{\theta}\right) \leq x, \widehat{\gamma}^{*}>T^{-1}\right) \\
& -P\left(\sqrt{T} \sigma_{\widehat{\theta}}^{-1}(\widehat{\theta}-\theta) \leq x, \widehat{\gamma} \leq T^{-1}\right)-P\left(\sqrt{T} \sigma_{\widehat{\theta}}^{-1}(\widehat{\theta}-\theta) \leq x, \widehat{\gamma}>T^{-1}\right) \mid \\
= & \sup _{x} \mid P^{*}\left(\sqrt{T} \sigma_{\widehat{\theta}^{*}}^{-1}\left(\widehat{\theta}^{*}-\widehat{\theta}\right) \leq x, \widehat{\gamma}^{*} \leq T^{-1}\right) \\
& +P^{*}\left(\sqrt{T} \sigma_{\widehat{\theta}^{*}}^{-1}\left(\widehat{\theta}^{*}-\widehat{\theta}\right) \leq x \mid \widehat{\gamma}^{*}>T^{-1}\right) P^{*}\left(\widehat{\gamma}^{*}>T^{-1}\right) \\
& -P\left(\sqrt{T} \sigma_{\widehat{\theta}}^{-1}(\widehat{\theta}-\theta) \leq x, \widehat{\gamma} \leq T^{-1}\right) \\
& -P\left(\sqrt{T} \sigma_{\widehat{\theta}}^{-1}(\widehat{\theta}-\theta) \leq x \mid \widehat{\gamma}>T^{-1}\right) P\left(\widehat{\gamma}>T^{-1}\right) \mid
\end{aligned}
$$


where the last equality simply follows by the definition of conditional probabilities. By the triangle inequality we continue

$$
\begin{aligned}
& \leq \sup _{x}\left|P^{*}\left(\sqrt{T} \sigma_{\widehat{\theta}^{*}}^{-1}\left(\widehat{\theta}^{*}-\widehat{\theta}\right) \leq x, \widehat{\gamma}^{*} \leq T^{-1}\right)-P\left(\sqrt{T} \sigma_{\widehat{\theta}}^{-1}(\widehat{\theta}-\theta) \leq x, \widehat{\gamma} \leq T^{-1}\right)\right| \\
& \quad+\sup _{x}\left|P^{*}\left(\sqrt{T} \sigma_{\widehat{\theta}^{*}}^{-1}\left(\widehat{\theta}^{*}-\widehat{\theta}\right) \leq x \mid \widehat{\gamma}^{*}>T^{-1}\right) P^{*}\left(\widehat{\gamma}^{*}>T^{-1}\right)\right| \\
& \quad+\sup _{x}\left|P\left(\sqrt{T} \sigma_{\widehat{\theta}}^{-1}(\widehat{\theta}-\theta) \leq x \mid \widehat{\gamma}>T^{-1}\right) P\left(\widehat{\gamma}>T^{-1}\right)\right| \\
& =\sup _{x}\left|P^{*}\left(\sqrt{T} \sigma_{\widehat{\theta}^{*}}^{-1}\left(\widehat{\theta}^{*}-\widehat{\theta}\right) \leq x, \widehat{\gamma}^{*} \leq T^{-1}\right)-P\left(\sqrt{T} \sigma_{\widehat{\theta}}^{-1}(\widehat{\theta}-\theta) \leq x, \widehat{\gamma} \leq T^{-1}\right)\right| \\
& \quad+\sup _{x}\left|P^{*}\left(\sqrt{T} \sigma_{\widehat{\theta}^{*}}^{-1}\left(\widehat{\theta}^{*}-\widehat{\theta}\right) \leq x \mid \widehat{\gamma}^{*}>T^{-1}\right)\right| \underbrace{P^{*}\left(\widehat{\gamma}^{*}>T^{-1}\right)}_{\rightarrow 0 \text { by Eqn (3.20) }} \\
& \quad+\sup _{x}\left|P\left(\sqrt{T} \sigma_{\widehat{\theta}}^{-1}(\widehat{\theta}-\theta) \leq x \mid \widehat{\gamma}>T^{-1}\right)\right| \underbrace{P\left(\widehat{\gamma}>T^{-1}\right)}_{\rightarrow 0 \text { by Eqn (3.19) }},
\end{aligned}
$$

where the last two lines converge to 0 since probabilities are bounded. So in the limit we are left with

$$
\begin{aligned}
& \lim _{T \rightarrow \infty} \sup _{x} \mid P^{*}\left(\sqrt{T} \sigma_{\widehat{\theta}^{*}}^{-1}\left(\widehat{\theta}^{*}-\widehat{\theta}\right) \leq x, \widehat{\gamma}^{*} \leq T^{-1}\right) \\
& -P\left(\sqrt{T} \sigma_{\widehat{\theta}}^{-1}(\widehat{\theta}-\theta) \leq x, \widehat{\gamma} \leq T^{-1}\right) \mid \\
= & \sup _{x} \mid \lim _{T \rightarrow \infty} P^{*}\left(\sqrt{T} \sigma_{\widehat{\theta}^{*}}^{-1}\left(\widehat{\theta}^{*}-\widehat{\theta}\right) \leq x, \widehat{\gamma}^{*} \leq T^{-1}\right) \\
& -\lim _{T \rightarrow \infty} P\left(\sqrt{T} \sigma_{\widehat{\theta}}^{-1}(\widehat{\theta}-\theta) \leq x, \widehat{\gamma} \leq T^{-1}\right) \mid \\
= & \sup _{x}\left|P^{*}\left(\sqrt{T} \sigma_{\widehat{\theta}_{p_{0}}^{*}}^{-1}\left(\widehat{\theta}_{p_{0}}^{*}-\widehat{\theta}_{p_{0}}\right) \leq x\right)-P\left(\sqrt{T} \sigma_{\widehat{\theta}_{p_{0}}}^{-1}\left(\widehat{\theta}_{p_{0}}-\theta\right) \leq x\right)\right|,
\end{aligned}
$$

because

$$
\begin{array}{r}
\bigcap_{T \in \mathbb{N}}\left\{\sqrt{T} \sigma_{\widehat{\theta}}^{-1}(\widehat{\theta}-\theta) \leq x,\left|\sqrt{T}(\widehat{\theta}-\theta)-\sqrt{T}\left(\widehat{\theta}_{p_{0}}-\theta\right)\right| \leq T^{-1}\right\} \\
=\left\{\sqrt{T} \sigma_{\widehat{\theta}_{p_{0}}}^{-1}\left(\widehat{\theta}_{p_{0}}-\theta\right) \leq x\right\},
\end{array}
$$

an analogous statement for the bootstrapped quantities holds, and because probability measures are antitonic continuous. Now, this is the expression that we bounded in probability in Equation (3.17). Thus, expression (3.21) is bounded. So we have shown asymptotic validity of the sBIC averaged estimator for both, the endogenous and exogenous bootstrap.

Now consider the case of using AIC. This case we cannot directly relate to the case of selecting the true lag order, because the sAIC-based averaged estimator has a larger variance. But by Equation (3.8), asymptotically AIC does not underselect, and in the large models the VAR coefficient estimates for $p>p_{0}$ tend to 


\section{CHAPTER 3}

zero: $\boldsymbol{A}_{p} \stackrel{p}{\longrightarrow}$ O. This means that the bootstrapped data will adequately reflect the observed data. Hence, covariances up to order $\bar{p}, \mathrm{E}\left(\boldsymbol{y}_{t} \boldsymbol{y}_{t-\bar{p}}^{\prime}\right)$, will be bootstrapped correctly. The sAIC-based averaged estimator will not assign any weight to models that are too small, thus is bounded in the limit, cf. Theorem 3.2, and its distribution gets bootstrapped correctly.

\section{A.6 Additional Simulation Results}

Figure 3.9 amends Figure 3.13 by showing results for all impulse response functions' estimates from the setting of Section 3.A.7.

Figures 3.10 and 3.11 supplement the results of Figures 3.2 and 3.5, respectively. They show the MSE alongside the squared bias, which, together with an estimator's variance, sums to the former. We see that while the performance of the sAIC-based estimator is virtually only governed by the estimator's variance, for the sBIC-based estimator the squared bias contribution is much more important.

This is why to explain the sAIC-based estimator's MSE performance, we inspect variances in Figure 3.12. The estimator labeled $\mathrm{fw}$ is the fixed weight estimator employing the weight vector $(0.11,0.10,0.3,0.52,0.13,0.6,0.3,0.2)$. These are the observed weights of the sAIC estimator of Table 3.1. Consequently, the fixed weight estimator in this case is the counterpart to the sAIC estimator but without estimation uncertainty of the weights. The effects of added variability and covariability (between weights and impulse response estimates) from estimating the weights appears to be strictly positive in total: The yellow line always lies below the blue broken line. The MSE plots for VAR(4) and the sAIC estimator of Figure 3.2 are very similar to the variance plots. We believe that the fact that the AIC- and sAIC- based estimators give an importance (selection frequency and weight, respectively, cf. Table 3.1) of around $10 \%$ to the models with 1 and 2 lags, and these models in turn have a much lower variance than the models with more lags, explains most of the MSE behavior of impulse response $1 \rightarrow 1$ at large horizons in Figure 3.2.

\section{A.7 Simulation Setting Imitating Stock and Watson (2001) Data}

In order to check our results with a second type of process we simulate data based on the estimates of Stock and Watson (2001). Note that we discuss this data set more in detail in Chapter 4. Stock and Watson (2001) estimate a VAR(4) for three macroeconomic variables: The unemployment rate, the federal funds rate, and the inflation rate. The frequency is quarterly, the largest estimated root is 0.97 at a sample size of 160 . Based on the estimates that we acquire from this specification we simulate 1000 Monte Carlo repetitions. In this Monte Carlo setting $p_{0}=4$ is the true lag order. Again we allow models up to $\bar{p}=12$, the largest integer smaller than $\sqrt{T}$, to be estimated. Differently from the MSE graphs of the previous subsection, here we divide the MSE of the AIC-based impulse response estimators by the MSE of the true lag method to obtain a sort of normalization making more evident the differences in MSE. This is similar to the normalization used 

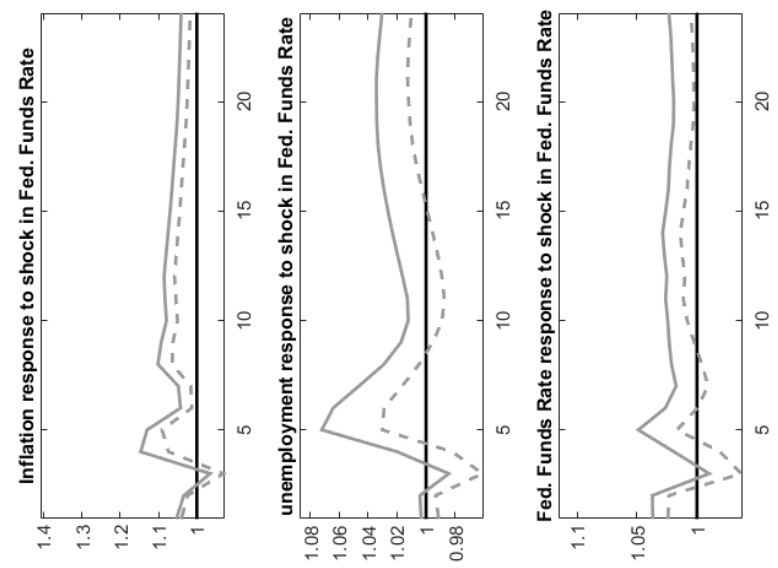

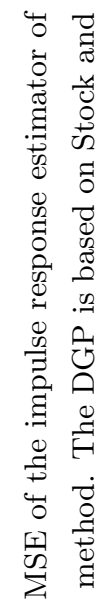
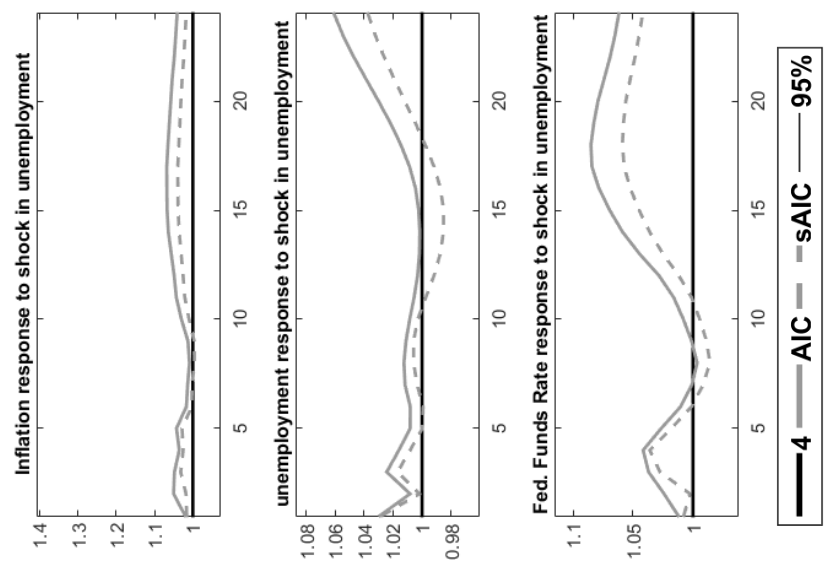

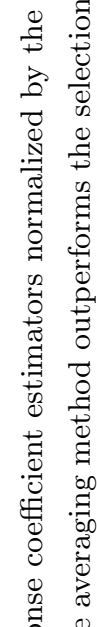
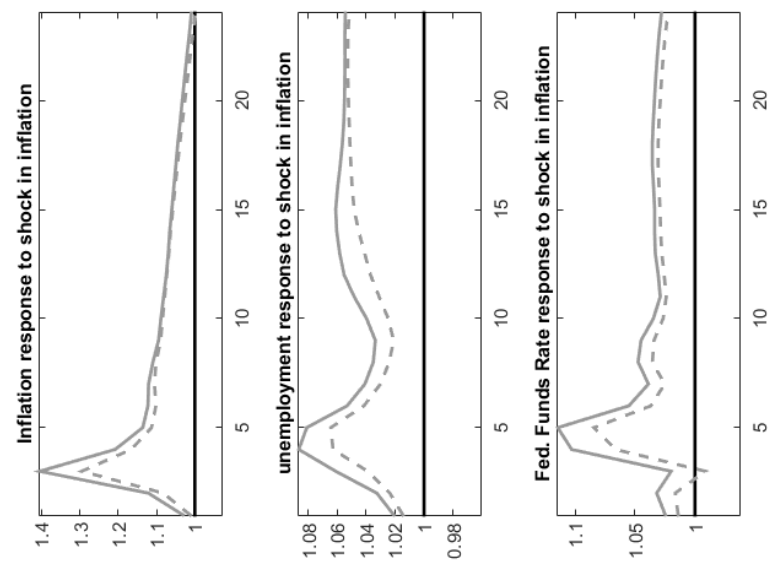

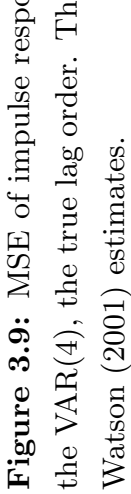




\section{CHAPTER 3}
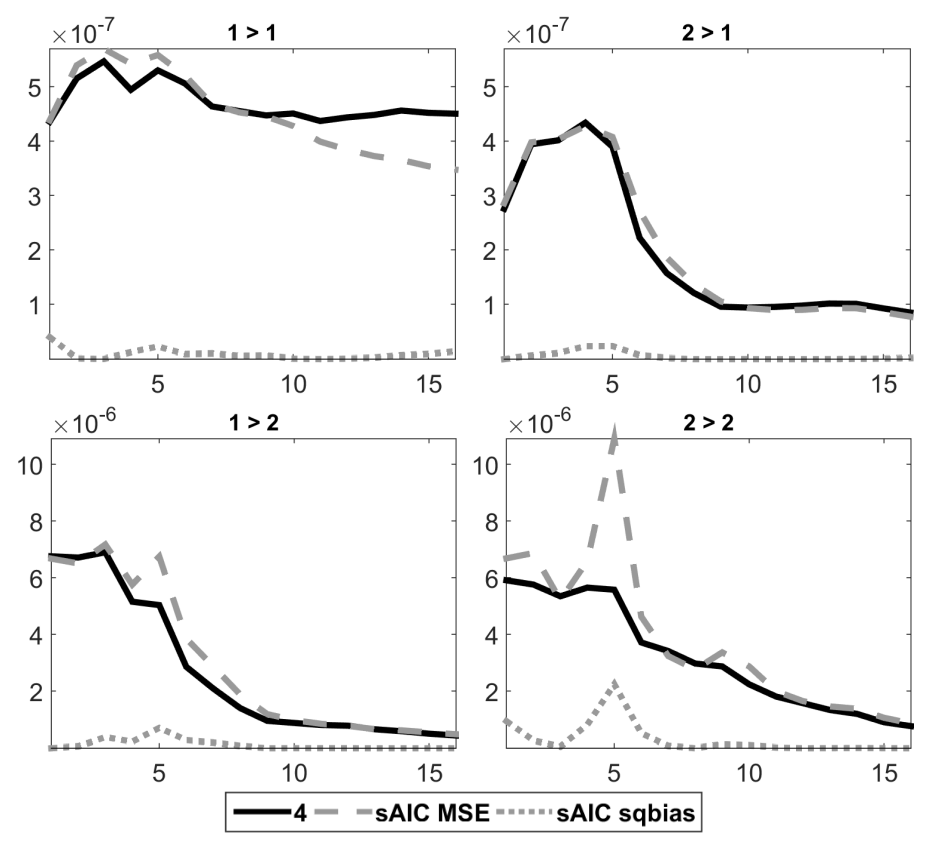

Figure 3.10: MSE and squared bias of sAIC-based estimator. The response of variable $j$ to a shock in variable $i$ is labeled $i>j . \mathrm{T}=80$.

by Hansen (2007) for plotting. Figure 3.13 shows the normalized MSE of these different impulse response coefficient estimators. The sAIC-based estimator has a lower MSE than the AIC-based selection estimator. The same holds true for the other impulse response coefficient estimates, which are shown in the appendix, Figure 3.9.

Figure 3.14 shows estimated impulse response functions for one particular simulation run together with their bootstrapped confidence intervals. While AIC suggests a lag of 6 , the sAIC based estimator divides the weights across all available lags. As a consequence the resulting impulse response functions are different from each other, and we see that the $66 \%$ confidence interval around the AICbased function barely contains the sAIC-based function at horizon 6. For lower significance levels the sAIC function might not be included in the intervals at all, while it is one desired property of confidence intervals to contain the estimate. This illustrates the natural requirement of confidence intervals which take into account the nature of the averaging estimator. We define such a procedure in Section 3.4 and prove its validity.

Figure 3.15 shows the coverage of the selection and averaging methods' pointwise confidence intervals, confirming the results of the previous section that both estimates lie close together. From these pointwise confidence intervals joint confidence bands of the adjusted Bonferroni method have been calculated and their effective coverages displayed in Table 3.4. From the table we see that none of ei- 

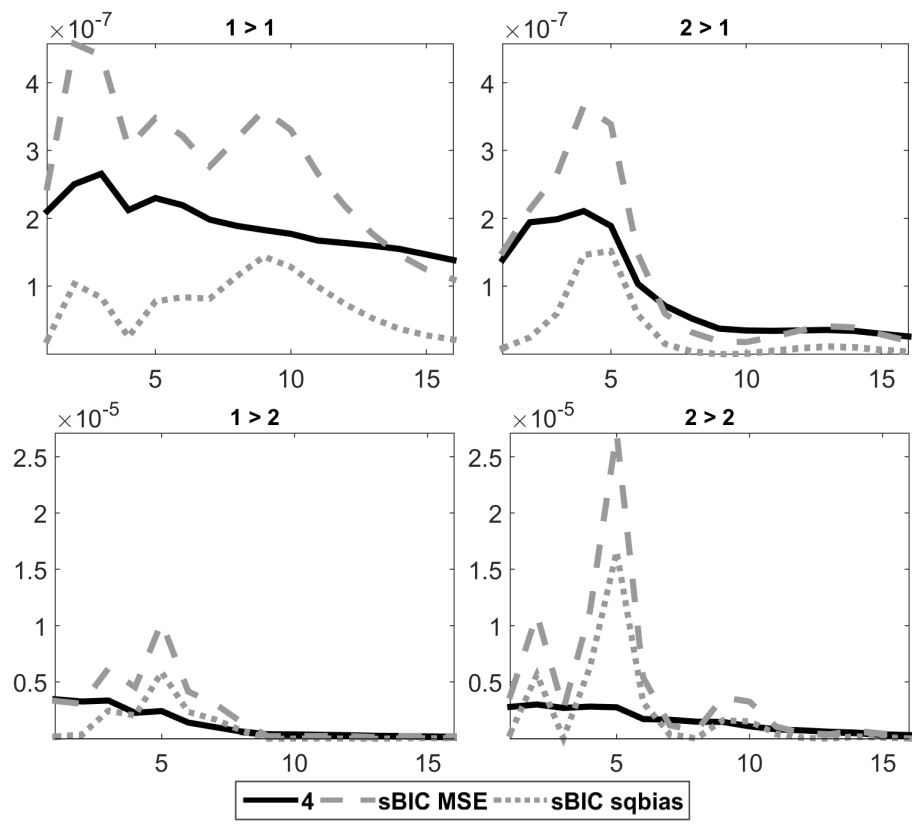

Figure 3.11: MSE and squared bias of sBIC-based estimator. The response of variable $j$ to a shock in variable $i$ is labeled $i>j$. T=160.

ther method uniformly outperforms the other in terms of having a coverage closer to the intended nominal coverage. The estimates on the Stock and Watson (2001) inspired data set hence confirm the observations on the MSE and effective coverage performance made in Section 3.5.1. 
CHAPTER 3
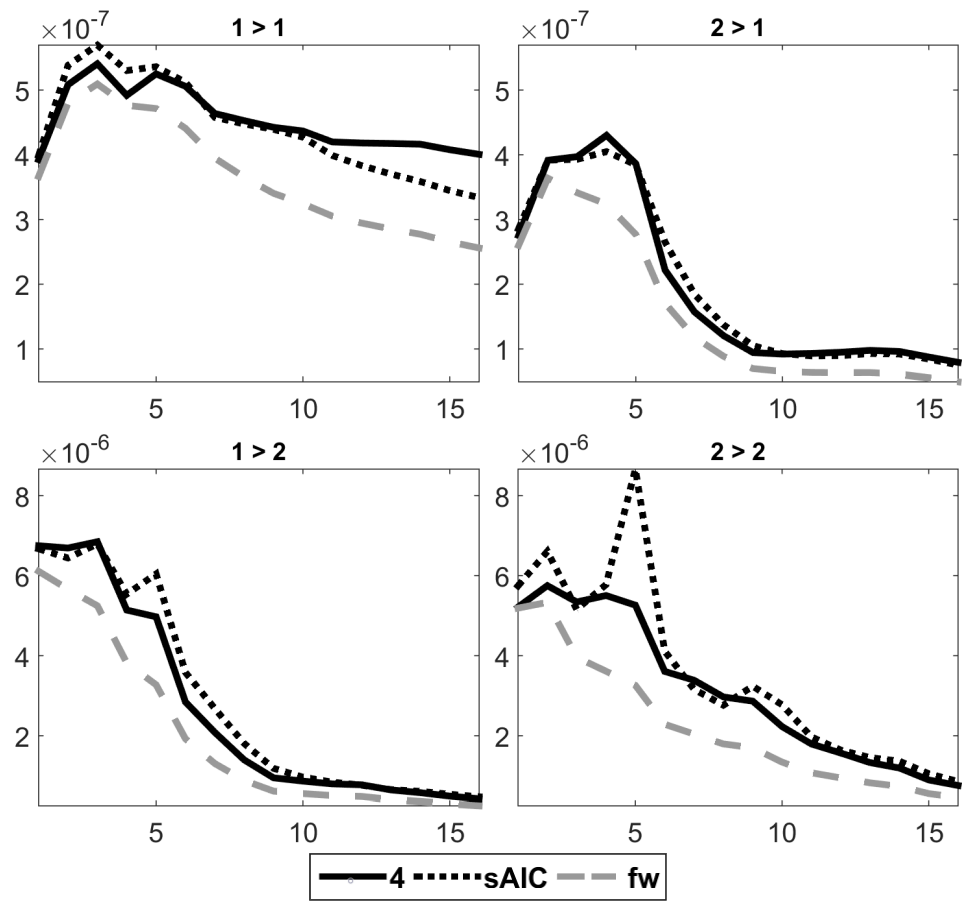

Figure 3.12: Empirical variances of the sAIC-based and the fixed weight estimator with weights equal to the average sAIC weights. The response of variable $j$ to a shock in variable $i$ is labeled $i>j$. T $=80$. 


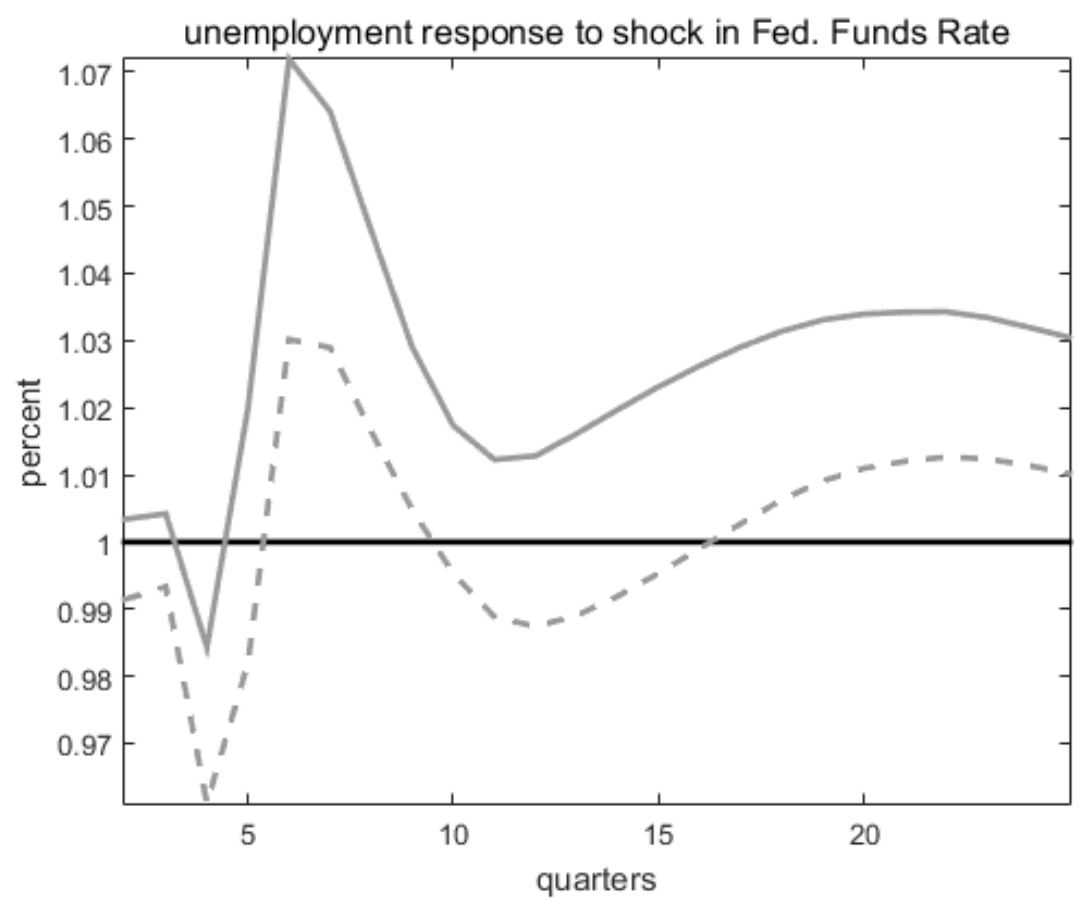

Figure 3.13: MSE of impulse response coefficient estimators normalized by the MSE of the impulse response estimator of the $\operatorname{VAR}(4)$, the true lag order. Black line: VAR(4). Gray solid: AIC. Gray broken: sAIC. The averaging method outperforms the selection method. The DGP is based on Stock and Watson (2001) estimates.

\section{A.8 Simulation Setting Based on Lütkepohl et al. (2015)}

In this section we consider a DGP where the larger of two sensible models is hard to detect. Such a setting might favour model averaging methods. We use the DGP defined in Lütkepohl et al. (2015), and amend it by a second coefficient matrix with only one non zero coefficient. With the notation of Section 3.2 above, let the $\operatorname{VAR}(2)$ DGP be defined by

$$
\boldsymbol{A}_{1}=\left(\begin{array}{cc}
a & 0 \\
0.5 & 0.5
\end{array}\right), \boldsymbol{A}_{2}=\left(\begin{array}{ll}
b & 0 \\
0 & 0
\end{array}\right), \boldsymbol{\Sigma}_{u}=\left(\begin{array}{cc}
1 & 0.3 \\
0.3 & 1
\end{array}\right) .
$$

We vary $a$ and $b$ between -1 and 1 under the constraint that the process remains stationary. Figures 3.16 - 3.19 show MSE results for some of these parameter combinations. Table 3.5 shows the effective coverage of the adjusted Bonferroni methods. We note that the averaging method outperforms the selection method in terms of MSE but not in terms of effective coverage. This goes in line with the observations that we made in the main text. Also, the effective coverage of the sAIC method is consistently lower than that of the AIC method. This brings the 


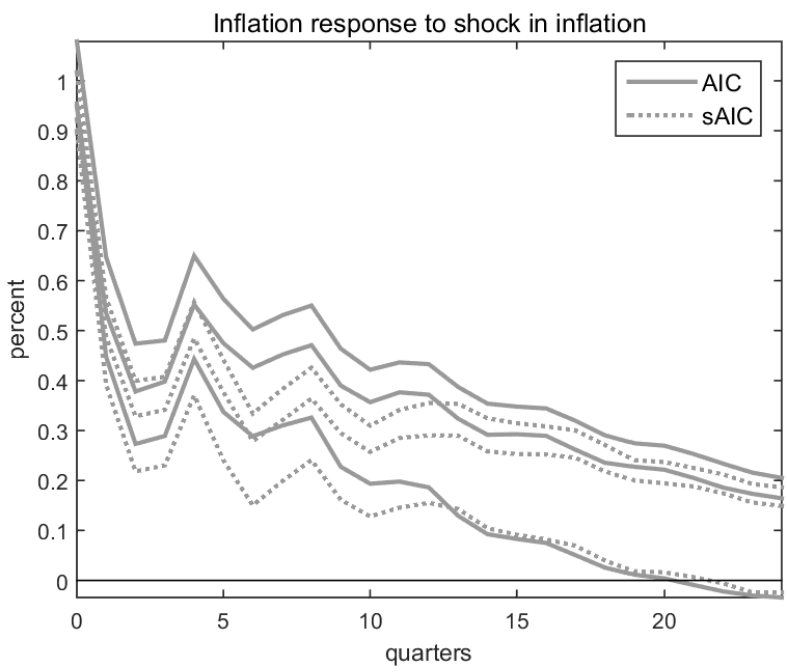

Figure 3.14: Impulse response of inflation to a shock in inflation. AICand sAIC-based estimates together with their $66 \%$ bootstrapped (pointwise) confidence intervals. The DGP is based on Stock and Watson (2001) estimates. $T=80$.

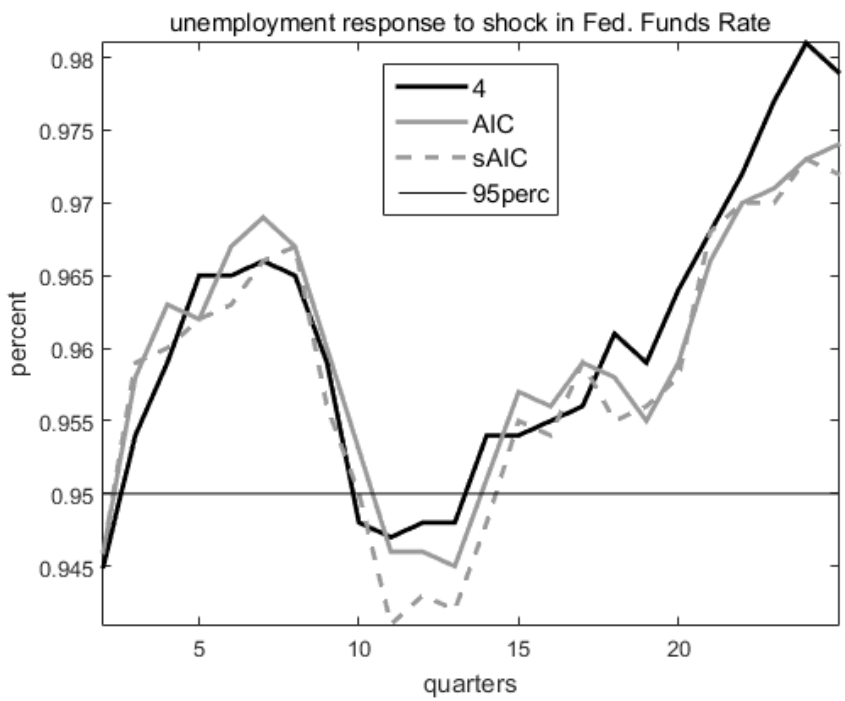

Figure 3.15: Coverage of impulse response coefficient estimators. Black line: VAR(4). Gray solid: AIC. Gray broken: sAIC. The DGP is based on Stock and Watson (2001) estimates. 


\section{A. APPENDIX}

\begin{tabular}{cc|l|ccccccccc}
$\bar{p}$ & cov & mthd $\backslash$ IR & $1 \rightarrow 1$ & $2 \rightarrow 1$ & $3 \rightarrow 1$ & $1 \rightarrow 2$ & $2 \rightarrow 2$ & $3 \rightarrow 2$ & $1 \rightarrow 3$ & $2 \rightarrow 3$ & $3 \rightarrow 3$ \\
\hline \multirow{2}{*}{9} & \multirow{2}{*}{95} & true lag & 87.7 & 92.9 & 92.5 & 92.0 & 83.1 & 94.6 & 91.2 & 90.2 & 88.4 \\
& & AIC & 86.2 & 94.0 & 91.7 & 92.0 & 82.1 & 94.7 & 91.2 & 89.2 & 86.7 \\
& & sAIC & 85.7 & 93.6 & 90.9 & 91.7 & 81.6 & 94.4 & 90.6 & 89.8 & 86.5 \\
\hline \multirow{2}{*}{9} & 66 & true lag & 60.5 & 67.3 & 64.5 & 64.0 & 53.0 & 64.5 & 64.8 & 63.8 & 54.0 \\
& & AIC & 57.3 & 65.8 & 65.5 & 65.0 & 53.3 & 66.0 & 64.5 & 62.3 & 53.5 \\
& & sAIC & 57.3 & 66.8 & 66.0 & 65.3 & 53.0 & 65.5 & 64.8 & 61.0 & 54.5 \\
\hline
\end{tabular}

Table 3.4: Effective coverage (in percent) of the adjusted Bonferroni joint confidence bands for different estimators (methods/mthd). $T=160$. Top panel: $\bar{p}=12$, nominal coverage (cov): $95 \%$. Bottom panel: $\bar{p}=9$, nominal coverage (cov): $66 \%$.

effective coverage of the former compared to the latter closer to the desired $95 \%$ coverage for the estimation of the response of variable 2 to a shock in variable 1 with $H=16$ horizons, $a=0, b=0.05$, for example, but further away for $a=0, b=0.95$.
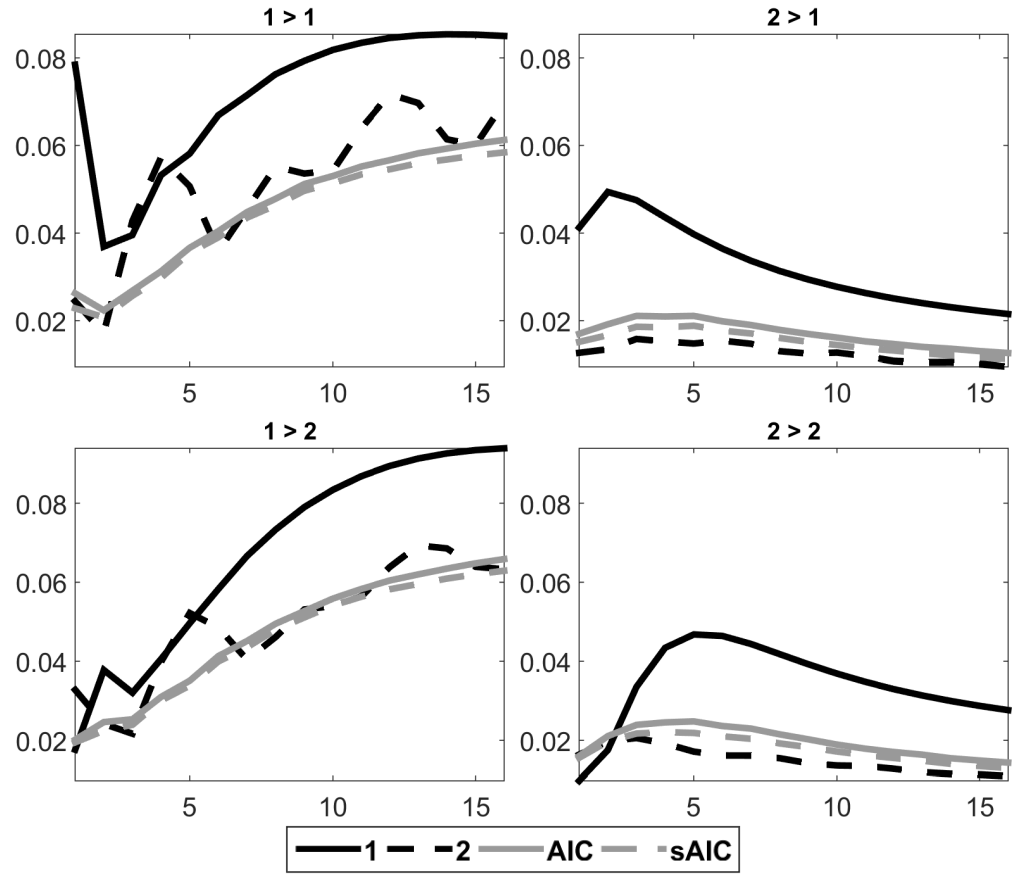

Figure 3.16: MSE of impulse response coefficient estimators. The response of variable $j$ to a shock in variable $i$ is labeled $i>j$. The DGP is defined by Equations 3.22 with $a=0.5, b=0.45, T=80$. The averaging method outperforms the selection method. 

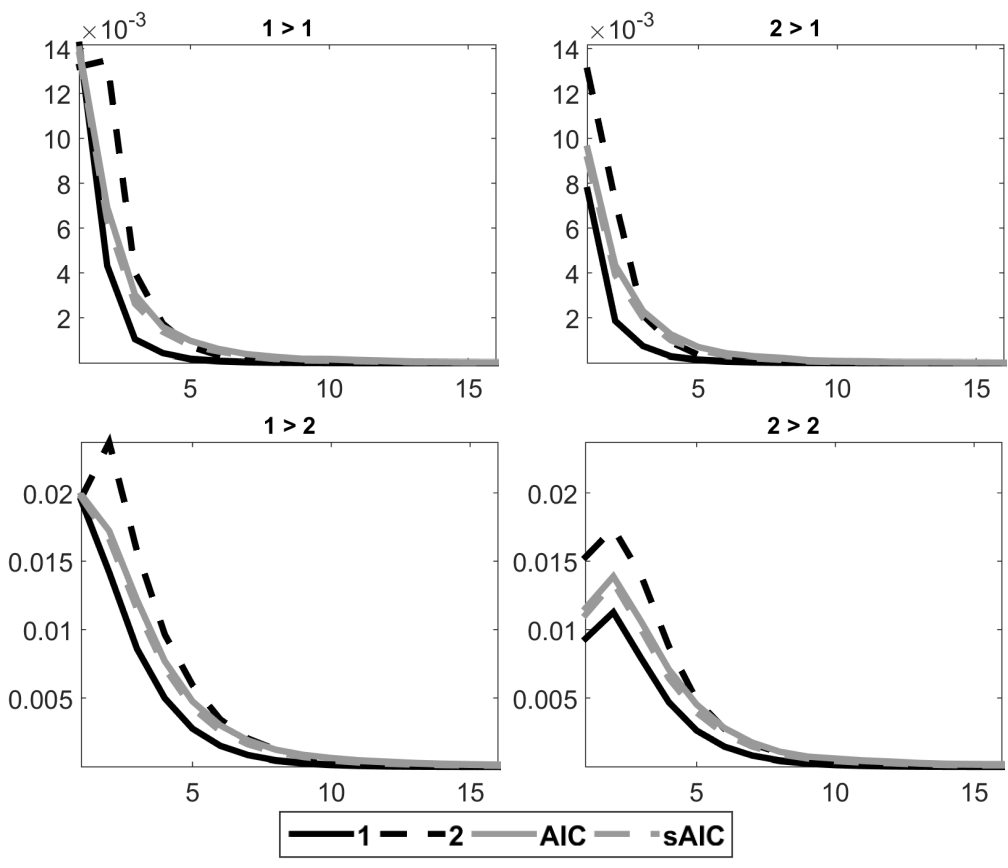

Figure 3.17: MSE of impulse response coefficient estimators. The response of variable $j$ to a shock in variable $i$ is labeled $i>j$. The DGP is defined by Equations 3.22 with $a=0.0, b=0.05, T=80$. The averaging method outperforms the selection method.

\begin{tabular}{l|llll|cccc}
$\mathrm{H}$ & method & $T$ & $a$ & $b \backslash \mathrm{IR}$ & $1 \rightarrow 1$ & $2 \rightarrow 1$ & $1 \rightarrow 2$ & $2 \rightarrow 2$ \\
\hline \multirow{2}{*}{16} & AIC & 80 & 0.5 & 0.45 & 61.6 & 98.0 & 65.0 & 91.2 \\
& AIC & 80 & 0.0 & 0.05 & 96.2 & 99.6 & 97.1 & 93.1 \\
& AIC & 80 & 0.0 & 0.95 & 63.4 & 98.6 & 58.7 & 90.2 \\
\hline \multirow{2}{*}{16} & sAIC & 80 & 0.5 & 0.45 & 59.5 & 97.6 & 62.9 & 89.5 \\
& sAIC & 80 & 0.0 & 0.05 & 95.6 & 99.6 & 96.1 & 91.5 \\
& sAIC & 80 & 0.0 & 0.95 & 61.6 & 98.1 & 56.9 & 88.9 \\
\hline \multirow{2}{*}{10} & AIC & 100 & 0.0 & 0.05 & 92.2 & 99.0 & 92.6 & 93.3 \\
& sAIC & 100 & 0.0 & 0.05 & 90.7 & 98.8 & 90.4 & 92.2 \\
\hline
\end{tabular}

Table 3.5: Effective coverage (in percent) of the nominal $95 \%$ adjusted Bonferroni joint confidence bands. $\mathrm{H}$ denotes the number of horizons of the estimated impulse response function, $\mathrm{T}$ the sample size, $\mathrm{a}$ and $\mathrm{b}$ denote parameter values of the simulated DGP. 

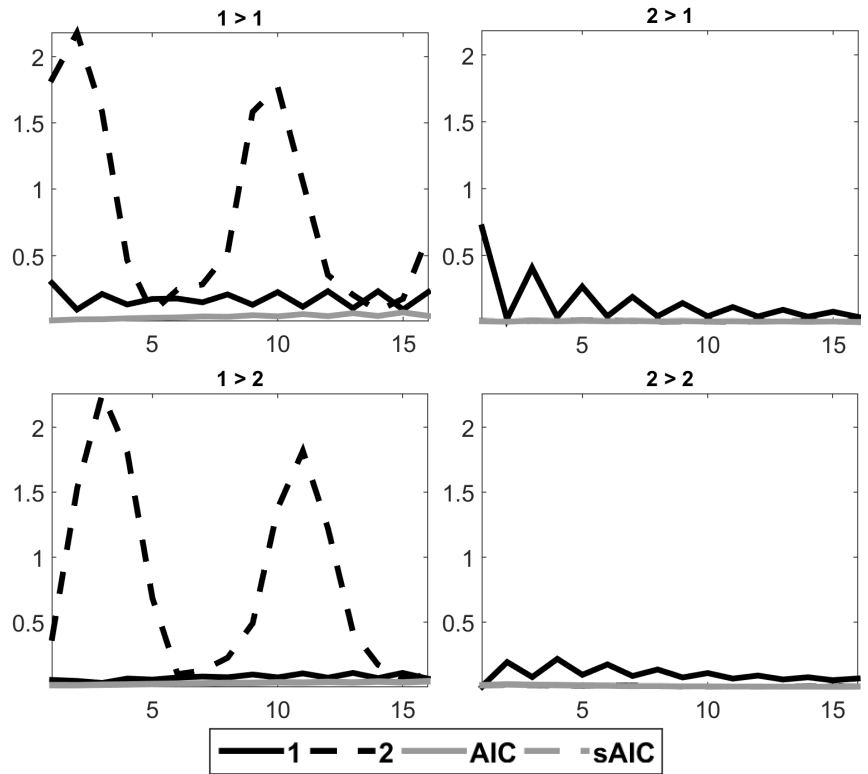

Figure 3.18: MSE of impulse response coefficient estimators. The response of variable $j$ to a shock in variable $i$ is labeled $i>j$. The DGP is defined by Equations 3.22 with $a=0.0, b=0.95, T=80$. The averaging method outperforms the selection method.
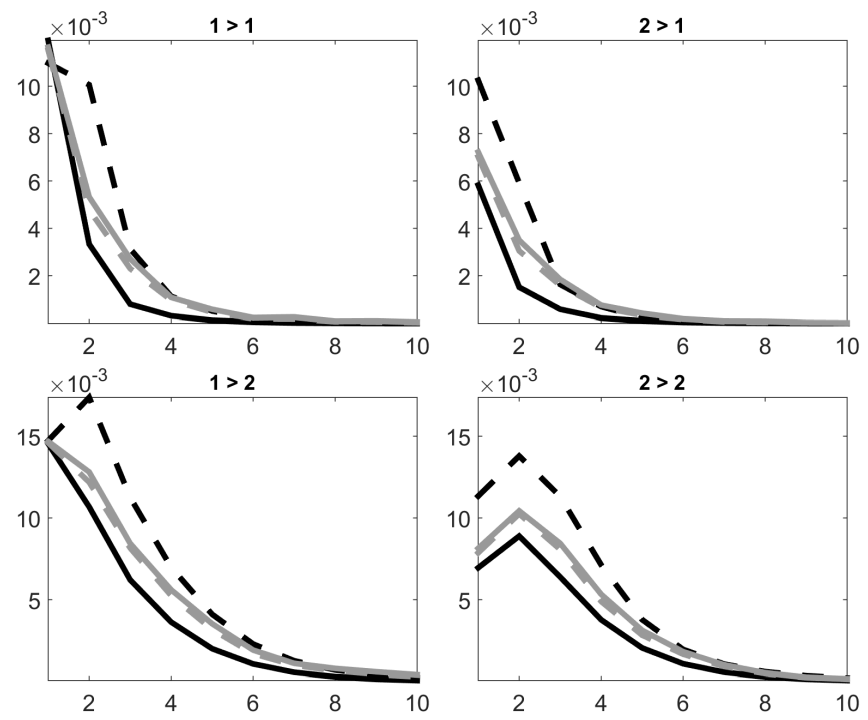

Figure 3.19: Like Figure 3.18, but with $a=0.0, b=0.05, T=100$. 


\section{Chapter 4}

\section{An Application of Model Averaging in Structural VARs}

Structural VAR models are a central tool in macroeconomic and monetary policy analysis. We make suggestions on modelling choices by revisiting previous empirical studies using VAR models and applying model selection and model averaging criteria, that have not been previously applied in this setting. Among other things we find that AIC-based model averaging yields smoother and more precise estimates of impulse response functions than AIC-based model selection, and that sampling data at a higher frequency (monthly) leads to higher precision than sampling at lower frequencies. At the same time, the results drawn from lower frequency (quarterly) data are more robust to the choice of model selection method.

\subsection{Introduction}

All the estimators that were presented in the previous chapters together with their theoretical properties, are applicable in structural VAR modeling. The question that follows is how do they perform with empirical data? This chapter answers this question by revisiting three empirical studies that use VARs for analyzing monetary policy. The chapter gives guidance on the use of these estimators to empirical researchers who amend their toolbox with these. Additionally to applying the estimators from the previous chapters, we use the opportunity of applying the jackknife model averaging estimator, that is relatively new and has not been as widely used in this setting, and the equal weights average estimator, to empirical data, the latter of which interestingly performs similarly to the studies' benchmark estimates for quarterly data. We also add some other commonly used model selection criteria. Hence, we end up with a host of different model selection and model averaging estimators whose estimates we compare. This also serves as a kind of 
robustness check, as discussed in the following paragraph, answering the question of how robust the results drawn from our sVAR analyses are to the concrete choice of model selection or model averaging method. The analysis of the performance of the above-mentioned estimators in empirical macroeconomic modelling forms the main contribution of this chapter. The three studies we revisit are Christiano et al. (1999), Stock and Watson (2001), and Uhlig (2005), which have been carefully selected for their similar analyses. They all estimate exogenous monetary shocks for US data sets of similar time periods, and apply the same structural parametrization for identifying impulse response functions. But the data sets do differ in sampling frequency and number of variables, and this, additionally to our above-mentioned contribution, allows us to compare the performance of the estimators across the different sampling frequencies of the data sets and to make suggestions on sampling frequency based on a comparison of estimator precision across the different data sets.

Robustness checks on econometric results are important, because a researcher setting up a structural VAR analysis is confronted with model uncertainty at various stages. Consider the case of frequentist model selection. Here, after having decided on a set of variables to model, she needs to decide on a model size, i.e. lag order of the VAR, but before making this choice she first needs to settle on a method of choosing that model size (and a set of models to consider for selection). Out of a large set of such methods some typically used examples are model selection by minimizing some model selection criterion, and series of tests like likelihood ratio tests. In the case of model averaging the problem remains in essence the same. We will not treat the related Bayesian methods like Bayesian model averaging, as the previous studies, that we discuss in this chapter, as well as the results of the previous chapters, also rely on frequentist methods for estimation. Frequentist model selection and model averaging methods assign weights to the estimators of certain parameters of interest that result from using the different models in the set of models considered for selection or averaging. In the case of selection these weights are binary, either 0 or 1 , and only the estimates of one of the models receive weight 1 . In the case of averaging the weights are typically restricted to lie between 0 and 1 and sum to 1 . So we can think of the term estimator weighting methods to refer to both, model selection and model averaging methods. We check the robustness of the results of the three classical monetary sVAR studies by applying new model selection methods and model averaging methods that had not been as well researched or even known when those studies were authored. We compare the results to the ones in the original studies, and analyze what type of estimates the additional methods deliver.

Our idea is similar to that of Ivanov and Kilian (2005), who run a simulation study of different VAR models to identify suitable model selection criteria for different types of macroeconomic data (e.g. different frequencies of observations). Next to the model selection criteria AIC, BIC and HQ (Hannan-Quinn) they also include the methods of sequential likelihood ratio tests with and without bias correction, and sequential Lagrange multiplier tests. Ivanov and Kilian (2005) conclude that the lowest MSE of estimated impulse response functions can be achieved when using the model selection methods instead of sequential tests. 


\section{CHAPTER 4}

Specifically they recommend using the AIC for monthly macroeconomic data, and the HQ for quarterly data. Their results are an extension of Kilian (2001), who compares impulse responses' MSE's in a bivariate setting for a few different model selection criteria.

One of the new model selection criteria that we add to the analyses is the focused information criterion (FIC) that was pioneered by Claeskens and Hjort (2003). It has been used in a time series setting by Hansen (2005), Claeskens et al. (2007) and rigorously defined in Chapter 2. While many common information criteria are geared towards measuring the ability of a model to explain the observed data, the focused information criterion allows to measure the fit of (almost) any arbitrary parameter that is a function of the assumed model. We want to investigate whether this difference in object of interest leads to qualitatively different estimation results. Another set of methods that we add are frequentist model averaging methods. Model averaging has been advocated in Hansen (2005) as a means to ease the problem of model uncertainty. While Bayesian model averaging, which we do not make use of ${ }^{1}$, is an established estimation method, frequentist model averaging is a newer concept and has not been as widely applied yet. We show in Chapter 3 that the smooth averaging estimators based on AIC and BIC, sAIC, sBIC, do converge to their model selection counterparts. By sAICb we denote the sAIC estimator with the weight scaling parameter $\kappa$, described in Section 3.3.1 above and Section 4.2 below, set equal to $d^{2}$, with $d$ being the number of variables in the VAR. Hjort and Claeskens (2003a) establish properties of frequentist model averaging. We find that the sAICb method delivers results that are similar to, but more intuitive than those of the commonly used AIC method, and that one of the FIC averaging methods, in turn, performs similarly to the sAICb method. In terms of robustness we find that for the quarterly data the concrete choice of estimator weighting method is less important than for the monthly data.

In structural VAR analyses, additionally to the size of the model another choice to make is that of the orthogonalization of the impulse responses, which is a choice of parametrization from a class of empirically equivalent models, cf. Chapter 9 of Lütkepohl (2005). As in the previous chapters of this thesis and in the sVAR studies that we will discuss and use here, we use a recursive identification scheme, cf. Chapter 1. As the different available identification schemes of structural VARs are not the center of our analysis and in order to be able to compare our findings with those of the studies under consideration, we apply the same orderings of the variables as chosen in the studies. As alluded to above, the data sets differ in sampling frequency. While Uhlig (2005) uses a monthly data set, the other two articles rely on quarterly data. This offers an interesting opportunity for studying the impact that sampling macroeconomic data at different frequencies has on precision, i.e. the mean squared error. The data sets also differ in number of variables that are being modelled. We find that including more variables seems to worsen estimator precision. To quantify precision we use bootstrapped confidence bands and we find that sampling data at higher frequency increases estimator precision. The articles are first treated separately in Section 4.3, their results are

\footnotetext{
${ }^{1}$ We do apply the Bayesian information criterion, BIC, but within frequentist type model selection and averaging.
} 
then discussed in the subsequent part. All of this is preluded by a presentation of the methods that we apply.

In terms of notation $p$ stands for the minimum, and $\bar{p}$ for the maximum considered number of lags used in the estimated VAR model, whose number of variables is denoted by $d$. We consider the model set of $\operatorname{VAR}(p)$ models with $p \in\{\underline{p}, \ldots, \bar{p}\}$. Unless noted otherwise we have chosen $p=1$. In the following $T$ denotes the effective sample size, so the number of observations that remains when deducting the first $\bar{p}$ observations. We will often simply write responses when we mean estimated impulse responses.

\subsection{Estimation Methods}

VAR Modelling As mentioned in Chapter 1 and in Section 4.1 above, all three studies we consider here use a recursive ordering of the variables in the VAR to ensure identifiability of the impulse responses. Interestingly one of these, namely Uhlig (2005), contrasts two different approaches to identify the VAR's structural innovations, the second one of which is identification by sign restrictions. We do not discuss this concept in detail here, but give a brief summary of it in Chapter 1 .

We do find a largest absolute value of the lag polynomial's root close to 1 for all three specifications from these studies. And while it is also often conjectured in the literature that interest rate time series like the federal funds rate are not stationary, we follow the original authors in using the same data transformations even if the resulting estimates imply nonstationarity. So we assume that the VARs are stable. We do this to be able to directly compare our results with theirs. Specifically, the authors of the three studies do not mention having imposed any unit roots. We leave it for future research to elaborate on the effect of imposing a unit root in the specifications. Note that Paulsen (1984) shows that the BIC and the HQ information criterion remain consistent in the presence of unit roots.

Model Selection and Averaging Apart from plotting the impulse response estimates implied by the original authors' chosen lag order we use a host of different model selection and model averaging procedures to obtain estimates. We use methods that are already commonly applied, as well as more recent methods, that have not been as widely applied in structural VAR analyses. The new theoretical results for the FIC methods of Chapter 2 for example, demand an application to empirical data and a comparison with the findings for alternative models.

First of all we select models based on the well known Akaike and the Bayesian information criterion, AIC and BIC. These are described in Chapter 3 above together with their smoothed model averaging counterparts, sAIC and sBIC. The smoothed estimators depend on a weight scaling parameter $\kappa$. For $\kappa=1$ we obtain the case of the smoothed estimators as described in the literature to date, but for any $\kappa$ with $0<\kappa \leq d^{2}$, where $d$ is the number of variables in the VAR, we have shown in Section 3.3 above that the asymptotic properties typical for the AIC and the BIC estimator still hold. In our graphs we plot the sAIC estimators with $\kappa=1, d^{2}$ and label these with sAICa, sAICb respectively. The former has more 


\section{CHAPTER 4}

concentrated weights and is more similar to the AIC model selection estimator, cf. Section 3.3.1, while the latter has more spread out weights and is more similar to the averaging estimator that assigns equal weight of $1 / \bar{p}$ to each model, the equal weights estimator, labeled EW. Hansen (2008) calls this simple averaging in the context of forecast combinations. One might also be tempted to put the averaging estimator with $\kappa=T$, the sample size, to the test, as this estimator results from calculating smoothed weights from another commonly encountered definition of the AIC score, as discussed in Section 3.3.1. This averaging estimator delivers results very similar to the equal weights estimator. In fact, only for the data set of Christiano et al. (1999) are the estimated impulse response functions different between the equal weights estimator and the smoothed weights estimator with $\kappa=T$. But also for that case do they lie closely together and have very similar shapes.

Another model selection criterion that we include in our analysis because of its close relation to the AIC and the BIC is the Hannan-Quinn criterion, labeled HQ in the following. For the $p$ th candidate model, $p \in\{p, \ldots, \bar{p}\}$, the HQ score is defined as

$$
H Q_{p}=T \log \left|\widehat{\boldsymbol{\Sigma}}_{p}\right|+2 p d^{2} \log (\log (T)),
$$

cf. Equation (4.19) of Juselius (2006). Here $\widehat{\boldsymbol{\Sigma}}_{p}$ is an estimate of the covariance matrix of the disturbances in a model with $p$ lags. The Hannan-Quinn estimator is the one that selects the model with the lowest HQ score.

We will discuss the estimated impulse response functions for the different data sets and estimation procedures in detail in the coming sections, but provide Figure 4.1 here already as a first illustration. It shows the estimated impulse response of inflation to a shock in the federal funds rate resulting from using a VAR(4) and the other model selection and averaging methods discussed here for estimation. The green line shows the estimate resulting from employing BIC model selection. When using sBIC model averaging with $\kappa=1$ the same estimates are obtained in this case, which is why there is no separate line plotted. The line based on the AIC averaging estimator with $\kappa=d^{2}$, sAICb, lies closer to the one for equal weights than the sAICa implied estimate. For $\kappa=T$ we obtain the same estimates as with the equal weights estimator, which is why this line is not plotted.

The jackknife model averaging estimator is labeled JMA. It was suggested by Hansen and Racine (2012) for estimating linear models with heteroskedastic errors and is applied in the simulations of Chapter 2 above. Hansen and Racine (2012) show that the model weights obtained with the JMA estimator achieve the lowest possible expected squared error under certain conditions.

Furthermore we apply the FIC estimators developed in Chapter 2. These can be defined for different focus parameters, for example for one specific impulse response coefficient. This allows a different model to be selected at each horizon, and as Figure 4.1 shows this leads to very erratic estimates. The red line labeled FIC corresponds to this definition. It is the one showing the lowest point estimate at horizon 14 and the highest (at around 0) at horizon 18. Since one is typically interested in finding a model specification that estimates whole impulse response functions, we will not use this narrowly focused estimator in the following, but 


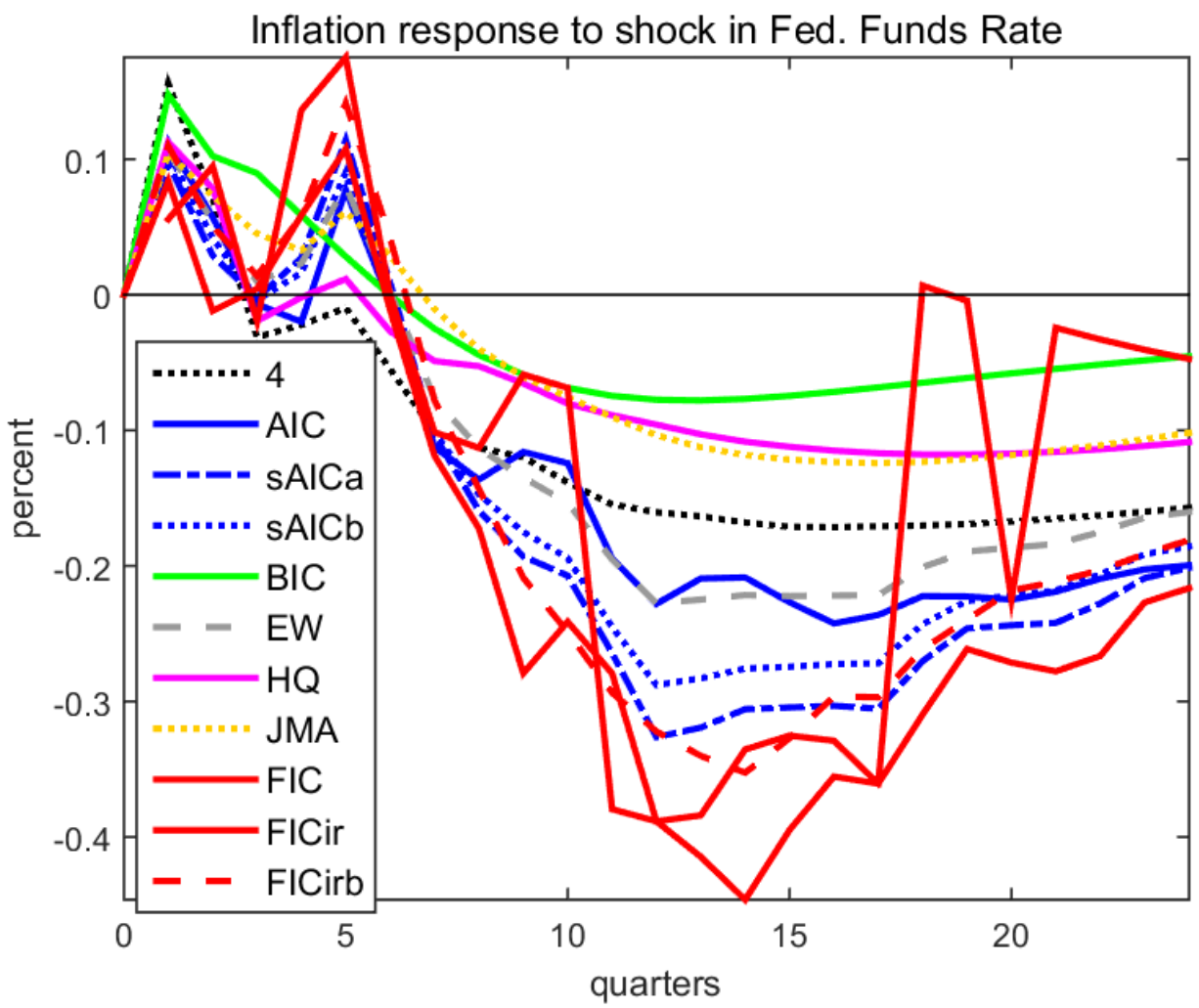

Figure 4.1: Stock and Watson (2001) data set IRs estimated from a VAR(4) (black line) and various estimation methods. $\bar{p}=12$.

define a broader focus, namely all the impulse response coefficients under consideration in the specific model. These are $d^{2}(H+1)$ in number, where $d$ is the number of variables in the VAR, and $H$ is the number of horizons under consideration (for Stock and Watson (2001) $H=24$, for example). We choose the trace of the estimated AMSE matrix, cf. Remark 2.10, as the means to aggregate the individual FIC scores, and label this method FICir. This means that the FIC is set up to select the model that minimizes the sum of the $d^{2}(H+1)$ estimated asymptotic MSE's of the impulse response coefficients. Additionally we consider the criterion's model averaging version without bias correction, cf. Equation (2.10), and label it FICirb. The definition of our focus is just one particular illustratory choice, but in general most foci that a researcher might think of can be defined for the FIC. The focus only has to be expressed as a smooth function of the model parameters, cf. Chapter 2. One interesting choice for policy analysis might involve a differentiation between short- and long-run impulse response coefficients, cf. Chapter 9.1 of Lütkepohl (2005). We leave this for future research.

We will typically only present the impulse response functions based on a se- 


\section{CHAPTER 4}

lection of these model criteria to keep the figures uncluttered and well readable. The estimates based on the sAICa procedure, for example, usually lie very close to these of the AIC and sAICb methods by construction, and will for this reason often not be graphed. For joint confidence bands we apply the adjusted Bonferroni method described in Chapter 3 to pointwise confidence intervals obtained from the following bootstrap procedure.

Bootstrap Procedure Kilian (1998a) suggests reestimating the lag order in each bootstrap replication, and shows the asymptotic validity of this method which we adopt when strongly consistent model selection criteria are used. Furthermore it is shown that the validity of the bootstrapped confidence intervals in this setting is still given when using AIC. Let xIC stand for the chosen information criterion. Then the algorithm is as follows.

1. Choose $\underline{p}, \bar{p}$. Fit $\operatorname{VAR}(p)$ models, $\underline{p} \leq p \leq \bar{p}$, to the data $\left\{\boldsymbol{y}_{t}\right\}$,

$$
\boldsymbol{y}_{t}=\widehat{\boldsymbol{A}}_{1} \boldsymbol{y}_{t-1}+\widehat{\boldsymbol{A}}_{2} \boldsymbol{y}_{t-2}+\ldots+\widehat{\boldsymbol{A}}_{\widehat{p}} \boldsymbol{y}_{t-\widehat{p}}+\widehat{\boldsymbol{u}}_{t}
$$

Determine $\widehat{p}$ by minimizing the xIC.

2a. Generate bootstrap replications $\left\{\boldsymbol{y}_{t}^{*}\right\}$ based on the recursion

$$
\boldsymbol{y}_{t}^{*}=\widehat{\boldsymbol{A}}_{1} \boldsymbol{y}_{t-1}^{*}+\widehat{\boldsymbol{A}}_{2} \boldsymbol{y}_{t-2}^{*}+\ldots+\widehat{\boldsymbol{A}}_{\widehat{p}} \boldsymbol{y}_{t-\widehat{p}}^{*}+\boldsymbol{u}_{t}^{*}
$$

where the $\left\{\boldsymbol{u}_{t}^{*}\right\}_{t=1}^{T}$ are obtained as follows: Let $K$ be the number of variables. Draw randomly with replacement from the $\widehat{\boldsymbol{u}}_{t}$ multiplied by $\left(\frac{T}{T-K \bar{p}-1}\right)^{1 / 2}$ (cf. Davidson \& MacKinnon (2004)). Demean the set of $T$ draws.

For each bootstrap replication $\left\{\boldsymbol{y}_{t}^{*}\right\}$

2b. Fit $\operatorname{VAR}(p)$ models, $\underline{p} \leq p \leq \bar{p}$, to the series and determine $\widehat{p}^{*}$ by minimizing the xIC. (endogenous lag order bootstrap)

3. Calculate the impulse response coefficient $\widehat{\theta}^{*}\left(\widehat{\boldsymbol{A}}_{1}^{*}, \ldots, \widehat{\boldsymbol{A}}_{\widehat{p}^{*}}^{*}, \widehat{\boldsymbol{\Sigma}}^{*}\right)$.

This procedure yields a distribution of the estimates of the impulse response coefficient $\theta$. Pointwise confidence bands for a nominal coverage of $1-\alpha$ can then be obtained by reading off the $\alpha / 2$ and $1-\alpha / 2$ percentile interval endpoints of the set of bootstraped estimates $\widehat{\theta}^{*}$. Their standard deviation is the standard error of $\theta$, which is reported in Table 4.4 for some responses.

A variation of this method is the exogenous lag order algorithm: Leave out step $2 \mathrm{~b}$, and set $\widehat{p}^{*}=\widehat{p}$ instead.

Analogously to these bootstrap algorithms for model selection estimators we define counterparts for averaging estimators in Section 3.4. 


\subsection{ESTIMATES}

\subsection{Estimates}

This section details the specifications used in the three different empirical studies. The first paragraph containing Table 4.1 gives an overview of the different data sets and model specifications, which are subsequently laid out in detail for each of the studies separately, together with plots of the resulting impulse response functions. The discussion of the results takes place in Section 4.4.

Overview The specifications of all three data sets are comprehensively listed in Table 4.1. The three studies used slightly different time series. The quarterly data set of Stock and Watson (2001) includes all the years from 1960 until 2000, Christiano et al. (1999)'s quarterly time series lasts from 1965:Q3 until 1995:Q2, and Uhlig (2005) works with monthly data stretching from 1965 to 2003. The chosen lag order of four in the first two studies, and twelve in the last one hence all correspond to the time horizon of one year. But Ivanov and Kilian (2005) note that in general dynamcics for models with the same variables measured at different frequencies are different. ${ }^{2}$ And when comparing the different results we make the same observation as well, see below. Note however that Uhlig (2005) may be a special case, since the monthly data has been obtained by interpolation from lower frequency data. The interpolation method for acquiring monthly data used by Uhlig (2005) is described in Bernanke et al. (1997) and Bernanke and Mihov (1998). Temporal aggregation of time series is the opposite operation. Low frequency data for flow variables are aggregates from high frequency data. Silvestrini and Veredas (2008) survey the current state of the literature on temporal aggregation.

The chosen parametrization for ensuring identification of a structural monetary policy shock implies a contemporaneous effect of zero of certain variables. For Stock and Watson (2001) these are the inflation response to a shock in unemployment, and the responses of inflation and unemployment to a monetary policy shock. For the other data sets these are the responses of real GDP, the GDP price deflator and the commodity price index to an exogenous monetary policy shock.

Stock and Watson (2001) Stock and Watson (2001) employ a three variable quarterly data set consisting of the rate of price inflation ${ }^{3}$, the unemployment -, and the federal funds interest rate for the period 1960:Q1-2000:Q4, T = 160 observations. The order of the variables in the respective VAR corresponds to the order in which they are named in this section for all three data sets. A unit root in the federal funds rate cannot be rejected with a p-value of 0.38 , and the largest absolute value of the roots in the VAR(4) that they choose to estimate is 0.9714. But we do not impose a unit root for estimation for the reasons discussed in Section 4.2 above. We set $\bar{p}=12$ and report additional results for $\bar{p}=9$ in the

\footnotetext{
${ }^{2}$ Obviously, for VARMA models the parameters of the model for the low frequency observations are functions of the parameters of the model for the high frequency observations, also cf. Silvestrini and Veredas (2008).

${ }^{3}$ Stock and Watson (2001): "The inflation data are computed as $\pi_{t}=400 \ln \left(P_{t} / P_{t-1}\right)$, where $P_{t}$ is the chain-weighted GDP price index".
} 


\section{CHAPTER 4}

\begin{tabular}{l|ccc} 
& SW & CEE & Uhlig \\
\hline frequency & quarterly & quarterly & monthly \\
period & 1960:Q1 - 2000:Q4 & 1964:Q3 - 1995:Q2 & 1965:01 - 2003:12 \\
impulses & all & monetary & monetary \\
benchmark lag order & 4 & 4 & 12 \\
max horizon & 24 & 16 & 60 \\
\hline 1 & Price & Y & Y \\
2 & unemployment & Price & Price \\
3 & FF & Pcom & Pcom \\
4 & $*$ & FF & FF \\
5 & $*$ & NonbR & NonbR \\
6 & $*$ & TotR & TotR \\
7 & $*$ & M1 & $*$ \\
\hline
\end{tabular}

Table 4.1: Specifications of the different sVAR studies. Impulses describes which impulse responses are plotted: SW plot all nine impulse response functions, the other studies only show the responses to a structural monetary shock. The second part of the table lists the variables in the ordering used for modelling. FF stands for the federal funds rate. Data sets of SW: Stock and Watson (2001), CEE: Christiano et al. (1999), Uhlig: Uhlig (2005). * stands for "not applicable".

appendix. $\bar{p}=12$ is chosen as it is the largest integer smaller than $\sqrt{T}$ as suggested in Chapter 3.5. Figure 4.2 shows the estimated impulse response functions, Table 4.2 the weights of the averaging estimators. It shows that, as expected, the different averaging estimators divide the weights differently between the lags, and this translates into different estimated response functions in Figure 4.2.

Christiano et al. (1999) Christiano et al. (1999)'s structural VAR models seven variables: The log of real GDP (Y), the log of the implicit GDP deflator (Price), the smoothed change in an index of sensitive commodity prices (Pcom), the federal funds rate (FF), the log of nonborrowed reserves plus extended credit (NonbR), the log of total reserves (TotR), and the log of M1 (M1), all quarterly observed in the period 1964:Q3-1995:Q2 resulting in 124 observations. ${ }^{4}$ The authors choose four lags, but offer no motivation for this choice, that corresponds to a one year period and implies a largest absolute root of 0.990 of the estimated lag polynomial. We set $\bar{p}=11$ as it is the largest integer smaller than $\sqrt{T}$ as suggested in Chapter 3.5, and provide some results for $\bar{p}=9$ in the appendix, Chapter 4.A.1, where we find that the estimated impulse responses remain similar for the most part, with the exception of those based on AIC model selection and the FIC estimator. Table 4.3 shows the models chosen by AIC, the weights

\footnotetext{
${ }^{4}$ The start date written in their study is 1965:Q3, but that does not include the 4 presample observations that are necessary for the impulse responses implied by the VAR(4) to match those presented by the authors.
} 

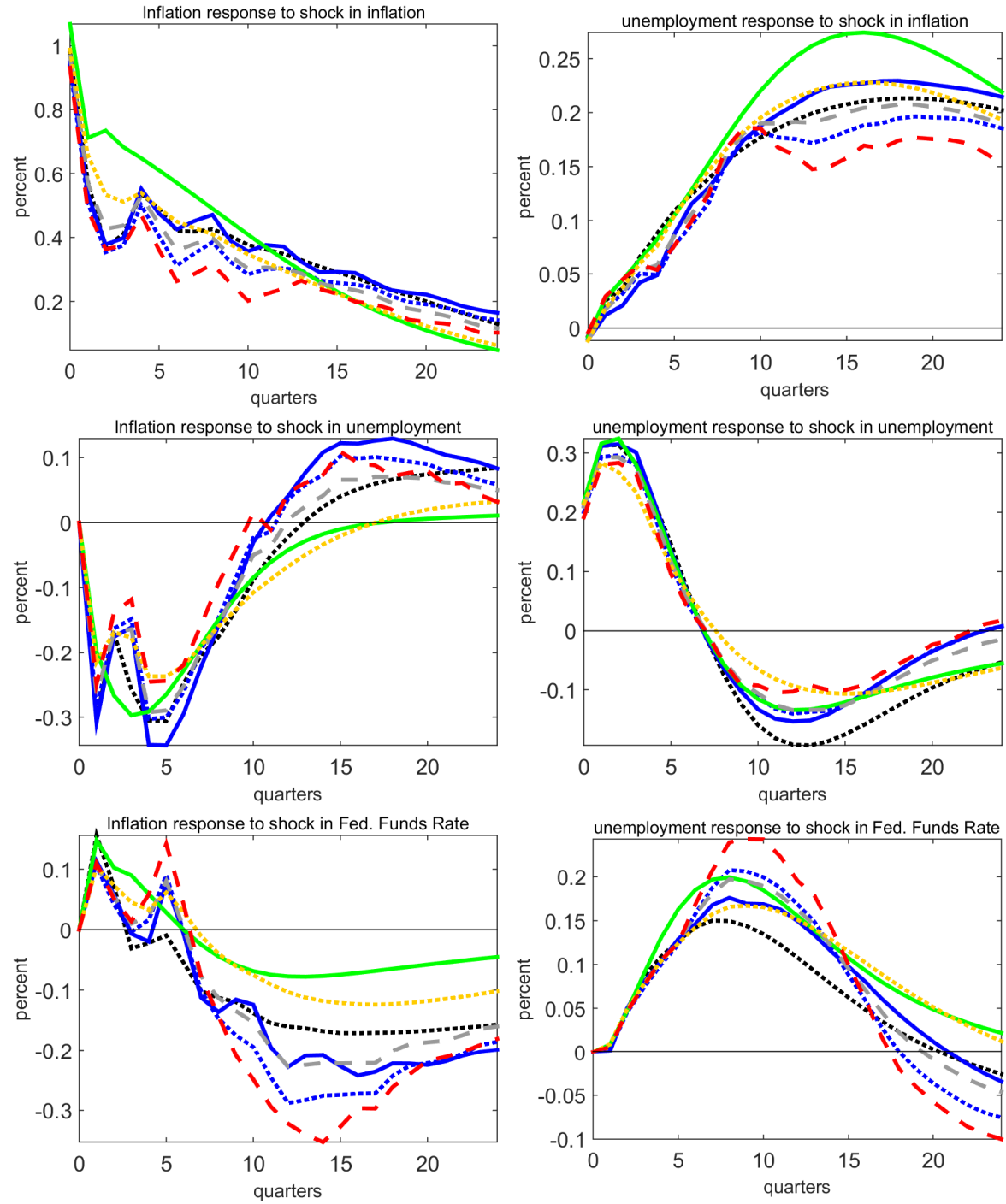

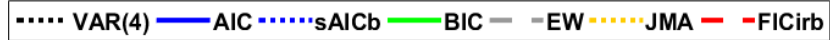

Figure 4.2: Stock and Watson (2001) data set IRs for $\bar{p}=12$.

Criteria line colors: Black: VAR(4). Blue: AIC (solid), sAICb (broken).

Green: BIC. Gray: Equal weights averaging. Orange: Jackknife model averaging. Red: FIC averaging. 

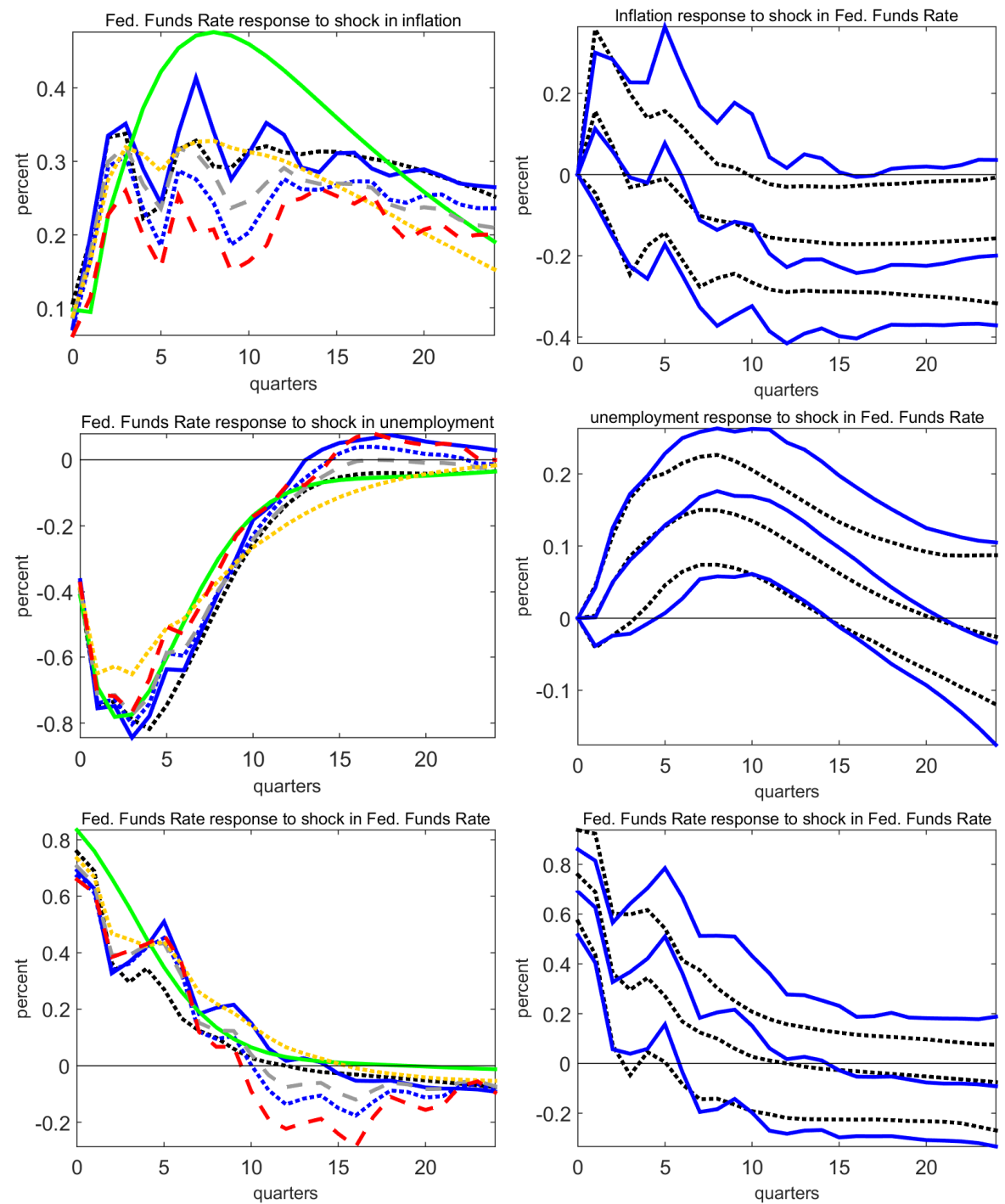

$$
\ldots . \cdot \text { VAR(4) - AIC } \cdots . \cdot \cdot \text {. }
$$

Figure 4.3: Stock and Watson (2001) data set IRs for $\bar{p}=12$. Left column: Point estimates. Right column: IR point estimates with $66 \%$ joint (adjusted Bonferroni) confidence bands, 5000 bootstrap replications. Criteria line colors: Black: VAR(4). Blue: AIC (solid), sAICb (broken). Green: BIC. Gray: Equal weights averaging. Orange: Jackknife model averaging. Red: FIC averaging. 


\begin{tabular}{l|ccc} 
lag & sAICb & FICirb & JMA \\
\hline 1 & 0.00 & 0 & 0.25 \\
2 & 0.02 & 0.19 & 0.26 \\
3 & 0.07 & 0.04 & 0.11 \\
4 & 0.07 & 0 & 0.03 \\
5 & 0.06 & 0 & 0 \\
6 & $\mathbf{0 . 1 3}$ & 0 & 0.26 \\
7 & 0.08 & 0 & 0 \\
8 & 0.09 & 0 & 0.05 \\
9 & 0.12 & 0 & 0 \\
10 & 0.12 & 0 & 0 \\
11 & 0.12 & 0.33 & 0.05 \\
12 & 0.10 & 0.44 & 0 \\
\hline
\end{tabular}

Table 4.2: Weights of model averaging methods sAICb, FICirb, jackknife model averaging for the Stock and Watson (2001) data set. The lag order selected by the AIC is marked in boldface.

assigned by sAICb, FICirb, and JMA. The BIC selects $p=1$ lags, AIC selects the largest model $p=\bar{p}$.

The impulse response estimates based on using the different methods are plotted in Figures 4.4 - 4.6. Point estimates are given in the left column, point estimates together with bootstrapped $95 \%$ confidence bands in the right columns. Table 4.3 tells us, that sAICb divides the weights among all models with higher weights on the larger models.

The confidence bands in Christiano et al. (1999) are obtained from a residual (Sieve) bootstrap with 500 replications (cf. their footnote 23). These are what is termed naive confidence bands by Lütkepohl et al. (2015). The bands are constructed by connecting pointwise confidence intervals, which typically results in bands that have lower effective coverage of the impulse response function than intended. This can be seen in the figures: Together with the naive bands we plotted the bands calculated by the adjusted Bonferroni method (cf. Chapter 3 and Lütkepohl et al. (2015)), which are wider. Also plotted are the point estimates and the adjusted Bonferroni confidence bands based on sAIC model averaging. Note that the confidence intervals for the $\operatorname{VAR}(4)$ implied impulse reponses are not directly comparable to those obtained from the other methods, because the endogenous lag order bootstrap allows to reestimate the lag order for every bootstrap iteration, while for the $\operatorname{VAR}(4)$ method a $\operatorname{VAR}(4)$ is estimated in every bootstrap iteration. But as mentioned above Christiano et al. (1999) did not specify which method or which model selection criterion they used to decide on their specification.

Uhlig (2005) Uhlig (2005) extends Bernanke and Mihov (1998)'s monthly (acquired through interpolation) data set to the period 1965:01 - 2003:12 yielding 468 

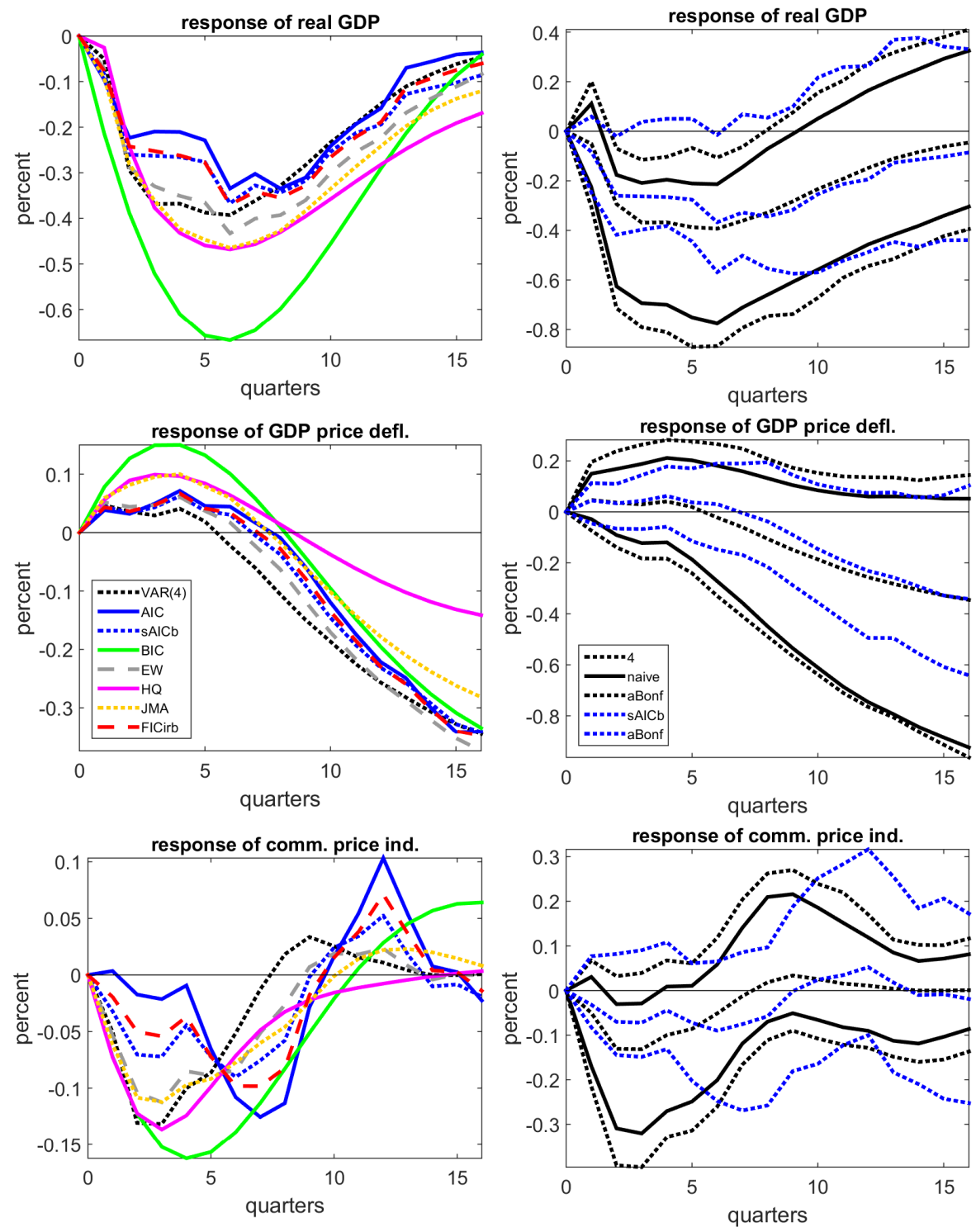

Figure 4.4: Christiano et al. (1999) data set IRs, responses to a contractionary monetary policy shock one standard deviation in size. Left column: point estimates. Right column: 95\% endogenous lag order bootstraped confidence intervals (naive and adjusted Bonferroni), 5000 bootstrap replications. Criteria line colors: Black: VAR(4). Blue: AIC (solid), sAICb (broken). Green: BIC. Gray: Equal weights averaging. Magenta: Hannan-Quinn selection. Orange: Jackknife model averaging. Red: FIC averaging. 

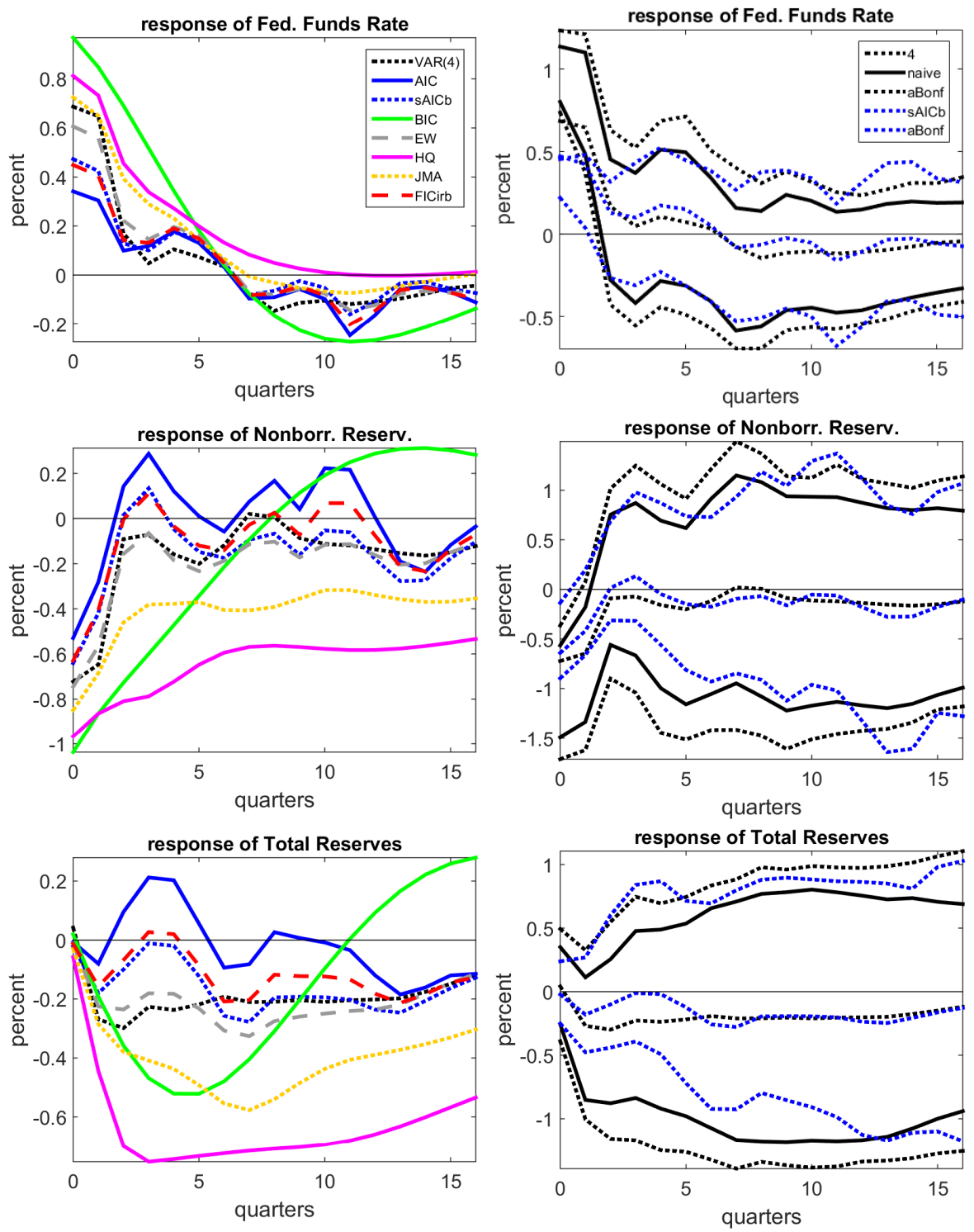

Figure 4.5: Christiano et al. (1999) data set IRs, responses to a contractionary monetary policy shock one standard deviation in size. Left column: point estimates. Right column: 95\% endogenous lag order bootstraped confidence intervals (naive and adjusted Bonferroni), 5000 bootstrap replications. Criteria line colors: Black: VAR(4). Blue: AIC (solid), sAICb (broken). Green: BIC. Gray: Equal weights averaging. Magenta: Hannan-Quinn selection. Orange: Jackknife model averaging.

Red: FIC averaging. 


\section{CHAPTER 4}

\begin{tabular}{l|ccc} 
lag & sAICb & FICirb & JMA \\
\hline 1 & 0.02 & 0 & 0.31 \\
2 & 0.04 & 0.08 & 0.45 \\
3 & 0.04 & 0.09 & 0 \\
4 & 0.04 & 0 & 0.06 \\
5 & 0.04 & 0 & 0 \\
6 & 0.04 & 0.11 & 0 \\
7 & 0.05 & 0 & 0 \\
8 & 0.06 & 0 & 0 \\
9 & 0.14 & 0.09 & 0.12 \\
10 & 0.16 & 0 & 0.06 \\
11 & $\mathbf{0 . 3 7}$ & 0.63 & 0 \\
\hline
\end{tabular}

Table 4.3: Weights of model averaging methods sAICb, FICirb, jackknife model averaging for the Christiano et al. (1999) data set. The lag order selected by the AIC is marked in boldface.

observations. It contains six variables: real GDP, GDP price deflator, a commodity price index, the federal funds rate, nonborrowed reserves, and total reserves. A Dickey Fuller test fails to reject the Null of a unit root in the federal funds rate with a p-value of 0.3 . The largest root of the estimated $\operatorname{VAR}(12)$ is 1.0003 . Uhlig (2005) uses a VAR(12) without constant or trend to model the log levels except for the federal funds rate, which is taken as such instead of in logarithms. The decision on twelve lags is based on LR tests starting with 15 . We use this as $\bar{p}=15$. When applying model selection criteria to the data set AIC would select $\widehat{p}=3$, and BIC $\widehat{p}=2$. However these small models do not capture the dynamics completely: Ljung-Box tests for the individual series of residuals indicate significant (at $5 \%$ significance level) autocorrelation of order eight and higher (mostly for the federal funds rate). When estimating models with nine or more lags this is not the case, so we set $p=9$. With this restricted set of models, $9=p \leq p \leq \bar{p}=15$, AIC, BIC and also $\overline{\mathrm{H}} \mathrm{Q}$ end up selecting the same model of lag order nine. And also the sBIC and JMA averaging methods put all weight on that model. The weights of the sAICb estimator are $w=(0.25,0.19,0.15,0.14,0.11,0.10,0.06)$. The corresponding IRs of a one standard deviation shock to the federal funds rate are graphed in Figure 4.7 and are discussed in Section 4.4 below. As a robustness check we also set $\bar{p}=21$, the largest integer smaller than $\sqrt{T}$. The resulting shapes of the impulse response functions were similar to those for $\bar{p}=15$ for all methods except for the equal weights and the FIC methods. The graphs are available from the author upon request.

Uhlig (2005) plots medians along with their 68\% confidence bands obtained from a Bayesian procedure. Similarly we plot the bootstrapped $68 \%$ adjusted Bonferroni confidence bands for the estimates based on the sAICb method and the VAR(12) in Figure 4.8 and for the AIC-based estimates in Figure 4.9. 


\subsection{DISCUSSION}
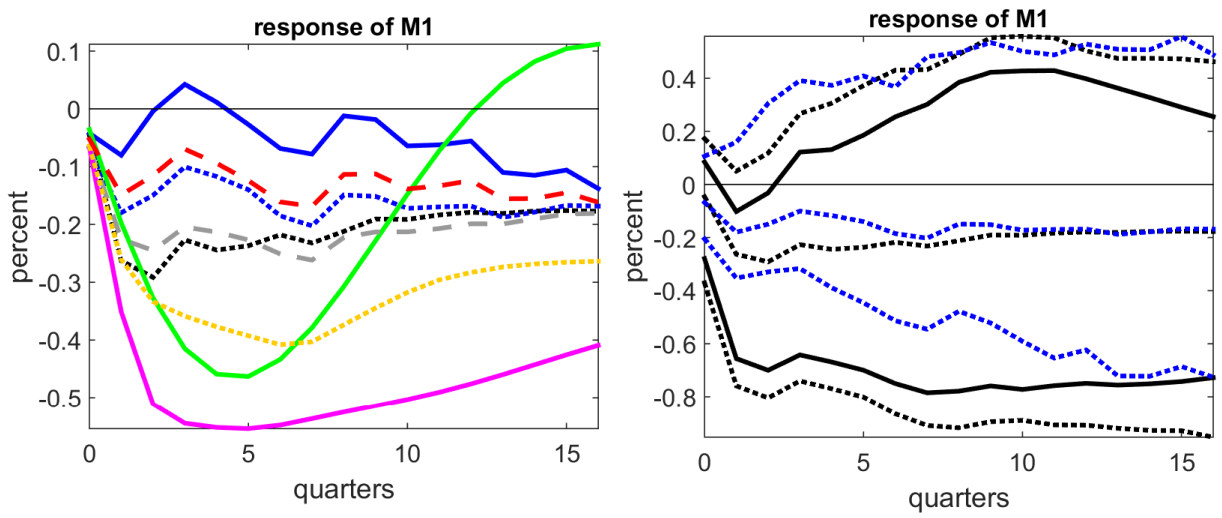

Figure 4.6: Christiano et al. (1999) data set IRs, responses to a contractionary monetary policy shock one standard deviation in size. Left graph: point estimates. Criteria line colors: Black: VAR(4). Blue: AIC (solid), sAICb (broken). Green: BIC. Gray: Equal weights averaging. Magenta: Hannan-Quinn selection. Orange: Jackknife model averaging. Red: FIC averaging. Right graph: 95\% endogenous lag order bootstraped confidence intervals, 5000 bootstrap replications. Line colors: Black broken: VAR(4) estimate with adjusted Bonferroni confidence bands. Black solid: VAR(4) naive confidence band. Blue: sAICb estimate with adjusted Bonferroni confidence bands.

\subsection{Discussion}

Comparison of Benchmark Estimates We first discuss the results based on the authors' chosen lag order of one year corresponding to a VAR(12) for the Uhlig (2005) data set, and a VAR(4) for the other two data sets. While the three data sets are not the same, they have been chosen for our analysis because they share some common variables, some of which have similarly shaped dynamic responses across all studies, and the same research question, namely what the effects of an exogenous, contractionary monetary policy shock one standard deviation in size are. This corresponds to a shock in the third structural innovation in the Stock and Watson (2001) model, and in the fourth in the models of Christiano et al. (1999) and Uhlig (2005), cf. Table 4.1. If not noted differently, all the analyses of this chapter refer to such a shock.

The impulse response functions that are especially similar across all studies are those of the federal funds rate. Also, for the response of the general inflation measure, for example, we can observe that it is positive in the beginning, and turns negative in the long run for all three studies. Let us look at the response of the federal funds rate in Figures 4.3 and following in more detail. The federal fund rate's response function rises contemporaneously to a value between $0.5 \%$ and $0.75 \%$ and becomes slightly negative over the course of four to five years in 

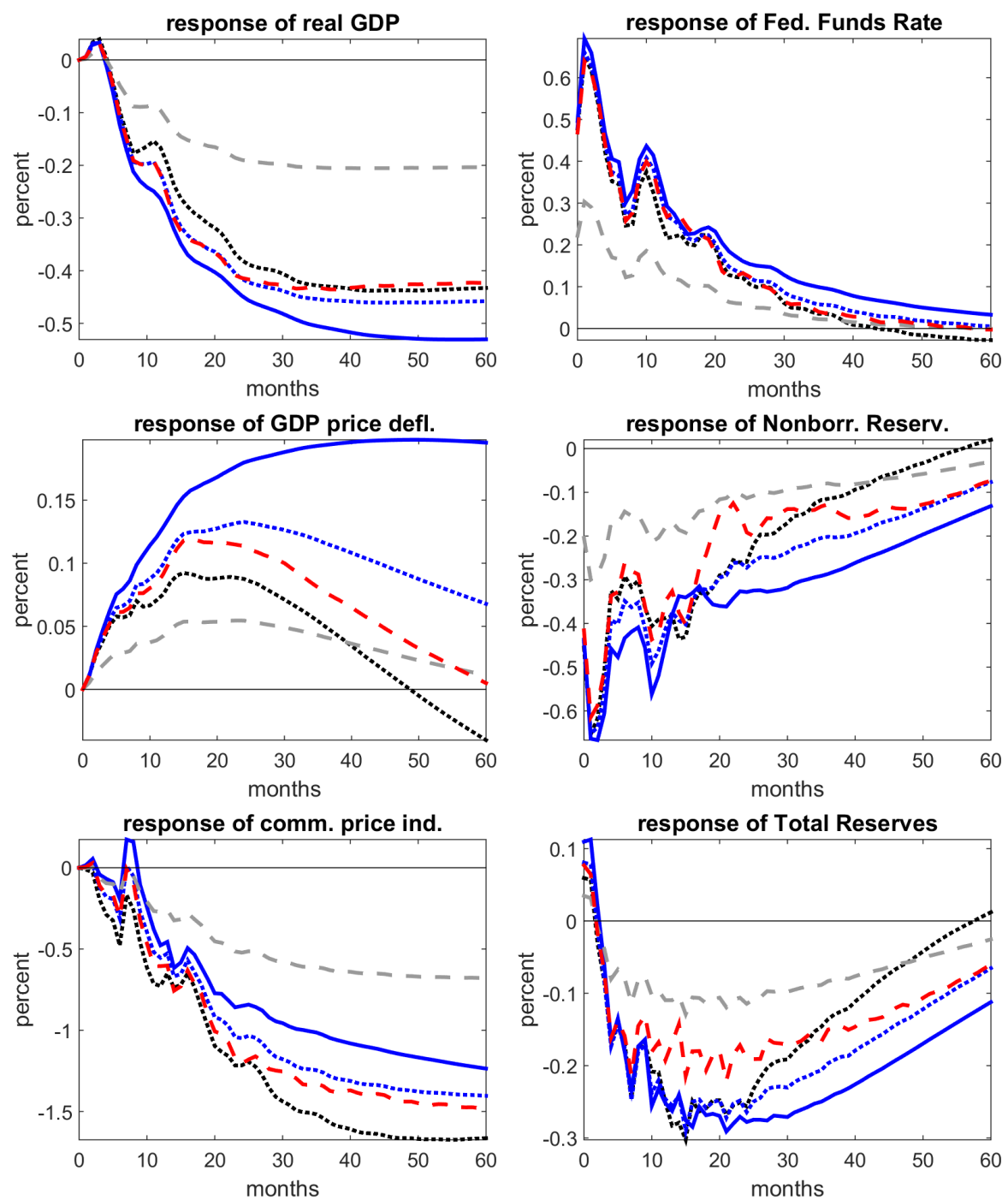

......VAR(12) -AIC ......sAICb - -EW - -FICirb

Figure 4.7: Uhlig (2005) data set IRs, responses to a contractionary monetary policy shock one standard deviation in size. AIC, BIC, HQ, sBIC, and JMA all select or put all weight on a $\operatorname{VAR}(9)$ and hence their impulse response functions coincide. 

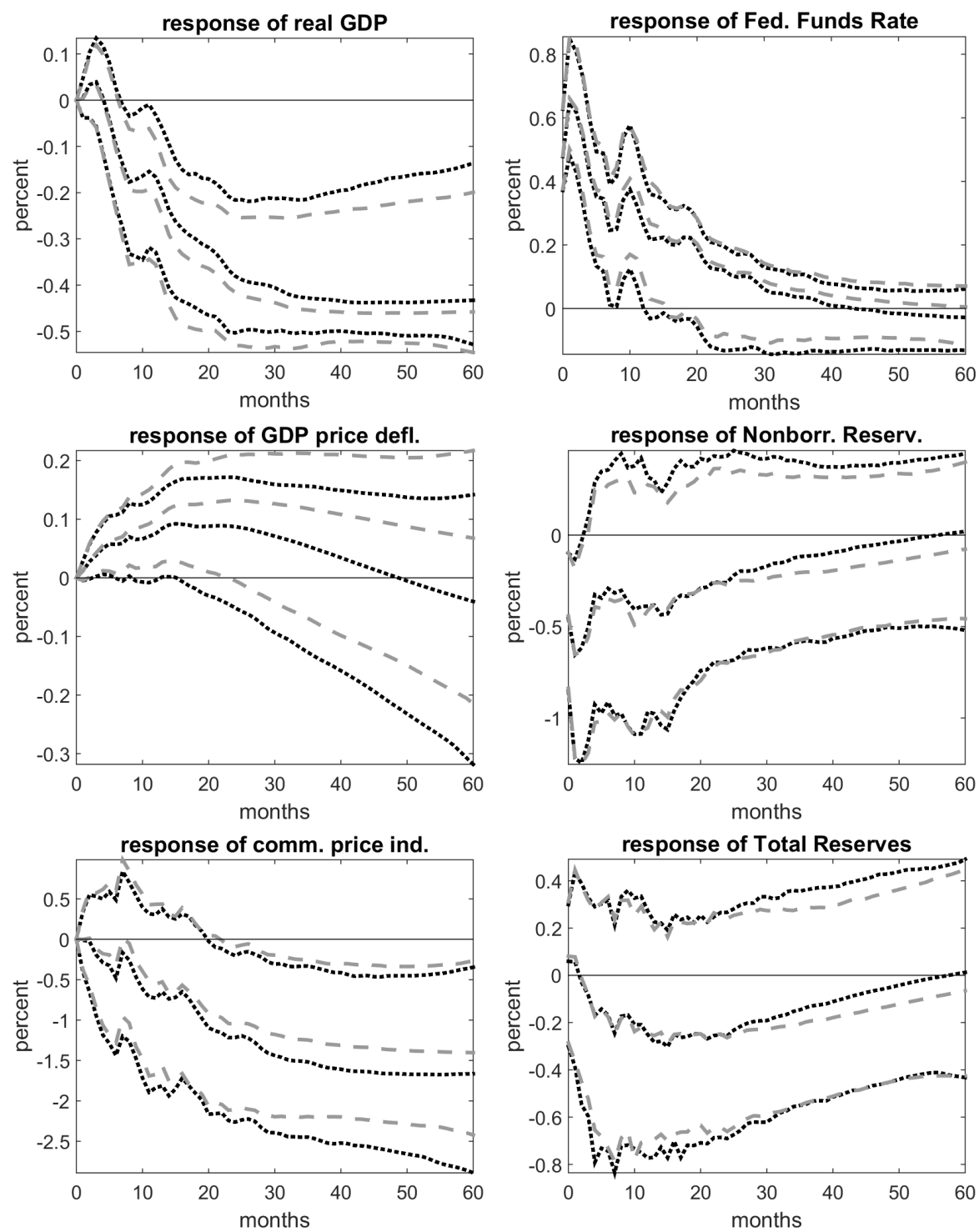

-..-VAR(12) - - sAICb

Figure 4.8: Uhlig (2005) data set IR estimates based on $\mathrm{sAICb}$ and the $\operatorname{VAR}(12)$ with $68 \%$ adjusted Bonferroni bootstrapped confidence bands (exogenous lag order bootstrap) from 5000 bootstrap replications. 

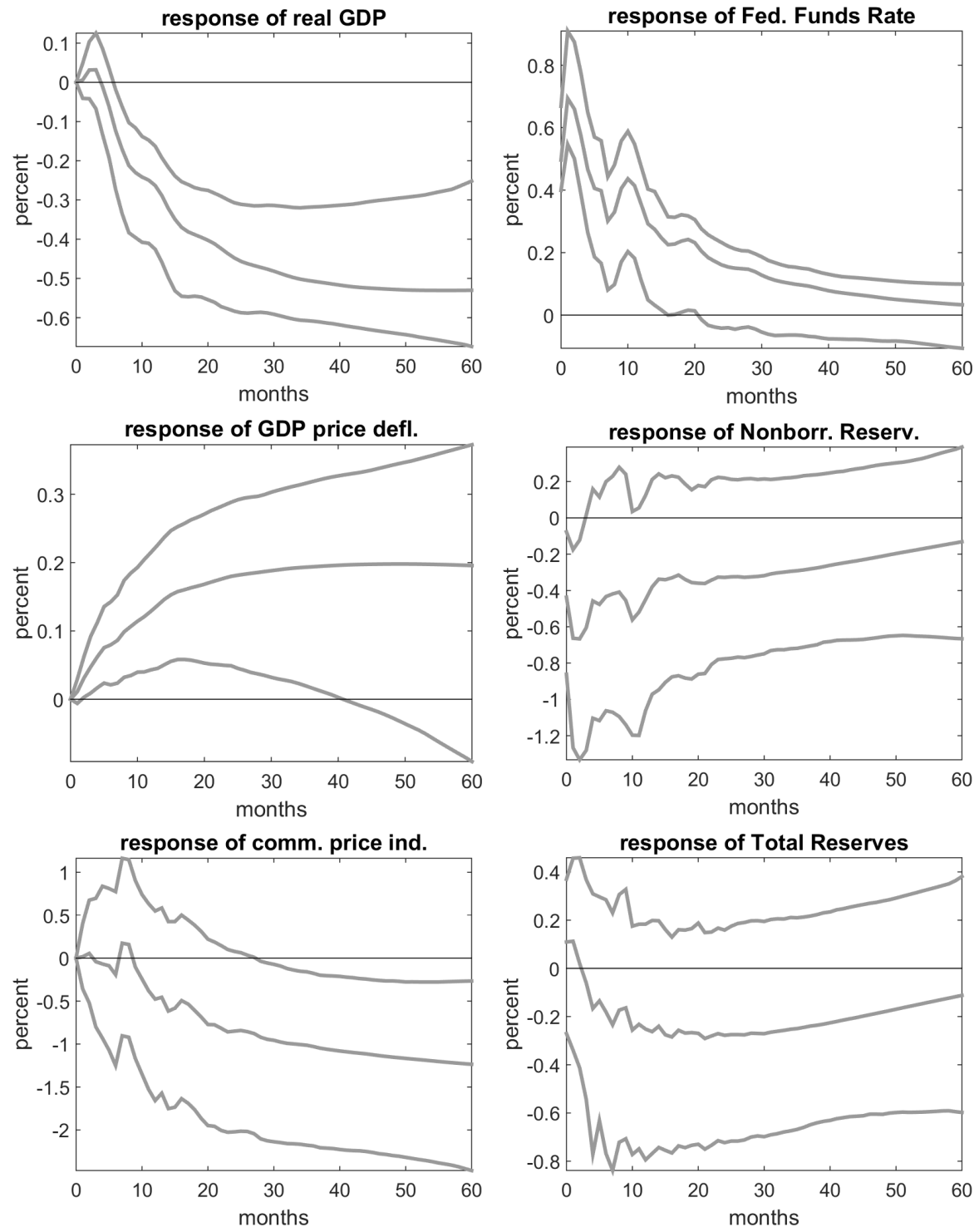

Figure 4.9: Uhlig (2005) data set IRs of the AIC-based estimator with $68 \%$ adjusted Bonferroni bootstrapped confidence bands (exogenous lag order bootstrap). 


\subsection{DISCUSSION}

all cases. The monthly data set shows an increase in the federal funds rate from the contemporaneous effect to the first month, indicating that the full effect of a shock only occurs after a few months. This spike gets evened out by using the quarterly frequency of the other two data sets. The second hump at around 1 year after the shock is common to all three studies.

The response of the general inflation measure is restricted to zero initially by our choice of parametrization, but then shows the response known as the price puzzle, cf. Uhlig (2005) and Sims (1992), rising at first before turning negative in the long run. Uhlig (2005) points out that the remedy suggested by Eichenbaum (1992) of including commodity prices does not resolve the price puzzle. From that point of view one might argue that the small set of variables used by Stock and Watson (2001) is enough to capture the dynamics of prices and interest rates, but on the other hand the confidence bands of the inflation response still do contain $0 \%$ for the beginning quarters in the Stock and Watson (2001) data set, cf. Figure 4.3 , so there the evidence for the existence of the price puzzle is weaker than for the Uhlig (2005) data. This is also the case for the Christiano et al. (1999) data. For the higher frequency data of Uhlig (2005) the response of the GDP price deflator does not become as negative in the long run as for the other data sets, and it remains in the positive region longer, cf. Figure 4.7.

This type of delayed reaction in the responses of the Uhlig (2005) data can also be observed for the other variables. While the response of real GDP remains at around $-0.4 \%$ even after five years, Christiano et al. (1999) describe a hump shaped response with the lowest point after around one and a half years and a return to almost original levels after four years, cf. Figure 4.4. One reason for the differing dynamics might be that the data sets end in different years, since, in general, estimates of underlying model parameters are not constant through time, which can be due to measurement errors or actual changes in the DGP, cf. e.g. Chapter 9 of Juselius (2006). Another reason for differing dynamics could be the different frequency of Uhlig (2005)'s time series compared to the other two, as discussed in Section 4.3 above.

The response of the commodity price index reaches values of around $-1.5 \%$ in both models where it is a part of the analysis, cf. Figures 4.4 and 4.7. However, from a little more than two years on the response in Uhlig (2005)'s model stays almost flat while Christiano et al. (1999) describe an increase back to above 0 with the response dying out after about three years. In contrast to the response of real GDP and that of the commodity price index the responses of both bank reserves measures appear more dynamic for the estimates based on the Uhlig (2005) data set. After initial drops they move back up to reach slightly positive values after five years while the responses estimated from Christiano et al. (1999)'s data almost stagnate after about half a year. The main conclusion that Christiano et al. (1999) draw from their model about the reaction of bank reserves also holds for Uhlig (2005)'s estimates:

"First, there is a persistent rise in the federal funds rate and a persistent drop in nonborrowed reserves. This finding is consistent with the presence of a strong liquidity effect. Second, the fall in total reserves is negligible initially [...] So according to this policy shock 


\section{CHAPTER 4}

measure, the Fed insulates total reserves in the short run from the full impact of a contraction in nonborrowed reserves by increasing borrowed reserves."

Out of the three considered studies the unemployment rate is only part of the Stock and Watson (2001) data set, but the studies of Bernanke and Blinder (1992), and Christiano et al. (1996) come to the same observation as can be made here, that it rises after a delay of about two quarters (Christiano et al. (1999), p. 26). The money supply measure M1 is only modelled by Christiano et al. (1999). Belongia and Ireland (2015) illustrate the benefits of including money aggregates in VARs evaluating monetary policy. The response of M1 to the monetary shock is negative and very similar to that of total reserves as pointed out in their study, cf. Figures 4.5 and 4.6 .

One interesting question raised by our choice of data sets is what the effects of measuring the variables at different frequencies are. Is it beneficial for estimator precision to use monthly instead of quarterly data? Note that while Bernanke and Mihov (1998) use a monthly as well as a biweekly data set and compare the estimates of both, they do not explicitly report standard errors for the estimated impulse responses, but only for some policy parameters. Since the number of variables included in our three different data sets differ, and they do not cover the same periods, we cannot draw definite conclusions, but beginning with discussing the precision of the federal funds rate response estimate should give us an idea what the answer might be, since this variable behaves similarly across data sets. As mentioned above we are concerned with the responses to a contractionary monetary policy shock one standard deviation in size. Table 4.4 reports the average widths of the confidence bands of some relevant impulse response functions, and standard errors of impulses, estimated by the benchmark VAR models ${ }^{5}$. See Section 4.2 for the technical details of the calculation methods. Similarly to Lütkepohl et al. (2015) we calculate the average width as the sum of the widths of the individual pointwise confidence intervals divided by the number of periods, which we set to correspond to four years, so $48+1$ periods for the monthly, and $16+1$ periods for the quarterly data sets. The bootstrapped standard errors of the impulse response at the horizon corresponding to four years are also given. The table entries corresponding to the federal funds rate's response are marked in bold face. This variable is ordered third in the Stock and Watson (2001) data set, and fourth in the other two. Those variables whose impulse response functions cannot be meaningfully compared with those of the other data sets, for example because they cover different ranges in the Christiano et al. (1999) and Uhlig (2005) analyses, are not shown. Note, for example, that the data sets of Christiano et al. (1999) and Uhlig (2005) both use the same types of variables, and ordering of the variables for the most part. But the money supply variable M1 is additionally included in the former, and the impulse responses of the commodity price index move over different ranges for the two different data sets: between $-0.15 \%$ and $0.1 \%$ in the former, and between $-1.7 \%$ and $0.2 \%$ in the latter. This finding might

\footnotetext{
${ }^{5}$ A VAR(4) for Stock and Watson (2001) and Christiano et al. (1999), and a VAR(12) for Uhlig (2005).
} 


\subsection{DISCUSSION}

possibly be explained by a difference in the concrete choice of index since Christiano et al. (1999) explicitly state that they employ the smoothed change in an index of sensitive commodity prices.

\begin{tabular}{l|ccc|ccc|ccc} 
& \multicolumn{7}{|c|}{ average widths } & \multicolumn{3}{c}{ standard errors } \\
& & naive & \multicolumn{2}{c}{ adj. Bonferroni } & & & \\
variable & SW & CEE & Uhlig & SW & CEE & Uhlig & SW & CEE & Uhlig \\
\hline 1 & $*$ & 0.26 & 0.18 & $*$ & 0.49 & 0.28 & $*$ & 0.16 & 0.13 \\
3 & $\mathbf{0 . 2 5}$ & $*$ & $*$ & $\mathbf{0 . 4 4}$ & $*$ & $*$ & $\mathbf{0 . 1 3}$ & $*$ & $*$ \\
4 & $*$ & $\mathbf{0 . 3 3}$ & $\mathbf{0 . 1 7}$ & $*$ & $\mathbf{0 . 6 4}$ & $\mathbf{0 . 3 1}$ & $*$ & $\mathbf{0 . 1 3}$ & $\mathbf{0 . 0 7}$ \\
5 & $*$ & 0.88 & 0.65 & $*$ & 1.58 & 1.11 & $*$ & 0.44 & 0.38 \\
6 & $*$ & 0.78 & 0.57 & $*$ & 1.31 & 0.94 & $*$ & 0.41 & 0.36 \\
\hline
\end{tabular}

Table 4.4: Average (over four years) widths of the $68 \%$ naive and adjusted Bonferroni confidence bands around the response to a monetary policy shock, and bootstrapped standard errors of the same response at horizon four years, estimated by the benchmark models. The average width is calculated as the sum of the widths of the intervals divided by the number of periods. The number in bold face marks the federal funds rate variable, which shows the smallest average width for the monthly data set. The other variable names are listed in Table 4.1. * stands for "not applicable". Data sets of SW: Stock and Watson (2001), CEE: Christiano et al. (1999), Uhlig: Uhlig (2005).

Table 4.4 shows that, indeed, the federal funds rate's response is estimated with highest precision with the monthly data set, and also for real GDP, and nonborrowed and total reserves the confidence bands are more narrow, and the standard errors smaller, for the monthly data, on average, than for the quarterly data. Including more variables in a structural VAR analysis, on the other hand, does not seem to improve precision based on these numbers, as the Christiano et al. (1999) study uses the highest number of variables among the three studies, namely seven. These observations follow equally from any of the three panels of the table, so from the widths of the naive and the adjusted Bonferroni confidence bands, as well as from the sizes of the standard errors. Generally, the size of standard errors is taken to represent (inverse) precision, while smaller confidence bands might also be due to lower effective coverage, a measure of accuracy. The study of Lütkepohl et al. (2015) considers the widths of the confidence bands calculated by several different methods as one criterion to make recommendations on the ranking of these methods.

Comparison of Model Selection and Averaging Methods We now discuss the differences in the estimates implied by using different model selection and model averaging methods.

It is interesting to note that the suggestions made by Ivanov and Kilian (2005) 


\section{CHAPTER 4}

yield estimates that are, for some impulse responses, farthest away from those given by the specifications of the authors of the studies we consider. Ivanov and Kilian (2005)'s suggestion is to use the model with the lowest HQ score for quarterly data. Consider the Christiano et al. (1999) data, for example. For the later horizons the estimated response of the DGP deflator, and the estimated responses of bank reserves and M1 are further away from the responses implied by Christiano et al. (1999)'s choice of lag order than those for the other methods. This is also the case for the responses of the GDP deflator and for the reserve responses at later horizons implied by AIC model selection for the Uhlig (2005) data set. And for monthly data Ivanov and Kilian (2005) suggest using AIC. There is one qualification to these suggestions, namely that the quarterly data should have at least 120 observations, which makes Christiano et al. (1999) a borderline case with 124 observations.

The BIC tends to select even smaller models (models with fewer lags) than HQ, and we see that the responses based on BIC are also often far away from those of the other methods, which, compared to the BIC and HQ, describe more or less similar shapes, cf. the response of nonborrowed reserves in Figure 4.5. This is not the case for the Uhlig (2005) responses where AIC, BIC and HQ select the same lag order. Kilian (2001) and Ivanov and Kilian (2005) advocate against using the BIC for lag order selection in the context of estimating impulse response functions, which seems a sensible suggestion based on our observation.

The impulse response functions typically become more ragged ${ }^{6}$ with increasing model size/lag order. This is shown in Figure 4.10 in the appendix, where it is also discussed that this behavior is not unique to structural VAR analyses, but that it already shows for stationary autoregressive settings. It goes in line with the increase in estimator volatility for increasing model size ${ }^{7}$, and the algebra of the impulse response calculation where each additional horizon means adding another estimated coefficient matrix as long as the horizon is smaller than the posited lag order. In the quarterly data sets AIC selects models with more than the four lags of the benmark model. These impulse responses are consequently more ragged, cf. e.g. the response of nonborrowed reserves in Figure 4.5, a behaviour that may be undesired for explaining macroeconomic reactions. We note that the AIC smoothed averaging method with $\kappa=d^{2}$, sAICb, literally yields smoother impulse response functions than its selection counterpart. These lie between the response functions of the benchmark model and the AIC-based model most of the time, cf. for example Figure 4.2. Next, we compare the precision of both methods. To quantify precision we use the bootstrapped confidence bands for model averaging methods, that were introduced in Chapter 3. Table 4.5 shows the average widths of the pointwise naive confidence intervals for the AIC and the sAICb method. The impulse responses are the responses to a monetary policy shock. The average is taken over all horizons plotted in the figures ${ }^{8}$. We see that the average width of the sAICb model averaging based method is always smaller than that of the AIC

\footnotetext{
${ }^{6}$ Ragged describing the opposite of monotonous. So a response that has more or higher spikes than another one would be termed more ragged.

${ }^{7}$ This in turn may be partly due to a reduction in the number of degress of freedom.

${ }^{8}$ So 24 quarters for Stock and Watson (2001), 16 for Christiano et al. (1999), and 60 months for Uhlig (2005).
} 


\subsection{DISCUSSION}

model selection based method. In summary the sAICb model averaging method yields smoother estimates with higher precision than the AIC model selection method. Together with the theoretical arguments of Chapter 3 this observation strengthens the case for using the model averaging versions of the AIC rather than doing AIC model selection.

\begin{tabular}{l|cc|cc|cc} 
& \multicolumn{2}{|c|}{ SW } & \multicolumn{2}{c|}{ CEE } & \multicolumn{2}{c}{ Uhlig } \\
variable & AIC & sAICb & AIC & sAICb & AIC & sAICb \\
\hline 1 & 0.25 & 0.23 & 0.21 & 0.20 & 0.20 & 0.18 \\
2 & 0.12 & 0.11 & 0.15 & 0.15 & 0.21 & 0.19 \\
3 & 0.32 & 0.30 & 0.11 & 0.11 & 1.38 & 1.30 \\
4 & $*$ & $*$ & 0.26 & 0.25 & 0.15 & 0.14 \\
5 & $*$ & $*$ & 0.63 & 0.62 & 0.63 & 0.60 \\
6 & $*$ & $*$ & 0.56 & 0.55 & 0.57 & 0.54 \\
7 & $*$ & $*$ & 0.33 & 0.33 & $*$ & $*$ \\
\hline
\end{tabular}

Table 4.5: Average widths of the pointwise naive $68 \%$ confidence intervals around the response to a monetary policy shock in the models with lag length chosen by the AIC selection criterion, model weights calculated by $\mathrm{sAICb}$, respectively. The averages are taken over the respective numbers of periods plotted in Figures 4.2 to 4.7. * stands for "not applicable". Data sets of SW: Stock and Watson (2001), CEE: Christiano et al. (1999), Uhlig: Uhlig (2005).

Turning to the other model averaging methods it is interesting to see that the FIC method and the sAIC method yield very similar estimates. This is especially the case for the quarterly data sets. The objectives of both approaches are actually quite different, cf. Hansen (2005). While AIC was developed with the idea of picking out the correct specification of the underlying model, the FIC is explicitly designed to optimize the estimate of a certain focus parameter. However, the specific methods applied here are at less extreme ends of these two philosophies, since as focus of the FICirb estimator we defined all the impulse response functions. Note that this also includes those not plotted, for example the response of real GDP to a shock in real GDP. With this more general focus than for example defining just the impulse response coefficient at some particular horizon as the focus parameter, the FIC becomes closer to a model selection criterion that aims at a global model fit. Claeskens and Hjort (2003) explicitly discuss the relationship between AIC and FIC in their Section 5.6 and show that minimizing the estimated average mean squared error of the log likelihood corresponds to using the AIC criterion. Also we compare the sAICb method with the FICirb method, which are both model averaging methods.

On the one hand it is interesting to note that the ad hoc choice for modelling a VAR(4) corresponding to 1 year in the quarterly data sets is validated, one could say by the fact that by applying likelihood ratio tests Uhlig (2005) also settles on a model that conditions on a 1 year period. But on the other hand Ivanov 


\section{CHAPTER 4}

and Kilian (2005) argue that data sets of different sampling frequency should be treated differently, and a qualitatively different behaviour between the quarterly data sets and the monthly data set is also described above for the responses of the GDP price deflator. Now, concentrating attention on the quarterly data sets of Christiano et al. (1999) and Stock and Watson (2001) the equal weights averaging estimates actually lie very close to the benchmark estimates, cf. Figures 4.2 to 4.6. For the monthly data set of Uhlig (2005) this is not the case. In terms of the robustness checks on the authors' results alluded to in the introduction this means the following for the quarterly data sets. Even though the authors did not arrive at their choice for conditioning on a 1 year period with formal testing, their choice can be argued to represent a nondiscriminatory average of the whole model set's estimates. So their choice of modelling a VAR(4) can actually be interpreted as taking an uninformed average over the whole model set. Stock and Watson (2004) found that in forecast combinations simple combination methods (they consider a mean forecast giving equal weight to each of the forecasts in the panel, among others) perform well in terms of forecasting error.

Above we already discussed that the BIC and HQ model selection criteria tend to lead to estimates that are rather different from those of the other methods. But these criteria also tend to select models that might be too small to capture the dynamics of the DGP. For the rest of the plotted criteria the general shapes of the impulse responses, and hence the conclusions that a researcher would draw from these, are similar. This holds especially for the methods of choosing a $\operatorname{VAR}(4)$, using AIC, sAICb, EW or FICirb for the quarterly data sets, as can be seen in the response of the federal funds rate to a shock in the federal funds rate in Figure 4.3, for example. At 24 quarters the response is -0.1 no matter which model selection or averaging method is used. This means that the results of the sVAR analyses are relatively robust to the choice of method for deciding on a lag order. This does not seem to be the case as much for the monthly data set. The response of the GDP price deflator after 60 months is estimated to be negative when a $\operatorname{VAR}(12)$ is used, but positive at 0.2 when AIC is used for model selection, cf. Figure 4.7. But also note that the $68 \%$ confidence bands around the AIC based point estimate still contain 0, cf. Figure 4.9 .

\subsection{Conclusion}

The three data sets that we consider have many similarities, but also differ across several dimensions, e.g. number of variables and sampling period. Therefore we carefully choose a few variables for measuring the precision of their estimators. We find that in terms of precision it is beneficial to sample data at higher frequency (monthly instead of quarterly), but that the extension of a model's conditioning set by including more variables does not increase precision. This is interesting because it is an argument in the same direction as Uhlig (2005) makes, who states that also in terms of remedying the price puzzle, including more variables, in this case a commodity price index, is not helpful. This prize puzzle, that is observing a positive hump in the DGP deflator's response in the beginning periods, is one 
of the two patterns, that we observe across all specifications in a more or less pronounced fashion. The other robust observation concerns the dynamics of the federal funds rate, whose response returns to slightly negative levels in the long-run for all specifications. These two observations are robust to changes in the variables used in the model, the sampling period, the maximum lag order $\bar{p}$ and even the model selection or model averaging criterion. However, the length of time for the response of inflation to remain in the positive region differs across data sets, and so does its long-run impact. This brings us to those macroeconomic variables whose responses are less robust to modelling changes. Based on our results we would be less confident in making a general claim about the long-run effect on real GDP, a very important variable to policy makers. Its response to a monetary shock is not significantly different from zero for the Christiano et al. (1999) setting, but clearly negative with the Uhlig (2005) estimates. For the general inflation measure we also observe different responses for different specifications, and, additionally, the choice of model selection method shows to have a large effect on inflation: While after four years it is estimated to be negative for the quarterly data sets, cf. Figures 4.2 and 4.4 , it could be as high as $0.2 \%$, depending on the method of selecting a lag order, for the Uhlig (2005) data, cf. Figure 4.7. Different estimated responses for different data sets might be due to the differences in sampling periods or also in sampling frequency. The survey of Silvestrini and Veredas (2008) is a good starting point when discussing how time series from the same DGP sampled at different frequencies relate to each other. It would be interesting, in future research, to single out which differences in the responses are caused by which differences in the data sets, by e.g. shortening the sampling periods to make them equal across all three studies.

From the different model selection and model averaging methods, that we apply, we draw the following conclusions for the structural VAR analysis of empirical data. We cannot agree with the suggestion of Ivanov and Kilian (2005) of using HQ model selection for quarterly data sets. The application of our model averaging methods mostly delivers results similar to those of the model selection methods that are already routinely used by researchers. This is an expected finding in view of Chapters 2 and 3. These methods seem to be an interesting and useful addition to the macroeconomist's toolbox. It is especially interesting to note that for quarterly data the equal weights estimator can be considered an alternative to the benchmark estimates, that are based on a lag order corresponding to one year. The two methods that have, to our knowledge, not been applied in this context before, are sAICb and FICirb. While AIC is a well established criterion for model selection in policy analysis, the impulse response estimates implied by AIC selection show sharp peaks in some of the graphs above. Here its smooth model averaging versions, like the sAICb, offer an alternative with more intuitive, smoother, and more precise estimates. Interestingly, the specific FIC averaging method that we use delivers estimates rather similar to the sAICb method. FIC methods are especially interesting because of their underlying theory that allows them to be specifically defined for estimating the impulse response functions, and we believe that our results are an encouragement to applied researchers to consider the use of these for sVAR analyses. 


\section{CHAPTER 4}

From a theoretical perspective there remain challenges for two groups of estimators that are being applied in this chapter. In Chapter 2 we show that the impossibility of consistently estimating the misspecified parameters causes the feasible FIC-based estimators to have lower accuracy than the respective infeasible estimators. And Leeb and Pötscher (2005) show in a series of papers that the distributions of post-model-selection estimators cannot be estimated consistently uniformly over the parameter space. In light of this finding the authors implicitly advise to use estimators like, for example, the bootstrapped confidence bands that we calculate, with caution. Both findings are similar insofar as the theory for both is based on models where some of the parameters are local to zero. From an applied perspective Chapters 2 and 3 provide sufficient simulation evidence to show that our methods have an accuracy similar to other standard methods. But it would be interesting, in future research, to explicitly consider the consequences of Leeb and Pötscher (2005)'s findings for structural VAR analyses. 


\section{A Appendix}

\section{A.1 Additional Results for Christiano et al. (1999)}

Figure 4.10 plots the estimated impulse response of real GDP for different VAR models. It shows that the impulse response functions become more ragged the higher the number of lags that is included in the estimated VAR. The same observation can already be made for stationary autoregressive processes. Figure 4.11 shows the impulse response functions estimated by different AR models on a time series of length $T=160$ generated by the process

$$
y_{t}=0.4 y_{t-1}+0.2 y_{t-2}+0.2 y_{t-3}+u_{t},
$$

where $u_{t}$ are independent standard normally distributed errors. We see that the $\mathrm{AR}(9)$ implied impulse response function is more ragged than the $\mathrm{AR}(2)$ implied impulse response function in the following sense. Here we measure "raggedness" by the number of times the sign of the difference from the impulse response coefficient at horizon $h-1$ to horizon $h$ changes. For an $\mathrm{AR}(1)$ process with autoregressive coefficient $\alpha$ the impulse response function at horizon $h, \varphi_{h}$, is given by $\alpha^{h}$. So for $\alpha>1$ it is a monotonous function in $h$, and the number of the sign changes is hence 0 . For the impulse response function estimated by the $\operatorname{AR}(2)$, that is plotted in Figure 4.11, there are two changes in sign: $\varphi_{1}-\varphi_{0}$ is negative, $\varphi_{2}-\varphi_{1}$ is positive, and $\varphi_{3}-\varphi_{2}$ is negative again. In Table 4.6 the average number of sign changes for the impulse response functions estimated by AR models of different lag orders are given. For these results we simulated 10,000 time series of length $T=160$ of the process given above. We conclude that the increase in raggedness of the impulse response functions that follows from an increase in the estimating model's complexity is not an issue that only arises when treating systems of multiple time series, but one that is inherent in the modelling of univariate autoregressive time series already.

\begin{tabular}{r|ccccccccc} 
lags & 1 & 2 & 3 & 4 & 5 & 6 & 7 & 8 & 9 \\
\hline avg. changes & 0.0 & 1.3 & 2.1 & 2.7 & 3.8 & 4.9 & 6.2 & 7.1 & 7.8 \\
\hline
\end{tabular}

Table 4.6: The average number of sign changes in the differences of impulse responses from one horizon to the next. The number increases with the size of the AR model used for estimation.

Results for $\bar{p}=9$ For the results in the main text we have chosen $\bar{p}=11$. To show that the conclusions we drew from these are robust to the choice of $\bar{p}$ we give the results for $\bar{p}=9$ in this paragraph. Compared to the setting with $\bar{p}=11$ the FICirb and AIC-based responses are shifted downward for reserves and M1, and the peaks in the response of the commodity price index are flatter. 


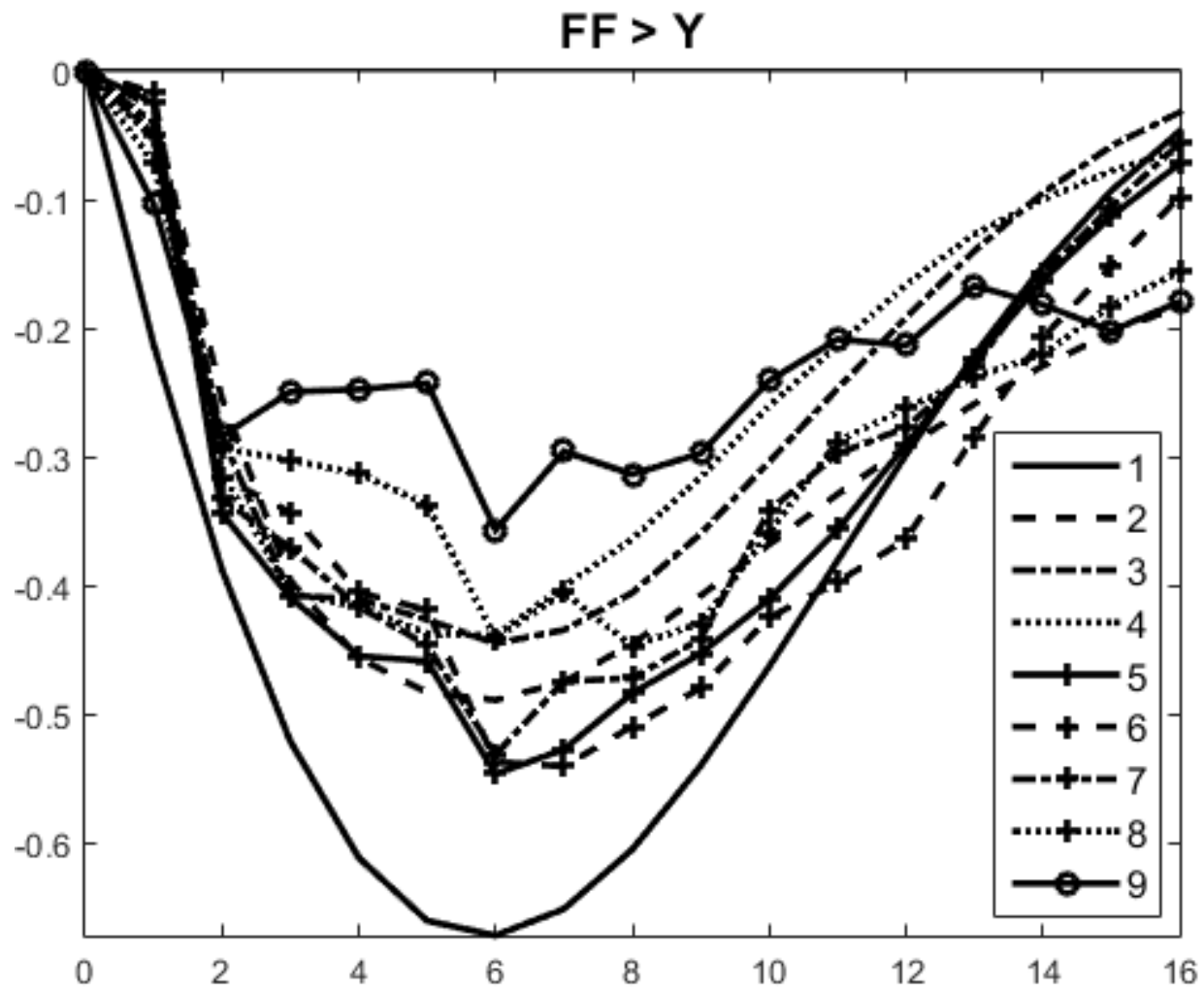

Figure 4.10: Christiano et al. (1999) data set IRs of the estimated $\operatorname{VAR}(i), i=1, \ldots, 9$. Estimated IR functions of higher order VARs are more ragged.

\section{A.2 Additional Results for Stock and Watson (2001)}

For the results of the main text we have chosen $\bar{p}=12$. To show that the conclusions we drew from these are robust to the choice of $\bar{p}$ we give the results for $\bar{p}=9$ in this paragraph. The point estimates in the following Figures $4.14-4.15$ should be compared to those in Figures 4.2 - 4.3 in the main text. The rough shapes of the estimated impulse responses functions are found to be similar. The responses of inflation and unemployment to a shock in inflation, for example, are at around 0.2 after 24 quarters for all of the different model criteria in Figure 4.2 as well as in Figure 4.14.

One effect of changing $\bar{p}$ can be observed in the responses implied by AIC model selection and FIC model averaging: While for $\bar{p}=12$ the responses that go lowest for an impulse in the federal funds rate are those implied by FIC averaging, for $\bar{p}=9$ the responses implied by AIC model selection reach the lowest points after such an impulse. This can be seen in the response of the federal funds rate in Figure 4.3, for example. There the only response that falls below $-0.2 \%$ for a 


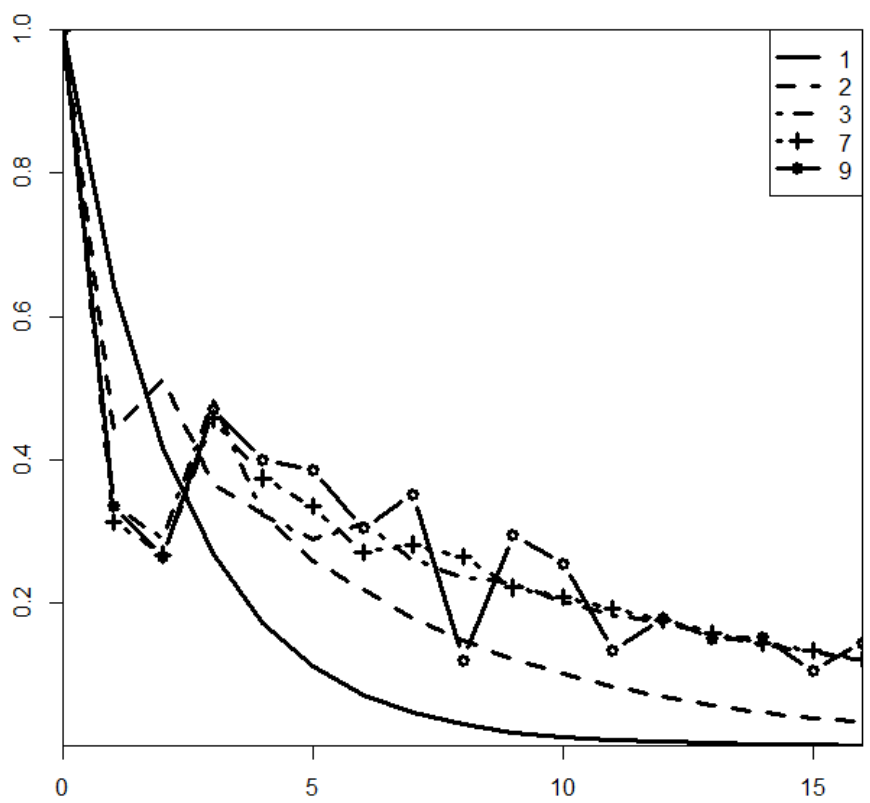

Figure 4.11: Estimated IRs for a stationary autoregressive process estimated with different AR models. Estimated IR functions of higher order AR's are more ragged.

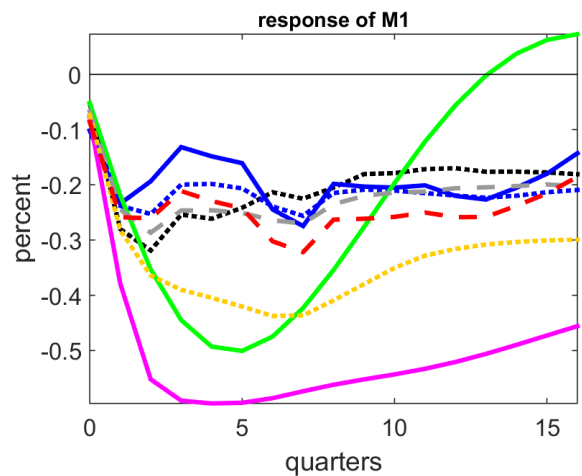

$\cdots \cdot \cdot$ VAR(4) - AIC $\cdots . \cdot$ sAICb - BIC - -EW - HQ $\cdots \cdots$ JMA - -FICirb

Figure 4.12: Christiano et al. (1999) data set IRs for $\bar{p}=9$. Criteria line colors: Black: VAR(4). Blue: AIC (solid), sAICb (broken). Green: BIC. Gray: Equal weights averaging. Magenta: Hannan-Quinn selection. Orange: Jackknife model averaging. Red: FIC averaging.

few periods, is that implied by FIC averaging, but it is the one implied by AIC selection in Figure 4.15. 

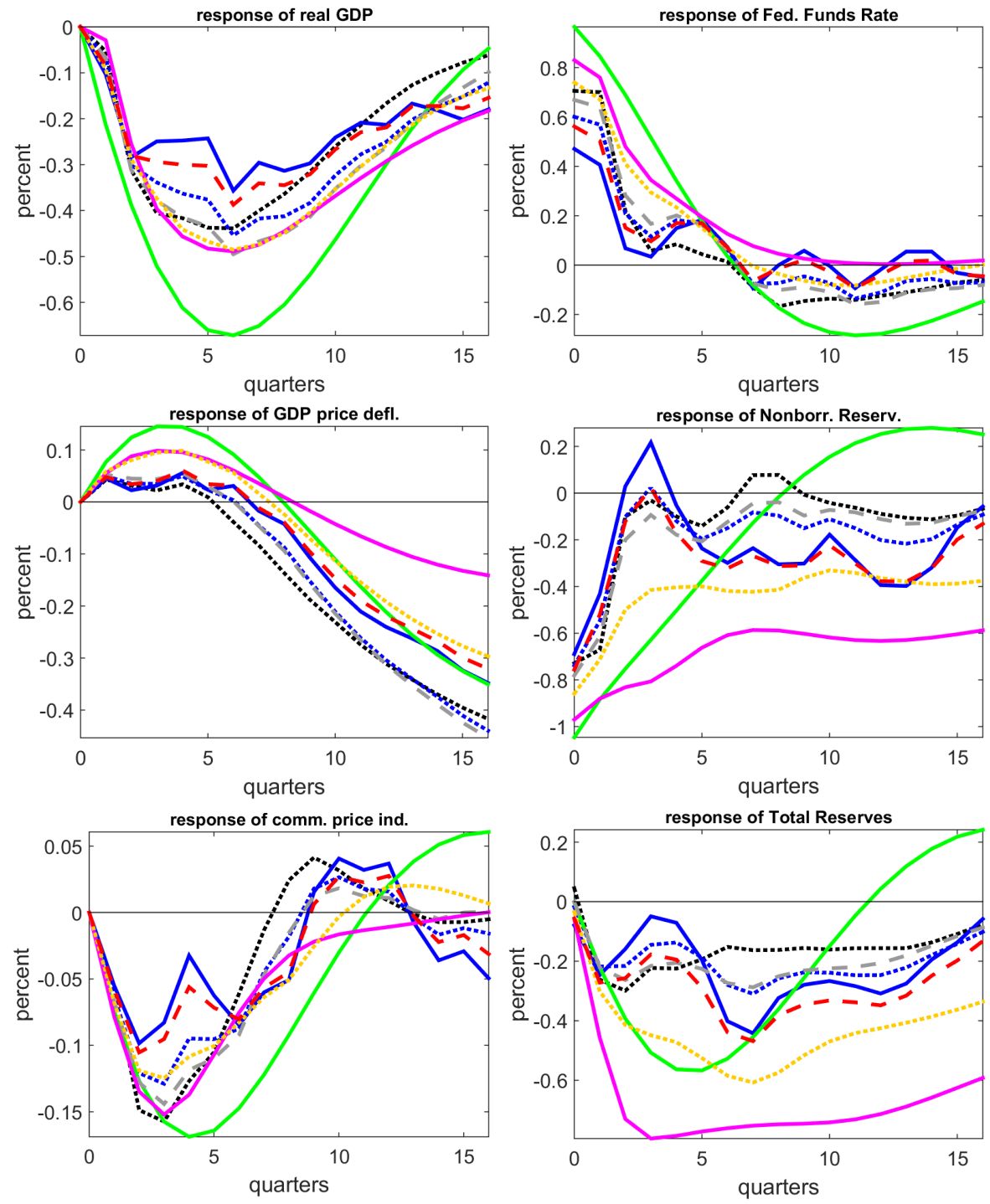

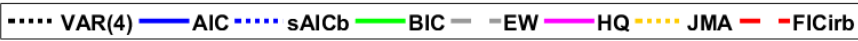

Figure 4.13: Christiano et al. (1999) data set IRs for $\bar{p}=9$. These are the counterparts to graphs in Figure 4.4 and following in the main text. Criteria line colors: Black: VAR(4). Blue: AIC (solid), sAICb (broken). Green: BIC. Gray: Equal weights averaging. Magenta: Hannan-Quinn selection. Orange: Jackknife model averaging. Red: FIC averaging. 

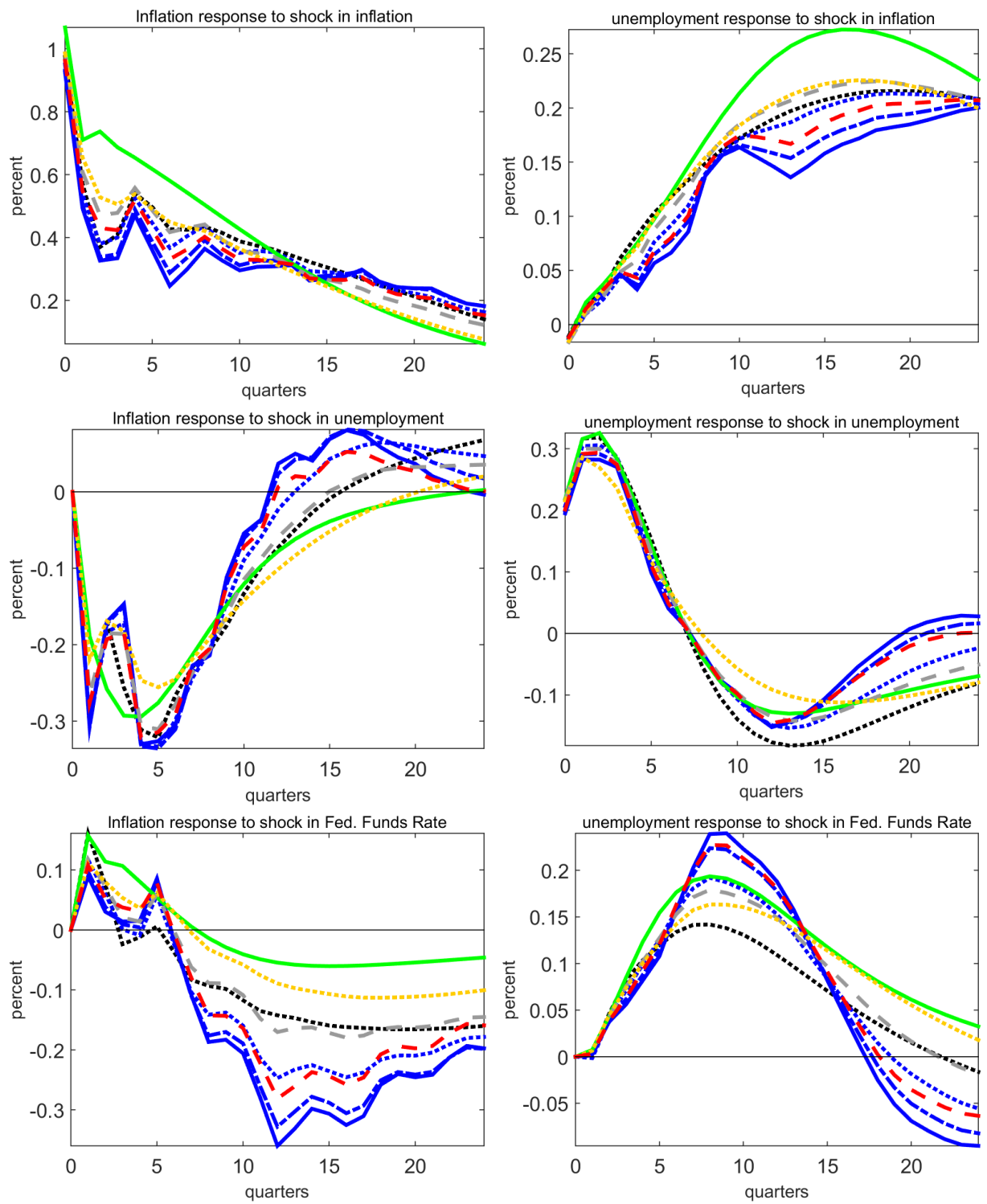

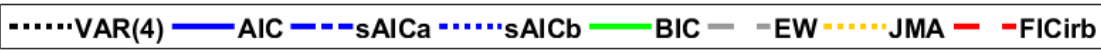

Figure 4.14: Stock and Watson (2001) data set IRs for $\bar{p}=9$.

These are the counterparts to graphs in Figure 4.2 and following in the main text. 

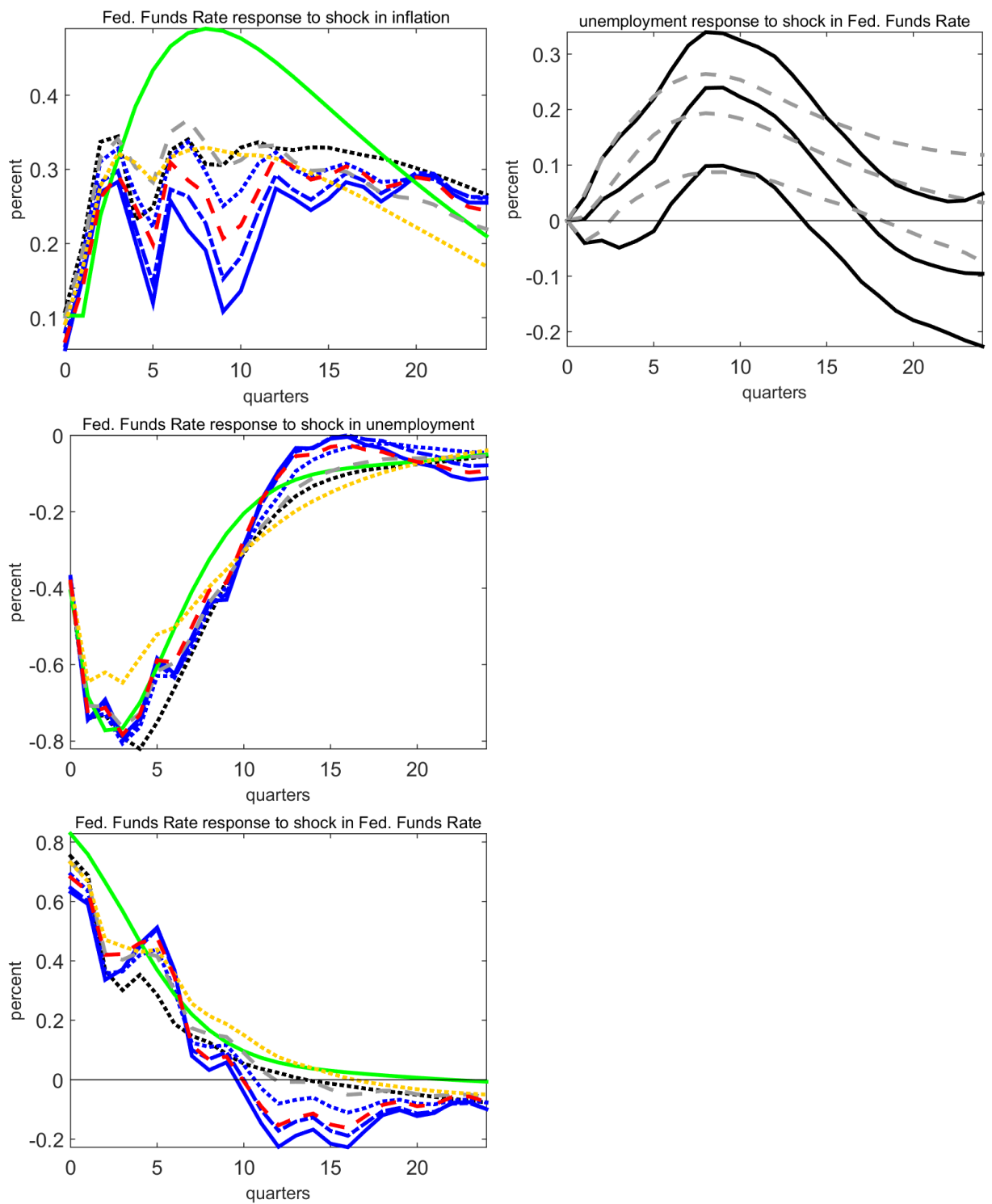

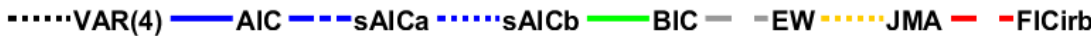

Figure 4.15: Stock and Watson (2001) data set IRs for $\bar{p}=9$.

Top right graph: Response of unemployment to a contractionary monetary policy shock: AIC (black) and BIC (gray) IR estimates with 66\% joint (adjusted Bonferroni) confidence bands. 


\section{Chapter 5}

\section{Conclusion}

In this thesis we extend the range of some model selection and model averaging criteria to multivariate stationary time series models by providing their theoretical properties, and we show their usefulness for the field of macroeconomic policy analysis.

Claeskens and Hjort (2003) enriched the concept of model selection by introducing the focused information criterion (FIC). The FIC, unlike other common information criteria that aim at a good average prediction quality, allows to identify those models that best estimate a focus parameter that can be defined by the user. The focus parameter is allowed to be any sufficiently regular function of the model parameters. Claeskens and Hjort (2003)'s theory makes use of a locally misspecified framework, where some of the model parameters shrink towards zero. We extend the theory about the FIC's asymptotic properties in regression settings as described by Liu (2015) to autoregressive models in Chapter 2. We offer a basis for defining stationary parameter regions for the locally misspecified framework and we discuss the role of the misspecification parameter, which cannot be estimated consistently. In Chapter 3 we then similarly extend the results about the asymptotic properties of the sAIC and sBIC averaging methods, that Zhang (2015) derived, to dynamic regression settings. That chapter also illustrates that a weight scaling parameter actually defines a whole set of smoothed estimators. We believe that smoothed averaging estimators could be defined for other model selection criteria as well, and that these would likewise have properties similar to their selection counterparts. This is a possible venue for future research. It would also be interesting to find out how the results of the series of papers by Leeb and Pötscher (2005) can be incorporated into our methodology. Together with the bootstrap procedure that we define for the smoothed averaging estimators the applied researcher now has on her hands another set of theoretically grounded model selection and averaging criteria, which we hope can be used beneficially on empirical data.

The motivations for the two sets of criteria, focused criteria, smoothed averages, stem from two different strategies of managing model uncertainty. The former is to acknowledge that finding the actual DGP is not as important as find- 


\section{CHAPTER 5}

ing the best predictor for the focus of the analysis. The latter is to acknowledge that combined estimates from multiple models may behave better than estimates from one single model. We show how these criteria can be applied to structural VAR analysis and offer a comparison with other commonly used model selection estimators in Chapter 4. We find that the sAIC estimator performs well compared to the AIC estimator, and that sampling data at a higher frequency leads to higher precision of the estimators. It is our hope that this application might influence other researchers to apply frequentist model averaging techniques in similar settings.

While we introduce new applications for existing model criteria we also have to note from our analyses that not one model criterion is best suited across all possible DGP's. So the evolution of econometrics will likely continue on a path where the suitability of different estimation methods is evaluated for each setting separately. It will remain an ongoing quest to evaluate existing criteria or find new ones that are best suited for a specific application. 


\section{Bibliography}

Abadir, K. M. and J. R. Magnus (2002). Notation in econometrics: A proposal for a standard. Econometrics Journal 5(1), 76-90.

Akaike, H. (1998). Information theory and an extension of the maximum likelihood principle. In Selected Papers of Hirotugu Akaike, pp. 199-213. Springer.

Anderson, T. W. (2003). An introduction to multivariate statistical analysis. Wiley-Interscience.

Bates, J. and C. Granger (1969). The combination of forecasts. Journal of the Operational Research Society 20(4), 451-468.

Belongia, M. T. and P. N. Ireland (2015). Interest rates and money in the measurement of monetary policy. Journal of Business 8 Economic Statistics 33(2), $255-269$.

Benkwitz, A., H. Lütkepohl, and M. Neumann (2000). Problems related to confidence intervals for impulse responses of autoregressive processes. Econometric Reviews 19(1), 69-103.

Berk, K. (1974). Consistent autoregressive spectral estimates. The Annals of Statistics 2(3), 489-502.

Bernanke, B. and A. S. Blinder (1992). The federal funds rate and the channels of monetary transmission. American Economic Review 82(4), 901-921.

Bernanke, B., M. Gertler, and M. Watson (1997). Systematic monetary policy and the effects of oil price shocks. Brookings Papers on Economic Activity 1997(1), 91-142.

Bernanke, B. and I. Mihov (1998). Measuring monetary policy. Quarterly Journal of Economics 113(3), 869-902.

Bose, A. (1988). Edgeworth correction by bootstrap in autoregressions. The Annals of Statistics 16(4), 1709-1722.

Buckland, S., K. Burnham, and N. Augustin (1997). Model selection: An integral part of inference. Biometrics 53(2), 603-618. 


\section{BIBLIOGRAPHY}

Burnham, K. P. and D. R. Anderson (2002). Model selection and multimodel inference: A practical information-theoretic approach. Springer.

Charkhi, A., G. Claeskens, and B. E. Hansen (2015). Minimum mean squared error model averaging in likelihood models. Working Paper.

Christiano, L. J., M. Eichenbaum, and C. L. Evans (1996). The effects of monetary policy shocks: Evidence from the flow of funds. Review of Economics and Statistics 78(1), 16-34.

Christiano, L. J., M. Eichenbaum, and C. L. Evans (1999). Monetary policy shocks: What have we learned and to what end? In Handbook of Macroeconomics, Volume 1, pp. 65-148.

Claeskens, G., C. Croux, and J. van Kerckhoven (2007). Prediction-focused model selection for autoregressive models. Australian 83 New Zealand Journal of Statistics 49(4), 359-379.

Claeskens, G. and N. L. Hjort (2003). The Focused Information Criterion. Journal of the American Statistical Association 98(464), pp. 900-916.

Claeskens, G. and N. L. Hjort (2008). Model Selection and Model Averaging. Cambridge University Press.

Davidson, J. (1994). Stochastic Limit Theory. Blackwell Publishing.

Davidson, J. (2004). Econometric Theory. Blackwell Publishing.

Davidson, R. and J. G. MacKinnon (1999). Econometric Theory and Methods. Oxford University Press.

de Pooter, M., F. Ravazzolo, and D. van Dijck (2007). Predicting the term structure of interest rates. Incorporating parameter uncertainty, model uncertainty and macroeconomic information. Tinbergen Institute Discussion Paper.

DiTraglia, F. (2016). Using invalid instruments on purpose: Focused moment selection and averaging for GMM. Journal of Econometrics 195(2), 187-208.

Eichenbaum, M. (1992). Comments on 'Interpereting the macroeconomic time series facts: The effects of monetary policy' by C. Sims. European Economic Review 36(5), 1001-1011.

Fujiwara, M. (1916). Ueber die obere Schranke des absoluten Betrages der Wurzeln einer algebraischen Gleichung. Tohoku Mathematica Journal 10.

Gani, A., A. V. Gribok, S. Rajaraman, W. K. Ward, and J. Reifman (2009). Predicting subcutaneous glucose concentration in humans: Data-driven glucose modeling. IEEE Transactions on Biomedical Engineering 56(2).

Hall, P. (1992). The Bootstrap and Edgeworth Expansion. Springer.

Hamilton, J. D. (1994). Time Series Analysis. Princeton University Press. 
Hannan, E. (1970). Multiple Time Series. John Wiley and Sons, Inc.

Hansen, B. E. (2005). Challenges for econometric model selection. Econometric Theory 21(1), 60-68.

Hansen, B. E. (2007). Least squares model averaging. Econometrica 75(4), 11751189.

Hansen, B. E. (2008). Least-squares forecast averaging. Journal of Econometrics 146(2), 342-350.

Hansen, B. E. (2016). Stein combination shrinkage for vector autoregressions. Working Paper.

Hansen, B. E. and J. S. Racine (2012). Jackknife model averaging. Journal of Econometrics 167(1), 38-46.

Hjort, N. L. and G. Claeskens (2003a). Frequentist model average estimators. Journal of the American Statistical Association 98(464), 879-899.

Hjort, N. L. and G. Claeskens (2003b). Rejoinder. Journal of the American Statistical Association 98(464), 938-945.

Imhof, J.-P. (1961). Computing the distribution of quadratic forms in normal variables. Biometrika 48(3/4), 419-426.

Ing, C.-K. and C.-Z. Wei (2005). Order selection for same-realization predictions in autoregressive processes. The Annals of Statistics 33(5), 2423-2474.

Ivanov, V. and L. Kilian (2005). A practitioner's guide to lag order selection for VAR impulse response analysis. Studies in Nonlinear Dynamics 83 Econometrics $9(1)$.

Jirak, M. (2012). Simultaneous confidence bands for Yule-Walker estimators and order selection. The Annals of Statistics 40(1), 494-528.

Johnson, N., S. Kotz, and N. Balakrishnan (1994). Continuous Univariate Distributions, Vol. 1-2. John Wiley \& Sons.

Juselius, K. (2006). The Cointegrated VAR Model. Oxford University Press.

Kilian, L. (1998a). Accounting for lag order uncertainty in autoregressions: The endogenous lag order bootstrap algorithm. Journal of Time Series Analysis 19(5), $531-548$.

Kilian, L. (1998b). Small-sample confidence intervals for impulse response functions. The Review of Economics and Statistics 80(2), 218-230.

Kilian, L. (2001). Impulse response analysis in vector autoregressions with unknown lag order. Journal of Forecasting 20(3), 161-179. 


\section{BIBLIOGRAPHY}

Kilian, L. (2013). Structural vector autoregressions. In N. Hashimzade and M. Thornton (Eds.), Handbook of Research Methods and Applications in Empirical Macroeconomics, pp. 515-554. Edward Elgar.

Kilian, L. and H. Lütkepohl (2017). Structural Vector Autoregressive Analysis. Cambridge University Press.

Kitagawa, T. and C. Muris (2016). Model averaging in semiparametric estimation of treatment effects. Journal of Econometrics 193(1), 271-289.

Leeb, H. and B. M. Pötscher (2005). Model selection and inference: Facts and fiction. Econometric Theory 21(1), 21-59.

Lewis, R. and G. C. Reinsel (1985). Prediction of multivariate time series by autoregressive model fitting. Journal of Multivariate Analysis 16(3), 393-411.

Liang, H., G. Zou, A. Wan, and X. Zhang (2011). Optimal weight choice for frequentist model average estimators. Journal of the American Statistical Association 106(495), 1053-1066.

Liu, C.-A. (2015). Distribution theory of the least squares averaging estimator. Journal of Econometrics 186(1), 142-159.

Liu, C.-A. and B.-S. Kuo (2016). Model averaging in predictive regressions. Econometrics Journal 19(2), 203-231.

Liu, Q. and R. Okui (2013). Heteroskedasticity-robust $C_{p}$ model averaging. 16(3), 463-472.

Lohmeyer, J., F. Palm, H. Reuvers, and J.-P. Urbain (2018). A Focused Information Criterion for locally misspecified vector autoregressive models. Econometric Reviews 0(0), 1-30.

Lütkepohl, H. (1990). Asymptotic distributions of impulse response functions and forecast error variance decompositions of vector autoregressive models. The Review of Economics and Statistics 72(1), 116-125.

Lütkepohl, H. (2005). New Introduction to Multiple Time Series Analysis. Springer.

Lütkepohl, H., A. Staszweska-Bystrova, and P. Winker (2015). Comparison of methods for constructing joint confidence bands for impulse response functions. International Journal of Forecasting 31(3), 782-798.

McManus, D. A. (1991). Who invented local power analysis? Econometric Theory 7(2), 265-268.

Paulsen, J. (1984). Order determination of multivariate autoregressive time series with unit root. Journal of Time Series Analysis 5(2), 115-127. 
Paulsen, J. and D. Tjøstheim (1985). On the estimation of residual variance and order in autoregressive time series. Journal of the Royal Statistical Society 47 (2), 216-228.

Pope, A. (1990). Biases of estimators in multivariate non-Gaussian autoregressions. Journal of Time Series Analysis 11(3), 249-58.

Quinn, B. G. (1980). Order determination for a multivariate autoregression. Journal of the Royal Statistical Society. Series B (Methodological) 42(2), 182-185.

Rohan, N. and T. V. Ramanathan (2011). Order selection in ARMA models using the Focused Information Criterion. Australian \& New Zealand Journal of Statistics 53(2), 217-231.

Schwarz, G. (1978). Estimating the dimension of a model. The Annals of Statistics $6(2), 461-464$.

Silvestrini, A. and D. Veredas (2008). Temporal aggregation of univariate and multivariate time series models: A survey. Journal of Economic Surveys 22(3), 458-497.

Sims, C. A. (1992). Interpreting the macroeconomic time series facts: The effects of monetary policy. European Economic Review 36(5), 976-1011.

Stock, J. H. and M. W. Watson (2001). Vector autoregressions. The Journal of Economic Perspectives 15(4), 101-115.

Stock, J. H. and M. W. Watson (2004). Combination forecasts of output growth in a seven-country data set. Journal of Forecasting 23(6), 405-430.

Uhlig, H. (2005). What are the effects of monetary policy on output? Results from an agnostic identification procedure. Journal of Monetary Economics 52(2), $381-419$.

Yuan, Z. and Y. Yang (2005). Combining linear regression models: When and how? Journal of the American Statistical Association 100(472), 1202-1214.

Zhang, X. (2015). Consistency of model averaging estimators. Economics Letters 130, 120-123.

Zhang, X., A. T. Wan, and G. Zou (2013). Model averaging by jackknife criterion in models with dependent data. Journal of Econometrics 174(2), 82-94. 


\section{Summary}

Econometricians are faced with the challenge of finding suitable models for the data to be analyzed. They face model uncertainty since the true data generating model is not known, and use model selection criteria or model averaging methods to deal with it. In this thesis we extend the range of applicability of some model selection and model averaging criteria to multivariate stationary time series models by providing their theoretical properties, and we show their usefulness for the field of macroeconomic policy analysis.

Claeskens and Hjort (2003) enriched the concept of model selection by introducing the focused information criterion. We extend the theory about its asymptotic properties in regression settings as described by Liu (2015) to autoregressive models in Chapter 2. We offer a basis for defining stationary parameter regions for the locally misspecified framework and we discuss the role of the misspecification parameter, which cannot be estimated consistently: The estimator of the misspecification parameter converges to a normally distributed random vector centered around the true misspecification parameter in the limit. This means that the estimate is still random in the limit, and the FIC expression does not converge in probability to the asymptotic MSE that it is intended to estimate. The chapter illustrates that point by including the infeasible estimator in the simulations, which corresponds to the FIC estimator, but with the estimate of the misspecification parameter replaced by the true misspecification parameter. The infeasible estimator is shown to have a lower MSE than the FIC estimators for large ranges of the simulations' parameter space. It is shown that the FIC estimator performs similarly to both AIC and BIC selection procedures. A plug-in average estimator (that also suffers from the fact that the misspecification parameter cannot be estimated consistently) is also discussed in that chapter.

In Chapter 3 we then similarly extend the results about the asymptotic properties of the smoothed AIC (sAIC) and smoothed BIC (sBIC) averaging methods, that Zhang (2015) derived, to dynamic regression settings. While for model selection based on the Akaike information criterion, the model with the lowest AIC score is selected, the smoothed AIC averaging estimator is defined as the weighted sum of the estimators from all models in the model set under consideration, with weights defined by the models' AIC scores. The chapter illustrates that the calculation of the weights as given by Zhang (2015) and in other publications, is actually only one representation of the set of smoothed AIC estimators, that is defined by a certain weight scaling parameter, cf. Section 3.3.1. We extend the 
results by Zhang (2015) for a time series setting by showing root-T consistency of the averaging estimators for specific ranges of the weight scaling parameter in Section 3.3, and propose a bootstrap method to estimate the distributions of the averaging estimators in Section 3.4. Using results of Bose (1988) we also show its asymptotic validity. In simulations we show the benefits of using sAIC-based instead of AIC-based estimators for estimating impulse response coefficients.

In Chapter 4 the model selection criteria and model averaging methods of Chapters 2 and 3 are applied in structural VAR analyses of empirical data. Structural VAR models are a central tool in macroeconomic and monetary policy analysis and we offer a comparison of the FIC and smoothed model averaging estimators with other commonly used model selection estimators in this setting. We find that AIC-based model averaging yields smoother and more precise estimates of impulse response functions than AIC-based model selection. In order to make our results more relevant we use the data from three different previous studies, Christiano et al. (1999), Stock and Watson (2001), and Uhlig (2005), which have been carefully selected for their similar analyses: They all estimate exogenous monetary shocks for US data sets of similar time periods, and apply the same structural parametrization for identifying impulse response functions. But the data sets do differ in sampling frequency and number of variables, and this allows us to compare the performance of the estimators across the different sampling frequencies of the data sets and to make suggestions on sampling frequency based on a comparison of estimator precision across the different data sets. We find that sampling data at a higher frequency (monthly) leads to higher precision than sampling at lower frequencies. At the same time, the results drawn from lower frequency (quarterly) data are more robust to the choice of model selection method. 


\section{Valorization Addendum}

This chapter discusses the societal value of the research presented in this book, what is innovative about the presented results, who can benefit from them, and why they are relevant.

Before treating such a special case, the societal value of one single book, it is an interesting exercise to reflect on how scientific and technological development in general has shaped our society. Are we smarter or better off than the people were 100 years ago? What are we better at now than we were back then? The answer may be obvious when we talk about inventions of physical apparatuses like the airplane, that was a whole new method of transportation, like information technology, one might imagine what a typical insurance company looked like and how many contracts per employee it was able to manage in the past and compare this to the high-tech offices of today, or like medical procedures, whose usefulness is also rather apparent. But as we consider shorter time periods, say the last 10 years, and scientific discoveries with less direct impact the question becomes more difficult to answer and raises follow up questions. For example: How can societal value and its progress even be measured?

One of many approaches, too many to name here, that is often taken to address this question is to measure economic growth. And it is a common understanding that the advancement of technology is a central component of economic growth. Developed countries usually see long periods of such growth, some of which should be due to scientific progress, even if indirectly. Now, economic growth is typically measured as the gross domestic product, GDP, of one or several nation(s). This is were this book is of value: All of the chapters, and especially Chapter 4 in a very illustrative manner, treat the type of linear models that are to date commonly used for analyzing the dynamics of economic variables like GDP and inflation. These are vector autoregressive models, VARs. VARs describe time series data for groups of variables which interact with each other. The term time series means that the values of the variables can be observed at consecutive time points within a certain time period. Chapter 3 shows that when estimating this kind of model the proposed model averaging procedures can deliver more exact estimates than the more commonly used, corresponding model selection procedures. Here the exactness, or precision of an estimator is measured by the mean squared error, MSE. So this dissertation provides methods that improve the measurement of some important macroeconomic variables This also means that the results can be used to measure the progress of one common indicator of societal value. Or said 
differently: One of the societal values of this book lies in being able to discuss societal value.

One group of users of VARs are central banks, which monitor the economy closely in order to find the level of interest rates that would lead to the inflation rate remaining in or returning to the mandated range. ${ }^{1}$ Even though the main instruments of a central bank are designed only to set the lending rates for banks, their decisions impact the whole economy as the effect of changes in these interest rates trickle down to the interest rates that companies and retail clients are offered. So, in the end, the spending behavior and savings rate of the population, and hence the inflation rate, are influenced by the central bank's decisions. This illustrates how important it is that central banks use good models. Naturally they employ a wide range of different models and well staffed research departments, but even if after publication of this thesis the discussed methods are not immediately picked up by the research staff of central banks, who often are well connected to academia and stay up to date with the latest research, the economic impact that econometric innovations have are potentially huge: Slight changes in estimation methods, if adopted in multiple central banks, could lead to immense financial effects in total.

But the fact that a central bank can directly set certain types of lending rates, but cannot directly influence inflation, means that the application of VAR models requires some further attention when specifying the concrete model: Econometricians use model selection criteria to decide whether to use a VAR model with shorter or longer memory (lower or higher lag order). Many of these model selection criteria aim at a model that explains the observed values best, but that is not necessarily the same model as the one that describes the reaction profiles of the variables in the system to a change in one of the variables best. These reaction profiles are called impulse response functions and they are used as the leading example of focus parameters ${ }^{2}$ throughout this book. Chapter 2 treats a model selection criterion that can be used to find the model that estimates these impulse response functions best. So, ultimately, the methods described in this book help central banks to better tune their monetary policy.

The model selection criterion called FIC - focused information criterion, that is being treated in Chapter 2, is not a new criterion. What is new about that chapter is that it extends the family of model types in which the FIC can be applied: The FIC was originally defined for usage with independent data, the type of data where one observation of the regressand is assumed not to be influenced by the other observations of the regressand. This book treats the case of time series data, where the value of a variable at one point in time does depend on the previous values. It is shown by means of mathematical analyses how the estimators of the impulse response functions, which are random variables, are distributed for a stable (in a certain sense defined in that chapter) VAR time series process that can be observed forever. Even though this can only be a thought experiment it is the cornerstone of the econometric science, where it goes under the name of

\footnotetext{
${ }^{1}$ Note that this is an exemplified depiction of the complex mission and range of instruments of most central banks.

${ }^{2}$ We use the term focus parameters, in analogy to the focused information criterion, for those parameters that are the main interest of the model's user.
} 


\section{VALORIZATION ADDENDUM}

asymptotic theory. The reason is that intuition gained from asymptotic theory often allows to draw conclusions for real world statistical applications with (only) a finite number of observations. The concept of consistency is an example for an asymptotic statement that is very important to econometricians. An estimator is named consistent if it delivers the true underlying parameter asymptotically. Some consistency results are also given and proven in Chapter 3 , for example. Additionally, it is common practice to amend scientific publications about asymptotic theory with simulation results, treating some prototypical cases of the type of data that would be observed in real life. This is also the case for Chapters 2 and 3 of this book. Via these two contributions, mathematical theory and finite sample intuition, statistical tools are considered ready for application. This is what this book offers, it makes the focused information criterion "ready to use" for time series data.

We now come back to the definition of the focused information criterion: It is defined such that the model, which has the highest estimated precision, is selected based on the asymptotic results. The precision in question is that of estimating a certain focus parameter chosen by the user of the model. The leading example are impulse response functions, as mentioned above, but many other definitions of focus parameters are also covered by the results of this book: Any focus parameter is allowed that can be written as a smooth (continuously differentiable) function of the model's basic coefficients. This is especially interesting in VAR models since the number of basic coefficients is determined by the product of the number of variables one wants to consider with the length of the memory assumed by the model, a number that becomes relatively large even for low dimensional systems. It is hence sensible to expect that not all of the basic coefficients are equally interesting to the user of the model and by using the FIC the model (specification and) selection process can be geared towards those coefficients that are considered important.

One might wonder why there are different model selection criteria and why one would want to direct the selection process towards only some of the model coefficients and not all of them. Why do econometricians not simply find the correct model that fits the observed data perfectly? That is because econometrics is not a science of certainties. Instead it is a field of research that fine tunes its methods and makes them available for wider ranges of model settings. But in the end any model will just be an approximation, an attempt to explain some random outcomes. We see this from the discussion above, that explains that only if one was able to observe the outcomes of one process forever would it be possible to draw definite conclusions about its properties. And this would even only be the case if all the relevant variables were included in the model. That is why different researchers might use different models and methods for the same data. Some methods will explain one characteristic of the data better while others will explain other characteristics better. The model class, model specification, and model selection criterion a researcher applies to a set of data represent different ideas about the possible data generation process. It is one purpose of econometric literature to serve as a library of these different ideas. As discussed above, new ideas for approaching different kinds of data are made 
"ready to use" by deriving their mathematical properties or the properties of existing methods are described for new kinds of processes. So even after decades of statistical research the literature is still growing as new and refined methods are designed inspired by users of random data who wonder how one or another procedure would perform for their application. This is another contribution of this book: It adds a piece to the library of mathematical derivations, and guidelines for the application of the presented methods. In Chapter 2, for example, the role of the locally misspecified parameters is discussed and it is shown that the problem of not being able to estimate them consistently leads to a worse performance of the FIC estimator than would be the case if the parameters were known. Other researchers can build on these derivations and do not need to formulate them themselves anymore in cases where they want to apply the FIC to time series data, for example.

Since all models can only be approximations, users of statistical methods do not have to confine themselves to the process of model selection, which is the process of finding the best suited specification for the data at hand from a predefined range of models. Model averaging is an alternative to model selection. In model averaging, instead of calculating scores for all the models under consideration, and subsequently choosing the one with the best (lowest) score for further use, weights are calculated for all the models. These are then used for calculating an averaged estimate as the weighted average of the estimates from all considered models. It turns out that such averaged estimators can be more precise than estimators based on single models. This is shown in Chapter 3. Like Chapter 2, Chapter 3 provides the asymptotic theory of methods that had already been defined for independent data settings, for the time series setting. The methods are smoothed AIC and smoothed BIC averaging. For those economists who want to use the idea of model averaging on a focus parameter that they are interested in in a time series setting, Chapter 2 shows how such a FIC averaging estimate can be meaningfully defined.

The theoretical results alluded to above lead to point estimates. As an example, an estimation procedure with a VAR model describing an economy could lead to the estimated result that if the central bank's monetary policy variable increased by one standard deviation from one quarter to the next unexpectedly, then the effect of this change on the gross domestic product two quarters later will be a reduction by $0.3 \%$. But this number alone, this so called point estimate, does not give any information on the degree of certainty that the model attaches to this estimate. Such is usually represented by confidence intervals. In this case a $95 \%$ confidence interval might for example be described by the starting and end points $-0.35 \%$ and $-0.25 \%$, meaning that with $95 \%$ probability the reduction in GDP will be in this range. Wider confidence intervals represent greater uncertainty. Confidence bands are the analog to confidence intervals for functions, and can be obtained by joining neighboring confidence intervals together. One common presentation of confidence bands is for temperature forecasts, where it can typically be observed that the confidence bands widen for longer prediction horizons as the precision of the estimates decreases. Chapter 2 of this book also presents a method of calculating confidence bands around the estimates from FIC model selection and FIC model averaging estimates. This method relies on the asymptotic distribution 


\section{VALORIZATION ADDENDUM}

of the estimators, which can be more or less relevant for finite sample results. But the estimators of Chapter 3 are also presented together with methods for constructing confidence bands. These, however, are based on the finite sample distribution of the estimators as they rely on so called bootstrapping methods. This means that the methods are relevant for all sample sizes, and hence very valuable for practitioners. Since the AIC is a heavily used model selection criterion, the smoothed AIC estimator together with the possibility of calculating confidence bands as presented in Chapter 3 should find many users among users of time series data.

While Chapters 2 and 3 provide the mathematical framework for some new econometric tools and simulation studies to illustrate their properties, Chapter 4 applies the aforementioned model selection and model averaging criteria and the bootstrap algorithm for constructing confidence bands to empirical data. The data is the same as has been used in previous econometric publications that analyse the effects of monetary policy on US macroeconomic variables by modelling VAR processes. These publications are well known among practitioners. Nowadays there are plenty of model selection criteria available whose theoretical properties are described in the literature, but it may not always be obvious how much the choice of model selection criterion affects estimation results. That is why the application of different model selection and model averaging criteria to these well known data sets helps practitioners to gain intuition on how sensible the results one might typically expect from monetary VAR models are to the choice of model selection criterion. Arguing from a slightly different perspective: There are many different methods and mathematical ideas that a researcher could spend a lot of time and paper on to write down the corresponding asymptotic theory only with the goal of producing material to impress publishers or employers. After all, the econometric literature mostly consists of methods that work, that have some use. So there would potentially still be a lot to be written down on methods that are of limited use only. But by comparing our methods with previous studies in empirical research and showing that our methods deliver estimates in similar ranges as existing, heavily used criteria, we show that our methods are not completely without application in empirically relevant cases.

To end this valorization section note that the vector autoregressive - VAR models for which the methods in this book are discussed have autoregressive - AR - models as a special case. These have applications in many different fields, for example climate research, finance, physics, medicin, cf. e.g. Gani et al. (2009). And lastly, all the discussed methods can easily be implemented with standard statistical software packages like $\mathrm{R}$ and Matlab. All the necessary formulas and definitions are written down in this text. 


\section{Curriculum Vitae}

Jan Lohmeyer was born on September 12, 1980 in Sulingen, Germany. In 2001 he started studying Mathematics with a minor in Economics at the Universität Bielefeld in Germany, where he graduated as a Diplom-Wirtschaftsmathematiker in 2007. From 2011 until 2013 Jan studied at Københavns Universitet, Denmark, graduating as a MSc in Economics (finance). Also in 2013 Jan was hired as a PhD candidate at the Department of Quantitative Economics of Maastricht University, funded by an NWO project on model uncertainty, under the supervision of Prof. dr. Franz Palm and Prof. dr. Jean-Pierre Urbain. The results of his research are presented in this dissertation, one chapter of which has been published in an international, refereed academic journal. Jan has presented his research at several international conferences and workshops.

From 2007 until 2011 Jan was an actuarial analyst at Zurich Financial Services, based in Bonn. Since 2016 he has been working as a market risk controller at the Düsseldorfer Hypothekenbank AG. 